\title{
Synchronisation interagierender komplexer Systeme
}

\author{
Dissertation \\ zur Erlangung des Doktorgrades \\ der Mathematisch-Naturwissenschaftlichen Fakultäten \\ der Georg-August-Universität zu Göttingen
}

\author{
vorgelegt von \\ Lutz Junge \\ aus \\ Einbeck
}

Göttingen 2000 
D7

Referent: PD Dr. Ulrich Parlitz

Korreferent: Prof. Dr. Theo Geisel

Tag der mündlichen Prüfung: 22. Juni 2000 


\section{Kurzzusammenfassung}

Die vorliegende Arbeit beschäftigt sich mit der Synchronisation chaotischer dynamischer Systeme. Synchronisationsphänomene periodischer Oszillatoren sind wohlbekannt und gut verstanden. Aber auch chaotische Systeme können miteinander synchronisieren, wobei eine Vielzahl neuer Phänomene auftritt.

Bei identischen Systemen kann bei entsprechender Kopplung identische Synchronisation auftreten, wo die Systeme asymptotisch die exakt gleiche Zeitentwicklung besitzen. Zu diesem Zweck steht eine Vielzahl von Kopplungstechniken zur Verfügung, die aber bisher nicht die (chaotische) Eigendynamik der Systeme ausnutzen. Aus diesem Grund führen wir eine dynamische Kopplungsstrategie ein, welche von der Systemdynamik definiert wird und stabile Synchronisation garantiert.

Synchronisation wird nicht nur bei niederdimensionalen Systemen beobachtet, sondern tritt auch bei hochdimensionalen und räumlich ausgedehnten Systemen, beschrieben durch partielle Differentialgleichungen, auf. Die existierenden Kopplungsstrategien niederdimensionaler Systeme lassen sich aber nicht trivial auf Systeme mit ausgedehntem Raum verallgemeinern, so daß eine Kopplung, basierend auf räumlich ausgedehnten Meßsensoren bzw. Aktuatoren, vorgeschlagen wird, die auch in Experimenten anwendbar ist. Bei nichtidentischen Systemen treten kompliziertere Synchronisationsarten auf. Die stärkste Form ist verallgemeinerte Synchronisation, bei der die Flüsse in einem funktionalen Zusammenhang stehen. Anhand von mehreren Beispielen wird gezeigt, daß i.A., die Funktion implizit definiert werden muß und auch fraktal sein kann.

Eine schwächere Form ist die Phasen- bzw. Frequenzsynchronisation, die nur partielle Gemeinsamkeiten der Flüsse fordert, so daß eine feste Beziehung zwischen den Phasen (falls eine geeignete Phasenvariable eingeführt werden kann) der Systeme besteht, während die Amplituden völlig unkorreliert bleiben können. Diese Variante konnten wir auch bei Paaren gekoppelter partieller Differentialgleichungen direkt nachweisen. Für stärkere Kopplung der Systeme haben wir darüberhinaus starke Anzeichen für das Auftreten von verallgemeinerter Synchronisation gefunden.

Die vorgestellten Kopplungsstrategien werden schließlich in der Parameter- und Zustandsrekonstruktion von hyperchaotischen und partiellen Differentialgleichungen angewendet, wobei besonderer Augenmerk auf den Einfluß von verrauschten Meßsignalen gelegt wird. 


\section{Inhaltsverzeichnis}

1. Einleitung 1

1.1. Rhythmen . . . . . . . . . . . . . . . . . . 1

1.2. Aperiodische Dynamik . . . . . . . . . . . . . . 2

1.3. Konventionen . . . . . . . . . . . . . . . . . 5

2. Varianten der Synchronisation gekoppelter (chaot.) dynamischer Systeme $\mathbf{6}$

2.1. Synchronisation identischer Systeme . . . . . . . . . . . . . 7

2.1.1. Der Übergang . . . . . . . . . . . . . . . . . . . . . . . . . . . 12

2.1.2. Einfluß von Rauschen . . . . . . . . . . . . . . . . . . . . . 15

2.2. Verallgemeinerte Synchronisation . . . . . . . . . . . . 16

2.2.1. Nachweis . . . . . . . . . . . . . . . . . . . 17

2.2.2. Eindeutigkeit der Abbildung . . . . . . . . . . . . . . . 19

2.2.3. Glattheit der Abbildung . . . . . . . . . . . . . . . . . 24

2.3. Frequenz- und Phasensynchronisation . . . . . . . . . . . 27

3. Die treibende Kraft $\quad \mathbf{3 0}$

3.1. Wahl der Kopplung . . . . . . . . . . . . . . . . . . . . . . 31

3.1.1. Stabile Subsysteme . . . . . . . . . . . . . . . . 31

3.1.2. Einfügen von Dissipation . . . . . . . . . . . . . . 31

3.1.3. Aktiv-Passiv Zerlegung . . . . . . . . . . . . . . 32

3.1.4. Diskret in der Zeit . . . . . . . . . . . . . . . . . . 35

3.1.5. Der nichtlineare Beobachter . . . . . . . . . . . . . . . 36

3.2. Direkte Kompensation des Schmetterlingseffektes . . . . . . . . . . . . 37

3.2.1. Dynamische Kopplung im Phasenraum . . . . . . . . . . . . . . . 38

3.2.2. Lokale Maße der Instabilität von Systemzuständen . . . . . . . . . 41

3.2.3. Anwendung am Hénonsystem . . . . . . . . . . . . . . . . . . 45

3.2.4. Partitionierung des Phasenraumes . . . . . . . . . . . . 56

3.2.5. Dynamische Kopplung bei ODEs. . . . . . . . . . . . . . . . . . 63

3.2.6. Dynamische Kopplung bei höherdimensionalen Systemen . . . . . 67

3.2.7. Informationstheoretische Aspekte . . . . . . . . . . . 70

3.2.8. Diskussion und Ausblick . . . . . . . . . . . . . . . . 72

4. Synchronisation und Kontrolle räumlich ausgedehnter Systeme $\mathbf{7 5}$

4.1. Felder gekoppelter niederdimensionale Systeme . . . . . . . . . . . . . 76

4.2. Partielle Differentialgleichungen . . . . . . . . . . . . . . . . . 79 


\section{Inhaltsverzeichnis}

4.3. Synchronisation mit Sensoren . . . . . . . . . . . . . . . . . 81

4.3.1. Kuramoto-Sivashinski Gleichung . . . . . . . . . . . . . . 82

4.3.1.1. Optimale Position der Sensoren . . . . . . . . . . . . 89

4.3.1.2. Unterdrückung von raumzeitlichem Chaos mittels Sensorkopplung . . . . . . . . . . . . . 9 90

4.3.2. Intensive Untersuchung der Eigenschaften der Sensorkopplung am Beispiel der Ginzburg-Landau Gleichung . . . . . . . . . . . . . . 91

4.3.2.1. Gegenseitige Abhängigkeiten der Kopplungsparameter bei der Sensorkopplung . . . . . . . . . . . . . 94

4.3.2.2. Stabilisierung des homogenen Grundzustandes $u \equiv 0$. . 98

4.3.2.3. Synchronisation und Kontrolle von raumzeitlichem Chaos in lokalen Regionen . . . . . . . . . . . . . . . . . 100

4.3.2.4. Ein paar abschließende Worte zur Sensorkopplung . . . . 101

4.4. Frequenz- und Phasensynchronisation von räumlich ausgedehnter Systeme 102 4.4.1. Einseitig gekoppelte Ginzburg-Landau Gleichungen . . . . . . . . . 102 4.4.2. Beidseitig gekoppelte Ginzburg-Landau Gleichungen . . . . . . . . 108

5. Anwendung der chaotischen Synchronisation in der Systemidentifikation $\mathbf{1 1 2}$

5.1. Synchronisationsbasierende Bestimmung von Systemparametern . . . . . . 114

5.2. Hyperchaotische verrauschte Systeme . . . . . . . . . . . . . . . . 116

5.3. Räumlich ausgedehnte Systeme . . . . . . . . . . . . . . . . . . 121

5.3.1. Kuramoto-Sivashinski Gleichung . . . . . . . . . . . . . . 122

5.3.2. Ginzburg-Landau Gleichung . . . . . . . . . . . . . . . . . . . . 124

$\begin{array}{ll}\text { 6. Zusammenfassung und Ausblick } & 126\end{array}$

$\begin{array}{ll}\text { A. Ableitung der lokal dynamischen Kopplung bei ODEs } & 128\end{array}$

$\begin{array}{ll}\text { Literaturverzeichnis } & 130\end{array}$ 


\section{Einleitung}

\subsection{Rhythmen}

Die Geschichte der Synchronisation gekoppelter Systeme reicht bis ins 17. Jahrhundert zurück, als der niederländische Physiker Christian Huygens zwei an einem Holzbalken aufgehängte Pendeluhren beobachtete [48]. Die beiden Pendel bewegten sich stets in entgegengesetzter Richtung (Phasenunterschied von $\pi$ ) und nach einer Störung der Bewegung kehrten sie wieder rasch in diesen Zustand zurück. Huygens fand die Ursache in der Kopplung durch den Balken, den die Pendel in unmerkliche Schwingungen versetzten und der praktisch identischen Bauart der Uhren, welche auf identische Eigenfrequenzen führt. Anfang des letzten Jahrhunderts wurde die Problematik der Synchronisation von periodischen Systemen von van der Pol wieder aufgegriffen. In [133] wurde gezeigt, daß die Frequenz eines Triodengenerators unter schwachem harmonischen Antrieb mitgeführt wird, so daß dieser in Frequenz und Phase mit dem in der Frequenz leicht verstimmten Antriebssignal synchronisiert. Diese und Folgearbeiten hatten großen Einfluß auf das sich zu dieser Zeit rasch entwickelnde Feld der Radiokommunikation, dessen Grundelemente solche Generatoren waren. Die Synchronisation von periodischen Oszillatoren ist aus der heutigen Gesellschaft, obwohl meist unbemerkt, nicht mehr wegzudenken. So müssen bei jedem Kommunikationssystem Sender und Empfänger in Frequenz und Phase miteinander synchronisiert sein (welches heute durch sogenannte „phase locked loops“(PLLs) erreicht wird), bei Monitoren und Fernsehern muß der Elektronenstrahl auf die horizontalen und vertikalen Bildwiederholfrequenzen abgestimmt werden, im Verkehr werden Ampelphasen mit der mittleren Geschwindigkeit des Verkehrsflusses synchronisiert, etc. Neben technischen Systemen ist die Angleichung von Rhythmen in der Natur, verursacht durch eine Verbindung verschiedener Einzelsysteme, wesentlich für das Verständnis und Funktionieren komplexer Systeme, wie z.B. Zellverbände, Evolutionsdynamik und auch der Selbstorganisation solch gearteter Verbände. Ein entscheidender Punkt zum Verständnis der Synchronisation und auch der Selbstorganisation ist die Möglichkeit der Systeme zum Interagieren, welches in der Technik durch äußere Einflußnahme realisiert wird. In natürlichen Systeme hingegen ist die Kopplung oft schon intern vorhanden, z.B. in Form eines Wettbewerbes um gemeinsam genutzte Ressourcen. In Ökosystemen können dadurch z.B. neben den natürlichen auch neue Rhythmen entstehen [10], welche bei isolierten Spezies nicht auftreten. Ein weiteres interessantes Beispiel sind in Südamerika lebende Glühwürmchen, welche sich bevorzugt in Schwärmen auf Bäumen aufhalten. Die männlichen Glühwürmchen wollen dabei mittels kurzer Lichtblitze Weibchen zur Paarung anlocken. Das kollektive „Feuern“ von kurzen Lichtblitzen sollte dann zu 


\section{Einleitung}

einem stochastisch flackernden Leuchten der Schwärme führen, stattdessen wird aber ein zeitlich synchronisiertes großfächiges Blitzen beobachtet. Der Schwarm koppelt damit auf die einzelnen Glühwürmchen zurück, welches zu synchronisierter Lichtausstrahlung führt [14, 34]. Dies ist ein schönes Beispiel dafür, daß die Kopplung von vielen (leicht) komplexen Systemen nicht unbedingt zu einer hochkomplexen Dynamik führen muß. Stattdessen führt die Interaktion bei (ähnlichen) Systemen oft zu einer starker Regularisierung des kollektiven Verhaltens, was allgemein etwas vage unter dem Oberbegriff Selbstorganisation zusammengefaßt wird. Daß solch ein Verhalten nicht unbedingt stabil sein muß, zeigt eine Studie von der Synchronisation beim Applaudieren [77], wie sie z.B. bei Konzerten oder im Theater beobachtet wird. Dabei wurde festgestellt, daß sich die größte Lautstärke durch zeitlich unsynchronisiertes schnelles Klatschen erzielen läßt. Jeder Zuhörer hat seine eigene Applaudierperiode, welche über das gesamte Publikum zu einer Verteilung der Klatschfrequenzen führt. Es wird mit einem langsamen Klatschen langer Periode begonnen, wobei die Zuhörer schnell ihren Rhythmus mit dem gesamten Auditorium synchronisieren, welches zu einem periodischen Signal führt, wodurch aber die Laustärke auch etwas abnimmt. Da größere Zustimmung wohl mit höherer Lautstärke gleichgesetzt wird, fangen einige Personen an mit doppelter Frequenz zu applaudieren, die anderen Folgen und das periodische Klatschen ist einem unsynchronisiertem aber lauteren Applaus gewichen. Aufgrund der in diesem Regime breiten Verteilung der individuellen Klatschfrequenzen ist nun keine Synchronisation mehr möglich. Wenn der Applaus wieder etwas abflaut, gewinnt der langsame Rhythmus wieder an Gewicht und synchronisierter Applaus setzt wieder ein, welches sich einige Male fortsetzen kann. Vom physikalischen Standpunkt aus ist das Publikum ein selbstorganisiertes bistabiles System, welches periodisch zwischen zwei instabilen Gleichgewichtslagen oszilliert. Der innere Wunsch eines einheitlichen periodischen Rhythmus konkurriert dabei mit dem Bestreben nach hoher Lautstärke (Zustimmung).

\subsection{Aperiodische Dynamik}

Die bis hier betrachteten Systeme zeigen von sich aus sehr reguläre oder sogar periodische Eigendynamik, so daß Phänomene wie Frequenzmitnahme oder das Einstellen einer festen Phasendifferenz auch bei leicht unterschiedlichen Systemen intuitiv einsichtig und auch theoretisch gut verstanden sind. Aber was bedeutet Synchronisation bei komplexeren chaotischen Systemen, mit ihrer oft fast erratisch anmutenden Dynamik?

Dazu betrachten wir zuerst direkt das aus den griechischen Vokabeln syn (gemeinsam, gleich) und chronos (Zeit) zusammengesetzte Wort Synchronisation, welches frei vielleicht als den gleichen Rhythmus zur gleichen Zeit besitzend übersetzt werden könnte, was den Zustand periodischer gekoppelter Systeme sehr treffend beschreibt. Eine Interpretation für beliebige Systeme wurde in [17] vorgeschlagen, wo auf sehr allgemeinener Ebene Synchronisation als eine gemeinsame Eigenschaft zur gleichen Zeit besitzend definiert wird. Diese Eigenschaft muß sauber definiert werden und auch eindeutig meßbar sein. Weiterhin wird in [17] eine Synchronisationsnorm gefordert, die ein Kriterium für das Auftreten von Synchronisation bezüglich dieser Eigenschaft liefert. Diese Metadefini- 


\section{Einleitung}

tion ist motiviert durch die im letzten Jahrzehnt ständig wachsende Anzahl chaotischer Synchronisationsarten (siehe auch Kap. 2) und versucht, diese zu formalisieren und unter einem gemeinsamen (höheren) Kontext wieder zusammenzufassen. Die obigen Beispiele periodischer Synchronisation würde man in diesem Kontext Synchronisation bezüglich der Phase bzw. Frequenz bezeichnen, welche auch bei vielen chaotischen Systemen beobachtet wird (siehe Abschn. 2.3). Die vielleicht bekannteste Variante von chaotischer Synchronisation ist die identische Synchronisation (IS), welche auf den Zustand des treibenden Systems bezogen ist und bei Systemen von der exakt gleichen Art auftritt (siehe Abschn. 2.1). Bei IS gleichen sich die Zustände der gekoppelten Systeme asymptotisch an, so daß sie nach einer transienten Phase ununterscheidbar sind. Diese Variante wurde zuerst 1983 von Fujisaka \& Yamada [32] eingeführt und numerisch nachgewiesen. Die chaotische Synchronisation wurde inzwischen aber auch in vielen anderen naturwissenschaftlichen Gebieten nachgewiesen. So wurde Synchronisation von Chaos bei zwei einzelnen gekoppelten Neuronen [30] experimentell beobachtet, welches die Bedeutung der Synchronisation bei der Informationsverarbeitung neuronaler Systeme, wie z.B. dem Gehirn [31, 36, 119, 123] untermauert, in der Optik bei Lasersystemen [113, 129, 138], in der Festkörperphysik an stromkontrollierten Systemen von Josephson-Kontakten [23], beim resonanten Tunneln in Halbleitern [140] und in MEG-Messungen am Gehirn [131], dem Herzschlag mit dem Atmen [118], um nur einige der wichtigsten Beispiele zu nennen. Dies unterstreicht die stürmische Entwicklung dieses Teilaspektes der nichtlinearen Dynamik, wobei die Bedeutung und Anwendung inzwischen weit über die nichtlineare Dynamik hinausgeht. Abbildung 1.1 zeigt die Anzahl der referierten Veröffentlichungen zur Synchronisation von Chaos in Abhängigkeit des Erscheinungsjahres, wie sie eine Recherche in der Verbunddatenbank INSPEC ergab. Die Meilensteine sind 1983 die erste direkte Veröffentlichung zur chaotischen Synchronisation von Fujisaka \& Yamada [32] und vor allem der 1990 erschienene Artikel von Pecora \& Carroll [99], mit der vorgeschlagenen potentiellen Anwendung der chaotischen Synchronisation zur sicheren Nachrichtenübertragung. Diese Arbeit sorgte praktisch für eine Inertialzündung auf diesem Gebiet, welche eine exponentiell ansteigende Flut von Veröffentlichungen nach sich zog (siehe Abb. 1.1), wobei der Hauptantrieb sich schnell von der sicheren Nachrichtenübertragung zu einem breiten Anwendungsspektrum (siehe obige Beispiele) hin wandelte. Hierbei ist noch zu bemerken, daß sich die Neurophysik schon länger und unabhängig von dieser Entwicklung für Synchronisationseffekte, wie sie z.B. im Cortex auftreten, interessiert [31, 36, 123]. In dieser Arbeit wird versucht, einen Überblick über das ganze Spektrum der chaotischen Synchronisation zu geben, wobei wir uns auf dissipative dynamische Systeme beschränken. Aufgrund der Natur dieser Arbeit werden in den einzelnen Kapiteln die eigenen Arbeiten ausführlicher diskutiert, wobei aber viel Wert auf die Einordnung unserer Arbeit in den allgemeinen Kontext gelegt wurde. Die vielfältigen Formen der chaotischen Synchronisation werden in Kap. 2 vorgestellt und deren Zusammenhänge diskutiert. Die wichtigsten Arten der Kopplung dynamischer Systeme mit Anwendung bei der chaotischen Synchronisation werden im ersten Teil von Kap. 3 eingeführt und in Kap. 4 auf räumlich ausgedehnte Systeme verallgemeinert. Zusätzlich wird eine neue Kopplungsstrategie vorgeschlagen, die für ein gegebenes System identische Synchronisation garantiert und die Systemdynamik zur Kopplung ausnutzt. Das Auftreten von 


\section{Einleitung}

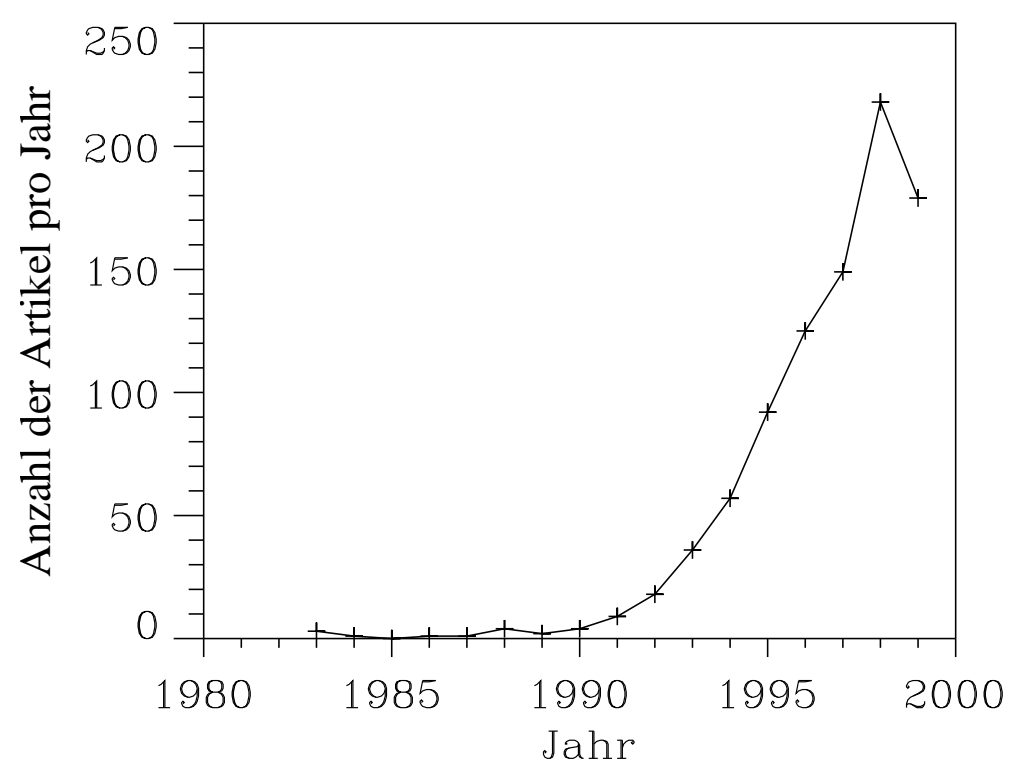

Abbildung 1.1.: Entwicklung der Anzahl der referierten Veröffentlichungen zur chaotischen Synchronisation in Abhängigkeit des Erscheinungsjahres (Quelle INSPEC). Dies dürfte die untere Grenze der Anzahl der Artikel darstellen, da die Datenbank in INSPEC nicht alle weltweit erscheinenden wissenschaftlichen Journale umfaßt.

Phasen-, Frequenz sowie verallgemeinerter Synchronisation aus Kap. 2 kann im zweiten Teil von Kap. 4 auch bei räumlich ausgedehnten Systemen nachgewiesen werden. In Kap. 5 wird die Anwendung von Synchronisation in der Modellparameterbestimmung an hochdimensionalen und verrauschten Systemen diskutiert. Schließlich wird in Kap. 6 die Arbeit zusammengefaßt und ein Ausblick auf die Zukunft der chaotischen Synchronisation gewagt. 


\section{Einleitung}

\subsection{Konventionen}

In diesem Abschnitt werden kurz die in dieser Arbeit verwendete Notation, Symbole und Konventionen vorgestellt.

Vektoren sind durch arabische Buchstaben in Fettschrift dargestellt, während Matrizen zusätzlich durch doppelten Unterstrich gekennzeichnet sind. In diesem recht jungen Forschungsgebiet haben sich angloamerikanische Namen vieler Phänomene etabliert, welche auch durchgehend benutzt werden, soweit keine zufriedenstellende Übersetzung greifbar ist und/oder der Begriff sich praktisch schon als Eigenname etabliert hat, z.B. „riddled bassins". Diese Begriffe werden zusätzlich mit Anführungsstrichen hervorgehoben. Bei Abkürzungen wird auch durchgängig die in der Literatur übliche angloamerikanische Variante verwendet. Durch diese Konvention soll der Einstieg und die Lesbarkeit von weiterführender Literatur vereinfacht werden.

\section{Häufig verwendete Symbole}

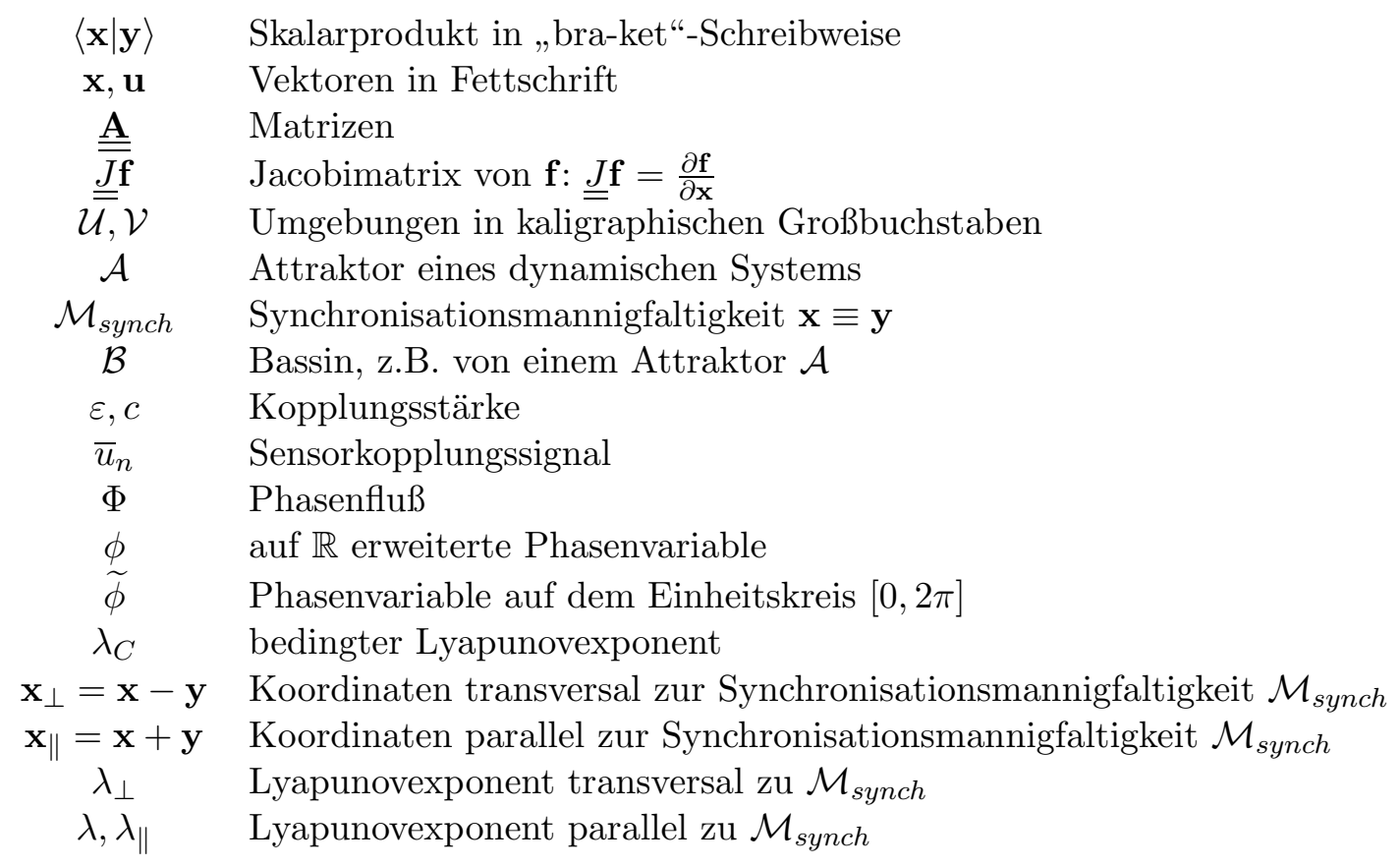




\section{Varianten der Synchronisation gekoppelter (chaot.) dynamischer Systeme}

In diesem Kapitel werden die verschiedenen Synchronisationsvarianten von dynamischen Systemen vorgestellt und diskutiert. In letzter Zeit hat sich ein regelrechter Zoo von verschiedenen Synchronisationstypen gebildet, z.B. identical, complete, phase, lag, frequency, weak, strong, partial, approximative, high-quality, ... Synchronisation, um nur einige zu nennen. Im wesentlichen lassen sich aber alle Varianten in drei Oberklassen zusammenfassen, wobei sich dann die anderen Typen als Synonyme oder Spezialfälle von diesen erweisen. Im Rest des Kapitels werden nun die drei wichtigen Typen der chaotischen Synchronisation nacheinander diskutiert. Der Einfachheit halber beschränken wir uns auf zwei untereinander gekoppelte Systeme, wobei die nachfolgenden Betrachtungen auch für eine beliebige Anzahl gekoppelter Oszillatoren gültig bleiben, welches aber auf dieser Ebene die Argumentation nur erschwert. In Abb. 2.1 ist der Grundauf-

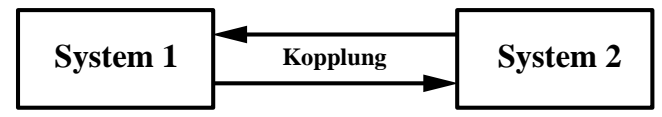

(a) beidseitige Kopplung

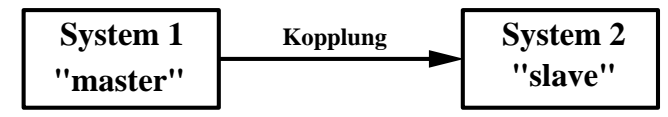

(b) einseitige Kopplung

Abbildung 2.1.: Die beiden verschiedenen Kopplungsarten bei dynamischen Systemen.

bau der Kopplung zweier dynamischer Systeme skizziert. Man benötigt mindestens zwei nicht notwendigerweise identische dynamische Systeme, die in mindestens einer Richtung Informationen austauschen können. Falls die Oszillatoren durch gewöhnliche Differentialgleichungen (ODEs) ${ }^{1}$ modelliert werden können, erhält man im allgemeinsten Fall ein erweitertes dynamisches System

$$
\dot{\mathbf{X}}=\mathbf{F}(\mathbf{X})=\left\{\begin{array}{lll}
\dot{\mathbf{x}} & =\mathbf{f}_{\mathbf{x}}+\mathbf{g}_{\mathbf{x}}(\mathbf{x}, \mathbf{y}), & \mathbf{x} \in \mathbb{R}^{n} \\
\dot{\mathbf{y}} & =\mathbf{f}_{\mathbf{y}}+\mathbf{g}_{\mathbf{y}}(\mathbf{y}, \mathbf{x}), & \mathbf{y} \in \mathbb{R}^{m}
\end{array}\right.
$$

welches in zwei gekoppelte Subsysteme $\mathbf{x}$ und $\mathbf{y}$ zerlegt werden kann. Formal läßt sich jedes System $\mathbf{X}$ in viele Subsysteme zerlegen, welche im ungekoppelten Fall $\mathbf{g}_{\mathbf{x}}=\mathbf{g}_{\mathbf{y}}=0$

\footnotetext{
${ }^{1}$ Ordinary Differential Equations.
} 
Attraktoren $\mathcal{A}_{\mathbf{x}} \subset \mathbb{R}^{n}$ bzw. $\mathcal{A}_{\mathbf{y}} \subset \mathbb{R}^{m}$ besitzen. Durch die Kopplung entsteht ein neues Gesamtsystem $\mathbf{X}$, wodurch auch ein eigenständiger Attraktor $\mathcal{A} \subset \mathbb{R}^{n+m}$ entsteht, der a priori nicht viel mit denen der ungekoppelten System gemein haben muß und dessen Dimensionen auch die der Einzelattraktoren $\mathcal{A}_{\mathbf{x}}$ und $\mathcal{A}_{\mathbf{y}}$ übersteigen kann ${ }^{2}$. Aus diesem Grund muß in den Einzelsysteme $\mathbf{x}$ bzw. $\mathbf{y}$ auch kein eigenständiger Attraktor mehr existieren, so daß sich in der Projektion von $\mathcal{A}$ auf $\mathbf{x}$ bzw. y Trajektorien durchaus schneiden können ${ }^{3}$. In diesen Fällen wird es schwer werden, Synchronisation zu definieren bzw. nachzuweisen und es stellt sich die Frage, ob der Begriff der Synchronisation in solch einen allgemeinen Kontext überhaupt noch Sinn macht. Anstatt ein großes System zu zerlegen, ist es sinnvoller, zwei eigenständige durch Kopplung zusammenzusetzen. Dazu geht man von zwei ungekoppelten selbsterregten Oszillatoren aus, welche nicht notwendig chaotische Dynamik zeigen. Die Kopplung induziert einen gegenseitigen (Abb. 2.1a) bzw. gerichteten (Abb. 2.1b) Informationsaustausch beider Oszillatoren, welcher die Eigendynamik der Einzelsysteme stört und unter gewissen Umständen zur Ausbildung eines neuen Attraktors $\mathcal{A}$ führt, dessen Projektionen auf $\mathbf{x}$ und $\mathbf{y}$ dann auch gemeinsame (synchrone) Eigenschaften besitzen können. Die stärkste Form der Synchronisation tritt auf, falls in den Projektionen auf die Einzelsysteme eigenständige Attraktoren mit einem funktionalen Zusammenhang existieren (siehe Abschn.2.2), wobei dem Spezialfall identischer Zeitentwicklung besondere Bedeutung zukommt (siehe Abschn. 2.1). Es existieren aber auch Synchronisationsvarianten, wo nur spezielle Aspekte der Dynamik synchronisiert sind, während die Einzelsysteme ansonsten praktisch unabhängige Zeitentwicklungen zeigen (siehe Abschn. 2.3).

In diesem Kapitel werden Synchronsiationsphänomene am Beispiel von unidirektional gekoppelten Systemen untersucht. Die meisten Methoden und Resultate bleiben aber auch bei bidirektionaler Kopplung gültig, wobei auf Ausnahmen gesondert hingewiesen wird.

\subsection{Synchronisation identischer Systeme}

Die einfachste Art der chaotischen Synchronisation tritt bei Systemen der exakt gleichen Bauart, d.h. $\mathbf{f}_{\mathbf{x}} \equiv \mathbf{f}_{\mathbf{y}}$ in Glg. (2.1), auf. Bei entsprechend gewählter Kopplung laufen die Trajektorien beider Systeme aufeinander zu und werden asymptotisch vollständig identische Oszillationen $\lim _{t \rightarrow \infty} \mathbf{x}(t)-\mathbf{y}(t)=0$ ausführen. Die Systeme sind dann nicht mehr voneinander zu unterscheiden und heißen identisch synchronisiert. Dies mag auf den ersten Blick etwas überraschen, da sich bei chaotischen Systemen benachbarte Zustände

\footnotetext{
${ }^{2}$ Die Dimension von $\mathcal{A}$ kann i.A. sogar die Summe von $\mathcal{A}_{\mathbf{x}}$ und $\mathcal{A}_{\mathbf{y}}$ übersteigen. Man kann z.B. den Duffingoszillator als ein unidirektional gekoppeltes System, bestehend aus dem harmonischen Antrieb $\ddot{x}=-w_{0}^{2} x$ und einem 2-dimensionalen nichtlinearen getriebenen System $\dot{\mathbf{y}}=\mathbf{f}(\mathbf{y})+x$ mit Fixpunktdynamik für $x=0$, beschreiben. Im ungekoppelten Fall ist das $x$-System 1-dimensional $\left(\sim \cos \left(w_{0} t\right)\right)$ und das y-System 0-dimensional (triviale Fixpunktdynamik). In Parameterbereichen des gekoppelten Systems (Duffing) mit chaotischer Dynamik existiert dann ein chaotischer Attraktor mit Dimension $D>1=D_{x}+D_{y}$.

${ }^{3}$ Dies betrifft natürlich nicht die Existenz und Eindeutigkeit des Attraktors $\mathcal{A}$ des zusammengesetzten Gesamtsystems F.
} 
exponentiell voneinander entfernen müssen, wodurch zeitliche Vorhersagen des Zustandes $\mathbf{x}(t)$ exponentiell ungenauer werden. Das getriebene synchronisierte System soll aber nicht zukünftige Zustände, sondern den aktuellen Zustand des treibenden Systems vorhersagen. Das getriebene System verliert jegliche Information über seinen Anfangszustand und muß die Zeitentwicklung des treibenden Systems kopieren, weswegen die unidirektionale Kopplung oft auch als „master-slave“ Kopplung bezeichnet wird. Dieser Fall ist seit 1990 ausführlich untersucht wurden, siehe z.B. [3, 32, 93, 96, 97, 99, 115], u.a. stimuliert durch die potentielle Anwendung in (sicheren) Kommunikationssystemen.

\section{Definition 2.1 (Identische Synchronisation (IS)) :}

Das gekoppelte System

$$
\begin{aligned}
\dot{\mathbf{x}} & =\mathbf{f}(\mathbf{x})+\mathbf{g}_{\mathbf{x}}(\mathbf{x}, \mathbf{y}) \\
\dot{\mathbf{y}} & =\mathbf{f}(\mathbf{y})+\mathbf{g}_{\mathbf{y}}(\mathbf{x}, \mathbf{y})
\end{aligned}
$$

heißt identisch synchronisierbar, falls:

1. eine kompakte, invariante und transitive Untermannigfaltigkeit

$$
\mathcal{M}_{\text {synch }}:=\left\{\mathbf{x}, \mathbf{y} \in \mathbb{R}^{n} \mid \mathbf{x} \equiv \mathbf{y}\right\} \subset \mathbb{R}^{n},
$$

existiert, welche den synchronisierten (chaotischen) Attraktor $\mathcal{A} \subset \mathcal{M}_{\text {synch }} \subset \mathbb{R}^{n}$ enthält und

2. das Bassin $\mathcal{B}(\mathcal{A}) \subset \mathbb{R}^{n} \times \mathbb{R}^{n}$ eine Umgebung $\mathcal{U}_{\varepsilon}(\mathcal{A})$ beinhaltet, so daß $\forall\left(\mathbf{x}_{0}, \mathbf{y}_{0}\right) \in \mathcal{B}$

$$
\lim _{t \rightarrow \infty}\|\mathbf{x}(t)-\mathbf{y}(t)\|=\left\|\phi^{t}\left(\mathbf{x}_{0}\right)-\phi^{t}\left(\mathbf{y}_{0}\right)\right\|=\mathbf{0}
$$

gilt.

Die identische Synchronisation wird analog für den zeitdiskreten Fall definiert, wobei die Differentialgleichungen durch iterierte Abbildungen $\mathbf{x}^{n+1}=\mathbf{f}\left(\mathbf{x}^{n}\right)$ ersetzt werden. Die Untermannigfaltigkeit $\mathcal{M}_{\text {synch }}$, welche eine $n$-dimensionale Hyperfläche im $\mathbb{R}^{2 n}$ darstellt, wird im weiteren als Synchronisationsmannigfaltigkeit bezeichnet. Die Existenz von $\mathcal{M}_{\text {synch }}$ ist auf jeden Fall gesichert, falls die Kopplungskraft $\mathbf{g}_{\mathbf{x}}(\mathbf{x}, \mathbf{y})=\mathbf{g}_{\mathbf{y}}(\mathbf{x}, \mathbf{y})$ innerhalb von $\mathcal{M}_{\text {synch }}$ verschwindet, was bei allen Kopplungsvarianten in Kap. 3 zutrifft. Die Stabilität dieser Hyperfläche bestimmt die Stabilität des synchronen Zustandes. Die Synchronisationsmannigfaltigkeit ist asymptotisch stabil, falls sie stabil und attraktiv ist, d.h. daß zumindest eine Umgebung um den synchronisierten Attraktor $\mathcal{A}$ im Bassin enthalten ist. Zur Stabilitätsuntersuchung wird üblicherweise der Zustand in die Richtungen parallel $\mathbf{x}_{\|}:=\mathbf{x}+\mathbf{y}$ und senkrecht $\mathbf{x}_{\perp}:=\mathbf{y}-\mathbf{x}$ zu $\mathcal{M}_{\text {synch }}$ zerlegt, siehe Abb. 2.2. Der gemeinsame Phasenraum $\mathbb{R}^{2 n}$ wird dadurch in zwei Teilräume $\mathbf{x}_{\|} \in \mathrm{T} \subset \mathbb{R}^{n}$ und $\mathbf{x}_{\perp} \in \mathrm{N} \subset \mathbb{R}^{n}$ zerlegt. Das System $\dot{\mathbf{x}}_{\|}$bestimmt das Verhalten innerhalb der Synchronisationsmannigfaltigkeit $\mathcal{M}_{\text {synch }}$ und beschreibt im Fall der identischen Synchronisation die Dynamik der synchronisierten Systeme ${ }^{4}$. Die Dynamik senkrecht zu $\mathcal{M}_{\text {synch }}$ und damit

\footnotetext{
${ }^{4}$ Bei bidirektionaler Kopplung muß die Dynamik des synchronen Zustandes i.A. nicht der des ungestörten Systems entsprechen, so daß der synchrone Zustand keine Lösung der ungekoppelten Systeme darstellen muß.
} 


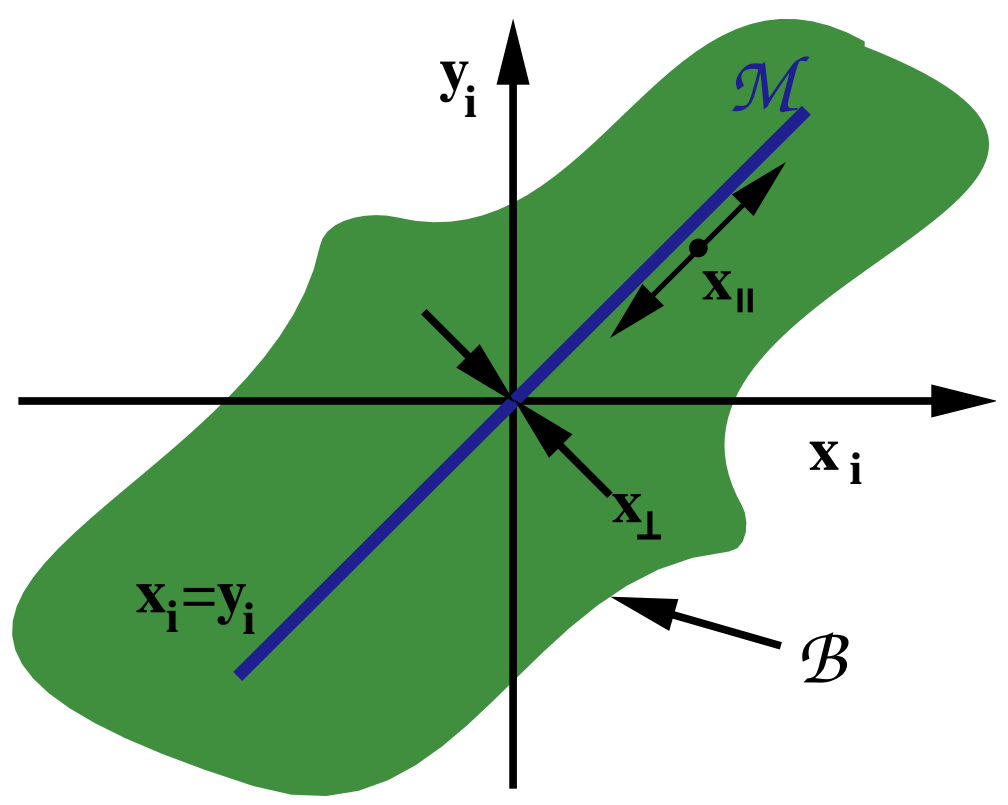

Abbildung 2.2.: Asymptotisch stabile Synchronisationsmannigfaltigkeit $\mathbf{x} \equiv \mathbf{y}$.

die Stabilität des synchronen Zustandes wird durch $\mathbf{x}_{\perp}$ bestimmt, welches anschaulich die Zeitentwicklung des gegenseitigen Abstandes zweier Trajektorien $\mathbf{y}-\mathbf{x}$ beschreibt. Der synchrone Zustand ist asymptotisch stabil, falls $\dot{\mathbf{x}}_{\perp}$ einen asymptotisch stabilen Fixpunkt im Ursprung $\mathbf{0}$ besitzt. Damit ist die Stabilitätsuntersuchung im $2 n$-dimensionalen Phasenraum auf ein Fixpunktproblem im $n$-dimensionalen transversalen Unterraum $\mathbf{x}_{\perp}$ zurückgeführt. Bei unidirektionaler Kopplung $\mathbf{g}_{\mathbf{x}}=0$ ist damit die Stabilität der identischen Synchronisation durch das System

$$
\begin{aligned}
\dot{\mathbf{x}}_{\perp} & =\mathbf{f}(\mathbf{y})-\mathbf{f}(\mathbf{x})+\mathbf{g}_{\mathbf{y}}(\mathbf{x}, \mathbf{y}) \\
& =\mathbf{f}\left(\mathbf{x}_{\perp}+\mathbf{x}\right)-\mathbf{f}(\mathbf{x})+\mathbf{g}_{\mathbf{y}}\left(\mathbf{x}, \mathbf{x}_{\perp}+\mathbf{x}\right)
\end{aligned}
$$

bestimmt. Zur Untersuchung auf asymptotische Stabilität von Glg. (2.5) werden die üblichen Nachweismethoden benutzt, wobei man sorgfältig zwischen notwendigen Kriterien, welche die asymptotische Stabilität von $\mathcal{M}_{\text {synch }}$ nur im Mittel nachweisen, und hinreichenden Kriterien, die die Attraktivität von $\mathcal{M}_{\text {synch }}$ in jedem Punkt sicherstellen können, unterscheiden muß.

- Der Synchronisationsfehler:

Falls alle Variablen $x_{i}, y_{i}$ der Systeme zugänglich sind, dann kann man direkt die Definition von IS (2.1) anwenden und das Verschwinden des Synchronisationsfehlers $e_{i}=x_{i}-y_{i}, i=1, \ldots, n$ in allen Koordinaten für eine hinreichend lange Zeit überprüfen. Der hundertprozentige Nachweis von IS ist zwar streng genommen nur für unendliche Zeiten gesichert, aber praktisch ist sowieso nur der Beobachtungszeitraum relevant. Als ersten Test ist es oft ausreichend, die Synchronisation 
von nur einer Komponente $x_{i}$ mit $y_{i}$ zu überprüfen, da Desynchronisation in den anderen Komponenten praktisch immer auch auf alle anderen zurückkoppelt ${ }^{5}$.

- Bedingte Lyapunovexponenten:

In der nichtlinearen Dynamik ist das Standardkriterium zur Charakterisierung der Stabilitätseigenschaften von Trajektorien das Lyapunovspektrum, welches in leicht modifizierter Form auch zum Nachweis von chaotischer Synchronisation verwenden läßt. Dazu betrachtet man die Linearisierung von Glg. (2.5) um $\mathbf{x}_{\perp}=\mathbf{x}-\mathbf{y}$

$$
\begin{aligned}
\dot{\mathbf{x}}_{\perp} & =\left.\frac{\partial \mathbf{f}(\tilde{\mathbf{y}})}{\partial \tilde{\mathbf{y}}}\right|_{\tilde{\mathbf{y}}=\mathbf{x}} \mathbf{x}_{\perp}+\left.\frac{\partial \mathbf{g}(\mathbf{x}, \tilde{\mathbf{y}})}{\partial \tilde{\mathbf{y}}}\right|_{\tilde{\mathbf{y}}=\mathbf{x}} \mathbf{x}_{\perp}+\mathcal{O}\left(\mathbf{x}_{\perp}^{2}\right) \\
& =\left.\underline{\underline{\mathbf{J}_{\mathbf{y}}}}\right|_{\mathbf{x}} \mathbf{x}_{\perp}+\left.\underline{\underline{\mathbf{J}_{\mathbf{y}}} \mathbf{g}}\right|_{\mathbf{x}} \mathbf{x}_{\perp}+\mathcal{O}\left(\mathbf{x}_{\perp}^{2}\right) .
\end{aligned}
$$

Man beachte, daß Glg. (2.6) neben $\mathbf{x}_{\perp}$ auch noch von der Trajektorie des treibenden Systems x abhängt, weswegen die resultierenden Stabilitätsexponenten $\lambda_{\perp}$ bedingte Lyapunovexponenten ${ }^{6} \lambda_{C}$ genannt werden. Falls der größte und damit alle bedingten Lyapunovexponenten negativ sind, ist die Synchronisationsmannigfaltigkeit $\mathcal{M}_{\text {synch }}$ im Mittel attraktiv, weswegen diese Bedingung offensichtlich notwendig für IS ist. Daß die Bedingung nicht hinreichend sein kann, macht man sich mit folgender Überlegung klar. $\lambda_{\perp}<0$ besagt, daß die Anziehung benachbarter Trajektorien die Abstoßung überwiegt, so daß kurze Abschnitte mit einem Auseinanderlaufen der Systeme in anderen Bereichen überkompensiert werden. Falls aber ausgedehnte Bereiche auf $\mathcal{M}_{\text {synch }}$ existieren, die zwar stark abstoßend sind, aber nur sehr selten von der Trajektorie angesteuert werden ${ }^{7}$, so kann die Synchronisation kurzzeitig vollständig zusammenbrechen und Intermittenz tritt auf. Aufgrund ihrem kleinen Maß haben diese Bereiche aber kaum Einfluß auf $\lambda_{\perp}$, so daß $\mathcal{M}_{\text {synch }}$ im Mittel stabil bleibt. Falls aber $\lambda_{\perp}$ deutlich kleiner Null ist, wird solch ein Verhalten sehr unwahrscheinlich, weswegen die Negativität von $\lambda_{\perp}$ trotz allem ein wichtiger Indikator für IS bleibt. Anzumerken ist noch, daß die bedingten Lyapunovexponenten nur Aussagen über die lineare Stabilität von $\mathcal{M}_{\text {synch }}$ liefern können und damit nur die Stabilität in einer Umgebung von $\mathcal{M}_{\text {synch }}$ beschreiben.

- Lineare Stabilitätsuntersuchung:

In manchen Fällen, wo die lineare Matrix aus Glg. (2.6) nur konstante Koeffizienten besitzt bzw. wo der zeitabhängige Anteil x vom treibenden System abgeschätzt werden kann, kann auch der Realteil des größten Eigenwertes des Systems zur Stabilitätsanalyse herangezogen werden.

\footnotetext{
${ }^{5}$ Meist wird man nicht direkt eine Zustandskomponente sondern eine Funktion des Zustandes $s=h(\mathbf{x})$ messen und als Kopplungssignal verwenden, so daß die Synchronisation von $s=h(\mathbf{x})$ mit $s=h(\mathbf{y})$ als erster Indikator für IS benutzt werden wird. Ein Gegenbeispiel für diesen Test liefert [40], wo nur Teile der Zustände $\mathbf{x}$ und $\mathbf{y}$ miteinander synchronisieren. Solch ein Szenario ist aber sehr selten und wird bei praktisch allen Systemen nicht beobachtet.

6 ,conditonal lyapunov exponents“

${ }^{7}$ Solch ein Verhalten tritt bevorzugt in der Umgebung von instabilen periodischen Orbits auf [46].
} 
- Lyapunovfunktionen:

Eine hinreichende Ausage über die Stabilität des synchronen Zustandes erhält man, wenn man eine Lyapunovfunktion $V\left(\mathbf{x}_{\perp}\right)$ für das System (2.5) bzw. (2.6) konstruieren kann. Falls die Lyapunovfunktion $V\left(\mathbf{x}_{\perp}\right)$ positiv definit und deren Ableitung negativ definit ist, so ist asymptotische Stabilität von IS garantiert und der Gültigkeitsbereich von $V$ liefert zusätzlich noch eine untere Abschätzung des Bassins $\mathcal{B}$. Eine Lyapunovfunktion liefert zwar eine hinreichende Stabilitätsaussage, ist aber i.A. schwer zu konstruieren bzw. zu finden, weswegen diese Methode (leider) nur selten angewandt werden kann.

- Stabilität invarianter Untermengen:

Bei der Diskussion zum bedingten Lyapunovexponenten wurden schon die lokalen Instabilitäten von $\mathcal{M}_{\text {synch }}$, verursacht durch instabile periodische Orbits (UPOs) ${ }^{8}$, angedeutet. Andererseits können bei ergodischen Systemen dynamische Invarianten, wie z.B. fraktale Dimensionen, Lyapunovexponenten, etc., durch eine Entwicklung des Attraktors nach den UPOs beliebig gut approximiert werden [5, 142]. Die UPOs liegen dicht im chaotischen Attraktor, so daß jede Trajektorie im Laufe ihrer Zeitentwicklung jedem UPO im Attraktor beliebig nahe kommt. Falls nun zwei gekoppelte chaotische Systeme identisch synchronisieren, dann müssen dies auch alle in ihren Attraktor eingebetteten UPOs tun. In der Arbeit [46] wurde gezeigt, daß maximale Zeitmittelwerte typischerweise auf UPOs niedriger Periode angenommen werden. Zum Nachweis von IS waren wir oben an dem größten Lyapunovexponenten interessiert, welcher aus diesem Grund meist auf niederperiodischen UPOs seinen Maximalwert annimmt ${ }^{9}$. Das bedeutet aber, falls alle niederperiodischen UPOs im Attraktor synchronisieren, so muß es eine typische Trajektorie schon recht tun. Streng genommen erhält man eine hinreichende Aussage nur bei Heranziehung von allen UPOs, aber aufgrund [46] kann IS durch Betrachtung von den niederperiodischen UPOs fast immer garantiert werden. Eine ausführliche Behandlung des Nachweis von IS mittels verschiedener invarianter Maße findet man in $[18,33]$.

Als Beispiel betrachten wir den Rössleroszillator [112]

$$
\begin{aligned}
& \dot{x}_{1}=2+x_{1}\left(x_{2}-4\right) \\
& \dot{x}_{2}=-x_{1}-x_{3} \\
& \dot{x}_{3}=x_{2}+0.45 x_{3},
\end{aligned}
$$

welcher bei diesen Parameterwerten den chaotischen Attraktor in Abb. 2.3a besitzt. Mit $s_{x}=x_{2}-x_{1}$ und entsprechend $s_{z}=z_{2}-z_{1}$ als Kopplungssignal wollen wir nun zeigen,

${ }^{8}$ „Unstable Periodic Orbits“

${ }^{9}$ Ein UPO ist eine instabile aber gültige und invariante Lösung des Systems, d.h. falls das System mit Anfangswerten, welche exakt auf einem UPO liegen, initialisiert wird, kann es diesen nicht mehr verlassen. Man kann damit z.B. den bedingten Lyapunovexponenten (in diesem Zusammenhang besser Floquetmultiplikator) bezüglich dieses UPOs berechnen und damit die asymptotische Stabilität bezüglich dieses invarianten UPO-Maßes nachweisen. 

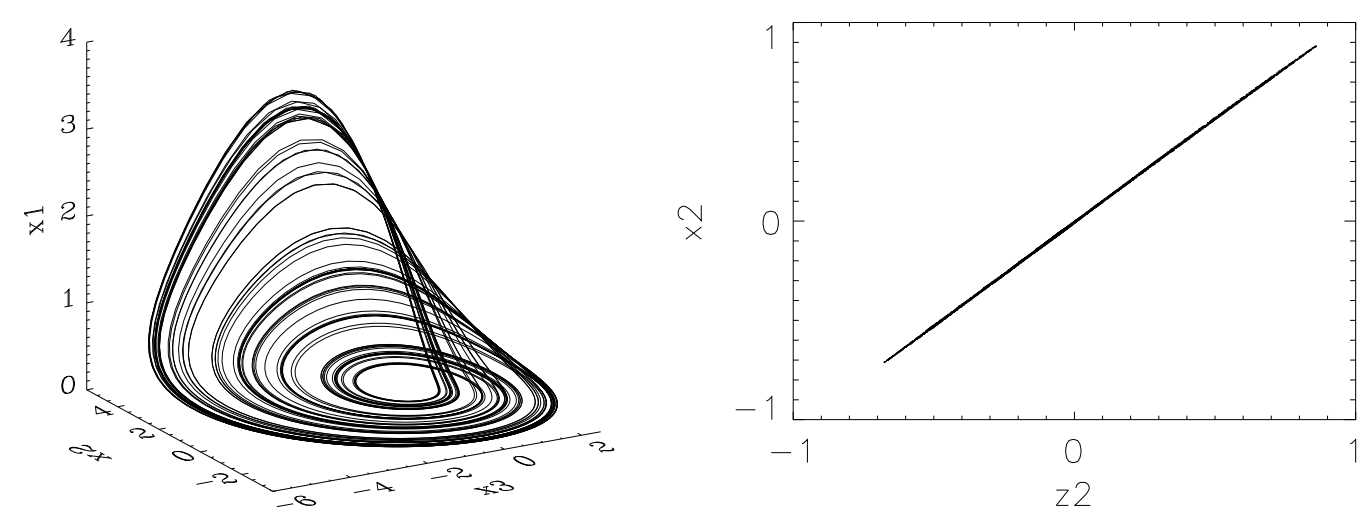

Abbildung 2.3.: a)Der Attraktor des Rössler-Oszillators und b) Nachweis von IS, wobei $x_{2}$ gegen $z_{2}$ aufgetragen ist.

daß das System

$$
\begin{aligned}
& \dot{z}_{1}=2+z_{1}\left(z_{2}-4\right)+\left[s_{z}-s_{x}\right] z_{2} \\
& \dot{z}_{2}=-z_{2}-z_{3}+s_{x} \\
& \dot{z}_{3}=z_{2}+0.45 z_{3}
\end{aligned}
$$

unter diesen Antrieb mit (2.7) synchronisiert. Die Dynamik von $\mathbf{e}:=\mathbf{x}_{\perp}$ aus Glg. 2.5 wird durch

$$
\begin{aligned}
& \dot{e}_{1}=-4 e_{1}+e_{2}\left(x_{2}+y_{2}-s_{x}\right) \\
& \dot{e}_{2}=-e_{2}-e_{3} \\
& \dot{e}_{3}=e_{2}+0.45 e_{3} .
\end{aligned}
$$

bestimmt. Die $e_{1}$-Koordinate ist von den restlichen entkoppelt, so daß man zuerst das $\left(e_{2}-e_{3}\right)$-Subsystem alleine betrachten darf. Eine lineare Stabilitätsanalyse liefert die Eigenwerte $\lambda_{2,3}=-0.275 \pm i \sqrt{0.474}$, womit das Subsystem asymptotisch stabil ist und $e_{2}$ und $e_{3}$ für $t \rightarrow \infty$ gegen 0 konvergieren. Die Gleichung für $e_{1}$ reduziert sich dann auf $\dot{e}_{1}=-4 e_{1}$, wodurch auch $e_{1}$ verschwindet, so daß $\mathbf{x}_{\perp}$ einen asymptotisch stabilen Fixpunkt im Ursprung besitzt und $\mathcal{M}_{\text {synch }}$ (linear) stabil ist.

\subsubsection{Der Übergang}

Synchronisation chaotischer Oszillatoren ist ein Schwellenphänomen, welches eine minimale aber endliche Kopplungskraft zum Erreichen des synchronen Zustandes benötigt. Um dies zu verdeutlichen, betrachten wir zwei bidirektional dissipativ gekoppelte ODEs

$$
\begin{aligned}
\dot{\mathbf{x}} & =\mathbf{f}(\mathbf{x})+\varepsilon(\mathbf{y}-\mathbf{x}) \\
\dot{\mathbf{y}} & =\mathbf{f}(\mathbf{y})+\varepsilon(\mathbf{x}-\mathbf{y})
\end{aligned}
$$




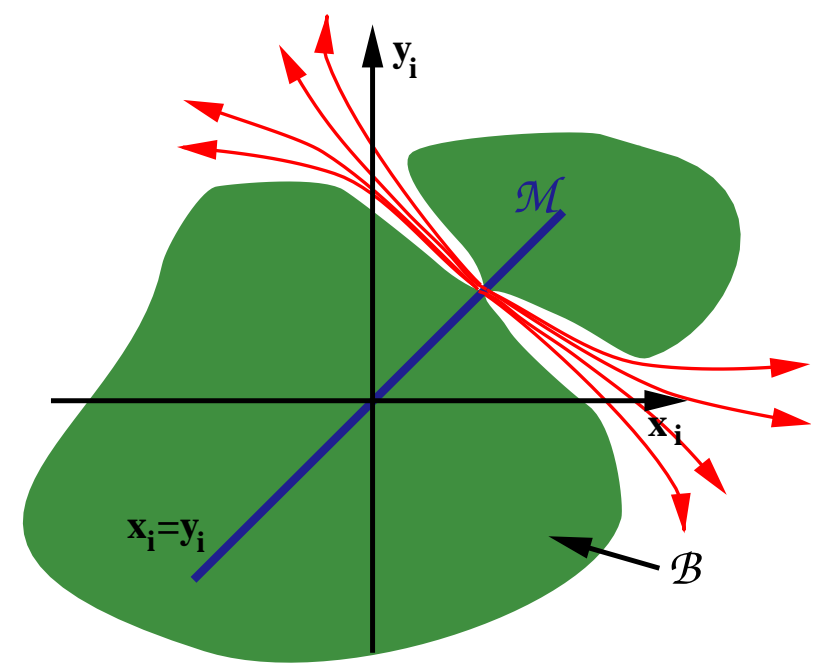

Abbildung 2.4.: Synchronisationsmannigfaltigkeit $\mathbf{x} \equiv \mathbf{y}$ mit lokaler Instabilität, so daß $\mathcal{M}_{\text {synch }}$ nur im Mittel stabil ist.

wobei $\varepsilon$ die Stärke Kopplung bestimmt und das System $\dot{\mathbf{x}}=\mathbf{f}(\mathbf{x})$ einen chaotischen Attraktor $\mathcal{A}_{\mathbf{x}}$ besitzt. Man kann leicht zeigen, daß die Synchronisationsmannigfaltigkeit $\mathbf{x}=\mathbf{y}$ für $\varepsilon>\frac{1}{2} \lambda_{\|}$(im Mittel) stabil ist [32], wobei $\lambda_{\|}$den größten Lyapunovexponenten von $\mathcal{A}_{\mathbf{x}}$ bezeichnet. Zur identischen Synchronisation wird aber asymptotische Stabilität des synchronisierten Attraktors $\mathcal{A} \subset \mathcal{M}_{\text {synch }}$ benötigt. Dazu rufen wir uns erstmal einige Definitionen von Attraktoren in Erinnerung. Sei $\mathcal{A}$ eine kompakte, invariante und transitive Menge des Systems (2.2). Das Bassin $\mathcal{B}(\mathcal{A})$ ist die Punktmenge, welche für $t \rightarrow \infty$ auf $\mathcal{A}$ abgebildet wird. Nach [74] ist dann $\mathcal{A}$ ein asymptotisch stabiler Attraktor, falls er Lyapunov stabil ist und $\mathcal{B}(\mathcal{A})$ eine Umgebung von $\mathcal{A}$ enthält ${ }^{10}$. Falls die Synchronisationsmannigfaltigkeit einen asymptotisch stabilen Attraktor enthält, tritt damit nach Def. 2.1 IS auf. $\mathcal{A}$ heißt Milnor-Attraktor, falls $\mathcal{B}(\mathcal{A})$ nur positives Lebesguemaß besitzt. Der asymptotisch stabile Attraktor ist damit ein Spezialfall des Milnor-Attraktors. Ein Milnor-Attraktors $\mathcal{A}$ besitzt ein lokal zerlöchertes („riddled“) Bassin, falls ein $d>0$ existiert, so daß $\forall \mathbf{x} \in \mathcal{B}(\mathcal{A})$ eine Umgebung um $\mathbf{x}$ eine Punktmenge mit positiven Maß enthält, dessen Trajektorien einen Abstand d von $\mathcal{A}$ im Laufe der Zeitentwicklung überschreiten. Schließlich ist $\mathcal{A}$ ein chaotischer Sattel, falls $\mathcal{A}$ nicht mehr Lyapunov stabil ist, und damit transversal instabil wird. Alle diese Varianten werden bei der chaotischen Synchronisation beobachtet. Falls man einen Parameter, welcher die Dissipation transversal zu $\mathcal{M}_{\text {synch }}$ bestimmt ( $\varepsilon$ aus Glg. (2.10)), langsam erhöht, beobachtet man folgendes Verhalten:

- $\varepsilon<\varepsilon_{1}$ : $\mathcal{A}$ ist ein chaotischer Sattel, so daß keine IS auftritt. Der transversale Lyapunovexponent $\lambda_{\perp}$ ist für jede Trajektorie innerhalb $\mathcal{M}_{\text {synch }}$ positiv (bezüglich aller Maße).

\footnotetext{
${ }^{10}$ Lyapunov stabil bedeutet, daß zu jeder Umgebung $\mathcal{U}(\mathcal{A})$ eine weitere Umgebung $\mathcal{V}(\mathcal{A})$ existiert, so daß $\forall \mathbf{x} \in \mathcal{V}$ ein $t_{0}$ existiert mit $\Phi_{t}(\mathbf{x}) \in \mathcal{U}$ für $t>t_{0}$.
} 
- $\varepsilon_{1} \leq \varepsilon<\varepsilon_{2}$ : $\mathcal{A}$ ist ein Milnor-Attraktor und der transversale Lyapunovexponent $\lambda_{\perp}$ ist negativ bezüglich einer typischen Trajektorie (natürliches Maß). Es besteht damit zwar eine positive Wahrscheinlichkeit, daß ein beliebiger Punkt $\mathbf{x}_{0}$ auf den synchronisierten Attraktor führt, wobei aber beliebig kleine Störungen die Synchronisation zerstören werden.

- $\varepsilon_{2} \leq \varepsilon<\varepsilon_{3}: \mathcal{A}$ ist ein Milnor-Attraktor mit einem lokalen „riddled“ Bassin, wobei wieder $\lambda_{\perp}<0$ ist, aber Trajektorien im Attraktor mit $\lambda_{\perp}>0$ existieren können. Zum Beispiel kann $\mathcal{A}$ bezüglich aller Maße transversal stabil sein, bis auf die Ausnahme eines in $\mathcal{A}$ eingebetteten UPOs, welcher innerhalb von $\mathcal{M}_{\text {synch }}$ Lebesguemaß Null hat und somit auch mit Wahrscheinlichkeit Null erreicht wird. Transversal zu $\mathcal{M}_{\text {synch }}$ existiert nun eine Arnoldzunge (siehe Abb. 2.4), so daß bei Anwesenheit von beliebig kleinem Rauschen eine kleine aber positive Wahrscheinlichkeit besteht, daß eine Trajektorie in der Nähe des UPOs in die Arnoldzunge gerät und sich von $\mathcal{A}$ möglicherweise weit entfernt ${ }^{11}$. Falls kein weiterer Attraktor im Phasenraum existiert, kehrt die Trajektorie nach kurzer Zeit wieder in die Nähe von $\mathcal{A}$ zurück und man beobachtet lange synchrone Phasen, welche durch seltene und kurze intermittente Ausbrüche unterbrochen werden. Existiert ein weiterer Attraktor im Phasenraum, so kehrt die Trajektorie, je nach dessen transversalen Stabilitätseigenschaften, überhaupt nicht, oder erst nach langen Zeiten, in die Nähe des synchronen Zustandes zurück.

- $\varepsilon_{3} \leq \varepsilon: \mathcal{A}$ ist ein asymptotisch stabiler Attraktor, so daß $\lambda_{\perp}$ für jede Trajektorie oder bezüglich aller Maße negativ ist und IS auch gegenüber kleinem Rauschen stabil ist.

Der Vorzeichenwechsel des transversalen Lyapunovexponenten $\lambda_{\perp}$ (bezüglich des natürlichen Maßes) wird oft auch als „blowout“-Bifurkation bezeichnet [81]. Allgemein wird durch Erhöhung der Dissipation $\varepsilon$ transversal zu $\mathcal{M}_{\text {synch }}$ der identisch synchrone Zustand bezüglich immer weiterer Maße transversal stabil, wodurch die synchronen Phasen immer länger werden, bis IS asymptotisch stabil wird. Im letzten Abschnitt wurde schon auf die Sonderstellung von niederperiodischen UPOs an der Stabilität von IS hingewiesen, welche fast immer bei steigenden $\varepsilon$ als letztes transversal stabil werden. Für eine tiefergehende Behandlung dieser interessanten Phänomene möchten wir auf die Arbeiten $[4,7,81,100,135]$ verweisen.

\footnotetext{
${ }^{11}$ Man benötigt ein (beliebig) kleines Rauschen damit die Trajektorie nicht exakt in der Synchronisationsmannigfaltigkeit $\mathcal{M}_{\text {synch }}$ liegt, welche sonst $\mathcal{M}_{\text {synch }}$ aufgrund der Invarianz nicht mehr verlassen kann. Falls die Trajektorie sich außerhalb von $\mathcal{M}_{\text {synch }}$ befindet und sich dem synchronen Zustand exponentiell nähert (sie kann ihn aber nie exakt Erreichen), so wird sie für $t \rightarrow \infty$ der Arnoldzunge beliebig nahe kommen, so daß dann auch wieder Ausbrüche auftreten. Im Computerexperiment muß aber immer ein wenig Rauschen addiert werden, da aufgrund der begrenzten Rechengenauigkeit, die Systeme nach einiger Zeit perfekt synchronisiert sind, wodurch in Abwesenheit von Rauschen perfekte IS bei Anwesenheit von transversalen Instabilitäten möglich ist!
} 


\subsubsection{Einfluß von Rauschen}

Bei experimentellen Systemen liegen praktisch nie zwei exakt identische Systeme zur Synchronisation vor, wobei die gemessenen Signale darüberhinaus noch verrauscht sein werden. Damit stellt sich die Frage, in wie weit sich leichte Parameterunterschiede und Rauschen auf die Qualität der Synchronisation auswirken. Perfekte Synchronisation $\mathbf{x} \equiv \mathbf{y}$ ist dann sicherlich nicht mehr erreichbar. In diesem Fall spricht man von identischer Synchronisation, falls der Synchronisationsfehler nach oben beschränkt bleibt $\|e\|=\|\mathbf{x}-\mathbf{y}\|<\eta$, wobei $\eta$ deutlich kleiner als der Attraktordurchmesser sein sollte. Oft wird dies noch etwas abgeschwächt, in dem man nur die Beschränktheit des mittleren Synchronisationsfehlers $\left[\left\langle|\mathbf{e}|^{2}\right\rangle\right]^{\frac{1}{2}}<\eta$ fordert. Eine systematische Untersuchung der Effekte von Rauschen und Drift in den Systemparametern wurde in [19] durchgeführt. Es wurde vorrausgesetzt, daß eine kleine Parameteränderung keine drastische Veränderung der Systemdynamik verursacht, was z.B. in der Nähe von Bifurkationen vorkommen kann. Weiterhin soll die Kopplung so gewählt sein, daß der synchronisierte Attraktor asymptotisch stabil ist und sich in hinreichender Entfernung von der „blowout"-Bifurkation befindet, so daß transversale Instabilitäten keine Rolle spielen. Falls dies erfüllt ist, kann man analytisch zeigen, daß sich der mittlere Synchronisationsfehler $\left[\left\langle|\mathbf{e}|^{2}\right\rangle\right]^{\frac{1}{2}}=\left[\left\langle|\mathbf{x}-\mathbf{y}|^{2}\right\rangle\right]^{\frac{1}{2}}$, bei kleinen Parameterunterschieden und additivem Rauschen der Stärke $\sigma$ auf dem Meßsignal, durch die Beziehung

$$
\bar{e}=\left[\left\langle|\mathbf{e}|^{2}\right\rangle\right]^{\frac{1}{2}}=\left[A^{2}+(\sigma B)^{2}\right]^{\frac{1}{2}}
$$

ausdrücken läßt. Die Konstanten $A$ und $B$ sind systemabhängig, wobei aber $A$ nur von den Parameterunterschieden und $B$ nur von der Kopplungsstärke und dem verwendeten Rauschtyp (z.B. weißes oder Gauß'sches Rauschen) abhängen. Bei identischen Systemen $(A=0)$ skaliert der mittlere Synchronisationsfehler $\bar{e}$ somit linear mit der Rauschamplitude bzw. der Rauschleistung. In Experimenten ist somit IS auch dann erreichbar, falls das additive Rauschen nicht von der Größenordnung des Kopplungssignales ist. Bei unverrauschten Systemen $(\sigma=0)$ gleicher Struktur ist IS auch stabil gegen kleine Parameterunterschieden bzw. eine nicht zu große Variabilität in den Systemparametern. Im allgemeinen Fall ist durch Glg. (2.11) die strukturelle Stabilität von chaotischer Synchronisation gegenüber kleinen Störungen gesichert, welches in [19] anhand numerischer und experimenteller Systeme auch überprüft wurde. Die oben gemachte Definition von IS durch den mittleren Synchronisationsfehler sollte besser als approximative oder schwache Synchronisation bezeichnet werden, da der mittlere Synchronisationsfehler auch beim Vorhandensein von transversalen Instabilitäten bzw. seltener Intermitttenz beschränkt bleiben kann, so daß diese Größe eigentlich nicht zum Nachweis von asymptotischer Stabilität geeignet ist. Besser ist es, den instantanen Synchronisationsfehler $\|e(t)\|=\|\mathbf{x}(t)-\mathbf{y}(t)\|<\eta$ zu überwachen, so daß zumindest Intermittenzeffekte ausgeschlossen werden können. Experimentell werden beide Kriterien zum Nachweis von IS benutzt, wo je nach Anwendung abgewogen werden muß, ob Synchronisation im Mittel oder zu jeden Zeitpunkt ausreichend ist. 


\subsection{Verallgemeinerte Synchronisation}

Bis jetzt wurde die Synchronisation von zwei Systemen exakt gleicher Bauart diskutiert. Synchronisation ist aber auch bei gekoppelten Systemen von vollständig verschiedener Natur möglich, wobei dann i.A. keine simple invariante Mannigfaltigkeit der Form $\mathbf{x}=\mathbf{y}$ mehr existiert und die Beziehung zwischen den Systemen weitaus komplizierter sein kann. Wir betrachten zwei unidirektional gekoppelte ODEs

$$
\begin{aligned}
\dot{\mathbf{x}} & =\mathbf{f}_{\mathbf{x}}(\mathbf{x}) \\
\dot{\mathbf{y}} & =\mathbf{f}_{\mathbf{y}}(\mathbf{y})+\mathbf{g}(\mathbf{y}, \mathbf{x})
\end{aligned}
$$

mit $\mathbf{x} \in \mathbb{R}^{n}$ und $\mathbf{y} \in \mathbb{R}^{m}$. Das System (2.12) entwickelt sich in einem $n+m$-dimensionalen Phasenraum $\mathbb{R}^{n} \times \mathbb{R}^{m}$, wobei im ungekoppelten Fall $\mathbf{g}=0$ beide Systeme einen eigenständigen nicht notwendigerweise chaotischen Attraktor besitzen mögen. Das gekoppelte System (2.12) besitzt die Eigenschaft von verallgemeinerter Synchronisation $^{12}$ (GS), falls die Attraktoren $\mathcal{A}_{1}, \mathcal{A}_{2}$ der Einzelsysteme durch eine stetige Abbildung $\mathbf{y}=\mathbf{H}(\mathbf{x})$ ineinander transformiert werden können. Die Dynamik im $n+m$-dimensionalen gemeinsamen Phasenraum ist dann auf einen Attraktor $\mathcal{A} \subset \mathbb{R}^{\max \{n, m\}}$ beschränkt, dessen Projektionen auf $\mathbf{x}$ bzw. y auf die Attraktoren der Einzelsysteme $\mathcal{A}_{1}=\pi_{\mathbf{x}}\{\mathcal{A}\}$ bzw. $\mathcal{A}_{2}=\pi_{\mathbf{y}}\{\mathcal{A}\}$ führen. Diese Definition wurde im Prinzip schon 1986 von Afraimovich [3] vorgeschlagen und in einem russischen Journal veröffentlicht, weswegen sie wahrscheinlich zu dieser Zeit von der breiten wissenschaftlichen Gemeinschaft (noch) nicht wahrgenommen wurde. Erst 10 Jahre später rückte GS wieder in den Fokus der (Synchronisations) Forschung [2, 61, 114] und wird seitdem intensiv studiert. Die obige Definition von GS ist nicht die Allgemeinste und wird aus mehreren Gründen in Abschn. 2.2.2 noch verallgemeinert werden. Aus Verständnisgründen wollen wir uns hier aber dem allgemeinen Fall langsam annähern, wobei die Probleme der obigen Definition nach und nach aufgedeckt werden. Verallgemeinerte Synchronisation kann nicht nur bei unterschiedlichen Systemen, sondern auch zwischen identischen Systemen auftreten [91]. Das GS nicht nur von akademischem Interesse ist, wurde z.B. in [25] gezeigt, wo GS bei linearen rekursiven Filtern nachgewiesen und intensiv untersucht wurde. Der Name verallgemeinerte Synchronisation ist etwas unglücklich gewählt ${ }^{13}$, weil GS sicherlich nicht die allgemeinste Art von synchronen Eigenschaften zweier gekoppelter Systeme beschreiben kann. Der Name hat sich aber inzwischen in der Literatur fest etabliert. Die Beziehung $\mathbf{y}=\mathbf{H}(\mathbf{x})$ definiert eine invariante Untermannigfaltigkeit im gemeinsamen Phasenraum $\mathbb{R}^{n} \times \mathbb{R}^{m}$ beider Systeme. Im Gegensatz zu IS ist in diesem Kontext die Existenz dieser Synchronisationsmannigfaltigkeit nicht a priori gesichert und muß zuerst nachgewiesen werden (Abschn. 2.2.1). Die pure Existenz und Stabilität liefern aber noch keine Aussagen über die funktionale Form, die auch nur selten explizit angegeben werden kann (siehe Abschn. 2.2.3), wobei sie in bestimmten Fällen aber mit Hilfe der nichtlinearen Zeitreihenanalyse (durch Modellbildung) approximiert werden kann [16].

\footnotetext{
12 ,generalized synchronization“

${ }^{13}$ Der Ursprung des Namens hängt wahrscheinlich damit zusammen, daß GS IS als Spezialfall beinhaltet und deswegen eine Verallgemeinerung dieser speziellen Synchronisationsvariante darstellt.
} 


\section{Varianten der Synchronisation gekoppelter (chaot.) dynamischer Systeme}

Ein einfaches Beispiel, wo die Existenz einer invarianten Mannigfaltigkeit und deren Stabilität gesichert ist, kann konstruiert werden, indem man zwei identische Systeme, welche IS zeigen, betrachtet. Werden die Variablen des getriebenen Systmes durch eine stetig differenzierbare Abbildung transformiert, so entsteht ein neues äquivalentes System. Dynamische Invarianten und Stabilitätseigenschaften bleiben aber von solchen Transformationen unbeeinflußt, so daß der neue Attraktor des getriebenen Systems eine Funktion des treibenden bleibt, wobei die Funktion $\mathbf{H}$ und damit die invariante Mannigfaltigkeit explizit durch die stetig differenzierbare Kooordinatentransformation gegeben ist. Weitere Probleme stellen der Nachweis der Eindeutigkeit und der Differenzierbarkeit dar, welche in Abschn. 2.2.2 und Abschn. 2.2.3 gesondert behandelt werden.

\subsubsection{Nachweis}

Im Fall von unidirektionaler Kopplung wird dem getriebenen System, wie bei IS, die Dynamik des treibenden Systems (bzw. eine Funktion davon) aufgezwungen. Das getriebene System verliert im Falle von GS daher jegliche Information über seinen Anfangszustand $\mathbf{y}(0)$ und hat keine eigenen Freiheitsgrade mehr. Dies ist äquivalent dazu, daß das getriebene System $\dot{\mathbf{y}}=\mathbf{f}_{\mathbf{y}}(\mathbf{y})+\mathbf{g}(\mathbf{y}, \mathbf{x})$ aus Glg. (2.12) asymptotisch stabil für alle $\mathbf{y}$ im Attraktor des getriebenen Systems ist [61], wodurch auch indirekt die Existenz der Synchronisationsmannigfaltigkeit nachgewiesen ist. Anzumerken ist, daß durch asymptotische Stabilität des getriebenen Systems keine spezielle Eigenschaften der Mannigfaltigkeit, wie z.B. Differenzierbarkeit, nachgewiesen werden können. Weiterhin treten Probleme bei Systemen mit subharmonischer Einrastung (siehe Abschn 2.2.2) auf, wo i.A. mehrere Bassins existieren, gegenüber denen die asymptotische Stabilität des getriebenen Systems nachgewiesen werden muß. Im Allgemeinen muß die asymptotische Stabilität gegenüber allen invarianten Maßen auf dem synchronisierten Attraktor $\mathcal{A}$ nachgewiesen werden, wozu wieder die Werkzeuge aus Abschn. 2.1 zur Verfügung stehen. Der Nachweis von GS mittels bedingter Lyapunovexponenten (bezüglich dem natürlichen Maß) ist hier aber weitaus problematischer als bei IS. Während bei IS kurzzeitige Zusammenbrüche der Synchronisation aufgrund transversaler Instabilitäten oft einfach durch Vergleich des Kopplungssignales mit der korrespondierenden Größe aus dem getriebenen System zumindest entdeckt werden können, ist dies wegen der unbekannten Form der Transformation $\mathbf{H}$ bei GS nicht mehr möglich. Bei unidirektionaler Kopplung können solche Ereignisse aber auch ohne Kenntnis der expliziten Form der Transformation $\mathbf{H}$ nachgewiesen werden, wozu ein zusätzliches Hilfssystem benötigt wird. Falls das getriebene System asymptotisch stabil ist und GS auftritt, hängt $\mathbf{y}$ nur von $\mathbf{x}$ ab, d.h. aber, daß eine identische Kopie des getriebenen Systems $\dot{\mathbf{z}}=\mathbf{f}_{\mathbf{y}}(\mathbf{z})+\mathbf{g}(\mathbf{z}, \mathbf{x})$, unter demselben Antrieb $\mathbf{x}(t)$, der exakt gleichen Dynamik wie $\mathbf{y}$ folgen muß ${ }^{14}$. Falls $\mathbf{y}$ also mit dem Hilfssystem $\mathbf{z}$ identisch synchronisiert, so ist $\dot{\mathbf{y}}=\mathbf{f}_{\mathbf{y}}(\mathbf{y})+\mathbf{g}(\mathbf{y}, \mathbf{x})$ asymptotisch stabil und GS tritt auf. Das Hilfssystem wird in der Literatur als „auxiliary system“ bezeichnet [2] und liefert eine einfache Möglichkeit GS auch in Experimenten nachzuweisen, wo die Anfertigung

\footnotetext{
${ }^{14}$ In Abschn. 2.2.2 wird gezeigt werden, daß die Transformation $\mathbf{H}$ i.A. nicht eindeutig sein muß, welches mehrere Bassins für das „auxiliary“ System impliziert. IS von y mit dem „auxiliary“ System z muß dann bezüglich aller Bassins nachgewiesen werden.
} 
einer identischen Kopie möglich ist. Durch leichte Parameterunterschiede und Addition von Rauschen kann zusätzlich noch die Robustheit der Synchronisationsmannigfaltigkeit $\mathbf{H}$ gegenüber kleinen Störungen überprüft werden. Falls nur Information von $\mathbf{x}$ und $\mathbf{y}$ in Form von (skalaren) Zeitreihen vorliegt, kann man immer noch mit nächster Nachbarstatistik [114] oder Ähnlichkeitsmaßen [6, 120] Evidenz für die Existenz einer funktionalen Beziehung zwischen den gekoppelten Systemen erhalten.

Als Beispiel soll nun GS zwischen dem Chua-Schwingkreis mit dem Lorenzsystem unter-

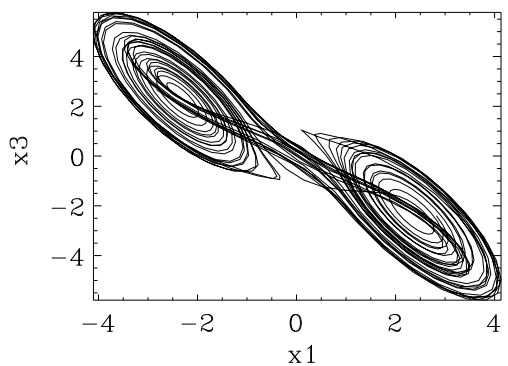

(a) $x_{1}$ gegen $x_{3}$.

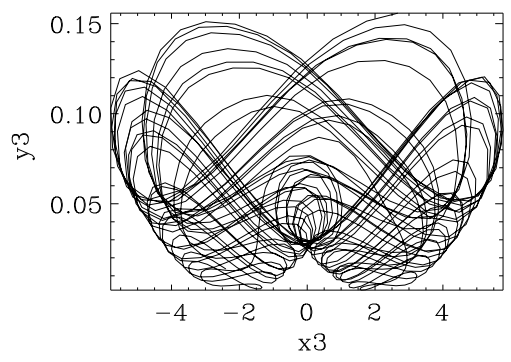

(c) $x_{3}$ gegen $y_{3}$

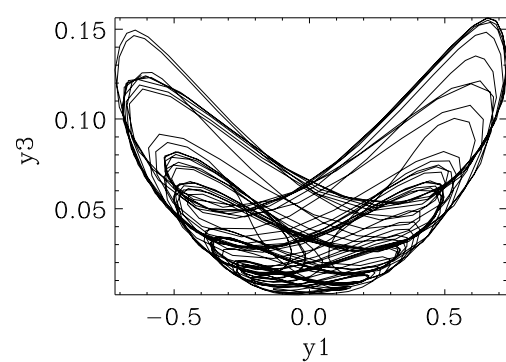

(b) $y_{1}$ gegen $y_{3}$

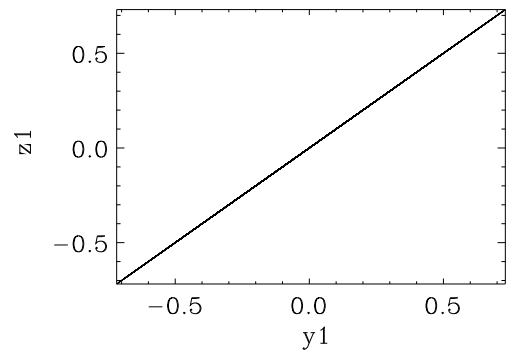

(d) $y_{1}$ gegen $z_{1}$

Abbildung 2.5.: Verallgemeinerte Synchronisation eines Chua-Oszillators welcher das Lorenzsystem treibt Glg. (2.13)-(2.14).

sucht werden. Der Chua-Schwingkreis läßt sich durch das normierte Gleichungssystem

$$
\begin{aligned}
\dot{x}_{1} & =\alpha\left(x_{2}-x_{1}-f\left(x_{1}\right)\right) \\
\dot{x}_{2} & =x_{1}-x_{2}+x_{3} \\
\dot{x}_{3} & =-\beta x_{2}-\gamma x_{3} \\
f\left(x_{1}\right) & =m_{1} x_{1}+\frac{1}{2}\left(m_{0}-m_{1}\right)\left[\left|x_{1}+1\right|-\left|x_{1}-1\right|\right]
\end{aligned}
$$

beschreiben, wobei $f\left(x_{1}\right)$ eine stückweise lineare Kennlinie der Diode $N_{R}$ modelliert. Mit den Parametern $\alpha=11.8, \beta=14.9, \gamma=0.2977, m_{0}=-1.34, m_{1}=-0.717$ zeigt das System (2.13) den chaotischen sogenannten „double scroll“ Attraktor in Abb. 2.5a. Mit 
dem Kopplungssignal $s=x_{2}$ wird nun das Lorenzsystem, wobei die $y_{2}$-Variable durch $s(t)$ ersetzt wird, getrieben

$$
\begin{aligned}
\dot{y_{1}} & =-10\left(y_{1}-s(t)\right) \\
\dot{y_{2}} & =28 y_{1}-y_{2}-y_{1} y_{3} \\
\dot{y_{3}} & =s(t) y_{1}-\frac{8}{3} y_{3} .
\end{aligned}
$$

Es muß nun gezeigt werden, daß eine identische Kopie $\mathbf{z}$ von Glg. (2.14) mit diesen System, unabhängig vom antreibenden Signal $s(t)$, identisch synchronisiert. Mit der Lyapunovfunktion $L=\frac{\left(e_{1}^{2}+e_{3}^{2}\right)}{2}$ kann man leicht zeigen, daß das $\left(e_{1}-e_{3}\right)$-Subsystem von $\mathbf{e}=\mathbf{z}-\mathbf{y}$ für alle Anfangsbedingungen und alle Kopplungssignale $s(t)$ einen asymptotisch stabilen Fixpunkt in $\mathbf{0}$ besitzt, so daß $e_{1}, e_{3}$ für $t \rightarrow \infty$ gegen Null konvergieren. Damit reduziert sich die Gleichung für $e_{2}$ auf $\dot{e_{2}}=-e_{2}$, womit $e_{2}$ auch auf den Fixpunkt 0 zuläuft. Damit ist gezeigt, daß das getriebene Lorenzsystem aus Glg. (2.14) für beliebige antreibende Signale $s(t)$ asymptotisch stabil ist und verallgemeinerte Synchronisation auftritt. In Abb. 2.5 ist dies Beispiel numerisch simuliert worden. Aufgrund der einseitigen Kopplung ist in Abb. 2.5a der „double scroll“ Attraktor des treibenden Systems zu sehen. Der Attraktor des getriebenen Lorenzsystems ist in Abb. 2.5b geplottet, und stellt sozusagen eine nichtlineare Abbildung des Attraktors aus Abb. 2.5a dar. Die Auftragung von $x_{3}$ gegen $y_{3}$ in Abb. 2.5c zeigt deutlich, daß die Systeme nicht identisch synchronisiert sind. Dagegen zeigt die Auftragung von $y_{1}$ gegen $z_{1}$, wobei $z_{1}$ von einer identischen Kopie von Glg. (2.14) stammt, in Abb. 2.5d eine Diagonale. Die identische Synchronisation mit dem "auxiliary“ System zeigt nochmals, daß das Lorenzsystem (2.14) vom Chuaoszillator versklavt wurde und GS auftritt.

\subsubsection{Eindeutigkeit der Abbildung}

Oben sind wir bei verallgemeinerter Synchronisation von einer Funktion $\mathbf{y}=\mathbf{H}(\mathbf{x})$ ausgegangen, wo jedem Zustand $\mathbf{x}$ des treibenden Systems durch $\mathbf{H}$ genau ein Zustand des getriebenen Systems y zugeordnet wird. Dies hat sich als zu starke Forderung herausgestellt [89], was man sich am Beispiel von zwei periodischen Oszillatoren klarmachen kann. Die Oszillatoren seien unidirektional gekoppelt, wobei die Perioden im Verhältnis $1: 2$ eingerastet sind, so daß das treibende $x$-System zwei Umläufe für einen Umlauf des getriebenen $y$-System benötigt. Offensichtlich entsprechen jedem Punkt des treibenden Systems zwei Punkte im getriebenen System, so daß keine eindeutige Funktion $H$ existiert, siehe Abb. 2.6. Andererseits sind die Systeme asymptotisch stabil miteinander synchronisiert $^{15}$, so daß eine feste Beziehung zwischen den Flüssen bestehen muß. Eine Definition von verallgemeinerter Synchronisation sollte dieses einfache Beispiel beinhalten um diese Bezeichnung halbwegs verdient zu haben. Im gemeinsamen Phasenraum

\footnotetext{
${ }^{15} \mathrm{Im}$ Fall von $1: p$ Einrastung existieren $p$ verschiedene Bassins gegenüber denen das getriebene System asymptotisch stabil ist. Für zwei Anfangsbedingungen aus verschiedenen Bassins befinden sich aber die Trajektorien $\mathbf{y}_{1}(t)$ und $\mathbf{y}_{2}(t)$ bei gleichem $\mathbf{x}(t)$ schließlich auf zwei verschiedenen Umläufen im getriebenen System.
} 


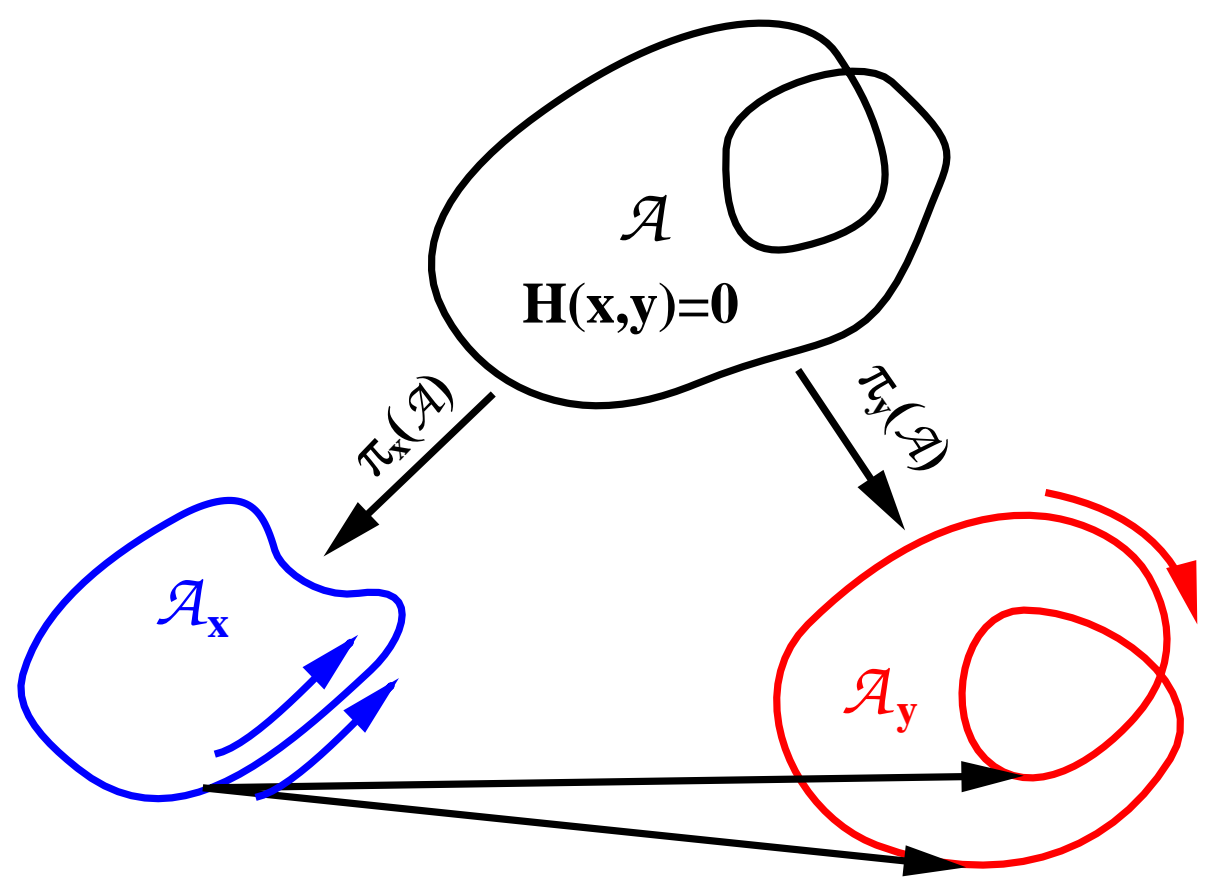

Abbildung 2.6.: 1 : 2-Einrastung von (instabilen) periodischen Orbits, wobei das treibende $\mathbf{x}$-System zwei Umläufe bei einem Umlauf vom getriebenen $\mathbf{y}$-System benötigt, was durch die Pfeile angedeutet ist.

auf dem Attraktor $\mathcal{A}$ ist die Eindeutigkeit aber wieder hergestellt, so daß man im Allgemeinen die Transformation $\mathbf{H}$ implizit definieren muß.

Im weiteren heißen zwei Systeme

$$
\begin{aligned}
\dot{\mathbf{x}} & =\mathbf{f}_{\mathbf{x}}(\mathbf{x}) \\
\dot{\mathbf{y}} & =\mathbf{f}_{\mathbf{y}}+\mathbf{g}(\mathbf{y}, \mathbf{x})
\end{aligned}
$$

$\left(\mathbf{x} \in \mathbb{R}^{n}\right.$ und $\left.\mathbf{y} \in \mathbb{R}^{m}\right)$ miteinander verallgemeinert synchronisiert, falls ein offenes Bassin $\mathcal{B} \subset \mathbb{R}^{m} \times \mathbb{R}^{n}$ existiert, welches eine kompakte invariante und attraktive Mannigfaltigkeit der Form $\mathbf{H}(\mathbf{x}, \mathbf{y})=0$ beinhaltet, so daß

$\lim _{t \rightarrow \infty}\left\|\mathbf{H}\left(\mathbf{x}_{0}(t), \mathbf{y}_{0}(t)\right)\right\|=0, \forall\left(\mathbf{x}_{0}, \mathbf{y}_{0}\right) \in \mathcal{B}$.

Subharmonische Einrastverhältnisse $T_{x}: T_{y}=1: p$ mit $p \geq 2$ können bei periodischen Systemen häufig beobachtet werden. Dieser Fall kann aber auch direkt auf chaotische Systeme übertragen werden, falls man die Synchronisation von UPOs im Attraktor untersucht [89]. Im folgenden geben wir zwei Beispiele von subharmonischer Einrastung und der damit verbundenen nicht eindeutigen Abbildung von $\mathbf{x}$ nach $\mathbf{y}$ für iterierte Abbildungen und ODEs. Als erstes betrachten wir die verallgemeinerte Bäckerabbildung 
2. Varianten der Synchronisation gekoppelter (chaot.) dynamischer Systeme

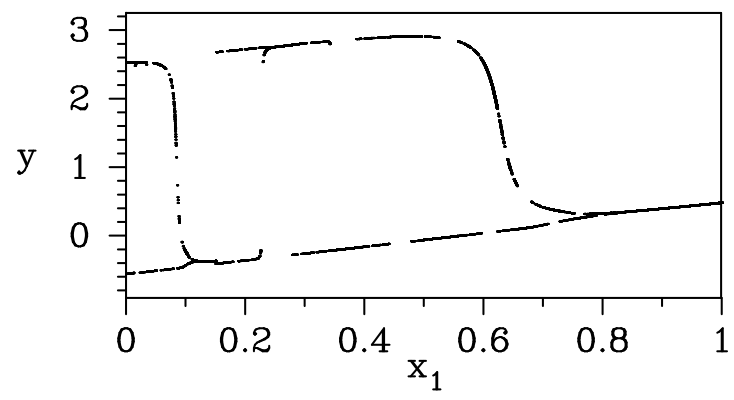

Abbildung 2.7.: Abhängigkeit des Zustandes vom getriebenen System y (2.17) von der $x_{1}$ Variable des treibenden Systems (2.16).

[82, S. $75 \mathrm{ff}]$

$$
\begin{aligned}
& x_{1}^{n+1}=f_{1}\left(x_{1}^{n}, x_{2}^{n}\right)= \begin{cases}\alpha x_{1}^{n} & \text { if } x_{2}^{n}<a_{1} \\
\alpha+\beta x_{1}^{n} & \text { if } x_{2}^{n} \geq a_{1}\end{cases} \\
& x_{2}^{n+1}=f_{2}\left(x_{1}^{n}, x_{2}^{n}\right)= \begin{cases}x_{2}^{n} / a_{1} & \text { if } x_{2}^{n}<a_{1} \\
\left(x_{2}^{n}-a_{1}\right) / a_{2} & \text { if } x_{2}^{n} \geq a_{1},\end{cases}
\end{aligned}
$$

mit $0<a_{1}, a_{2}, \alpha, \beta \in \mathbb{R}$, wobei $\alpha<\beta$ und $\alpha+\beta=a_{1}+a_{2}=1$. Die $x_{1}$ Koordinate von Gleichung (2.16) treibt das eindimensionale System

$$
y^{n+1}=\arctan \left(-c * y^{n}\right)+x_{1}^{n+1}+d .
$$

Die Parameter vom treibenden System sind $\alpha=0.15, \beta=1-\alpha, a_{1}=0.1, b_{1}=1-a_{1}$, und für das getriebene System wurden $c=40$ und $d=1$ benutzt. Die Mehrdeutigkeit der Abbildung $\mathbf{x}^{n}=\left(x_{1}^{n}, x_{2}^{n}\right) \mapsto y^{n}$ zwischen dem treibenden und getriebenen System wird in Abb. 2.7 illustriert, wo $y^{n}$ gegen $x_{1}^{n}$ aufgetragen ist ${ }^{16}$. Man erkennt sofort, daß der Graph von $\left(x_{1}, y\right)$ im wesentlichen aus zwei Zweigen besteht, was die Eindeutigkeit der Abbildung in einem großen Teil des Phasenraumes zerstört. Um dieses Verhalten besser zu verstehen, haben wir die Einrasteigenschaften von niederperiodischen UPOs des treibenden Systems untersucht. Die verallgemeinerte Bäckerabbildung (2.16) besitzt zwei Fixpunkte in $\mathbf{x}=(0,0)$ und $\mathbf{x}=(1,1)$. Falls der erste Fixpunkt zum Antrieb von Glg. (2.17) benutzt wird, dann besteht der Orbit des getriebenen Systems $\left\{y^{n}\right\}$ aus der alternierenden Folge $\{\ldots,-0.5609,2.5262,-0.5609,2.5262, \ldots\}$, während die Antwort auf den zweiten Fixpunkt $(1,1)$ mit $y^{n} \approx 0.4811$ konstant ist. Damit wird ein Fixpunkt auf einen Fixpunkt abgebildet, während der andere mit einem Periode-2 Orbit beantwortet wird. Für die Periode-2 Orbits von Glg. (2.16) beobachten wir eine andere Situation.

$$
\begin{aligned}
\mathbf{x}^{n} & =\left(\frac{\alpha}{1-\alpha \beta}, \frac{a_{1}^{2}}{1-a_{1} b_{1}}\right) \approx(0.171920,0.0109890) \\
\mathbf{x}^{n+1} & =\left(\frac{\alpha^{2}}{1-\alpha \beta}, \frac{a_{1}}{1-a_{1} b_{1}}\right) \approx(0.025788,0.109890)
\end{aligned}
$$

\footnotetext{
${ }^{16}$ Bei der Bäckerabbildung ist es ausreichend $y^{n}$ nur gegen $x_{1}^{n}$ aufzutragen, da die Invertierung von $x_{1}^{n+1}$ unabhängig von $x_{2}^{n}$ ist [82].
} 


\section{Varianten der Synchronisation gekoppelter (chaot.) dynamischer Systeme}

Es wird zwar 1 : 1-Einrastung beobachtet, aber je nach Anfangsbedingung ( $y<y_{b}$ bzw. $\left.y>y_{b}, y_{b} \approx 0.05141\right)$ von $y^{n}$, antwortet das getriebene System mit zwei verschiedenen Periode-2 Orbits; $\mathrm{x}^{n} \mapsto y^{n} \approx 2.69609, \mathrm{x}^{n+1} \mapsto y^{n+1} \approx-0.535736$ für $y<y_{b}$ und $\mathbf{x}^{n} \mapsto y^{n} \approx-0.389005, \mathbf{x}^{n+1} \mapsto y^{n+1} \approx 2.53241$ für $y>y_{b}$. In beiden Fällen ist die Abbildung von treibenden Periode-2 Orbit zum jeweiligen Orbit des getriebenen Systems eindeutig. Die beiden Bassins sind aber nur für diesen Orbit relevant und haben keinen weiteren Einfluß auf GS ( i.e. zwei Trajektorien von $y$, welche in den beiden verschieden Bassins starten, konvergieren bei Antrieb mit einer chaotischen Lösung aus Glg. (2.16) schließlich auf die gleiche Trajektorie ). Beide Periode-3 UPOs von Glg. (2.16) erzeugen Periode-6 Orbits im getriebenen System und die Abbildung kann wiederum nur sauber implizit $\mathbf{H}(\mathbf{x}, y)=0$ definiert werden. Dieses Verhalten setzt sich bei höherperiodischen UPOs immer weiter fort, welches dann auch die mehrdeutige Abhängigkeit $y^{n}$ von $x_{1}^{n}$ (bzw. $\mathbf{x}^{n}$ ) in Abb. 2.7 erklären kann.

Ähnliche subharmonische Einrastverhältnisse können auch bei Systemen mit kontinuierlicher Zeit beobachet werden. Als Beispiel betrachten wir zwei unidirektional gekoppelte Rösslersysteme:

$$
\begin{array}{ll}
\dot{x}_{1}=2+x_{1}\left(x_{2}-4\right) & \alpha \dot{y}_{1}=2+y_{1}\left(y_{2}-4\right) \\
\dot{x}_{2}=-x_{1}-x_{3} & \alpha \dot{y}_{2}=-y_{1}-y_{3} \\
\dot{x}_{3}=x_{2}+b x_{3} & \alpha \dot{y}_{3}=y_{2}+b y_{3}+c\left(x_{3}-y_{3}\right)
\end{array}
$$

Der Parameter $\alpha=2$ sorgt für eine Verstimmung der mit variabler Kopplungsstärke $c$ gekoppelten Systeme. Abbildung 2.8a zeigt die Projektion des treibenden Rösslerattraktors (graue durchgezogene Linie) zusammen mit zwei niederperiodischen UPOs (schwarze durchgezogene und gestrichelte Linie), welche eingebettet im chaotischen Attraktor sind. In Abb. 2.8b sind die korrespondierenden chaotischen und periodischen Orbits des getriebenen Systems gezeigt. Bei der gewählten Verstimmung der Systeme von $\alpha=2$ ist identische Synchronisation nicht mehr möglich. Für die Kopplungsstärke $c=0.27$ ist der größte bedingte Lyapunovexponent $\lambda_{C}$ negativ, so daß das getriebene System im Mittel stabil ist. Bei diesem Wert treten aber auch verschiedene Einrastverhältnisse bei unterschiedlichen UPOs auf. Der UPO, welcher mit durchgezogener Linie in Abb. 2.8a gezeigt ist, synchronisiert mit einem periodischen Orbit gleicher Periode des getriebenen Systems (siehe Abb. 2.8c). Falls der andere UPO (gestrichelte Linie in Abb. 2.8a) als Antrieb benutzt wird, antwortet das getriebene System mit einem Orbit doppelter Periode (siehe Abb. 2.8d), weswegen in diesem Fall wieder jeder Punkt des treibenden UPOs auf je zwei Punkte des Orbits im getriebenen System abgebildet wird. Trotz dieser 1 : 2-Einrastung ist das getriebene System asymptotisch stabil, welches mit einem „auxiliary"-System [2] nachgewiesen werden konnte (siehe Abb. 2.8e, wo exemplarisch die $y_{1}$-Komponente von zwei identischen Kopien aufgetragen ist). Der Parameter $b$ von den Hilfssystemen wurde mit $b=0.422$ und $b=0.418$ leicht gegenüber dem treibenden System $b=0.42$ aus Glg. (2.18) verstimmt, wodurch gleichzeitig die Robustheit von GS getestet werden konnte. Aufgrund des kleinen Parameterunterschiedes zeigt Abb. 2.8e keine perfekte Gerade, wobei sich aber die Trajektorien im Beobachtungszeitraum nur 

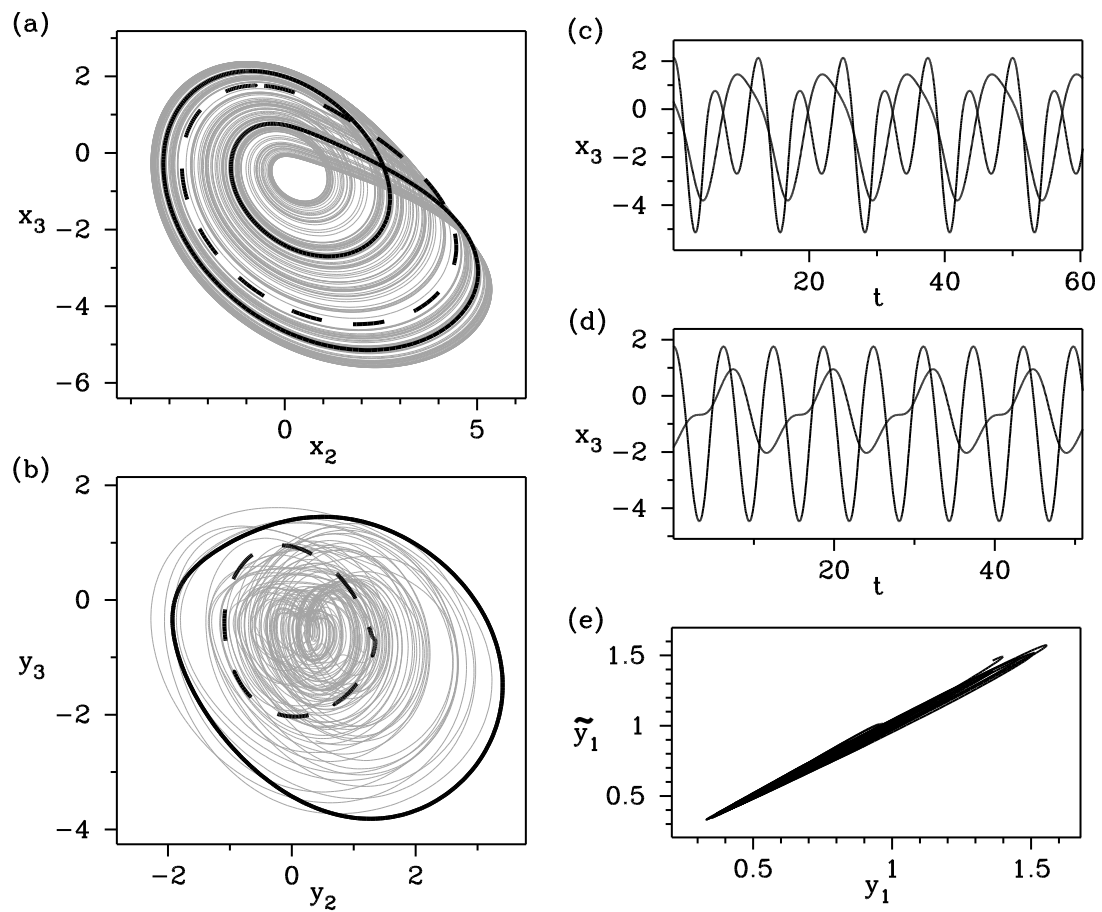

Abbildung 2.8.: Unidirektional gekoppelte Rössler Systeme (2.18) für $c=0.27$ und $b=0.42$. (a) Chaotischer Attraktor (durchgezogene graue Kurve) und zwei UPOs (durchgezogene und gestrichelte schwarze Linien); (b) Attraktor (grau) und periodische Orbits (PO) (durchgezogene und gestrichelte schwarze Linien), welche mit denen in (a) synchronisiert sind; (c) Periodische Oszillation des ersten UPO mit seinem korrespondierenden periodischen Orbit $(1: 1)$; (d) wie in (c), aber für das zweite UPO-PO Paar $(1: 2)$; (e) $y_{1}$ Variablen von zwei leicht unterschiedlichen getriebenen Systemen (,auxiliary“-System).

unwesentlich von ihr entfernen, so daß transversale Instabilitäten weitgehend ausgeschlossen werden können. Die Ergebnisse bleiben auch bei der Addition von schwachem Rauschen qualitativ unverändert.

Falls die Kopplungsstärke $c$ erhöht wird, synchronisieren alle untersuchten UPOs mit gleichem Einrastverhältnis $T_{x}=T_{y}$, wobei aber immer noch Regionen im chaotischen Attraktor existieren, wo Nächste-Nachbarn eines Referenzzustandes auf verschiedene Zweige des Attraktors des getriebenen Systems abgebildet werden (siehe Abb. 2.9). Die Abbildung wurde folgendermaßen generiert: (i) man nehme zur Zeit $t$ den Zustand $\mathbf{x}(t)$ des Treibers und seine 10 nächsten Nachbarn, (ii) berechne den Abstand $d$ der korrespondierenden Zustände im getriebenen System zu y $(t)$ und (iii) trage den Abstand $d$ gegen die Zeit $t$ auf. Anhand Abb. 2.9 wird deutlich, daß entlang einer typischen Trajektorie mehrere Regionen existieren, wo der Zustand $\mathbf{x}(t)$ auf mehrere Bereiche im getriebenen System abgebildet wird. Da alle von uns untersuchten niederperiodischen UPOs $1: 1$ 


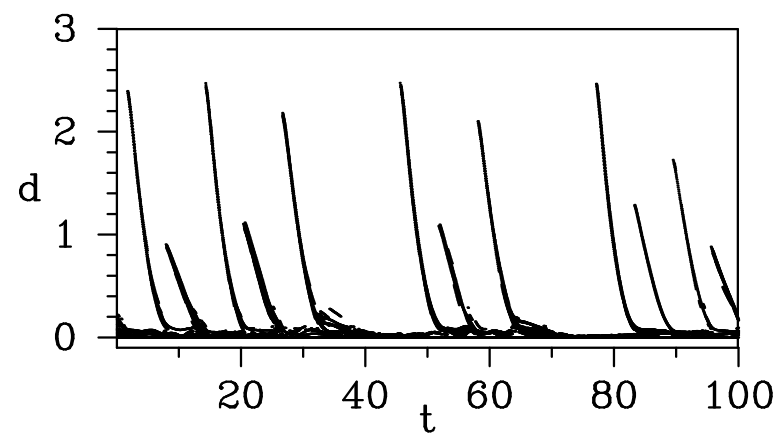

Abbildung 2.9.: Abstände $d$ von Zuständen des getriebenen System, welche den 10 Nächsten-Nachbarn des treibenden Systems $(c=2, b=0.42)$ entsprechen.

einrasten und subharmonische Einrastung in diesem Kopplungsbereich bezüglich des UPO-Maßes nicht beobachtet wurde, muß noch eine andere Möglichkeit für die beobachtete Nichteindeutigkeit der Abbildung von $\mathbf{x} \mapsto \mathbf{y}$ existieren. Im nächsten Abschnitt wird gezeigt, daß die Abbildung von $\mathbf{y}=\mathbf{H}(\mathbf{x})$ zwar eindeutig, aber auch fraktal sein kann, welches solch ein Verhalten auch in Abwesenheit von subharmonischen Einrastungen erklären kann. Eine Bedingung für eine glatte Abbildung ist, daß der größte Lyapunovexponenten vom getriebenen System kleiner als der kleinste des Treibers ist [47], welche in diesem Fall $-0.44=\lambda_{1}^{y}>\lambda_{3}^{x}=-3.2$ deutlich verletzt ist und somit ein Hinweis auf eine möglicherweise eindeutige aber fraktale Abbildung ist.

\subsubsection{Glattheit der Abbildung}

In den letzten beiden Abschnitten haben wir uns mit der Existenz und Eindeutigkeit der Abbildung $\mathbf{y}=\mathbf{H}(\mathbf{x})$ beschäftigt, wobei herauskam, daß im allgemeinsten Fall die Funktion $\mathbf{H}$ und damit die invariante Synchronisationsmannigfaltigkeit $\mathcal{M}_{\text {Synch }}$ eigentlich nur implizit durch $\mathbf{H}(\mathbf{x}, \mathbf{y})=0$ definiert werden kann. Ob die Mannigfaltigkeit darüberhinaus in jedem Punkt $\mathbf{z}=(\mathbf{x}, \mathbf{y})$ glatt, also stetig und differenzierbar, ist, kann mit den bisherigen Methoden nicht nachgewiesen werden. Ein Indiz ist, daß die Dimension des Attraktors $\mathcal{A}$ im gemeinsamen Phasenraum $\mathbb{R}^{n} \times \mathbb{R}^{m}$ identisch zur der des Attraktors $\mathcal{A}_{x} \subset \mathbb{R}^{n}$ des treibenden Systems ist, d.h. $\operatorname{dim}\{\mathcal{A}\} \equiv \operatorname{dim}\left\{\mathcal{A}_{x}\right\}$ [108], da fraktale Abbildungen zu einer Erhöhung der Dimension führen. Zum Nachweis der Glattheit von $\mathcal{M}_{\text {Synch }}$ bedienen wir uns der Theorie invarianter Mannigfaltigkeit, wobei wir im wesentlichen der Argumentation aus [60] folgen werden. Im weiteren nehmen wir an, daß $\mathcal{M}_{\text {Synch }}$ existiert, so daß der gemeinsame Phasenraum $\mathbb{R}^{n} \times \mathbb{R}^{m}$ in zwei orthogonale Unterräume aufgeteilt werden kann, wobei $N_{z} \mathcal{M}$ transversal und $T_{z} \mathcal{M}$ parallel zu einem Punkt $\mathbf{z}=(\mathbf{x}, \mathbf{y}) \in \mathcal{M}_{\text {synch }}$ sind. Die invariante Mannigfaltigkeit $\mathcal{M}_{\text {synch }}$ ist stabil, falls in jedem Punkt $\mathbf{z} \in \mathcal{M}_{\text {synch }}$ mit $\mathbf{z}_{\perp}(0) \in N_{z} \mathcal{M}$

$$
\lim _{t \rightarrow \infty}\left\|\mathbf{z}_{\perp}(t)\right\|=0
$$


gilt. Diese Definition entspricht offensichtlich der schon oft benutzten Eigenschaft der asymptotischen Stabilität von $\mathcal{M}_{\text {Synch }}$ und ist notwendig für das Auftreten von jeglicher Art von funktionalen Zusammenhängen zwischen den Flüssen der gekoppelten Systeme. Eine invariante Mannigfaltigkeit $\mathcal{M}_{\text {synch }}$ ist normal hyperbolisch, falls in jedem Punkt $\mathbf{z} \in \mathcal{M}_{\text {synch }}$ mit $\mathbf{z}_{\perp}(0) \in N_{z} \mathcal{M}$ und $\mathbf{z}_{\|}(0) \in T_{z} \mathcal{M}$

$$
\lim _{t \rightarrow \infty} \frac{\left\|\mathbf{z}_{\perp}(t)\right\|}{\left\|\mathbf{z}_{\|}(t)\right\|}=0
$$

gilt. In anderen Worten muß die transversale Kontraktion zu $\mathcal{M}_{\text {synch }}$ stärker als die tangentiale innerhalb von $\mathcal{M}_{\text {synch }}$ sein. Normale Hyperbolizität sichert die Glattheit und darüberhinaus noch die Robustheit von $\mathcal{M}_{\text {synch }}$ gegenüber kleinen Störungen des Systems, wie z.B. Parameterunterschiede oder Rauschen [137].

Die Stabilitätsbedingungen können auch in Form von transversalen und tangentialen Lyapunovexponenten ausgedrückt werden. Für ein ergodisches invariantes Maß in $\mathcal{A} \subset$ $\mathcal{M}_{\text {synch }}$ existieren im System (2.15) $n$ tangentiale $\lambda_{\|}$und $m$ transversale Lyapunovexponenten $\lambda_{\perp}$. Sei nun $\lambda_{\|}^{\text {min }}$ der kleinste tangentiale und $\lambda_{\perp}^{\max }$ der größte transversale Lyapunovexponent bezüglich aller existierenden Maße in $\mathcal{A}$, so ist die invariante Mannigfaltigkeit $\mathcal{M}_{\text {synch }}$ stabil, falls $\lambda_{\perp}^{\max }<0$ ist. $\mathcal{M}_{\text {synch }}$ ist normal hyperbolisch falls darüberhinaus

$$
\lambda_{\perp}^{\max }<\lambda_{\|}^{\min }
$$

gilt $^{17}$. Das Problem ist nun wieder, daß die Bedingung (2.21) bezüglich aller invarianten Maße auf $\mathcal{A}$ erfüllt sein muß, um eine hinreichende Aussage zu erhalten. Dafür hat man nun eine mathematisch exakte Aussage erhalten, welche für das grundsätzliche Verständnis von GS wesentlich ist.

Nun betrachten wir ein Beispiel [60, 90], wo der Übergang von einer fraktalen zu einer glatten Abbildung analytisch untersucht werden kann. Dazu verwenden wir wieder die verallgemeinerte Bäckerabbildung aus Glg. (2.16). Das natürliche Maß des Attraktors von Glg. (2.16) ist gleichverteilt in $x_{2}$ und variert sehr wild in der $x_{1}$-Komponente. Die Lyapunovexponenten bezüglich des natürlichen Maßes sind $\lambda_{\|}^{1}=a_{1} \ln \left(1 / a_{1}\right)+$ $a_{2} \ln \left(1 / a_{2}\right)>0$ und $\lambda_{\|}^{2}=a_{1} \ln (\alpha)+a_{2} \ln (\beta)<0$. Zusätzlich gilt für alle invarianten Maße die Ungleichung $\ln (\alpha) \leq \lambda_{\|}^{2} \leq \ln (\beta)$, so daß der kleinste Lyapunovexponent durch $\lambda_{\|}^{\min }=\ln (\alpha)$ und der größte durch $\lambda_{\|}^{\max }=\ln (\beta)$ eindeutig bestimmt sind. Die Bäckerabbildung (2.16) treibt nun das lineare System

$$
y^{n+1}=a y^{n}+\varepsilon \cos \left[2 \pi x_{1}^{n}\right],
$$

mit $\varepsilon>0$ als Kopplungsparameter und $|a|<1$.

\footnotetext{
${ }^{17}$ Diese Bedingung wurde von der Idee her im Prinzip schon in [47] und [108] vorgeschlagen, wobei dort mehr oder weniger heuristisch argumentiert wurde. Wir wollen hier der Arbeit [60] folgen, wo der Bezug zur Theorie der invarianten Mannigfaltigkeiten explizit herausgearbeitet wurde und dadurch mathematisch exakte Aussagen getroffen werden können.
} 
Die neue Synchronisationsmannigfaltigkeit kann analytisch durch eine Funktion

$$
y=\varepsilon \sum_{j=1}^{\infty} a^{j-1} \cos \left[2 \pi f_{1}^{-j}\left(x_{1}, x_{2}\right)\right]=\varepsilon \sum_{j=1}^{\infty} g_{j}\left(x_{1}, x_{2}\right)
$$

der Zustandsvariablen $x_{1}^{n}$ und $x_{2}^{n}$ vom treibendem System angegeben werden. Für $|a|<1$ sind die Funktionen $g_{j}$ beschränkt und stetig, so daß die Summe aus Glg. (2.23) wohldefiniert ist und gleichmäßig gegen eine stetige Grenzfunktion konvergiert. Diese Grenzfunktion definiert eine global attraktive invariante Manngifaltigkeit des gekoppelten Systems aus Glg. (2.16) und Glg. (2.22). Bei der Bäckerabbildung (2.16) hängt die Inverse $f_{1}^{-j}$ nur von $x_{1} \mathrm{ab}$, so daß die Funktion (2.23) unabhängig von $x_{2}$ ist. Der Lyapunovexponent transversal zu Glg. (2.23) ist gegeben durch $\ln a$ und die tangentialen sind die der Bäckerabbildung. Aufgrund der gleichmässigen Konvergenz der Reihe aus Glg. (2.23) für $|a|<1$ ist die Stetigkeit der Funktion $y=H\left(x_{1}\right)$ gesichert. Die Frage der Differenzierbarkeit kann in diesem Beispiel auch analytisch beantwortet werden. Mit Glg. (2.16) kann Glg. (2.23) umgeschrieben werden zu

$$
y=\varepsilon \sum_{j=1}^{\infty} a^{j-1} \cos \left[2 \pi\left(\alpha^{-j_{1}} \beta^{-j_{2}} x_{1}\right)+c_{j}\right],
$$

mit $j_{1}+j_{2}=j$ und $c_{j} \in \mathbb{R}$. Für den Fall $b=\alpha=\beta$ ergibt sich die Reihenentwicklung der Weierstraßfunktion

$$
y=\frac{\varepsilon}{a} \sum_{j=1}^{\infty} a^{j} \cos \left[2 \pi\left(b^{-j} x_{1}\right)+c\right]
$$

Für $a<b$ gibt

$$
\frac{\partial y}{\partial x}=-\frac{2 \pi \varepsilon}{a} \sum_{j=1}^{\infty}\left(\frac{a}{b}\right)^{j} \sin \left[2 \pi\left(b^{j} x_{1}\right)+c\right]
$$

die Ableitung der Weierstraßfunktion. Für $a>b$ divergiert die Summe und man kann zeigen, daß Glg. (2.24) in keinem Punkt differenzierbar ist, aber aufgrund der gleichmässigen Konvergenz überall stetig. Für $\alpha=\beta$ existiert somit am kritischen Punkt $a=b$ ein scharfer Übergang von einer glatten $(a<b)$ zu einer fraktalen $(a>b)$ Mannigfaltigkeit bzw. Abbildung von $x \mapsto y$.

Die bei GS auftretende komplizierte Synchronisationsmannigfaltigkeit wird wohl nur in wenigen weiteren Systemen einer analytischen Analyse zugänglich sein, so daß man in den meisten Fällen wieder auf Lyapunovexponenten (bezüglich des natürlichen Maßes) zurückgreifen wird. Ein anderer Ansatz, welcher auch in Experimenten leichter anwendbar sein wird, ist aus Zeitreihen direkt die Abbildung $\mathbf{H}$ zu approximieren [16] bzw. sie auf Stetigkeit und Differenzierbarkeit zu untersuchen [114]. Damit können zwar keine exakten Resultate erhalten werden, aber dafür kann auch bei unbekannten Systemgleichungen Evidenz für GS nachgewiesen werden. 


\subsection{Frequenz- und Phasensynchronisation}

Bis jetzt wurden sehr starke Synchronisationsvarianten vorgestellt, wo die Flüsse der gekoppelten Systeme in jedem Punkt miteinander fest verknüpft waren. Man kann aber auch die Synchronisation zwischen globalen Eigenschaften, wie z.B. die mittlere Frequenz, der Systeme betrachten. In diesen Fällen kann das getriebene System einen Großteil seiner Freiheitsgrade behalten, welches dann oft auch als partielle Synchronisation bezeichnet wird. Die Phasen- und Frequenzsynchronisation kann man als direkte Verallgemeinerung der klassischen Synchronisationsphänomene á la Huygens ansehen. Synchronisation periodischer selbsterregter Oszillatoren, die ein- oder beidseitig miteinander gekoppelt sind, wird klassisch durch das Auftreten einer festen Phasendifferenz $\phi_{1,2}=m \phi_{1}-n \phi_{2}, m, n \in \mathbb{N}$ für alle Zeiten $t$ definiert. Diese Definition beinhaltet damit auch nichtlineare Phänomene wie Erzeugung von höheren harmonischen oder auch subharmonischen Schwingungen [134] und fordert insbesondere nicht die Gleichheit der beiden Systeme. Die Konstruktion von einer Phasenvariable $\phi$ ist bei periodischen Oszillatoren offensichtlich, und eröffnet ein breites Feld von Untersuchungsmöglichkeiten solcher Systeme. Bei chaotisch schwingenden Oszillatoren aber, die per Definition ein unendlich ausgedehntes Spektrum haben, scheint auf den ersten Blick die Einführung einer Phasenvariable keinen Sinn zu machen. Die Theorie der Signalverarbeitung liefert jedoch im Prinzip die Möglichkeit jedes Signal $s(t)$ zu einem festen Zeitpunkt in Betrag und Phase zu zerlegen, indem man mit Hilfe der Hilberttransformation $\tilde{s}(t)$ von $s(t)$ das sogenannte analytische Signal

$$
\psi(t)=s(t)+i \tilde{s}(t)=A(t) e^{i \phi(t)}
$$

konstruieren kann [84]. Man kann sich das analytische Signal als Ausgangsignal eines Filters vorstellen, dessen Impulsantwort die Amplituden unverändert läßt, aber jeder Frequenz eine konstante Phasenverschiebung von $\frac{\pi}{2}$ aufprägt. Mit dieser Methode kann man zu jedem Signal formal eine instantane Phasenvariable $\phi(t)$ definieren ${ }^{18}$. Ob die gewonnene Phase auch physikalisch sinnvoll ist (i.e. monoton steigt), ist eine andere Frage und es existieren einige Beispiel wo die Phase aus dem analytischen Signal unphysikalisch ist $^{19}$. Andererseits hängt die Phase eines Oszillators mit dem Null Lyapunovexponenten zusammen, so daß im Prinzip auch für jedes chaotische System ein vernünftige phasenartige Größe existieren sollte.

Bei chaotische Oszillatoren mit einer ausgeprägten mittleren Umlauffrequenz kann man oft die Phasendynamik als schwach gekoppelt mit der Amplitudendynamik ansehen

$$
\frac{\partial \phi}{\partial t}=\omega+c \Psi(t), c \ll 1 .
$$

\footnotetext{
${ }^{18}$ Das Konzept des analytischen Signals scheitert aber bei Systemen bei denen die Amplitude $A(t)$ von $\psi(t)$ Nulldurchgänge besitzt, da in diesem Punkt keine Phase definiert ist und diese dann beliebige Werte annehmen kann. In diesem Fall wird man versuchen in ein Koordinatensystem zu wechseln, wo solch ein Nulldurchgang nicht vorkommt.

${ }^{19}$ Eine Verallgemeinerung der Hilberttransformation, welche für beliebige Signale physikalisch sinnvolle Phasen liefert wurde in [43] vorgeschlagen. Es existieren aber noch einige Probleme hinsichtlich des Algorithmus und der Interpretation der erhaltenen Phasen, so daß zur Zeit noch offen ist, ob hiermit für beliebige Signale eine physikalisch interpretierbare Phase definiert werden kann.
} 
Koppelt man nun zwei Oszillatoren aus Glg. (2.26) mit mittleren Eigenfrequenzen $\omega_{1}$ und $\omega_{2}$, so läßt sich die Dynamik der Phasendifferenz $\Delta \phi=n \phi_{1}-m \phi_{2}$ durch

$$
\frac{\partial \Delta \phi}{\partial t}=n \omega_{1}-m \omega_{2}+\varepsilon G(\Delta \phi)+c \Psi(t), c \ll 1
$$

modellieren. Man erhält dann im wesentlichen eine mit Stärke $\varepsilon$ durch $G(\Delta \phi)$ gekoppelte Phasendynamik, welche schwach durch die Amplitudendynamik $c \Psi(t)$ gestört wird. Solche Systeme verhalten sich damit wie verrauschte periodische Oszillatoren, so daß eine im Mittel konstante Phasendifferenz auch im chaotischen Fall möglich erscheint.

\section{Definition 2.2 :}

Zwei dynamische Systeme besitzen die Eigenschaft der Phasensynchronisation (PS) [79, 92, 111, 110], falls zwei Zahlen $m, n \in \mathbb{N}$ existieren, so daß nach einer Transiente $0<t \leq t_{0}$ die Differenz der auf $\mathbb{R}$ fortgesetzten Phasen $\phi_{1}, \phi_{2} \in \mathbb{R}$

$$
\left|m \phi_{1}-n \phi_{2}\right|<\text { konst. } \quad \forall t>t_{0}
$$

nach oben beschränkt bleibt.

Diese Definition umfaßt offensichtlich auch die der klassischen (periodischen) Synchronisation. Zum Nachweis von PS sind wir am Langzeitverhalten der Phasendifferenz interessiert, weswegen die auf $\mathbb{R}$ fortgesetzten Phasen der Einzeloszillatoren verwendet werden müssen. Bei Verwendung von zyklischen Phasen $\tilde{\phi} \in[0,2 \pi]$ können Phasensprünge, z.B. von $\pm 2 \pi$, unbeobachtet bleiben, so daß dann die Beschränktheit der Phasendifferenz nicht weiter garantiert werden kann. Falls man aber nur an PS im statistischen Sinne interessiert ist, kann auch ein Histogramm der Differenz von den zyklischen Phasen zum Nachweis verwendet werden. Für Details wird auf die Diskussion in Abschn. 4.4.1 verwiesen. Die Definition von Phasensynchronisation macht keinerlei Voraussetzungen an die Amplituden der gekoppelten Oszillatoren, so daß bei chaotischen Systemen die Amplituden weiterhin chaotisch schwingen können und miteinander völlig unkorreliert sein dürfen! Oft muß selbst bei chaotischen Signalen nicht der Weg über die Hilberttransformation gegangen werden, um eine geeignete Phasenvariable definieren zu können. Die Geometrie des Rössler-Attraktors in Abb. 2.3a erlaubt zum Beispiel die Einführung einer instantanen Phase $\phi(t)$, indem man sich in das „Loch“ in der Mitte des Attraktors setzt, um das alle Zustände herumlaufen müssen. An diesem speziellen Punkt kann man dann eine Phase durch

$$
\phi(t)=\arctan \left(\frac{x_{2}(t)}{x_{1}(t)}\right)
$$

einführen. Diese Konstruktion läßt sich praktisch immer bei Oszillatoren mit einer ausgeprägten Eigenfrequenz im Spektrum durchführen. Mit Hilfe dieser Phasenvariable läßt sich nun eine mittlere Umlauffrequenz um diesen Punkt definieren

$$
\Omega=\lim _{t \rightarrow \infty} \frac{\phi(t)}{t} .
$$


Aus der Definition der Phasensynchronisation folgt damit sofort, daß auch die mittleren Eigenfrequenzen aus Glg. (2.30) in einem rationalen Verhältnis $\Omega_{1}: \Omega_{2}=n: m$ zueinander stehen müssen, so daß Phasensynchronisation auch zu der bei periodischen Oszillatoren bekannten Frequenzeinrastung führt. Umgekehrt impliziert Frequenzsynchronisation(FS) nicht zwangsläufig Phasensynchronisation, wofür in Abschn. 4.4.1 auch ein Beispiel gegeben wird, so daß man i.A. bei Erhöhung der Kopplungsstärke $\varepsilon$ zuerst Einrastung der mittleren Frequenzen und später erst Phasensynchronisation beobachtet. Beim Übergang zu PS wird einer der beiden Null Lyapunovexponenten der gekoppelten Oszillatoren negativ, was analog zum periodischen Fall als Angleichung von Rhythmen interpretiert werden kann. Der Null Lyapunovexponent entspricht der Verschiebung entlang des Flusses, welche im Fall von PS bei beiden Systemen synchron geschieht. Bei PS und FS wird sozusagen nur ein gemeinsames „Timing“ hergestellt, welches auch die einzige Forderung an Gemeinsamkeiten beider Flüsse darstellt. Aus diesem Grund ist der Übergang zu PS bzw. FS auch mit keiner Dimensionsreduktion im gemeinsamen Phasenraum verbunden, weswegen auch die Amplituden beider Systeme weiterhin völlig unkorreliert bleiben können. Das Auftreten von PS kann, motiviert durch die Theorie zur Synchronisation schwach gekoppelten periodischer Oszillatoren [68], mittels der Einrasteigenschaften von UPOs charakterisiert und tiefer verstanden werden, wozu auf die Arbeit [104] verwiesen wird.

Kurz nach der Entdeckung von PS in numerischen Beispielen [110] konnte das Auftreten von Phasensynchronisation auch experimentell demonstriert werden [92]. PS und FS haben sich als sehr robust gegenüber Parameterunterschieden und Rauschen herausgestellt und wurden inzwischen in einer Vielzahl von komplexen Systemen nachgewiesen, z.B. numerisch in Feldern gekoppelter Differentialgleichungen [79], bei pulsgekoppelten Oszillatoren mit zeitverzögerter Kopplung [31], mit binärer Kopplung [98] und auch in partiellen Differentialgleichungen [55], worauf in Abschn. 4.4.1 noch ausführlich eingegangen wird. Als Beispiele für PS und FS in natürlichen Systemen mögen hier, die Synchronisation des 11-jährigen Sonnenaktivitätszyklus (Anzahl der Sonnenflecken) mit der Schwerpunktsbewegung der Sonne [83], der Nachweis von PS in MEG-Messungen [131] und die Synchronisation des Herzschlages mit der Atemfrequenz [118], dienen. 


\section{Die treibende Kraft}

Nach dem im letzten Abschnitt die wichtigsten Formen der chaotischen Synchronisation dynamischer Systeme, deren Nachweis und den Übergang begleitende Phänomene ausführlich behandelt wurden, soll nun der wohl wichtigste Bestandteil interagierender Systeme, die Kopplung, diskutiert werden. Die Wahl der Kopplung entscheidet über die Richtung und Stärke des Informationsflusses und damit auch darüber, welches System Einfluß nimmt (treibt) bzw. welches beinflußt (getrieben) wird. Es macht i.A. auch einen Unterschied ob uni- oder bidirektionale Kopplung vorliegt. So kann bei unidirektionaler Kopplung das getriebene System den Attraktor des treibenden Systems (IS) bzw. ein Funktion dessen (GS) nur reproduzieren, während bei bidirektionaler Kopplung auch ein neuer (synchroner) Attraktor im gekoppelten System entstehen kann, welcher in den Einzelsystemen vorher nicht existiert hat. Für das Verständnis von großen komplexen Systemen, wie z.B. neuronale Netzwerke, ist das Erkennen ob und in welcher Richtung die Untersysteme gekoppelt sind essentiell. Viele Arbeiten zur Aufklärung der Funktionsweise des Gehirn beschäftigen sich deswegen „nur" mit der Erkennung welche Teile des Kortex miteinander verbunden sind und hoffen dadurch eines Tages die zugrundeliegende Informationsverarbeitung besser zu verstehen [6, 123]. Ein anderes Beispiel sind neuronale Netze, wo die entscheidende Lernphase des Netzes ausschließlich im Auffinden der optimalen Kopplungsgewichte der einzelnen Neuronen besteht. So hat sich die Forschung zur chaotischen Synchronisation in ihren Anfängen auch hauptsächlich mit der Konstruktion von Kopplungsstrategien von „nur" zwei gekoppelten niederdimensionalen Systemen beschäftigt [93, 99, 116], welche danach auch auf komplexere Systeme, wie Felder gekoppelter iterierter Abbildungen [42, 50] oder DGL's [41, 62] und partielle Differentialgleichungen [11, 57, 64], erfolgreich verallgemeinert werden konnten. Das Problem zu einem Paar (chaotischer) Systeme sytematisch eine Kopplungsfunktion und die richtigen (möglichst skalare) Meßgrößen zu finden, welche zur identischen Synchronisation führen, ist bis heute noch Gegenstand aktueller Forschung [18]. Ein neuer Lösungsvorschlag dazu wird in Abschnitt 3.2 vorgestellt. Zuerst sollen aber im nächsten Abschnitt die wichtigsten Kopplungsstrategien, die zur Synchronisation dynamischer Systeme eingesetzt werden, diskutiert werden. 


\subsection{Wahl der Kopplung}

\subsubsection{Stabile Subsysteme}

Eine der ersten Kopplungsmethoden zur Synchronisation von chaotischen System wurde von Pecora \& Carroll [99] eingeführt. Der Ansatz beruht auf einer Zerlegung des Vektorfeldes von $\dot{\mathbf{z}}=\mathbf{f}(\mathbf{z})$ in ein stabiles $\mathbf{f}_{w}$ und ein instabiles $\mathbf{f}_{v}$ Subsystem

$$
\begin{gathered}
\dot{\mathbf{v}}=\mathbf{f}_{v}(\mathbf{v}, \mathbf{w}) \\
\dot{\mathbf{w}}=\mathbf{f}_{w}(\mathbf{v}, \mathbf{w}),
\end{gathered}
$$

mit den neuen Zuständen $\mathbf{v}=\left(z_{\tau_{1}}, \cdots, z_{\tau_{k}}\right)^{t r}$ und $\mathbf{w}=\left(z_{\tau_{k+1}}, \cdots, z_{\tau_{n}}\right)^{t r}$, wobei $\tau$ eine Permutation der Indizes $\{1, \cdots, n=\operatorname{dim}(\mathbf{f})\}$ ist. Durch diese Zerlegung entstehen zwei beidseitig gekoppelte dynamische Systeme, wobei die Anordnung so gewählt wird, daß Glg. (3.2) für $\mathbf{v} \equiv 0$ ein stabiles System ist, d.h. es besitzt einen asymptotisch stabilen Fixpunkt im Nullpunkt. Das System (3.2) besitzt keine eigenen Freiheitsgrade, weswegen eine identische Kopie von w

$$
\dot{\mathbf{w}^{\prime}}=\mathbf{f}_{w}\left(\mathbf{v}, \mathbf{w}^{\prime}\right)
$$

getrieben vom gleichem Kopplungssignal $\mathbf{v}$, mit w synchronisieren muß. Mathematisch folgt dies daraus, daß sich die transversale Dynamik $\mathbf{x}_{\perp}=\frac{1}{2}\left(\mathbf{w}-\mathbf{w}^{\prime}\right)$ auf die von $\mathbf{w}$ reduziert, d.h. die Jacobimatrizen sind identisch $\underline{J} \mathbf{x}_{\perp} \equiv \underline{J} \mathbf{w}$. Aufgrund ihrer Natur wird diese Kopplungsvariante oft auch als „master"-,,slave" Kopplung bezeichnet. Ein Nachteil ist, daß von der begrenzten Anzahl von möglichen Zerlegungen $\frac{n(n-1)}{2}$ oft nur wenige zur Synchronisation führen. So führt bei dem Rössleroszillator

$$
\begin{aligned}
& \dot{z}_{1}=2+z_{1}\left(z_{2}-4\right) \\
& \dot{z}_{2}=-z_{1}-z_{3} \\
& \dot{z}_{3}=z_{2}+0.45 z_{3}
\end{aligned}
$$

nur die Zerlegung $w=\left(z_{1}, z_{2}\right)^{t r}, v=z_{3}$ zur identischen Synchronisation.

\subsubsection{Einfügen von Dissipation}

Eine der wichtigsten Kopplungsarten von dynamischen Systemen, die zur Synchronisation von unidirektional und bidirektional gekoppelten (chaotischen) Oszillatoren, Felder gekoppelter Oszillatoren, ringförmige Strukturen, etc. oft eingesetzt wird, ist die „dissipative" Kopplung1. Die Methode wurde in der chaotischen Synchronisation als erste von Fujisaka \& Yamada [32] benutzt, Pyragas [107] hat sie dann zur Stabilisierung von periodischen Orbits im Bereich der Kontrolle von Chaos erfolgreich angewendet.

Die Idee besteht darin, in das Vektorfeld aus Glg. (3.5) Dissipation durch einen Rückkopplungsmechanismus einzufügen, der beim Erreichen der Zieldynamik, z.B. identische

${ }^{1}$ Diese Kopplungsvariante wird in der Literatur oft auch als Differenzkopplung oder diffusive Kopplung bezeichnet. 
Synchronisation, verschwindet. Der Fall von unidirektional gekoppelten Systemen läßt sich durch

$$
\begin{aligned}
\dot{\mathbf{x}} & =\mathbf{f}(\mathbf{x}) \\
\dot{\mathbf{x}} & =\mathbf{x}(\mathbf{y})+\underline{\underline{\mathbf{A}}}(\mathbf{h}(\mathbf{x})-\mathbf{h}(\mathbf{y}))
\end{aligned}
$$

beschreiben, mit $\underline{\underline{\mathbf{A}}}(\mathbf{h}(\mathbf{x})-\mathbf{h}(\mathbf{y}))$ als dissipativen Term, wobei die Matrix $\underline{\underline{\mathbf{A}}}$ die Kopplungsgewichte der einzelnen Rückkopplungsterme bestimmt. Die Rückkopplung des vollen Zustandsvektors („full state feedback“) wird oft in der Kontrolltheorie [107] benutzt. Bei der chaotischen Synchronisation möchte man mit möglichst wenigen Kopplungssignalen $s$ auskommen. Im häufig auftretenden Spezialfall von einem skalaren Kopplungsignal $h(\mathbf{x})$, welches darüberhinaus in nur einer Komponente von $\mathbf{f}(\mathbf{y})$ wirksam ist, besitzt $\underline{\underline{\mathbf{A}}}$ nur ein Element $a_{i j} \neq 0$. Zur identischen Synchronisation ist es aber oft ausreichend den Rückkopplungsterm in nur einer Komponente von $\mathbf{f}$ einzubringen. So läßt sich das Rösslersystem aus Glg. (3.4) mit Rückkopplung von $s=z_{3}$ in der $x_{3}$-Komponente durch

$$
\begin{aligned}
& \dot{x}_{1}=2+x_{1}\left(x_{2}-4\right) \\
& \dot{x}_{2}=-x_{1}-x_{3} \\
& \dot{x}_{3}=x_{2}+0.45 x_{3}+a_{3}\left(x_{3}-s\right)
\end{aligned}
$$

synchronisieren. Für $a_{3}=1.5$ wurde numerisch und auf einem Analogrechner robuste Synchronisation beobachtet [53].

Ein Vorteil der dissipativen Kopplung ist, daß nach dem Erreichen des synchronen Zustandes $\mathbf{x} \equiv \mathbf{z}$ die Steuerkraft, im Gegensatz zur Kopplung durch stabile Subsysteme in Abschn. 3.1.1, verschwindet, so daß nur eingegriffen wird falls sich das getriebene System von der Zieldynamik entfernt. Im Experiment werden die Systeme, verursacht z.B. durch Rauschen, Parameterunterschiede, etc., nie perfekt synchronisieren können, aber der synchrone Zustand wird dann durch beliebig kleine Kontrollkräfte $\sim\left(x_{i}-x_{i}\right) \approx 0$ stabilisiert. Diese Eigenschaft wird auch in der nichtlinearen Kontrolltheorie zur Stabilisierung von instabilen periodischen Orbits mit verschwindend kleinen Kontrollkräften benutzt.

Chaotische Synchronisation (IS oder GS) kann nicht für beliebig kleine Kopplungsstärken $a_{i} \approx 0$ auftreten, da der synchrone Zustand transversal instabil ist. Andererseits können zu starke Eingriffe auf das System auch destabilisierend wirken, so daß typischerweise eine optimale Kopplungsstärke existiert, die bei gewähltem Kopplungsterm zur robustesten Synchronisation, im Sinne von größter Stabilität von $\mathcal{M}_{\text {synch }}$, führt.

\subsubsection{Aktiv-Passiv Zerlegung}

Ein allgemeiner Ansatz zur Konstruktion von synchronisierten unidirektionalen gekoppelten dynamischen Systemen, der auf einer Zerlegung des Vektorfeldes $\mathbf{f}$ in einen aktiven und passiven Anteil beruht, wurde in [93, 97] eingeführt. Es wird von einem autonomen (chaotischen) dynamischen System

$$
\dot{\mathbf{z}}=\mathbf{F}(\mathbf{z})
$$




\section{Die treibende Kraft}

aus gestartet. Die Hauptidee liegt darin, dieses System rein formal in ein nichtautonomes System umzuschreiben

$$
\dot{\mathbf{x}}=\mathbf{f}(\mathbf{x}, \mathbf{s}(t)),
$$

mit $\mathbf{x}$ als neuem Zustandsvektor und $\mathbf{s}(t)$ als einer vorerst beliebigen vektorwertigen Funktion, die durch

$$
\mathbf{s}(t)=\mathbf{h}(\mathbf{x}) \quad \text { oder } \quad \dot{\mathbf{s}}(t)=\mathbf{h}(\mathbf{x}, \mathbf{s})
$$

definiert wird. Einsetzen von $\mathbf{s}$ in Glg. (3.9) liefert wieder das ursprüngliche System aus Glg. (3.8), $\mathbf{F}(\mathbf{x})=\mathbf{f}(\mathbf{x}, \mathbf{h}(\mathbf{x}))$, so daß jetzt zwei äquivalente Systeme mit derselben Dynamik vorliegen.

$$
\dot{\mathbf{z}}=\mathbf{F}(\mathbf{z}) \longleftrightarrow\left\{\begin{aligned}
\dot{\mathbf{x}} & =\mathbf{f}(\mathbf{x}, \mathbf{s}(t)) \\
\mathbf{s}(t) & =\mathbf{h}(\mathbf{x}) \quad \text { bzw. } \quad \dot{\mathbf{s}}(t)=\mathbf{h}(\mathbf{x}, \mathbf{s})
\end{aligned}\right.
$$

Sei nun

$$
\dot{\mathbf{y}}=\mathbf{f}(\mathbf{y}, \mathbf{s}(t))
$$

eine identische Kopie vom nichtautonomen Vektorfeld aus (3.9), so liegt die Aufgabe nun darin eine Funktion $\mathbf{h}(\mathbf{x})$ zu finden, so daß die beiden Systeme (3.9) und (3.11) bei gleichem treibenden Signal s aus (3.10) aber verschiedenen Anfangszuständen miteinander synchronisieren. Die Zerlegung von $\mathbf{F}$ in $\mathbf{f}, \mathbf{h}$ führt zur Synchronisation, falls die transversale Dynamik

$$
\dot{\mathbf{x}_{\perp}}=\mathbf{f}\left(\mathbf{x}+\mathbf{x}_{\perp}, \mathbf{s}(t)\right)-\mathbf{f}(\mathbf{x}, \mathbf{s}(t))
$$

einen asymptotisch stabilen Fixpunkt im Ursprung besitzt. Dies kann, wie in Abschnitt 2.1 diskutiert, durch Konstruktion einer Lyapunovfunktion, lineare Stabilitätsanalyse oder durch Berechnung von bedingten Lyapunovexponenten nachgewiesen werden.

Das versklavte System $\dot{\mathbf{x}}=\mathbf{f}(\mathbf{x}, \mathbf{s}(t))$ besitzt keine eigenen angeregten Freiheitsgrade mehr und wird deshalb als passiv bezeichnet. Zusammen mit dem treibenden (aktiven) System wird eine Zerlegung von $\mathbf{F}$ definiert, die Aktiv-Passiv-Zerlegung oder APD (,active-passive-decomposition“) von F genannt wird. Das Prinzip ist nochmals in Abb. 3.1 veranschaulicht. Durch die einseitige Kopplung entstehen scheinbar zwei verschiedene (das sich frei entwickelnde und das versklavte) Systeme, die durch die APD als zwei identische Systeme, die von dem gleichen Signal getrieben werden, aufgefaßt werden können. Die transversale Stabilität der Synchronisationsmannigfaltigkeit ist ausschliesslich durch das passive System (3.9) bestimmt, welches per Definition einen asymptotisch stabilen Fixpunkt in $\mathbf{0}$ besitzt, womit die Synchronisation von (3.9) mit (3.11) garantiert ist. Als Beispiel betrachten wir eine APD zweier unidirektional gekoppelter Rössleroszillatoren 


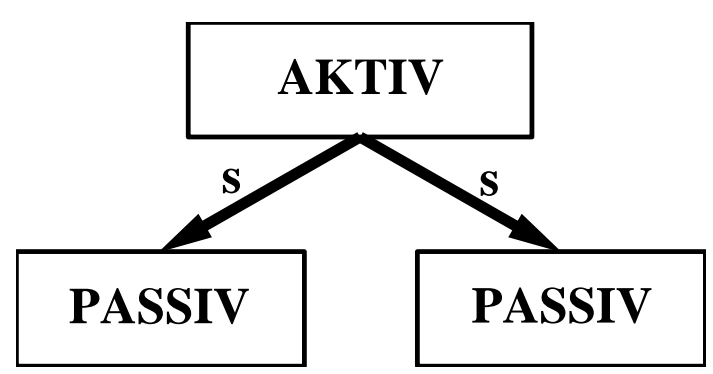

Abbildung 3.1.: Aktiv-Passiv-Zerlegung von gekoppelten Systemen.

$$
\begin{aligned}
& \dot{x}_{1}=2+-4 x_{1}+x_{2}^{2}-s x_{2} \\
& \dot{x}_{2}=-x_{2}-x_{3}+s \\
& \dot{x}_{3}=x_{2}+0.45 x_{3}
\end{aligned}
$$$$
\dot{y}_{1}=2+-4 y_{1}+y_{2}^{2}-s y_{2}
$$$$
\dot{y}_{2}=-y_{2}-y_{3}+s
$$$$
\dot{y}_{3}=y_{2}+0.45 y_{3} \text {, }
$$

mit

$$
s=x_{2}-x_{1}
$$

als aktiven Part und Übertragungssignal ${ }^{2}$. Den Nachweis der Passivität von Glg. (3.13) führen wir über eine lineare Stabilitätsuntersuchung des transversalen Systems $\mathbf{e}:=\mathbf{x}_{\perp}$

$$
\begin{aligned}
& \dot{e}_{1}=-4 e_{1}+e_{2}\left(x_{2}+y_{2}-s\right) \\
& \dot{e}_{2}=-e_{2}-e_{3} \\
& \dot{e}_{3}=e_{2}+0.45 e_{3} .
\end{aligned}
$$

Die $e_{1}$-Koordinate von Glg. (3.15) entkoppelt von den restlichen beiden, so daß man zuerst die Stabilität des $\left(e_{2}-e_{3}\right)$-Subsystems alleine untersuchen kann. Die lineare Stabilitätsuntersuchung liefert die Eigenwerte $\lambda_{2,3}=-0.275 \pm i \sqrt{0.474}$, womit das Subsystem asymptotisch stabil ist und $e_{2}$ und $e_{3}$ für $t \rightarrow \infty$ gegen 0 konvergieren. Die Gleichung für $e_{1}$ reduziert sich dann auf $\dot{e}_{1}=-4 e_{1}$. Damit verschwindet auch $e_{1}$ und das System Glg. (3.13) hat einen asymptotisch stabilen Fixpunkt im Ursprung und ist demnach passiv. Anzumerken ist, daß der Nachweis unabhängig vom treibenden Signal geführt werden konnte, so daß beide Systeme in (3.13) nicht nur mit dem Signal aus Glg. (3.14), sondern getrieben von beliebigen Signalen miteinander synchronisieren!

Aufgrund der allgemeinen Formulierung der APD kann gezeigt werden [93], daß die meisten existieren Kopplungschemata für unidirektionale Systeme auch eine APD definieren. In [93] wurde die Äquivalenz, von der Kopplung mit Subsystemen (Abschn. 3.1.1), der disispativen Kopplung (Abschn. 3.1.2) und weiterer Varianten, zur APD nachgewiesen.

\footnotetext{
${ }^{2}$ Einsetzen von Glg. (3.14) in Glg. (3.13) führt auf die Originalrösslergleichungen. Diese Darstellung unterstreicht nochmals, daß durch die APD das treibende System nur formal umgeschrieben wird, während das getriebene eine reale Kopplungskraft erfährt.
} 


\section{Die treibende Kraft}

\subsubsection{Diskret in der Zeit}

Die bisher vorgestellten Kopplungsmechanismen haben alle kontinuierliche Kopplungssignale benutzt. Im Hinblick auf praktische Anwendungen, wie z.B. die momentan diskutierte (sichere) Nachrichtenübertragung unter Benutzung chaotischer Synchronisation [39, 87, 94, 97], ist es schwierig kontinuierliche Signale über bandbreitenbegrenzte Kanäle zu übertragen, mit denen dann ein anderes chaotisches System synchronisieren soll. Mit dem Verfahren aus [96, 124, 125] ist es möglich kontinuierliche Systeme, beschrieben durch gewöhnliche Differentialgleichungen, mit einer zeitdiskreten Folge von Kopplungswerten perfekt zu synchronisieren. Die allgemeinste Formulierung von der diskreten Kopplung, auch „sporadic driving“ genannt, beruht auf einer gegebenen AktivPassiv-Zerlegung eines kontinuierlichen Systems

$$
\begin{aligned}
\dot{\mathbf{x}} & =\mathbf{f}(\mathbf{x}, \mathbf{s}(t)) \\
\mathbf{s}(t) & =\mathbf{h}(\mathbf{x}) .
\end{aligned}
$$

Dies System treibt nun eine identische Kopie

$$
\begin{aligned}
& \dot{\mathbf{y}}=\mathbf{f}(\mathbf{y}, \mathbf{u}) \\
& \mathbf{u}=\left\{\begin{array}{lll}
\mathbf{s}(t) & \text { : für } t=n T \\
\mathbf{h}(\mathbf{y}) & \text { : für } t \neq n T,
\end{array}\right.
\end{aligned}
$$

wobei das aktive System $\mathbf{h}(\mathbf{x})$ jetzt nur zu Vielfachen der vorgegebenen „Abtastzeit" $T \geq 0$ Einfluß auf das getriebene System hat, so daß es sich zu allen anderen Zeiten frei entwickeln kann. Für $T \rightarrow 0$ geht das sporadic driving in eine normale APD von kontinuierlichen Systemen über. Aufgrund der nur zu diskreten Zeiten vorhandenen Kopplung, sind zur Stabilitätsuntersuchung von $\mathbf{x}_{\perp}$ für beliebige Abtastzeiten die klassischen Methoden wie Nachweis der asymptotischen Stabilität eines Fixpunktes oder Konstruktion einer Lyapunovfunktion aus Abschn. 2.1 in der Regel nicht mehr anwendbar, und man kann die Stabilität von $\mathbf{x}_{\perp}$ meist nur durch Berechnung des größten bedingten Lyapunovexponenten nachweisen. Für $T \rightarrow 0$ wurde in [96] gezeigt, daß sich das Stabilitätsverhalten des zeitkontinuierlich getriebenen Systems auf das zeitdiskret getriebene System übertragen läßt. Das bedeutet, daß alle APDs mit kontinierlichem Kopplungssignal auch bei hinreichend feiner Abtastung mit einer zeitdiskreten Folge von Signalen auf Synchronisation führen. Das Konzept kann aber oft auch bei grob abgetasteten Zeitreihen erfolgreich angewendet werden.

Eine andere Variante ist, direkt eine oder mehrere Zustandsvariablen $y_{i}$ nach fest gewählten Zeitabständen $T$ durch den Wert im treibenden System $z_{i}$ vollständig oder partiell zu ersetzen, d.h.

$$
y_{i}(t)=\left\{\begin{array}{lll}
y_{i}+\varepsilon\left(z_{i}-y_{i}\right) & \varepsilon \in[0,1]: & \text { für } t=n T \\
y_{i}: & \text { für } t \neq n T
\end{array} .\right.
$$

Bei $\varepsilon=0$ sind die Systeme ungekoppelt und für $\varepsilon=1$ wird die Zustandsvariable periodisch auf den „richtigen“ Wert aufgefrischt. Man kann dies als eine zeitdiskrete Variante 
der Kopplung durch Subsysteme aus Abschn. 3.1.1 ansehen. Aber dieser Ansatz ist effektiver als man erwartet, so läßt sich das Rösslersystem aus Glg. (3.4), durch Ersetzen der $y_{2}$ Komponente mit $s=z_{2}$ alle $T=1.5$ Zeiteinheiten, synchronisieren. Bei kontinuierlichem Kopplungssignal $T=0$ führt die entsprechende Kopplung durch Subsysteme aber auf keine stabile Synchronisation.

\subsubsection{Der nichtlineare Beobachter}

Bei der Synchronisation versucht man typischerweise für ein gegebenes dynamisches System eine Meßgröße und zugehörige Kopplungsfunktion zu finden, die zu einer asymptotisch stabilen transversalen Dynamik führen. In der Kontroll- und Systemtheorie werden das dynamische System und die Observablen als fest vorgegeben vorrausgesetzt und man betrachtet das zusammengefaßte Gesamtsystem

$$
\begin{aligned}
\dot{\mathbf{x}}(t) & =\mathbf{f}(\mathbf{x}(t)), \quad \mathbf{x}(0)=\mathbf{x}_{0} \in \mathbb{R}^{n}, \quad t \geq 0 \\
\mathbf{s}(t) & =\mathbf{h}(\mathbf{x}(t)), \quad \mathbf{s} \in \mathbb{R}^{p}
\end{aligned}
$$

als Einheit. Dies ist dadurch motiviert, daß bei (technischen) Systemen oft nur Teile des Gesamtzustandes überhaupt einer Messung zugänglich sind. Für eine effektive Stabilisierung ist es aber oft erforderlich, daß möglichst der vollständige Zustand des Systems (3.19) bekannt ist. Ein zweites System

$$
\begin{aligned}
\dot{\tilde{\mathbf{x}}}(t) & =\tilde{\mathbf{f}}(\tilde{\mathbf{x}}(t), \mathbf{s}(t)), \quad \tilde{\mathbf{x}}(0)=\tilde{\mathbf{x}}_{0} \in \mathbb{R}^{n}, \quad t \geq 0 \\
\tilde{\mathbf{s}}(t) & =\mathbf{h}(\tilde{\mathbf{x}}(t)), \quad \tilde{\mathbf{s}} \in \mathbb{R}^{p},
\end{aligned}
$$

das den vollständigen Zustandes $\mathbf{x}$ aus den gemessenen Größen s rekonstruiert, wird in der Systemtheorie ein Beobachter („observer“) [69, 132] genannt. Zur Konstruktion eines Beobachters wird zuerst überprüft, ob in den Observablen $\mathbf{s}$ der vollständige Zustand $\mathbf{x}$ enthalten ist und damit prinzipiell auch rekonstruiert werden kann. Danach wird ein zweites System (3.20) konstruiert, so daß für $t \rightarrow \infty \tilde{\mathbf{x}}$ gegen $\mathbf{x}$ konvergiert. Offensichtlich ist die chaotische Synchronisation dynamischer Systeme eng verwandt mit dieser Teildisziplin der mathematischen Kontrolltheorie [76, 78, 105, 132]. Für lineare dynamische Systeme ist dieses Problems vollständig gelöst [69] worden, d.h. es existieren Sätze ob der Gesamtzustand $\mathbf{x}$ aus den Meßgrößen $\mathbf{s}$ beobachtbar ist und falls dies der Fall ist, wie man einen Beobachter konstruieren kann. Für nichtlineare dynamische Systeme kann bis jetzt kein geschlossener Lösungsweg angegeben werden. Bisherige Arbeiten zum nichtlinearen Beobachter können Resultate für nur wenige Systemklassen vorweisen [78, 105] und von einer allgemeinen Lösung ist man gegenwärtig noch weit entfernt.

Andererseits sollte fast jede skalare Meßgröße $s(t)$ von einem chaotischen System (3.19) ausreichend sein, um den vollständigen Systemzustand rekonstruieren zu können, dies folgt schon aus Takens Einbettungstheorem [117, 128]. Im Kontext der Synchronisation wird dies in [126] diskutiert. Das Einbettungstheorem liefert aber nur eine Existenzaussage des nichtlinearen Beobachters. Es sagt nichts darüber aus wie man die Einbettung in den Orignalzustandsraum von (3.19) zurücktransformieren kann, bzw. wie man einen 
solchen Beobachter bauen kann. Für iterierte Abbildungen wurde in [45] die Konstruktion eines nichtlinearen Beobachters vorgeschlagen, welcher außer dem aktuellen skalaren Meßsignal $s(n)=h\left(\mathbf{x}^{n}\right)$ auch noch die letzten $N$-Iterationen $s^{n-N+1}, \ldots, s^{n-1}$ zur Kopplung ausnutzt, wobei $N$ die Dimension der Abbildung $\mathbf{f}$ ist, wodurch implizit eine Einbettung zur Konstruktion des Beobachters verwendet wird. Es kann damit gezeigt werden, daß sich zumindest lokal mit dieser Strategie der Zustand vollständig rekonstruieren läßt. Zur Konstruktion eines globalen Beobachters müssen aber wieder einige restriktive Bedingungen an das System und die Meßfunktion $h(\mathbf{x})$ gestellt werden. Diese Arbeit stellt damit einen hoffnungsvollen Schritt zu einer systematischen Konstruktion von einem Beobachter, bzw. synchronisierendem System, von zeitdiskreten dynamischen Systemen dar. Auf zeitkontinuierliche System läßt sich die Methode aus [45] prinzipiell nicht anwenden, so daß das Problem der Konstruktion eines nichtlinearen Beobachters für diese Systemklasse weiterhin ungelöst ist, woran aber intensiv geforscht wird (siehe z.B. [63]).

\subsection{Direkte Kompensation des Schmetterlingseffektes}

Im vorangegangenem Abschnitt wurden die wichtigsten Kopplungsarten zur Beeinflussung der Systemdynamik von einem getriebenen dynamischen System vorgestellt und diskutiert. Allen gemein ist, daß das getriebene System durch eine im Phasenraum feste globale Kopplungsfunktion $\mathbf{C}(\mathbf{h}(\mathbf{x}), \mathbf{h}(\mathbf{y}))$ der Observablen $\mathbf{h}(\mathbf{x})$ vom treibenden und $\mathbf{h}(\mathbf{y})$ vom getriebenen System beeinflußt wird. Diese Kopplungsfunktion wird mehr oder weniger willkürlich gewählt, um die gewünschte Zieldynamik (IS, GS, PS, FS, UPO, .. ) zu erreichen. Es existieren aber wenig Arbeiten, die abhängig vom zu steuernden System, systematisch eine Kopplungsfunktion liefern, so daß die Wahl der Kopplung meist durch Ausprobieren geschieht.

Für die IS wurde ein mögliches Lösungsverfahren von Pecora et al. [51] vorgeschlagen. Als Kopplungsfunktion wird $\underline{\underline{\mathbf{A}}}(\mathbf{x}-\mathbf{y})$ angesetzt, wobei $\underline{\underline{\mathbf{A}}}$ eine $(n \times n)$-Matrix mit zu bestimmenden Kopplungsgewichten ist. Die Gewichte für die linearen Rückkopplungsterme werden durch globale Minimierung nach dem betragsmäßig größten Eigenwert von $\underline{\underline{J}} \dot{\mathbf{x}}_{\perp}=\underline{\underline{J}} \mathbf{f}(\mathbf{x})+\underline{\underline{\mathbf{A}}}$ bestimmt. Damit erhält man zwar eine sehr robuste (insbesondere gegen additives Rauschen) und für diesen Ansatz optimale Kopplung, welche aber erstens sehr rechenintensiv zu bestimmen ist ${ }^{3}$ und zweitens große Kopplungskräfte nach sich zieht. Weiterhin ist diese Methode nicht sehr elegant, da die Kopplungsfunktion bzw. Kopplungsmatrix hier eigentlich durch systematisches Ausprobieren bestimmt wird. Die in Abschnitt 3.1.5 vorgestellte Methode des nichtlinearen Beobachters liefert Aussagen ob mit der gemessenen Observable $\mathbf{s}=\mathbf{h}(\mathbf{x})$ Synchronisation prinzipiell möglich ist und benutzt dann Methoden der nichtlinearen Kontrolltheorie um eine erfolgreiche Kopplungsfunktion zu bestimmen.

Wünschenswert wäre ein Verfahren, welches bei gegebenen dynamischen System syste-

${ }^{3}$ Es müssen die $n^{2}$ Elemente der Kopplungsmatrix $\underline{\underline{\mathbf{A}}}$ bestimmt werden, was auf ein oft hochgradig schlecht konditioniertes Minimierungsproblem führt, so daß man meist auf aufwendige numerische Verfahren wie „simulated annealing“ oder evolutionäre Algorithmen zurückgreifen muß. 
matisch

1. eine Observable $\mathbf{s}=\mathbf{h}(\mathbf{x})$ mit $1 \leq \operatorname{dim}\{\mathbf{s}\} \ll \operatorname{dim}\{\mathbf{x}\}$

2. und eine geeignete Kopplungsfunktion

liefert, mit denen identische Synchronisation erreicht werden kann. Im Verlaufe des Restes dieses Kapitels wird eine Kopplungsmethode vorgestellt, die diesen Anforderungen genügt und zusätzlich noch sehr gute Stabilitätseigenschaften besitzt.

\subsubsection{Dynamische Kopplung im Phasenraum}

Eine wesentliche Einschränkung der in Abschn. 3.1 vorgestellten Kopplungsverfahren ist, daß eine feste (statische) global gültige Funktion der Observablen im Phasenraum benutzt wird. Dies wird deutlich, wenn man sich erinnert, daß die sensitive Abhängigkeit von den Anfangsbedingungen auf dem Attraktor in Betrag und Richtung variiert. So existieren auf den meisten Attraktoren Gebiete, die nur schwach instabil sind, während andere (bevorzugt in der Nähe von instabilen Orbits niedriger Periode [46]) zu starker Expansion des Abstandes benachbarter Trajektorien führen. Weiterhin sind die lokalen Expansionsrichtungen entlang des chaotischen Flusses nicht konstant, sondern verändern sich ständig während der Zeitentwicklung eines Zustandes. Dies kann dazu führen, daß eine Kopplungsfunktion an einigen Gebieten auf dem Attraktor sehr effektiv steuert, während sie in anderen ohne Wirkung bleibt, da sich die lokalen Stabilitätseigenschaften geändert haben. Wenn man nun die Forderung nach einer im gesamten Phasenraum festen Kopplungsfunktion fallenläßt, kann man lokal variable bzw. dynamische Kopplungsfunktionen einführen $\mathbf{C}_{\mathbf{y}}\left(\mathbf{h}_{\mathbf{x}}(\mathbf{x}), \mathbf{h}_{\mathbf{y}}(\mathbf{y}), \mathbf{x}, \mathbf{y}\right)$, welche den lokalen Stabilitätseigenschaften auf dem Attraktor angepaßt werden können. Der wesentliche Unterschied zu der oben diskutierten festen Kopplungsfunktion ist, daß nun $\mathbf{C}_{\mathbf{y}}$ und die Meßfunktionen $\mathbf{h}_{\mathbf{x}}, \mathbf{h}_{\mathbf{y}}$ direkt vom aktuellen Systemzustand $\mathbf{x}, \mathbf{y}$ abhängen können, welches wir zur Unterscheidung durch die Indizierung mit dem Zustand andeuten ${ }^{4}$.

Die Systeme liegen nun allgemein als zwei gekoppelte ODEs der Form

$$
\begin{aligned}
\dot{\mathbf{x}} & =\mathbf{f}(\mathbf{x})+\mathbf{C}_{\mathbf{x}}\left(\mathbf{h}_{\mathbf{y}}(\mathbf{y}), \mathbf{h}_{\mathbf{x}}(\mathbf{x})\right) \\
\dot{\mathbf{y}} & =\mathbf{f}(\mathbf{y})+\mathbf{C}_{\mathbf{y}}\left(\mathbf{h}_{\mathbf{x}}(\mathbf{x}), \mathbf{h}_{\mathbf{y}}(\mathbf{y})\right)
\end{aligned}
$$

bzw. zwei gekoppelte iterierte Abbildungen der Form

$$
\begin{aligned}
& \mathbf{x}^{n+1}=\mathbf{f}\left(\mathbf{x}^{n}\right)+\mathbf{C}_{\mathbf{x}}\left(\mathbf{h}_{\mathbf{y}}\left(\mathbf{y}^{n}\right), \mathbf{h}_{\mathbf{x}}\left(\mathbf{x}^{n}\right)\right) \\
& \mathbf{y}^{n+1}=\mathbf{f}\left(\mathbf{y}^{n}\right)+\mathbf{C}_{\mathbf{y}}\left(\mathbf{h}_{\mathbf{x}}\left(\mathbf{x}^{n}\right), \mathbf{h}_{\mathbf{y}}\left(\mathbf{y}^{n}\right)\right)
\end{aligned}
$$

vor. Lorenz hatte den sogenannten Schmetterlingseffekt als Metapher zur Verdeutlichung der sensitiven Abhängigkeit von den Anfangsbedingungen von chaotischen Systemen

\footnotetext{
${ }^{4}$ Formal gesehen, läßt sich auch die dynamische Kopplung in der Form $\mathbf{C}(\mathbf{h}(\mathbf{x}), \mathbf{h}(\mathbf{y}))$ mathematisch korrekt darstellen, da ihre funktionale Form nur vom Zustand $\mathbf{x}$ bestimmt wird. Im Hinblick auf reale Anwendbarkeit, ist es aber üblich und sinnvoll zwischen Meßgröße $\mathbf{h}_{\mathbf{x}}$ und Steuergröße $\mathbf{C}_{\mathbf{x}} \mathbf{z u}$ unterscheiden.
} 


\section{Die treibende Kraft}

eingeführt. Lokale (infinitesimal) kleine Abweichungen im Anfangszustand zweier identischer Systeme führen unweigerlich im Laufe der Zeitentwicklung zur exponentiellen Divergenz der Trajektorien und damit zur Unmöglichkeit von Langzeitvorhersagen der Zustände. Bei der chaotischen Synchronisation wird dieser Divergenz durch Einführung einer geeigneten Kopplung zwischen den Systemen entgegengewirkt. Mit den klassischen statischen Kopplungsfunktionen wird eine dissipative Kraft eingeführt welche nur im Mittel das Auseinanderlaufen der Trajektorien verhindern kann, d.h. es können weiterhin Untermengen auf dem Attraktor existieren welche lokal instabil sind (siehe Abschn. 2.1.1). Die dynamische Kopplungsmethode in Glg. (3.21) und Glg. (3.22) hat das Potential direkt der lokalen Instabilität entgegenzuwirken und ist bestrebt das getriebene System in jedem Punkt auf dem Attraktor stabil zu machen, womit die sensitive Abhängigkeit von den Anfangsbedingungen global unterdrückt werden kann ${ }^{5}$.

Im Folgenden wollen wir eine geeignete lokale Kopplungsfunktion $\mathbf{C}_{\mathbf{y}}$ konstruieren. Das dynamische zeitdiskrete System in Glg. (3.22) liefert direkt den Fluß $\Phi^{t}$, während im zeitkontinuierlichen Fall in Glg. (3.21) im Phasenraum $\mathbb{R}^{n}$ ein Vektorfeld f, welches das Geschwindigkeitsfeld des Phasenflusses $\Phi^{t}$ darstellt, definiert wird. Die Divergenz $\operatorname{divf}(\mathbf{x})=\operatorname{Sp}\{\underline{\underline{J}}(\mathbf{x})\}=\operatorname{Sp}\left\{\frac{\partial \mathbf{f}}{\partial \mathbf{x}}\right\}$ bestimmt damit die Geschwindigkeit mit der sich die Größe eines infinitesimalen Volumenelementes an der Stelle $\mathbf{x}$ unter der Wirkung von $\Phi^{t}$ ändert. Bei den hier betrachteten dissipativen Systemen ist diese Größe im Mittel kleiner Null, so daß das Volumenenelement im Mittel kontrahiert wird. Betrachtet man nun aber die Zeitentwicklung dieses Elementes so zeigt sich, daß es in einigen Richtungen $\mathbf{u}^{i}(\mathbf{x})$ gestreckt wird ${ }^{6}$. In den verbleibenden Richtungen $\mathbf{u}^{s}(\mathbf{x})$ wird diese Streckung überkompensiert, so daß das Volumen insgesamt kontrahiert wird. Der Einfachheit halber behandeln wir zuerst den Fall nur einer streckenden Richtung $\mathbf{u}^{i}(\mathbf{x})$, welcher z.B. bei 3-dimensionalen chaotischen ODEs wie dem Lorenz- oder Rösslersystem auftritt. Betrachtet man nun den Abstand zweier benachbarte Zustände $\|\mathbf{e}\|=\|\mathbf{x}-\mathbf{y}\|<\delta$, so wird nur der Anteil des Abstandes in der instabilen Richtung $\left\langle\mathbf{u}^{i} \mid \mathbf{e}\right\rangle=\left\langle\mathbf{u}^{i} \mid(\mathbf{x}-\mathbf{y})\right\rangle$ um einen noch zu bestimmenden Faktor $\gamma_{\mathbf{x}}>1$, der auch vom aktuellen Zustand abhängen kann, gestreckt. Zur Synchronisation muß genau diese Streckung kompensiert werden. Der restliche Anteil liegt im stabilen Unterraum und wird aufgrund der Dissipation vom System selbst kontrahiert. Somit machen wir für die lokale Kopplungsfunktion folgenden ersten Ansatz

$$
\begin{aligned}
& \mathbf{C}_{\mathbf{x}}\left(\mathbf{h}_{\mathbf{y}}(\mathbf{y}), \mathbf{h}_{\mathbf{x}}(\mathbf{x})\right)=\gamma_{\mathbf{x}}\left\langle\mathbf{u}_{\mathbf{x}}^{i} \mid(\mathbf{y}-\mathbf{x})\right\rangle \mathbf{u}_{\mathbf{x}}^{i} \\
& \mathbf{C}_{\mathbf{y}}\left(\mathbf{h}_{\mathbf{x}}(\mathbf{x}), \mathbf{h}_{\mathbf{y}}(\mathbf{y})\right)=\gamma_{\mathbf{y}}\left\langle\mathbf{u}_{\mathbf{y}}^{i} \mid(\mathbf{x}-\mathbf{y})\right\rangle \mathbf{u}_{\mathbf{y}}^{i} .
\end{aligned}
$$

In transversalen Koordinaten $\mathbf{x}_{\perp}=\frac{1}{2}(\mathbf{x}-\mathbf{y})$ schreibt sich der Kopplungsterm aus Glg. (3.23) $\mathbf{C} \sim\left\langle\mathbf{u}^{i} \mid \mathbf{x}_{\perp}\right\rangle \mathbf{u}^{i}$. In dieser Schreibweise wird deutlich, daß die Kopplung exakt

\footnotetext{
${ }^{5}$ Jedes einzelne System für sich betrachtet bleibt aber natürlich weiterhin nur für kurze Zeit vorhersagbar. Die chaotische Synchronisation bewirkt keinen Vorteil in der Vorhersage der Zeitentwicklung des Systems, sondern es wird der vollständige Zustand des treibenden Systems zum jetzigen Zeitpunkt vorhergesagt, wobei in Form der Meßsignale nur partielle Information über den Zustand des Treibers vorliegt.

${ }^{6}$ Wie oben diskutiert bleiben diese Richtungen nicht konstant, sondern verändern sich stetig entlang des Flusses $\Phi^{t}$.
} 
das tut was sie soll.

- Sie wirkt nur auf den Anteil von $\mathbf{x}_{\perp}$ der abstoßend ist. Das heißt, die Kopplung nutzt implizit die lokalen Kontraktionseigenschaften des getriebenen Systems aus und wirkt nur auf die Anteile von $\mathbf{x}_{\perp}$ die entlang des Flusses $\Phi^{t}$ expandiert werden. Den verbleibenden Anteil kontrahiert das getriebene System selber!

Von Standpunkt der Theorie der dynamischen Systeme kann man damit diesen Ansatz als optimal ansehen, da die Kopplung durch die Systemdynamik definiert wird und auch als minimal, da sie darüberhinaus diese für ihre Zwecke weitestmöglich ausnutzt. Aus diesem Grund bezeichnen wir im Folgenden diese Kopplungstrategie als (lokal) dynamische Kopplung.

Ein Problem ist nun noch, daß der Kopplungsterm Glg. (3.23) die vollständigen $n$-dimensionalen Zustände zur Kopplung benötigt, welche durch die Synchronisation ja eigentlich erst vorhergesagt werden sollte. Eine gute Kopplung sollte mittels minimaler Information in Form von möglichst wenigen skalaren Übertragungssignalen den kompletten Zustand des treibenden Systems rekonstruieren, denn wenn dieser als Signal schon komplett vorliegt, ist es Unsinn denselben nochmals durch eine komplizierte Strategie zu reproduzieren. Falls das Vektorfeld $\mathbf{f}$ zumindest stetig differenzierbar $\mathbf{f} \in \mathrm{C}^{1}$ ist, dann ist der lokale Fluß $\Phi^{t}$ strukurell stabil gegenüber kleinen Störungen in den Parametern und dem Zustand. Das bedeutet, daß benachbarte Zustände im Phasenraum $\|\mathbf{e}\|=\|\mathbf{x}-\mathbf{y}\|<\delta$ auch ähnliche lokale Stabilitätseigenschaften besitzen und $\mathbf{u}_{\mathbf{x}}^{i} \approx \mathbf{u}_{\mathbf{y}}^{i}$ angenommen werden darf. Der Anteil von $\mathbf{e}$ an $\mathbf{u}_{\mathbf{y}}^{i}$ läßt sich dann durch $\left.\left\langle\mathbf{u}_{\mathbf{y}}^{i} \mid(\mathbf{x}-\mathbf{y})\right\rangle \mathbf{u}_{\mathbf{y}}^{i} \approx\left(\left\langle\mathbf{u}_{\mathbf{x}}^{i} \mid \mathbf{x}\right\rangle-\left\langle\mathbf{u}_{\mathbf{y}}^{i}\right| \mathbf{y}\right)\right\rangle \mathbf{u}_{\mathbf{y}}^{i}$ approximieren. Für den Fall einer instabilen Richtung ergibt sich damit für zwei gekoppelte ODEs folgendes Synchronisationsschema

$$
\begin{aligned}
\dot{\mathbf{x}} & =\mathbf{f}(\mathbf{x})+\gamma_{\mathbf{x}}\left(s_{\mathbf{y}}-s_{\mathbf{x}}\right) \mathbf{u}_{\mathbf{x}}^{i} \\
\dot{\mathbf{y}} & =\mathbf{f}(\mathbf{y})+\gamma_{\mathbf{y}}\left(s_{\mathbf{x}}-s_{\mathbf{y}}\right) \mathbf{u}_{\mathbf{y}}^{i}
\end{aligned}
$$

mit

$$
s_{\mathbf{x}}=\mathbf{h}(\mathbf{x})=\left\langle\mathbf{u}_{\mathbf{x}}^{i} \mid \mathbf{x}\right\rangle \quad \text { und } \quad s_{\mathbf{y}}=\mathbf{h}(\mathbf{y})=\left\langle\mathbf{u}_{\mathbf{y}}^{i} \mid \mathbf{y}\right\rangle
$$

als skalare Kopplungssignale und

$$
\begin{aligned}
& \mathbf{C}_{\mathbf{x}}=\gamma_{\mathbf{x}}\left(s_{\mathbf{y}}-s_{\mathbf{x}}\right) \mathbf{u}_{\mathbf{x}}^{i} \\
& \mathbf{C}_{\mathbf{y}}=\gamma_{\mathbf{y}}\left(s_{\mathbf{x}}-s_{\mathbf{y}}\right) \mathbf{u}_{\mathbf{y}}^{i}
\end{aligned}
$$

als Kopplungsfunktionen für Glg. (3.24). Die Kopplungsstärke $\gamma_{\mathbf{x}}$ sollte so gewählt werden, daß sie mindestens die lokale Instabilität des aktuellen Zustandes $\mathbf{x}$ kompensieren kann. Das Synchronisationschema für iterierte Abbildungen wird analog gebildet. Was nun noch verbleibt, ist die instabilen Richtungen $\mathbf{u}^{i}$ und die Kopplungsstärke $\gamma$ für ein vorgegebenes ansonsten aber beliebiges System

$$
\begin{aligned}
& \dot{\mathbf{x}}=\mathbf{f}(\mathbf{x}) \quad \text { bzw. } \\
& \mathbf{x}^{n+1}=\mathbf{f}\left(\mathbf{x}^{n}\right)
\end{aligned}
$$

zu bestimmen. 


\subsubsection{Lokale Maße der Instabilität von Systemzuständen}

Für ein dynamisches System können die lokalen Stabilitätseigenschaften einer Trajektorie entlang des Flusses $\Phi^{t}(\mathbf{x})$ in einer $\varepsilon$-Umgebung $\mathcal{U}_{\varepsilon}(\mathbf{x})$ um $\mathbf{x}$, durch die der linearisierten Flußabbildung charakterisiert werden ${ }^{7}$. Damit kann alle benötigte Information über die lokal kontrahierenden und expandierenden Richtungen von $\mathbf{x}$ aus der Jacobimatrix der Flußabbildung $\underline{\underline{J}} \Phi^{t}(\mathbf{x})$ gewonnen werden. Bei iterierten Abbildungen ist der Fluß explizit durch die Abbildungsvorschrift $\Phi: \mathbf{x}^{n} \mapsto \mathbf{x}^{n+1}=\mathbf{f}\left(\mathbf{x}^{n}\right)$ gegeben, so daß in diesem Fall

$$
\underline{\underline{J}} \Phi\left(\mathbf{x}^{n}\right)=\left.\underline{\underline{J}} \mathbf{f}(\tilde{\mathbf{x}})\right|_{\tilde{\mathbf{x}}=\mathbf{x}^{n}}=\left.\frac{\partial \mathbf{f}}{\partial \tilde{\mathbf{x}}}\right|_{\tilde{\mathbf{x}}=\mathbf{x}^{n}}
$$

zur Bestimmung der expandierenden Richtungen verwendet werden kann. Im zeitkontinuierlichen Fall (ODEs) ist die Flußabbildung nicht explizit gegeben und kann i.A. nur numerisch approximiert werden. Die folgenden Ableitungen sind streng genommen nur für den Fall iterierter Abbildungen gültig, wo der Fluß explizit vorliegt und durch die Jacobimatrix $\underline{J}:=\underline{J} \Phi^{t}(\mathbf{x})$ approximiert werden kann. Das Kopplungsschema für ODEs kann aber in Abschn. 3.2.5 bis auf eine Konstante auf den Fall iterierter Abbildungen zurückgeführt werden, so daß die Ergebnisse der folgenden Ableitungen auch bei ODEs gültig bleiben.

\section{Lokale Lyapunovexponenten}

Wenn man Maße der Instabilität von Trajektorien sucht, wird man wohl als erstes an Lyapunovexponenten und Lyapunovektoren denken. Die Berechnung erfolgt durch simultanes Lösen der Differentialgleichung

$$
\dot{\mathbf{e}}=\left.\frac{\partial \mathbf{f}}{\partial \tilde{\mathbf{x}}}\right|_{\tilde{\mathbf{x}}=\mathbf{x}} \mathbf{e}
$$

bzw. der iterierten Abbildung

$$
\mathbf{e}^{n+1}=\left.\frac{\partial \mathbf{f}}{\partial \tilde{\mathbf{x}}}\right|_{\tilde{\mathbf{x}}^{n}=\mathbf{x}^{n}} \mathbf{e}^{n}
$$

zum Originalgleichungssystem (3.27) bzw. (3.28). Ein vorgegebener Tangentialvektor e stellt sich im Laufe der Zeit in Richtung der größten Expansion ein und der zugehörige Lyapunovexponent läßt sich aus der Norm von e bestimmen. Die aus Glg. (3.30) bzw. Glg. (3.31) gewonnenen Exponenten und Richtungen sind Mittelwerte über das gesamte invariante Maß. Man kann diese Größen aber auch lokal definieren, indem man Mittelwerte über kurze Zeiten berechnet. Da sich aber die Tangentialvektoren nicht instantan den Stabilitätseigenschaften des Flusses in einem Punkt $\mathbf{x}$ anpassen können, weisen die Tangentialvektoren i.A. nicht in die Richtungen der aktuellen Instabilität, sondern haben

\footnotetext{
${ }^{7}$ Dies ist sicher falls $\Phi^{t}(\mathbf{x})$ zumindest stetig differenzierbar ist. Die Vorraussetzungen können noch abgeschwächt werden und die Aussage bleibt gültig, falls die Linearisierung $\underline{\underline{J}} \Phi^{t}$ von $\Phi$ in $\mathcal{U}_{\varepsilon}(\mathbf{x})$ nichtsingulär ist und $\lim \sup _{\|\mathbf{x}\| \rightarrow 0, t \geq 0} \frac{\left\|\mathbf{f}(\mathbf{x})-\underline{J} \Phi^{t}(\mathbf{x})\right\|}{\|\mathbf{x}\|}=0$ gilt.
} 


\section{Die treibende Kraft}

noch Anteile der Richtungen der Instabilität aus der vergangenen Zeitentwicklung. Wir brauchen aber Information über die instantanen Stabilitätseigenschaften des Flusses, weswegen dieser Ansatz für unsere Zwecke nicht geeignet ist.

\section{Eigenvektoren und Eigenwerte}

Eine bessere Wahl ist Eigenwertzerlegung der Jacobimatrix aus Glg. (3.29)

$$
\begin{aligned}
\underline{\underline{J}} \underline{\underline{\mathbf{V}}} & =\underline{\underline{\mathbf{V}}} \underline{\underline{\Lambda}} \quad b z w . \\
\underline{\underline{J}} & =\lambda_{i} \mathbf{v}_{i}, \quad i=0, \ldots, \operatorname{dim}\{\mathbf{x}\}-1
\end{aligned}
$$

mit den Eigenwerten $\lambda_{i} \in \mathbb{C}$ und Eigenvektoren ${ }^{8} \mathbf{v}^{i} \in \mathbb{R}^{n}$. Sie liefert $n$-Richtungen $\mathbf{v}_{i}$, wobei der Realteil des zugehörigen Eigenwert $\lambda^{i}$ direkt die Größe der Expansion bzw. Kontraktion in dieser Richtung bestimmt. Im Folgenden ist immer der Realteil von $\lambda^{i}$ gemeint, wenn von Eigenwerten $\lambda^{i}$ gesprochen wird. Ein Problem dieser Zerlegung ist, daß im Allgemeinen die Eigenvektoren von $\underline{J}$ keine Orthonormalbasis bilden, weswegen wir einen Zustandsvektor $\mathbf{x}$ nicht einfach in die Eigenrichtungen zerlegen können $\mathbf{x} \neq \sum_{i=0}^{n-1}\left(\left\langle\mathbf{x} \mid \mathbf{v}^{i}\right\rangle \mathbf{v}^{i}\right)$. Dazu betrachten wir ein System (3.32) wo die Matrix $\underline{\underline{J}}$ vollen Rang besitzt und damit zwar die Eigenvektoren i.A. keine Orthonormalbasis bilden, aber immerhin eine Basis $\left\{\mathbf{v}^{0}, \ldots, \mathbf{v}^{n-1}\right\}$ definieren. Man bekommt nun eine eindeutige Zerlegung in der schiefen Basis $\left\{\mathbf{v}^{i}\right\}$ von $\mathbf{x}$ durch Lösen des linearen Gleichungssystemes $\left(\mathbf{v}^{0}, \ldots, \mathbf{v}^{n-1}\right) \mathbf{c}=\mathbf{x}$, wobei dann $c^{i}$ der Anteil von $\mathbf{x}$ am $i$-ten Eigenvektor $\mathbf{v}^{i}$ ist. Damit läßt sich $\mathbf{x}$ in der Eigenwertbasis durch $\mathbf{x}=\sum_{i=0}^{n-1} c^{i} \mathbf{v}^{i}$ eindeutig darstellen. Die expandierenden Richtungen des lokalen Flusses $\Phi_{\mathbf{x}}^{t}$ sind die Eigenvektoren $\mathbf{v}^{i}$ welche Eigenwerte $\lambda^{i}>1$ besitzen. Wir betrachten vorerst wieder ein dynamisches System mit einer instabilen Richtung $\mathbf{v}^{0}$. Das Kopplungsschema (3.24) kann nun wie folgt vollendet werden. Die Kopplungssignale ergeben sich nach Glg. (3.25) zu

$$
s_{\mathbf{x}}=c_{\mathbf{x}}^{0} \text { und } s_{\mathbf{y}}=c_{\mathbf{y}}^{0}
$$

Nun können wir den Teil des Abstandes zweier Trajektorien $\mathbf{x}_{\perp}=\mathbf{x}-\mathbf{y}$, der durch den Fluß expandiert wird, durch $\mathbf{x}_{\perp}^{e x p}=\left(s_{\mathbf{x}}-s_{\mathbf{y}}\right) \mathbf{v}_{\mathbf{y}}^{0}$ approximieren. Zur Ableitung der Kopplungsfunktion (3.26) betrachten wir die Wirkung von $\underline{\underline{J}}$ auf $\mathbf{x}_{\perp}^{\exp }$

$$
\underline{\underline{J}} \mathbf{x}_{\perp}^{e x p}=\underline{\underline{J}}\left(s_{\mathbf{x}}-s_{\mathbf{y}}\right) \mathbf{v}_{\mathbf{y}}^{0}=\lambda_{\mathbf{y}}^{0}\left(s_{\mathbf{x}}-s_{\mathbf{y}}\right) \mathbf{v}_{\mathbf{y}}^{0} .
$$

Damit erhalten wir als lokale Kopplungsfunktion (3.26)

$$
\begin{gathered}
\mathbf{C}_{\mathbf{x}}(\mathbf{x}, \mathbf{y})=\gamma_{\mathbf{x}}\left(s_{\mathbf{y}}-s_{\mathbf{x}}\right) \mathbf{v}_{\mathbf{x}}^{0} \\
\mathbf{C}_{\mathbf{y}}(\mathbf{x}, \mathbf{y})=\gamma_{\mathbf{y}}\left(s_{\mathbf{x}}-s_{\mathbf{y}}\right) \mathbf{v}_{\mathbf{y}}^{0}
\end{gathered}
$$

Die Kopplungsstärke $\gamma$ ist eindeutig durch den größten Eigenwert von $\underline{\underline{J}}(\mathbf{x})$ bestimmt. Bei unidirektionaler Kopplung ist $\gamma_{\mathbf{y}}=\lambda_{\mathbf{y}}^{0}$, während bei bidirektionaler Kopplung die

${ }^{8} \mathrm{Im}$ Allgemeinen wird eine Jordansche Normalform vorliegen, die der Einfachheit halber hier aber nicht behandelt werden soll. 
Kopplungsstärke noch halbiert wird $\gamma_{\mathbf{x}}=\frac{1}{2} \lambda_{\mathbf{x}}^{0}$ bzw. $\gamma_{\mathbf{y}}=\frac{1}{2} \lambda_{\mathbf{y}}^{0}$, da sonst die lokale Instabilität doppelt kompensiert würde und man möglicherweise über das Ziel hinausschießt. Die Anwendung dieser Kopplungsvariante wird in Abschn. 3.2.3 am Beispiel der Hénonabbildung demonstriert. Ein Problem an der Eigenwertzerlegung ist, daß auch komplexe Eigenwerte auftreten können, wodurch die Bestimmung der Kopplungskonstante $\gamma$ aus den lokalen Expansionsraten nicht ganz trivial wird. Für solche Fälle wird man deswegen auf eine andere Zerlegung zurückgreifen.

\section{Die Singulärwertzerlegung}

Zur Charakterisierung der lokalen Stabilitätseigenschaften des Flusses $\Phi^{t}$ hätten wir am liebsten eine Zerlegung, die

1. eine Orthonormalbasis $\mathbf{u}_{0}, \ldots, \mathbf{u}_{n-1}$ von $\underline{\underline{J}}$ liefert

2. mit reellen Expansionsraten $w^{0}>w^{1}>\cdots>w^{n-1} \in \mathbb{R}$.

Dies kann erfüllt werden, wenn man die Eigenwertzerlegung von $\underline{\underline{J}}^{t r} \underline{\underline{J}}$ betrachtet. Die Matrix $\underline{\underline{J}}^{t r} \underline{\underline{J}}$ ist positiv semidefinit, so daß ein Hauptachsentransformation eine Orthonormalbasis $\mathbf{v}_{0}, \ldots, \mathbf{v}_{n-1}$ mit Eigenwerten $\lambda_{0}>\lambda_{1}>\cdots>\lambda_{n-1}$ liefert. Das gleiche gilt für $\underline{\underline{J}} \underline{\underline{J}}^{t r}$. Dies führt auf die Singulärwertzerlegung(,,singular value decomposition $\left.(S V D)^{\text {“ }}\right)$ einer Matrix [66].

Definition 3.1 (Singulärwertzerlegung(SVD)) Sei $\underline{\underline{\mathbf{A}}}$ eine reelle $(n \times n)$-Matrix. Eine Zerlegung der Form $\underline{\underline{\mathbf{A}}}=\underline{\underline{\mathbf{U}}} \underline{\underline{\mathbf{W}}} \underline{\underline{\mathbf{V}}}^{\text {tr }}$, in der $\underline{\underline{\mathbf{U}}} \in \mathbb{R}^{(n, n)}$ und $\underline{\underline{\mathbf{V}}} \in \mathbb{R}^{(n, n)}$ orthogonale Matrizen und die $(n \times n)$-Matrix $\underline{\underline{\mathbf{W}}}=w^{i} \delta_{i j}$ eine Diagonalmatrix sind, heißt eine Singulärwertzerlegung von $\underline{\underline{\mathbf{A}}}$.

Die Singulärwertzerlegung existiert für jede reelle Matrix $\underline{\underline{\mathbf{A}}}$ und liefert $\operatorname{Rang}\{\underline{\underline{\mathbf{A}}}\}=r$ singuläre Werte größer Null und $n-r$ Singulärwerte $w^{i}=0$. Für unsere Zweck benutzten wir mit $\underline{\underline{\mathbf{U}}}=\left(\mathbf{u}^{0}, \ldots, \mathbf{u}^{n-1}\right), \quad \underline{\underline{\mathbf{V}}}=\left(\mathbf{v}_{0}, \ldots, \mathbf{v}_{n-1}\right)$ mit $\mathbf{u}^{i}, \mathbf{v}^{i} \in \mathbb{R}^{n}$ und $w^{0}>w^{1}>\cdots>$ $w^{n-1}$ die Singulärwertzerlegung von $\underline{\underline{J}}$ in der äquivalenten Form

$$
\underline{\underline{J}} \mathbf{v}^{i}=w^{i} \mathbf{u}^{i}, \quad i=0, \ldots, n-1 .
$$

Da $\left(\mathbf{v}^{0}, \ldots, \mathbf{v}^{n-1}\right)$ eine Orthonormalsystem bildet, können wir $\mathbf{x}$ in dieser Basis darstellen $\mathbf{x}=\sum_{i=0}^{n-1}\left(\left\langle\mathbf{v}^{i} \mid \mathbf{x}\right\rangle \mathbf{v}^{i}\right)$. Dann kann die lineare Abbildung von $\mathbf{x} \mapsto \underline{\underline{J}}(\mathbf{x}) \mathbf{x}$ durch

$$
\underline{\underline{J}} \mathbf{x}=\underline{\underline{J}} \sum_{i=0}^{n-1}\left(\left\langle\mathbf{v}^{i} \mid \mathbf{x}\right\rangle \mathbf{v}^{i}\right)=\sum_{i=0}^{n-1}\left(w^{i}\left\langle\mathbf{v}^{i} \mid \mathbf{x}\right\rangle \mathbf{u}^{i}\right)
$$

dargestellt werden. Wir identifizieren die $\mathbf{v}^{i}$ als instabile Richtungen des lokalen Flusses $\Phi^{t}(\mathbf{x})$, welche den Anteil des Zustand in den Richtungen $\mathbf{u}^{i}$ durch einen Faktor $w^{i}>1$ expandieren bzw. kontrahieren. Nun können wir das Kopplungsschema (3.24) vollständig 


\section{Die treibende Kraft}

fertigstellen. Wir betrachten zuerst wieder den Fall nur einer instabilen Richtung $\mathbf{v}^{0}$. Der Anteil von $\mathbf{x}_{\perp}=\mathbf{x}-\mathbf{y}$ an $\mathbf{v}_{\mathbf{y}}^{0}$ des $\mathbf{y}$-Systems ist

$$
\left\langle\mathbf{v}_{\mathbf{y}}^{0} \mid \mathbf{x}_{\perp}\right\rangle \mathbf{v}_{\mathbf{y}}^{0}=\left\langle\mathbf{v}_{\mathbf{y}}^{0} \mid(\mathbf{x}-\mathbf{y})\right\rangle \mathbf{v}_{\mathbf{y}}^{0} \approx\left(\left\langle\mathbf{v}_{\mathbf{x}}^{0} \mid \mathbf{x}\right\rangle-\left\langle\mathbf{v}_{\mathbf{y}}^{0} \mid \mathbf{y}\right\rangle\right) \mathbf{v}_{\mathbf{y}}^{0},
$$

damit ergeben sich

$$
s_{\mathbf{x}}=\left\langle\mathbf{v}_{\mathbf{x}}^{0} \mid \mathbf{x}\right\rangle \text { und } s_{\mathbf{y}}=\left\langle\mathbf{v}_{\mathbf{y}}^{0} \mid \mathbf{y}\right\rangle
$$

als Kopplungssignale nach Glg. (3.25). Zur Berechnung der Kopplungsfunktion (3.26) betrachten wir die Wirkung von $\underline{\underline{J}}$ auf $\mathbf{x}_{\perp}$ aus Glg. (3.37)

$$
\left.\underline{\underline{J}}\left\langle\mathbf{v}_{\mathbf{y}}^{0} \mid \mathbf{x}_{\perp}\right\rangle \mathbf{v}_{\mathbf{y}}^{0} \approx \underline{\underline{J}}\left(s_{\mathbf{x}}-s_{\mathbf{y}}\right) \mathbf{v}_{\mathbf{y}}^{0}\right)=w_{\mathbf{y}}^{0}\left(s_{\mathbf{x}}-s_{\mathbf{y}}\right) \mathbf{u}_{\mathbf{y}}^{0},
$$

welche die Expansion durch den Fluß approximiert. Damit berechnet sich die lokale Kopplungsfunktion (3.26) zu

$$
\mathbf{C}_{\mathbf{y}}(\mathbf{x}, \mathbf{y})=w_{\mathbf{y}}^{0}\left(s_{\mathbf{x}}-s_{\mathbf{y}}\right) \mathbf{u}_{\mathbf{y}}^{0} .
$$

Die Kopplungsstärke $\gamma=w(\mathbf{y})^{0}$ ist damit auch durch die lokalen Stabilitätsverhältnisse bestimmt.

Die Kopplungsfunktion $\mathbf{C}_{\mathbf{x}}(\mathbf{x}, \mathbf{y})$ für das $\mathbf{x}$-System bei bidirektionaler Kopplung entsteht durch Ersetzen von $\mathbf{y}$ durch $\mathbf{x}$. Ausserdem wird, wie bei der Eigenwertkopplung, die Kopplungsstärke $\gamma$ noch bei beiden Systemen halbiert.

In der Arbeit von Josic [52] wurde mathematisch bewiesen, daß lokal betrachtet und mit gewissen Vorraussetzungen, unidirektionale von bidirektionaler Kopplung nicht unterschieden werden kann und damit beide zumindest lokal äquivalent sind. Dies kann mit diesem Kopplungschema auch direkt gezeigt werden. Zum Erreichen von IS ist es egal ob man beide Systeme mit Kopplungsfunktionen (3.39) bzw. (3.34) mit Kopplungsstärke $\gamma=\frac{1}{2} w^{0}\left(\lambda^{0}\right)$ koppelt, oder ob man ein einzelnes System mit voller Kopplungsstärke antreibt. In jedem Fall verringern die Trajektorien beider Systeme um (im Idealfall exakt) den gleichen Betrag ihres gegenseitigen Abstandes. Damit macht es zum Erreichen von identischer Synchronisation mit diesem Kopplungsschema keinen Unterschied, ob unioder bidirektionale Kopplung verwendet wurde. Bei bidirektionaler Kopplung hat man sogar zum gleichen Zweck zwei Kopplungssignale $s_{\mathbf{x}}$ und $s_{\mathbf{y}}$ über einen Kanal zu übertragen, weswegen diese Kopplungsstrategie aus praktischen Gesichtspunkten eigentlich $\mathrm{zu}$ vermeiden ist.

Es soll hier nochmals auf den Unterschied zwischen gekoppelten iterierten Abbildungen und gewöhnlichen Differentialgleichungen bei der Anwendung der dynamischen Kopplung hingewiesen werden. Bei iterierten Abbildungen liegt die Flußabbildung $\Phi^{t}(\mathbf{x})$ explizit durch das Vektorfeld $\mathbf{f}$ vor, womit die obigen Ableitungen für die durchgeführten linearen Approximationen die optimale Kopplungsstrategie eindeutig festlegen. Bei gekoppelten ODEs ist die Flußabbildung $\Phi^{t}(\mathbf{x} 0)$ durch die Lösung der Integralgleichung $\int_{t=0}^{\infty} \mathbf{f}\left(\mathbf{x}_{0}\right) d t$ für alle möglichen Zustände $\mathbf{x}_{0} \in \mathbb{R}^{n}$ definiert und läßt sich i.A. nicht explizit angeben. f beschreibt dann das Geschwindigkeitsfeld des Flusses. Während bei iterierten Abbildungen der Zustand des getriebenen Systems durch Wirkung der lokalen 


\section{Die treibende Kraft}

Kopplung (im Idealfall) direkt auf den lokal transversal stabilen Teil von $\mathbf{x}_{\perp}$ korrigiert wird, kann bei DGL's nur die Richtung des Flusses verändert werden. Die dynamische Kopplung prägt im zeitkontinuierlichen Fall dem Geschwindigkeitsfeld eine neue Komponente auf, die das getriebene System in Richtung des transversal stabilen Teils von $\mathbf{x}_{\perp}$ führt, welcher aber nicht instantan erreicht werden kann. Dieser Fall wird gesondert in Abschn. 3.2.5 diskutiert werden.

Aus diesem Grund sind die oben abgeleiteten Kopplungsstärken $\gamma$ erstmal nur für den zeitdiskreten Fall gültig. Die dynamische Kopplung schaltet lokal die expandierende Wirkung des Flusses aus, so daß die stabilen Richtungen für die restliche Verkleinerung des Abstandes der Trajektorien sorgen können. Um dies deutlich zu machen, betrachten wir zwei unidirektional gekoppelte iterierte Abbildungen unter Wirkung der Eigenwertkopplung aus Glg. (3.34) und $\gamma_{\mathbf{y}}=\lambda_{\mathbf{y}}^{0}$

$$
\mathbf{y}^{n+1}=\mathbf{f}\left(\mathbf{y}^{n}\right)+\lambda_{\mathbf{y}^{n}}^{0}\left(s_{\mathbf{x}}-s_{\mathbf{y}}\right) \mathbf{v}_{\mathbf{y}}^{0} .
$$

OBdA initialisieren wir $\mathbf{x}^{n}$ und $\mathbf{y}^{n}$ derart, daß ihre Differenz $\mathbf{x}_{\perp}=\mathbf{y}-\mathbf{x}=\mathbf{v}_{\mathbf{y}}^{0}$ exakt in Richtung der größten Instabilität und damit auch der Kopplung zeigt. Die linearisierte Dynamik schreibt sich dann mit $\underline{\underline{J}}=\left.\frac{\partial \mathbf{f}}{\partial \mathbf{y}}\right|_{\mathbf{y}=\mathbf{x}}$ zu

$$
\mathbf{x}_{\perp}^{n+1}=\underline{\underline{J}} \mathbf{x}_{\perp}^{n}-\left(s_{\mathbf{y}}-s_{\mathbf{x}}\right) \lambda_{\mathbf{y}}^{0} \mathbf{x}_{\perp}^{n} .
$$

Da $s_{\mathbf{y}}-s_{\mathbf{x}}$ den Anteil von $\mathbf{x}_{\perp}=\mathbf{y}-\mathbf{x}$ an der instabilen Eigenrichtung $\mathbf{v}_{\mathbf{y}}^{0}$ darstellt, wird laut Vorraussetzung $s_{\mathbf{y}}-s_{\mathbf{x}}=\left\langle\mathbf{v}_{\mathbf{y}}^{0} \mid \mathbf{x}_{\perp}\right\rangle=\left\langle\mathbf{v}_{\mathbf{y}}^{0} \mid \mathbf{v}_{\mathbf{y}}^{0}\right\rangle=1$ und damit gilt

$$
\mathbf{x}_{\perp}^{n+1}=\underline{\underline{J}} \mathbf{x}_{\perp}^{n}-\lambda_{\mathbf{x}}^{0} \mathbf{x}_{\perp}^{n}=\underline{\underline{J}} \mathbf{x}_{\perp}^{n}-\underline{\underline{J}} \mathbf{x}_{\perp}^{n}=0 .
$$

Somit ist die instabile Richtung ausgeschaltet und der Fluß wirkt in erster Ordnung in dieser Richtung nicht mehr. Die Rechnung bleibt auch für beliebige Anfangsrichtungen von $\mathbf{x}_{\perp}$ gültig und kann auch analog für die SVD-Kopplungsmethode durchgeführt werden. Für beliebige benachbarte Anfangsbedingungen wird die Rechnung nur etwas umfangreicher und der entscheidende Punkt wird nicht ganz so klar deutlich.

\subsubsection{Anwendung am Hénonsystem}

Nun soll die dynamische Kopplungsmethode an der Hénonabbildung

$$
\tilde{\mathbf{x}}^{n+1}=\left(\begin{array}{c}
\tilde{x}^{n+1} \\
\tilde{y}^{n+1}
\end{array}\right)=\mathbf{f}\left(\tilde{\mathbf{x}}^{n}\right)=\left(\begin{array}{c}
1-a\left(\tilde{x}^{n}\right)^{2}+b \tilde{y}^{n} \\
\tilde{x}^{n}
\end{array}\right)
$$

ausführlich getestet werden. Für unsere Untersuchungen verwenden wir den Standardparametersatz $a=1.4, b=0.3$, welcher auf den Attraktor in Abb. 3.2a führt. Nicht alle Anfangsbedingungen $\mathbf{x} \in \mathbb{R}^{2}$ führen auf diesen Attraktor, sondern das System (3.40) besitzt bei dieser Parametrisierung einen zusätzlichen Fixpunkt im Unendlichen $\mathbf{x}=(-\infty,-\infty)$. Abb. 3.2b-c zeigt die Bassinstruktur von (3.40) in verschiedenen Vergrößerungsstufen. Anfangswerte in den weißen Flächen werden auf den chaotischen Attraktor in Abb. 3.2a 

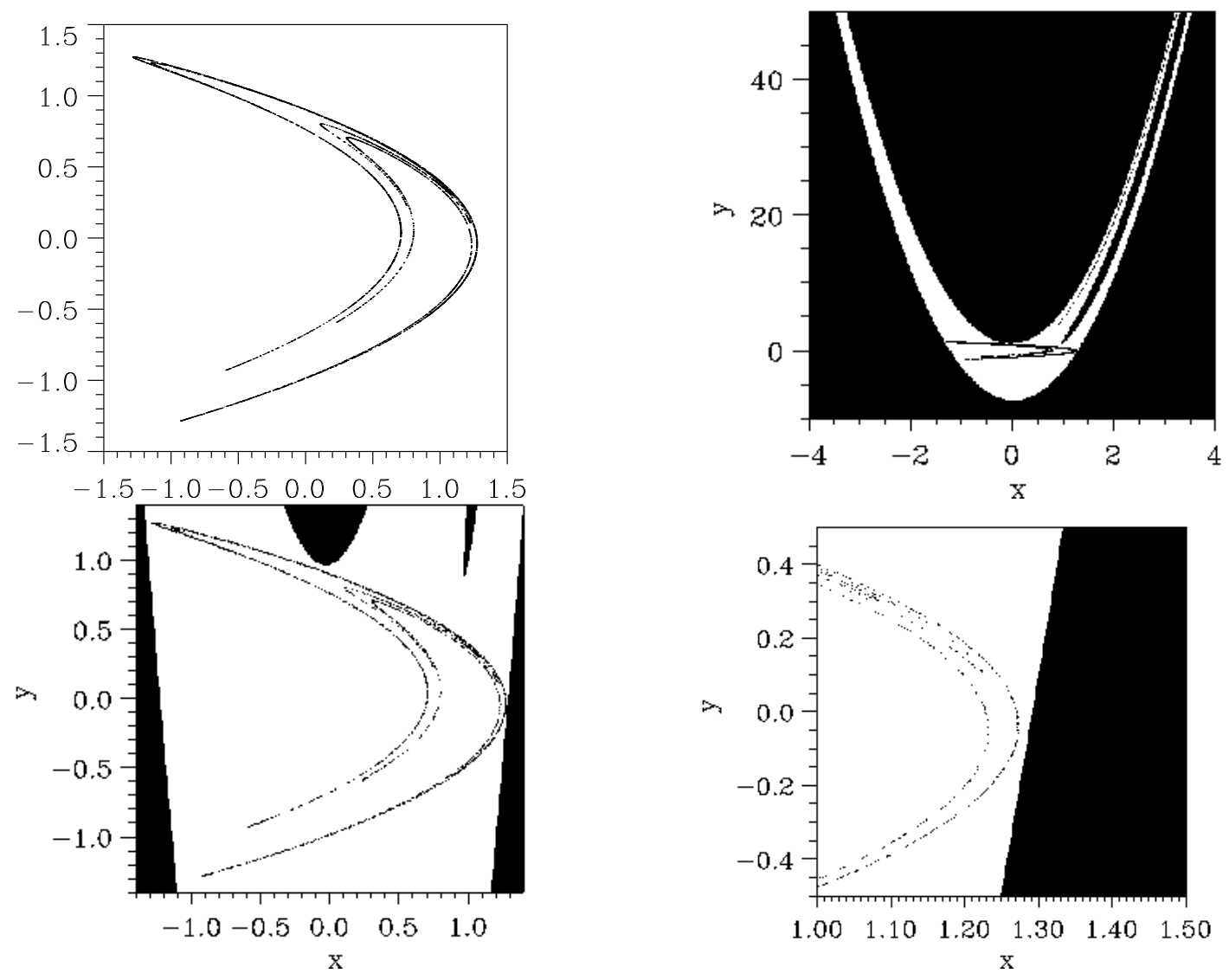

Abbildung 3.2.: a) Hénon Attraktor des Systems (3.40) mit $a=1.4, b=0.3$. Bassinstruktur des Henonsystem, wobei weiße Flächen zum chaotischen Attraktor a) führen und schwarze Flachen zum Fixpunkt im Unendlichen streben: b) Überblick der Bassinstruktur, c) detaillierter und d) Vergrößerung der rechten Bassingrenze.

abgebildet, während die schwarzen Flächen auf den Fixpunkt im Unendlichen führen. Abb. 3.2c offenbart, daß die Bassingrenzen dem Attraktor an manchen Stellen bedrohlich nahe kommen, welches in der Vergrößerung der rechten Bassingrenze in Abb. 3.2d besonders deutlich wird. Durch die Kopplungseingriffe in das getriebene System kann es an diesen Stellen passieren, daß das Bassin des chaotischen Attraktors verlassen wird und dadurch das System zu dem Fixpunkt im Unendlichen strebt. Aus diesem Grund wurde die Bassinstruktur auf hinreichend feinen Skalen berechnet und nur gekoppelt, falls dieser Eingriff nicht zum Verlassen des Bassins führen würde ${ }^{9}$. Neben den beschränk-

${ }^{9}$ Diese Fälle treten fast immer nur dann auf, wenn die beiden zu synchronisierenden Systeme noch relativ weit voneinander entfernt sind (Transientphase), so daß die Linearisierungen zur Berechnung der Eigenwert- bzw. SVD-Kopplung nur noch bedingt gültig sein werden. Die berechneten Kopplungs- 

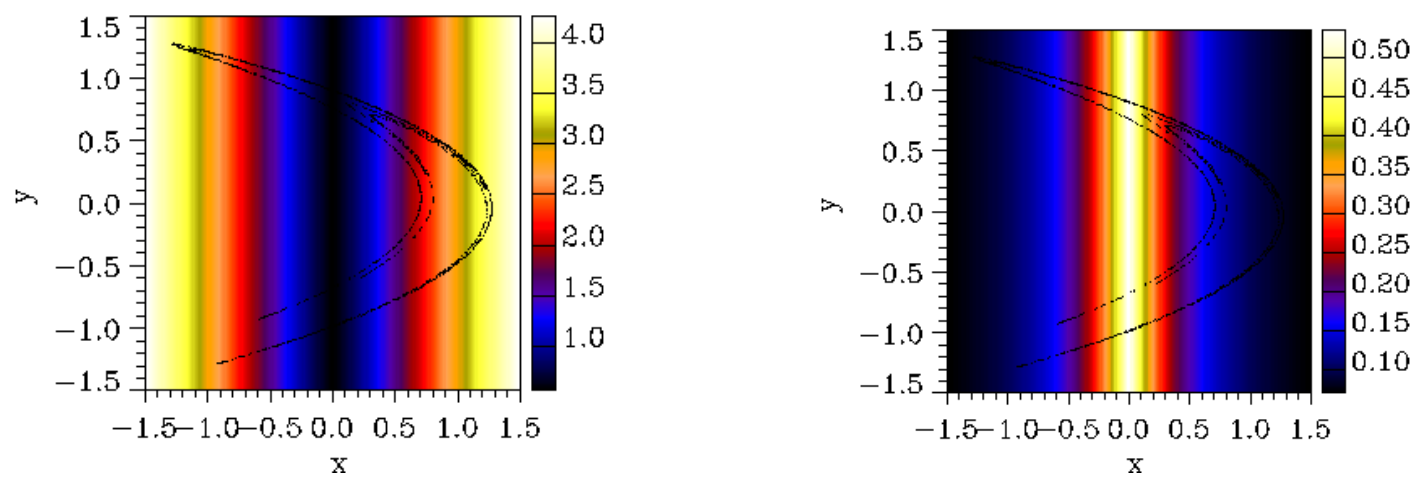

Abbildung 3.3.: Verteilung des Absolutbetrages der Eigenwerte auf dem Hénonattraktor. a) größter Eigenwert und b) kleinster Eigenwert.

ten Einzugsgebieten von Attraktoren existiert ein weiteres Phänomen, das gerade bei iterierten Abbildungen oft Probleme erzeugt. Dies sind Effekte, die auf der endlichen Rechengenauigkeit bei numerischen Simulationen basieren. Kürzlich wurden in [141] einige Resultate, wie z.B. Synchronisation bei positiven bedingten Lyapunovexponenten, als numerische Artefakte aufgrund endlicher Rechengenauigkeit entlarvt. Aus diesem Grund wurde in dieser Arbeit bei allen Simulationen ein schwaches weißes Rauschen von mindestens $\sim 10^{-12}$ zugefügt, wodurch bei der verwendeten numerischen Genauigkeit von $10^{-15}$ („double precision“) solche Artefakte ausgeschlossen werden.

Zur Bestimmung der Meß- und Kopplungsfunktionen der Eigenwertmethode berechnen wir aus der Jacobi-Matrix $\underline{\underline{J}}=\left(\begin{array}{cc}-2 a x & b \\ 1 & 0\end{array}\right)$ von Glg. (3.40) die Eigenwerte $\lambda_{0,1}=-a x \pm$ $\sqrt{b+(a x)^{2}}$ und die dazugehörigen Eigenvektoren $\mathbf{v}_{\lambda_{0,1}}=\left(\lambda_{0,1}^{2}+1\right)^{-\frac{1}{2}}\left(\begin{array}{c}\lambda_{0,1} \\ 1\end{array}\right)$. Abb. 3.3 zeigt die Verteilung des Absolutbetrages beider Eigenwerte auf dem Hénonattraktor. Wie erwartet, bewirkt der glatte Fluß f von (3.40) eine stetige Verteilung der Eigenwerte im Phasenraum. Die Eigenwerte hängen nur von der $x$-Komponente des Zustandes ab, so daß sich eine in $y$ homogene Verteilung ergibt, wobei die Instabilität der Zustände mit dem Betrag von $x^{n}$ zu den Rändern hin zunimmt. Zur Synchronisation zweier identischer Hénonsysteme (3.40), berechnen wir nun den Anteil $c^{0} \in \mathbb{R}$ von $\tilde{\mathbf{x}}^{n}$ an dem zu dem größten Eigenwert $\lambda^{0}\left(\tilde{\mathbf{x}}^{n}\right)$ gehörigen Eigenvektor, welcher nach Glg. (3.33) als Kopplungssignal $s_{\tilde{\mathbf{x}}}^{n}$ verwendet wird. Mit der entsprechenden Größe $s_{\mathbf{x}}^{n}$ vom x-System

eingriffe werden dann etwas ungenauer und können durchaus eine eng benachbarte Bassingrenze überschreiten. Ein andere Strategie wäre, solange zu warten, bis die zu synchronisierenden Systeme im Laufe der Zeitentwicklung zufällig einen gewissen Abstand unterschreiten, und dann erst die Kopplung einzuschalten. Wir haben uns zu der Überprüfung des Bassins entschlossen, da dies erstens nur ein Problem weniger Systeme ist und diese Fälle darüberhinaus auch nur relativ selten vorkommen. 
erhalten wir

$$
\mathbf{x}^{n+1}=\left(\begin{array}{l}
x^{n+1} \\
y^{n+1}
\end{array}\right)=\left(\begin{array}{c}
1-a\left(x^{n}\right)^{2}+b y^{n} \\
x^{n}
\end{array}\right)+\lambda^{0}\left(\mathbf{x}^{n}\right)\left(s_{\tilde{\mathbf{x}}^{n}}-s_{\mathbf{x}^{n}}\right) \mathbf{v}^{0}\left(\mathbf{x}^{n}\right)
$$

als getriebenes System.

Im Anschluß, wollen wir gleich auch den Fall der Kopplung mit singulären Werten behandeln. Die Singulärwertzerlegung von $\underline{\underline{J}}\left(\mathbf{x}^{n}\right)$ führt auf eine ähnliche Verteilung der singulären Werte $w^{0}$ und $w^{1}$ im Phasenraum wie die Eigenwertzerlegung von $\underline{\underline{J}}$. In Abb. 3.4
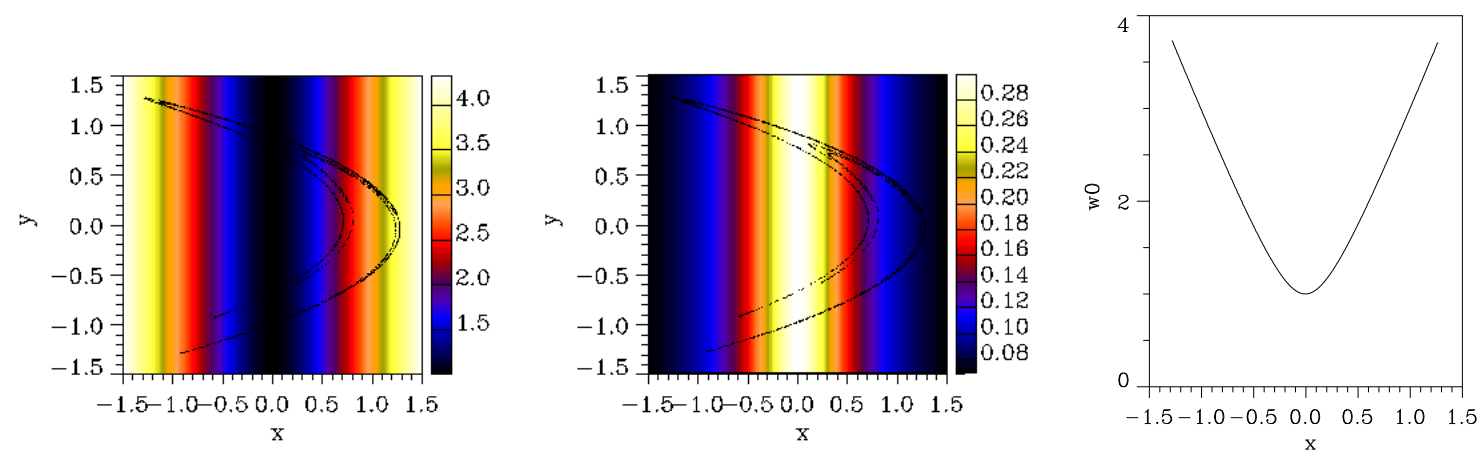

Abbildung 3.4.: Verteilung der Singulärwerte auf dem Hénonattraktor. a) größter Singulärwert, b) kleinster Singulärwert und c) größter Singulärwert gegen die $x$-Komponente vom Henon.

ist links die Verteilung des größten $w^{0}$ und in der Mitte des kleinsten $w^{1}$ Singulärwertes im Phasenraum gezeigt. Die Projektion von $w^{0}$ auf die $x$-Komponente im linken Bild von Abb. 3.4 zeigt deutlich, daß auch die singulären Werte ausschließlich von der $x$ Komponente der Hénonabbildung bestimmt werden. Mit der SVD $\underline{\underline{J}}(\tilde{\mathbf{x}}) \mathbf{v}_{\tilde{\mathbf{x}}}^{i}=w^{i} \mathbf{u}_{\tilde{\mathbf{x}}}^{i}$ von $\underline{\underline{J}}\left(\tilde{\mathbf{x}}^{n}\right)$ und $\underline{\underline{J}}\left(\mathbf{x}^{n}\right) \mathbf{v}_{\mathbf{x}}^{i}=w^{i} \mathbf{u}_{\mathbf{x}}^{i}$ von $\underline{\underline{J}}\left(\mathbf{x}^{n}\right)$ erhalten wir nach (3.38)

$$
s_{\tilde{\mathbf{x}}}^{n}=\left\langle\mathbf{v}_{\tilde{\mathbf{x}}}^{0} \mid \tilde{\mathbf{x}}^{n}\right\rangle
$$

als Kopplungssignal vom treibenden und $s_{\mathbf{x}}^{n}=\left\langle\mathbf{v}_{\mathbf{x}}^{0} \mid \mathbf{x}^{n}\right\rangle$ als Meßsignal vom getriebenen System. Das von $s_{\tilde{\mathbf{x}}}^{n}$ getriebene System

$$
\mathbf{x}^{n+1}=\left(\begin{array}{l}
x^{n+1} \\
y^{n+1}
\end{array}\right)=\left(\begin{array}{c}
1-a\left(x^{n}\right)^{2}+b y^{n} \\
x^{n}
\end{array}\right)+w^{0}\left(\mathbf{x}^{n}\right)\left(s_{\tilde{\mathbf{x}}}-s_{\mathbf{x}}\right) \mathbf{u}^{0}\left(\mathbf{x}^{n}\right)
$$

sollte nun mit (3.40) synchronisieren. In Abb. 3.5 werden die beiden Kopplungsvarianten direkt miteinander verglichen. Aufgetragen ist der Synchronisationsfehler $e=\|\tilde{\mathbf{x}}-\mathbf{x}\|$ gegen die Zeit. Die Kopplung mittels singulärer Werte (blaue durchgezogene Linie) führt schneller zur Synchronisation als die Eigenwertvariante (rote durchgezogene Linie) und scheint damit besser die lokalen Instabilitäten kompensieren zu können. In der Ableitung des Kopplungsschemas wurde der Anteil des Synchronisationsfehlers an der instabilen Richtung durch $\left.\left\langle\mathbf{v}_{\mathbf{x}}^{i} \mid(\tilde{\mathbf{x}}-\mathbf{x})\right\rangle \mathbf{v}_{\mathbf{x}}^{i} \approx\left(\left\langle\mathbf{v}_{\tilde{\mathbf{x}}}^{i} \mid \tilde{\mathbf{x}}\right\rangle-\left\langle\mathbf{v}_{\mathbf{x}}^{i}\right| \mathbf{x}\right)\right\rangle=s_{\tilde{\mathbf{x}}}-s_{\mathbf{x}}$ approximiert. Der 


\section{Die treibende Kraft}

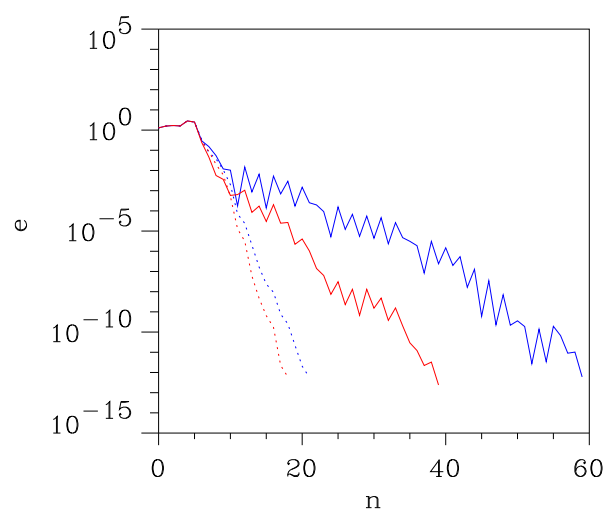

Abbildung 3.5.: Vergleich der Konvergenz zum synchronisiertem Zustand zwischen Eigenwert (blau) und SVD-Kopplung (rot). Die durchgezogenen Linien entsprechen der normalen Kopplung, während bei den gestrichelten Linien gemogelt wurde, indem das Kopplungssignal $s_{\tilde{\mathbf{x}}}$ mit den singulären Richtungen bzw. Eigenvektoren $\mathbf{v}_{\mathbf{x}}^{0}$ vom getriebenen System gebildet wurde.

Einfluß dieser Näherung auf das Synchronisationsschema kann getestet werden, indem zur Berechnung des treibenden Signals die instabilen Richtungen des getriebenen Systems verwendet werden. Da diese Information im treibenden System eigentlich nicht zur Verfügung steht, bezeichnen wir im Folgenden diese Variante etwas salopp als Mogelkopplung. Die gestrichelten Linien in Abb. 3.5 zeigen die Zeitentwicklung des Synchronisationsfehlers $e^{n}$ bei Verwendung der Mogelkopplung. Der Synchronisationsfehler fällt bei allen vier Kopplungsarten zuerst monoton auf $\approx 10^{-4} \mathrm{ab}$, konvergiert dann aber bei der realen Eigenwert- bzw. SVD-Kopplung etwas langsamer und leicht schwankend auf Null. Falls nun die Kopplungssignale ohne die Approximation berechnet werden, also die eigentlich nicht bekannten instabilen Richtungen im getriebenen System zur Berechnung des Kopplungssignales benutzt werden, fällt der Synchronisationsfehler weiter monoton auf Null ab. Das heißt, daß die gemachte Approximation $\mathbf{v}_{\tilde{\mathbf{x}}}^{i} \approx \mathbf{v}_{\mathbf{x}}^{i}$ bis ungefähr $10^{-4}$ praktisch exakt gültig ist, und danach leichte Abweichungen, z.B. in den instabile Richtungen $\mathbf{v}^{i}$, auftreten, welche dann zu dem nicht mehr streng monotonen Abfall des Synchronisationsfehlers führen. Insgesamt ist die gemachte Approximation aber durchaus gerechtfertigt und die etwas langsamere Konvergenz ist der Preis den man zur Übertragung eines nur skalaren Kopplungssignales bezahlen muß. Als nächstes untersuchen wir die Länge der Transiente zum synchronen Zustand für 50000 zufällig gewählte Anfangsbedingungen $\tilde{\mathbf{x}}_{0}, \mathbf{x}_{0} \in \mathcal{A}$ auf dem Hénonattraktor. Die Systeme gelten als synchronisiert falls der Synchronisationsfehler $e=\tilde{\mathbf{x}}-\mathbf{x}$ die Schranke $10^{-10}$ unterschreitet. In Abb. 3.6 ist das normierte Histogramm der Transientzeit $T$ bei Eigenwertkopplung (links) und Kopplung mit singulären Werten (rechts) aufgetragen. Daraus ergibt sich eine mittlere Transiente von 73 Iterationen bei der Eigenwertkopplung und von 44 Iterationen bei der SVD- 

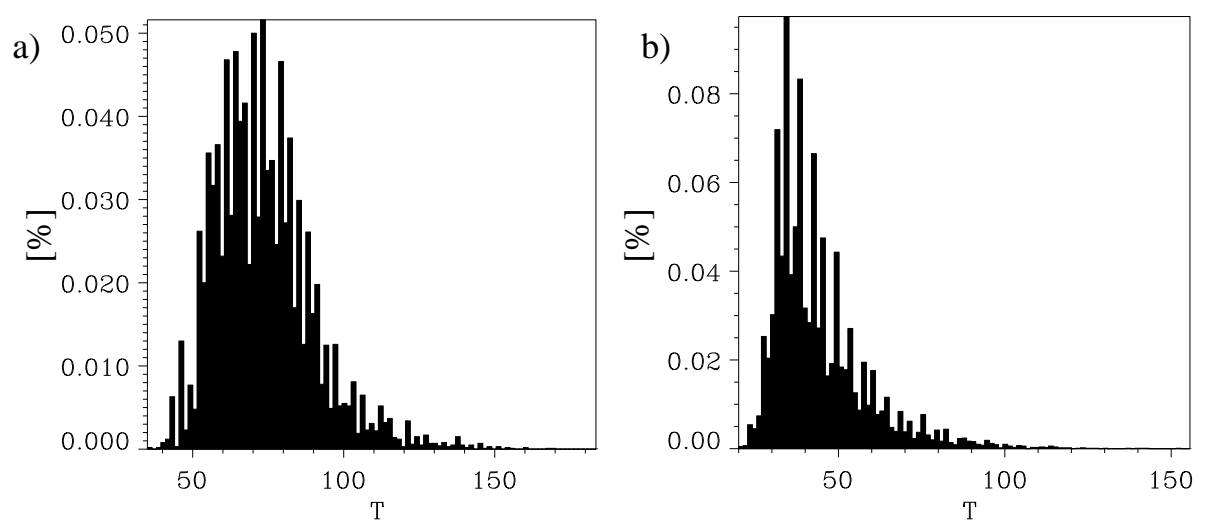

Abbildung 3.6.: Histogramme der Transientzeit bis zum synchronisierten Zustand von dynamischer Kopplung mittels a) Eigenwerten und b) mit der Singulärwertzerlegung.

Kopplung bei einer Standardabweichung von $\approx 15$ Iterationen bei beiden Histogrammen. Dies bestätigt den Eindruck von Abb. 3.5, daß die Singulärwertzerlegung die lokalen Stabilitätseigenschaften besser widerspiegelt als die Eigenwertzerlegung und zu einer praktisch doppelt so guten Performance führt. Um dies genauer zu quantifizieren berechnen wir direkt die lokalen Kontraktionsraten $\lambda_{l o k}^{n+1}=\log _{10}\left(\frac{e^{n+1}}{e^{n}}\right)=\log _{10}\left(\frac{\left\|\tilde{\mathbf{x}}^{n+1}-\mathbf{x}^{n+1}\right\|}{\left\|\tilde{\mathbf{x}}^{n}-\mathbf{x}^{n}\right\|}\right)$ auf den synchrononen Zustand. $\lambda_{\text {lok }}^{n}$ beschreibt um welchen Betrag der Synchronisationsfehler bei der letzten Iteration kontrahiert $\left(\lambda_{l o k}^{n}<0\right)$ bzw. expandiert $\left(\lambda_{l o k}^{n}>0\right)$ wurde. Mit dieser Größe konnen wir auch den größten bedingten Lyapunovexponenten des getriebenen Systems durch

$$
\lambda_{C}=\lim _{n \rightarrow \infty} \frac{1}{n} \sum_{n=0}^{n-1} \lambda_{l o k}^{n}
$$

definieren. Während $\lambda_{C}<0$ nur eine notwendige Bedingung (siehe Abschn. 2.1.1) für chaotische Synchronisation darstellt, kann mit $\lambda_{l o k}^{n}<0, \forall n$ global die Anziehung der Synchronisationsmannigfaltigkeit überprüft werden. Dazu haben wir die lokalen Kontraktionsraten $\lambda_{\text {lok }}^{n}$ entlang eine langen (100000 Iterationen) Trajektorie berechnet, wobei auf das treibende Signal $s_{\tilde{\mathbf{x}}}$ weißes Rauschen der Stärke $10^{-5}$ addiert wurde, und in Histogrammform in Abb. 3.7 aufgetragen. Die beiden linken Abbildungen zeigen die Kontraktionsraten für die Eigenwert-Kopplung und die beiden rechten die für die Kopplung mit singulären Werten. Oben wurden die realen Kopplungssignale verwendet wären unten wieder gemogelt wurde, indem das Kopplungssignal mit den instabilen Richtungen aus dem getriebenen System berechnet wurde. Der Schwerpunkt der Histogramme approximiert den bedingten (globalen) Lyapunovexponenten. Wir betrachten zuerst den Idealfall, wo die instabilen Richtungen des getriebenen Systems im treibenden bekannt sind, welcher im unteren Teil von Abb. 3.7 gezeigt ist. Es gilt $\lambda_{l o k}^{n}<0 \forall n$, d.h. für beide Kopplungsvarianten wird die Synchronisationsmannigfaltigkeit global anziehend, 

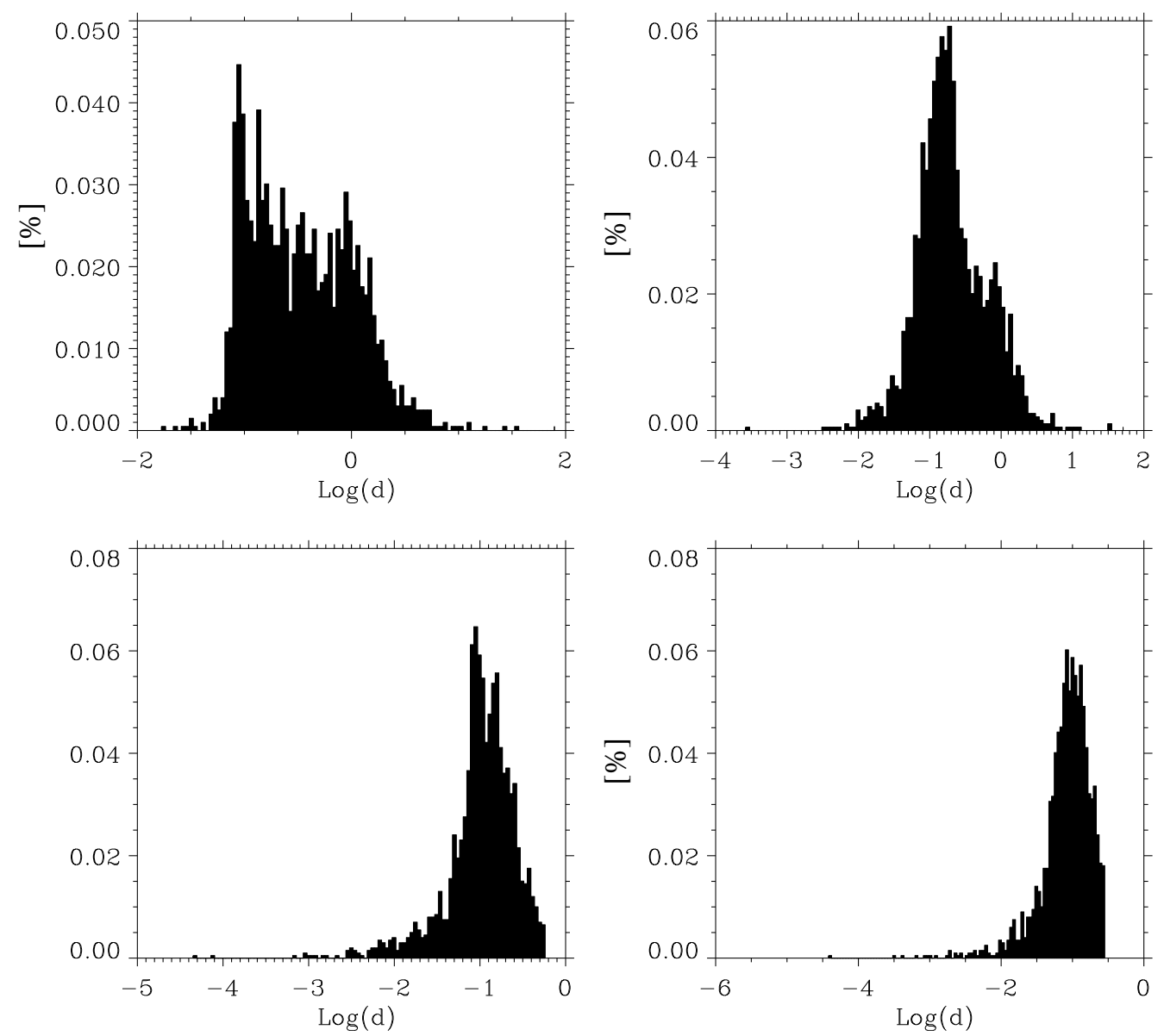

Abbildung 3.7.: Histogramme der Kontraktionsraten von der Eigenwert-Kopplung (links) und Kopplung mit der Singulärwertzerlegung (rechts). Oben: reale Kontraktionsraten und unten die mit der Mogelkopplung.

welches numerisch die oben gemachten Forderungen an die Kopplung bestätigt. Weiterhin zeigt sich auch hier, daß die SVD die instabilen Richtungen besser approximiert als die Eigenwertkopplung, welches sich durch den größeren bedingten Lyapunovexponenten(Schwerpunkt des Histogrammes) von $\approx-1.09$ gegenüber von $\approx-0.99$ bei der Eigenwertkopplung ausdrückt. Durch die schon öfters diskutierte Approximation der instabilen Richtungen des getriebenen Systems durch die des treibenden bewirkten Fehler, treten in Abb. 3.7 oben nun bei beiden Kopplungsarten auch lokale Expansionen des Synchronisationsfehlers $\lambda_{l o k}^{n}>0$ auf, welche aber nur einen kleinen Teil im Histogram darstellen. Dies stellt sich dann in einer Verbreiterung der Histogramme von $\lambda_{\text {lok }}$ und einem kleineren bedingten Lyapunovexponenten von $\approx-0.69$ bei der SVD-Kopplung und $\approx-0.47$ bei der Eigenwertkopplung dar.

Bei realen Systemen ist manchmal nicht jeder Punkt im Phasenraum einer Beeinflussung 
zugänglich oder man möchte so selten wie möglich steuernd in das System einwirken, wozu man dann die Information benötigt, an welchen Punkten der Steuereingriff am effektivsten wirksam ist. Die Idee der lokal dynamischen Kopplung ist, daß in jedem Punkt auf dem Attraktor $\mathcal{A}$ eine angepaßte (möglichst optimale) Kopplungsfunktion verwendet wird. Nachdem in Abschn. 3.1.4 gezeigt wurde, daß man nicht unbedingt zeitkontinuierlich koppeln muß um Synchronisation zu erreichen, stellt sich hier die Frage ob man im gesamten Phasenraum koppeln muß, oder ob eine Steuerung nur in einigen Bereichen des Zustandsraumes ausreichend zur Synchronisation ist. Dies ist in der Tat möglich, dazu wird man normalerweise das natürliche (invariante) $\operatorname{Maß} \mu(\mathcal{A})$ und darauf die instabilsten Punkte, z.B. charakterisiert durch den größten Singulärwert $w^{0}$, betrachten. Dies geschieht im nächsten Paragraphen, zuerst nutzen wir aus, daß die lokale Instabilität nur von der $x$-Komponente der Hénonabbildung abhängt, siehe auch Abb. 3.4c. In lin-
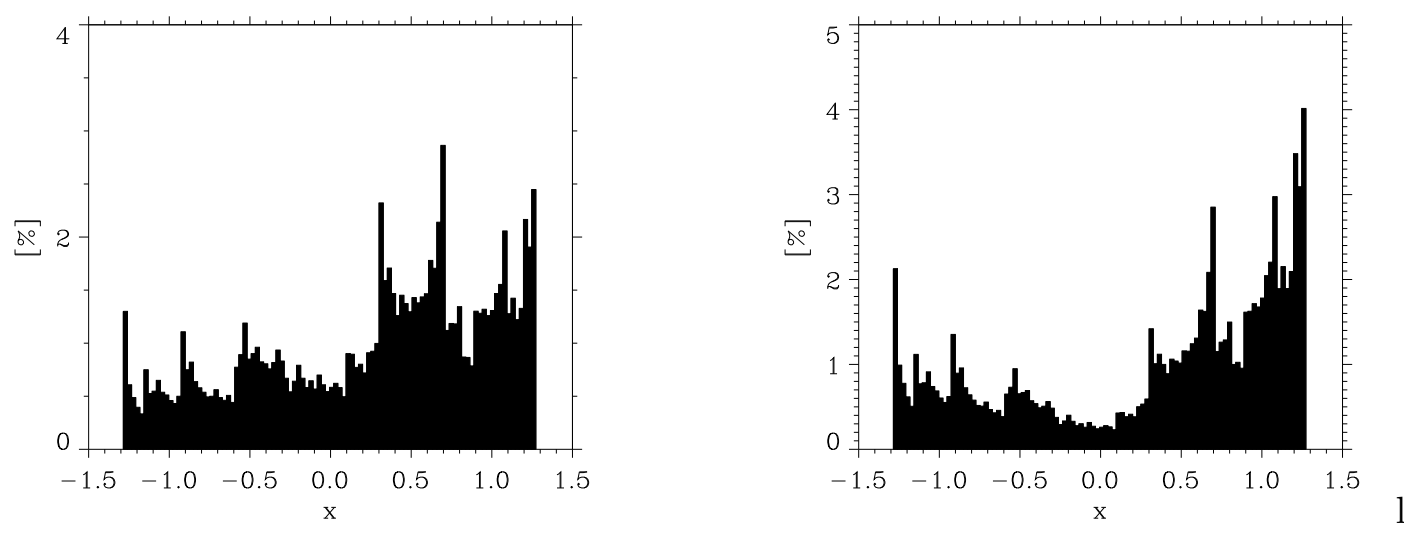

Abbildung 3.8.: Häufigkeitsverteilung der Punkte entlang der $x$-Komponente des Hénonattraktors (links) und zusätzlicher Gewichtung mit den Singulärwerten (rechts).

ken Teil von Abb. 3.8 ist die Häufigkeitsverteilung der Punkte entlang der $x$-Komponente des Hénonattraktors aufgetragen, welches eine Projektion des natürlichen Masses auf die $x$-Komponente darstellt. Danach hält sich eine Trajektorie bevorzugt im rechten Teil und am äußersten linken Abschnitt des Hénonattraktors auf. Die Verteilung im rechten Teil von Abb. 3.8 ist zusätzlich mit dem größten Singulärwert gewichtet, wodurch neben den Regionen, welche die Trajektorien am Häufigsten ansteuert, die Bereiche, welche den größten Anteil an der Desynchronisation haben, verstärkt berücksichtigt werden. An Abb. 3.8(rechts) wird deutlich, daß man um den Ursprung $x=0$ nicht koppeln muß, sondern hauptsächlich in den Bereichen $x<-1.0$ und $x>0.5$ steuernd eingreifen sollte. Wir haben uns für eine einfache Auswahl der zu koppelnden Regionen entschieden und koppeln mit der SVD-Methode nur falls $|x|>x_{c} \geq 0$ ist. Dadurch bleiben die Systeme in dem Streifen $-x_{c} \leq x \leq x_{c}$ ungekoppelt und wir koppeln nur in den Bereichen welche nach Abb. 3.4c auch für die größten Expansionen von $e^{n}$ verantwortlich sind. Da bei 
der Hénonabbildung mit dem verwendeten Parametersatz $w^{0}$ streng monoton von $|x|$ abhängt (Abb. 3.4c), kann diese Strategie auch anders formuliert werden. Es wird nur gekoppelt, falls der größte Singulärwert $w^{0}(\mathbf{x})$ größer als eine untere Schranke $w_{\text {min }}$ ist.
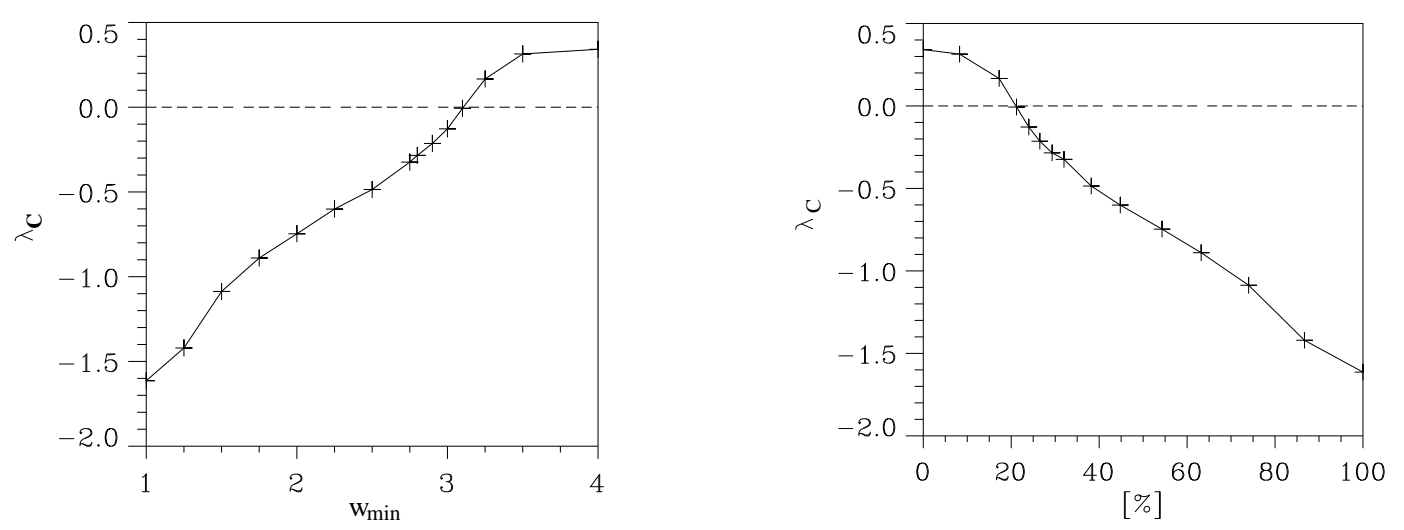

Abbildung 3.9.: Synchronisation mittels Singulärwertzerlegung, wobei nur gekoppelt wird, falls der aktuelle Singulärwert $w^{0}$ größer als eine untere Schranke $w_{\min }$ ist. Links ist der bedingte Lyapunovexponent $\lambda_{C}$ gegen $w_{\min }$ und rechts $\lambda_{C}$ gegen den Anteil der Kopplung gemessen an der Länge der Zeitreihe $L$ aufgetragen. Für $w_{\min } \leq 2.8$ (welches einem Anteil von $29.3 \%$ an der Zeitreihe entspricht) tritt intermittenzfreie Synchronisation auf.

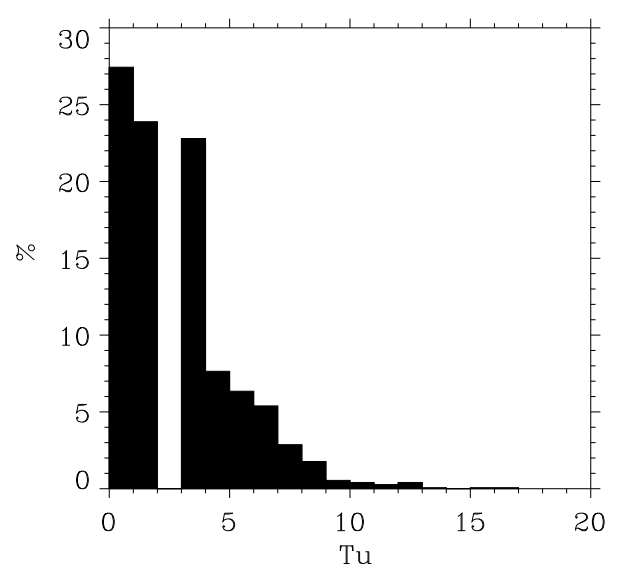

Abbildung 3.10.: Histogramm der kopplungsfreien Zeiten $T_{u}$ bei der SVD-Kopplung für $w_{\min }=2.8$.

Die entsprechenden Ergebnisse zu dieser Strategie sind in Abb. 3.9 dargestellt. Im linken 


\section{Die treibende Kraft}

Teilbild wurde der bedingte Lyapunovexponent $\lambda_{C}$ gegen die untere Kopplungsschranke $w_{\text {min }}$ aufgetragen, wobei $w_{\text {min }}=1$ den Grenzfall globaler Kopplung und $w_{\text {min }}=4$ keine Kopplung darstellt. Für $w_{\min }<3.1$ wird $\lambda_{C}<0$, so daß die Synchronisationsmannigfaltigkeit im Mittel anziehend wird. Diese Bedingung stellt sich hier aber ein weiteres mal als nur notwendig heraus und es wird zuerst Intermittenz beobachtet. Dies liegt daran, daß nur eine kleiner Teil des Phasenraumes gekoppelt ist und häufig die Zeitabstände zwischen zwei Kopplungseingriffen sehr groß werden. Für $w_{\min } \leq 2.8$ oder $x_{c}<1.0$ beobachten wir intermittenzfreie Synchronisation (innerhalb 50000 Iterationen der Abbildung). Mit Hilfe der Häufigkeitsverteilung von $x$ können wir $w_{\min }$ bzw. $x_{c}$ in den Anteil der Kopplungseingriffe an der Gesamtlänge der betrachten Zeitreihe umrechnen. Das rechte Teilbild von Abb. 3.9 zeigt $\lambda_{C}$ in Abhängigkeit des Prozentsatzes der Kopplungseingriffe an der Gesamtlänge $N$ der betrachteten Zeitreihe. Bei $w_{\min }=2.8$ werden 29,3\% der Punkte entlang der Trajektorie gekoppelt, so daß im Mittel nur bei 3 von 10 Iterationen der Hénonabbildung gekoppelt werden muß. Abbildung 3.10 zeigt die Häufigkeitsverteilung der Anzahl der Iterationen zwischen zwei Kopplungseingriffen $T_{u}$ bei $w_{\min }=2.8$. Die Trajektorie benötigt in seltenen Fällen durchaus 10 oder mehr Iterationen bis sie zu einem gekoppelten Teil des Phasenraumes zurückkehrt. In dieser Zeit können sich die Trajektorien wieder ungestört voneinander entfernen bevor die Kopplung diesem Trend entgegenwirken kann. Falls nun Trajektorien, z.B. schwach instabile periodische Orbits, existieren, welche gänzlich im ungekoppelten Teil des Phasenraumes liegen, können kurzzeitige Desynchronisationsereignisse nicht ausgeschlossen werden, so daß „high-quality"-Synchronisation bei dieser Strategie nicht garantiert werden kann. Falls man aber $w_{\min }$ weiter vermindert, verschwindet der Schwanz im Histogramm in Abb. 3.10 sehr schnell und die Synchronisation ist für endliche Zeiten sehr robust.

Das Konzept der zeitdiskontinuierlichen Kopplung („,sporadic driving“) aus Abschn. 3.1.4 ist natürlich auch bei der dynamischen Kopplung diskreter dynamischer Systeme anwendbar. Die lokale Kopplungsfunktion wird dabei alle $T$ Iterationen angewendet und die oben diskutierten Probleme mit langen ungekoppelten Zeitintervallen sind ausgeschlossen. Der bedingte Lyapunovexponente $\lambda_{C}$ bei „sporadic driving“ mittels Eigenwertkopplung (Sternchen) und SVD-Kopplung (Kreuze) ist in Abb. 3.11 gegen den Abstand T zwischen zwei Kopplungseingriffen aufgetragen. Intermittenzfreie Synchronisation wird bei Eigenwert-Kopplung für $T \leq 3$ und bei Singulärwert-Kopplung für $T \leq 5$ beobachtet. Bei der SVD-Kopplung muß damit nur jede 5'te Iteration gekoppelt werden, somit ist „sporadic driving“ effektiver als die oben diskutierte Kopplung bei der nur die instabilsten Bereiche $w^{0}>w_{\min }$ gekoppelt werden. Die dynamische Kopplung mittels singulärer Werte hat für alle $T$ den kleineren bed. Lyapunovexponenten $\lambda_{C}$ und benötigt weniger Kopplungssignale. Wie im gesamten Abschnitt stellt sich damit die Kopplung durch singuläre Werte als robuster heraus und bewirkt eine stärkere Anziehung auf den synchronen Zustand, weswegen wir im weiteren nur noch diese Kopplungsvariante untersuchen. Abb. 3.12 zeigt die Zeitentwicklung des Synchronisationsfehlers $e=\|\tilde{\mathbf{x}}-\mathbf{x}\|$ für "sporadic driving“ mit $T=7$ bei der SVD-Kopplung. Bei $T=7$ hat $\lambda_{C}$ gerade Null unterschritten und der synchrone Zustand sollte im Mittel anziehend sein. Beobachtet wird aber intermittente Synchronisation, wobei längere Zeiten scheinbar robuster Synchronisation $(n=0, \ldots, 1300)$ von ebenso langen Zeiten desynchronisierten Ver- 


\section{Die treibende Kraft}

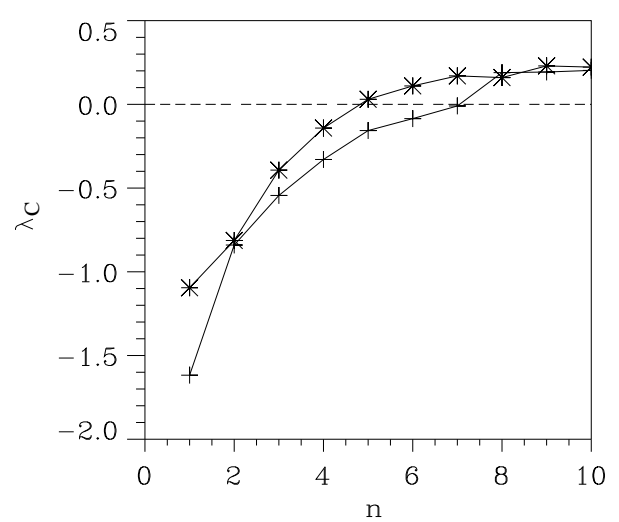

Abbildung 3.11.: Sporadic driving mittels Kopplung durch Eigenvektoren (Sternchen) und mittels SVD-Kopplung (Kreuze). Der bedingte Lyapunovexponent $\lambda_{C}$ ist gegen den Zeitabstand $n$ der Kopplungseingriffe geplottet. Intermittenzfreie Synchronisation tritt bei Eigenwert-Kopplung für $T \leq 3$ und bei Singulärwert-Kopplung für $T \leq 5$ auf.

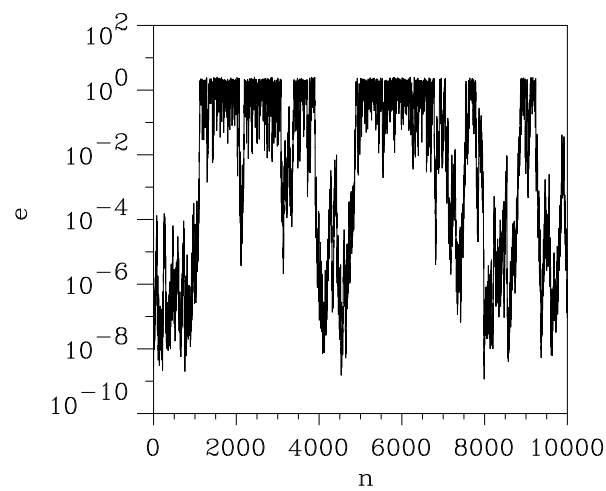

Abbildung 3.12.: Sporadic driving mittels Kopplung durch singuläre Werte. Der zeitliche Abstand zweier Kopplungseingriffe beträgt 7 Iterationen. Man beobachtet On-Off Intermittenz.

haltens unterbrochen ist. An diesem Beispiel wird besonders deutlich, wie wichtig bei numerischen Simulation die Addition von Rauschen kleiner Amplitude (hier $\approx 10^{-9}$ ) zur Vermeidung von numerischen Artefakten [141] ist. Der Synchronisationsfehler fällt einige Male herunter auf die Größenordnung des Rauschenstärke $\left(e^{n} \approx 10^{-9}\right)$ und ohne Rauschterm hätte man scheinbar robuste Synchronisation beobachtet, welche aber nur Folge endlicher Rechengenauigkeit wäre! 


\section{Die treibende Kraft}

\subsubsection{Partitionierung des Phasenraumes}

Bis jetzt mußte in jedem Zeitschritt und für alle Zustände die vollständige Kopplungsfunktion neu berechnet werden. Dies erfordert die Berechnung einer Singulärwert- oder Eigenvektorzerlegung, welche beide zwar standardmässig leicht bestimmbar sind, aber im Hinblick auf die einfache Struktur der Systemgleichungen, z.B. der Hénonabbildung (3.40), doch eine eher umfangreiche Rechnung erfordern. Unter praktischen Gesichtspunkten ist die dynamische Kopplung in der bisherigen Anwendung daher eigentlich als viel zu aufwendig anzusehen. Ein Ausweg liefert ein partitionierter Phasenraum. Für eine gegebene Partitionierung $\beta$ hätte man dann für jede Partition nur einmal die instabilen Richtungen zu bestimmen, welche dann die Kopplungsfunktion innerhalb der Partition eindeutig bestimmen. Da der Fluß eine glatte Funktion der Zustände ist, ändern sich die lokalen Stabilitätseigenschaften und damit die instabilen Richtungen nur wenig unter kleinen Zustandsänderungen, so daß keine beliebig feine Partitionierung notwendig sein sollte. Im Falle des Hénonattraktors ist es ausreichend das Quadrat $-1.5 \leq x^{n} \leq 1.5,-1.5 \leq y^{n} \leq 1.5$ im Phasenraum in $N * M$ Boxen $\mathrm{B}_{i j} ; i=1, \ldots, N, j=1, \ldots, M$ aufzuteilen. Damit erhalten wir die Partitionierung des Phasenraumes in paarweise disjunkte Boxen

$$
\beta=\left\{\mathrm{B}_{i j}\right\}_{i, j=1}^{N, M}, \quad \bigcup_{i, j=1}^{N, M} \mathrm{~B}_{i j} \subseteq \mathcal{B}, \quad \mathrm{B}_{i j} \cap \mathrm{B}_{i^{\prime} j^{\prime}}=0 \quad \text { für } \quad i \neq i^{\prime}, j \neq j^{\prime},
$$

welche den Attraktor mit dem Hauptteil seines Bassins $\mathcal{A} \subset \beta \subseteq \mathcal{B}$ enthält. In diesem Kapitel verwenden wir ausschließlich eine Parkettierung mit quadratischen Boxen, d.h. $N \times N$ Boxen der Größe $\frac{3.0}{N} \times \frac{3.0}{N}$ entlang der beiden Achsenrichtungen. Innerhalb der Partitionierung läßt sich nun das natürliche Maß $\mu$ durch

$$
\mu\left(\mathrm{B}_{i j}\right)=\lim _{T \rightarrow \infty} \frac{t_{\mathrm{B}_{i j}}}{T},
$$

approximieren. Dabei ist $t_{\mathrm{B}_{i j}}$ die Anzahl der Iterationen, während deren sich der Trajektorienabschnitt $\mathbf{f}^{t} \mathbf{x}_{0}^{n}$ in der Box $\mathrm{B}_{i j}$ aufhält. Für $N=M=100$ ist im linken Bild von Abb. 3.13 das natürliche Maß $\mu$ des Hénonattraktors $\mathcal{A}$ aus Glg. (3.40) dargestellt. Eine normale Trajektorie hält sich danach bevorzugt an den äußeren Teilen des Hénonattraktors $\mathcal{A}$ auf, wo auch die Abstoßung benachbarter Trajektorien am Größten ist. Bei dem rechten Teilbild von Abb. 3.13 wurde das natürliche Maß zusätzlich mit dem größten Singulärwert $w^{0}$ in der jeweiligen Box gewichtet. An diesem Bild wird deutlich, an welchen Stellen im Phasenraum die dynamische Kopplung den größten Effekt bewirkt. Der Fluß $\Phi^{t}$ des Hénonsystems wirkt in dem Streifen um $x^{n}=0$ kaum abstoßend, so daß man in diesem Bereich auch kaum steuernd eingereifen muß. Abb. 3.14 zeigt die Verteilung der singulären Richtungen $\mathbf{u}\left(\mathbf{x}^{n}\right)$ und $\mathbf{v}\left(\mathbf{x}^{n}\right)$ auf dem Hénonattraktor bei einer Partitionierung mit $20 \times 20$ Boxen, wobei die Richtungen den Mittelwert innerhalb der jeweiligen Box $B_{i j}$ darstellen. Wie die Singulärwerte in Abb. 3.4c, hängen auch $\mathbf{u}\left(\mathbf{x}^{n}\right)$ und $\mathbf{v}\left(\mathbf{x}^{n}\right)$ nicht von der $y$-Komponente des Hénonsystems ab. Zusätzlich sieht man die stetige Abhängigkeit der instabilen Richtungen von der $x$-Komponente, welche auch recht grobe Partitionierungen des Phasenraumes, von z.B. $N \leq 10$, für unsere 

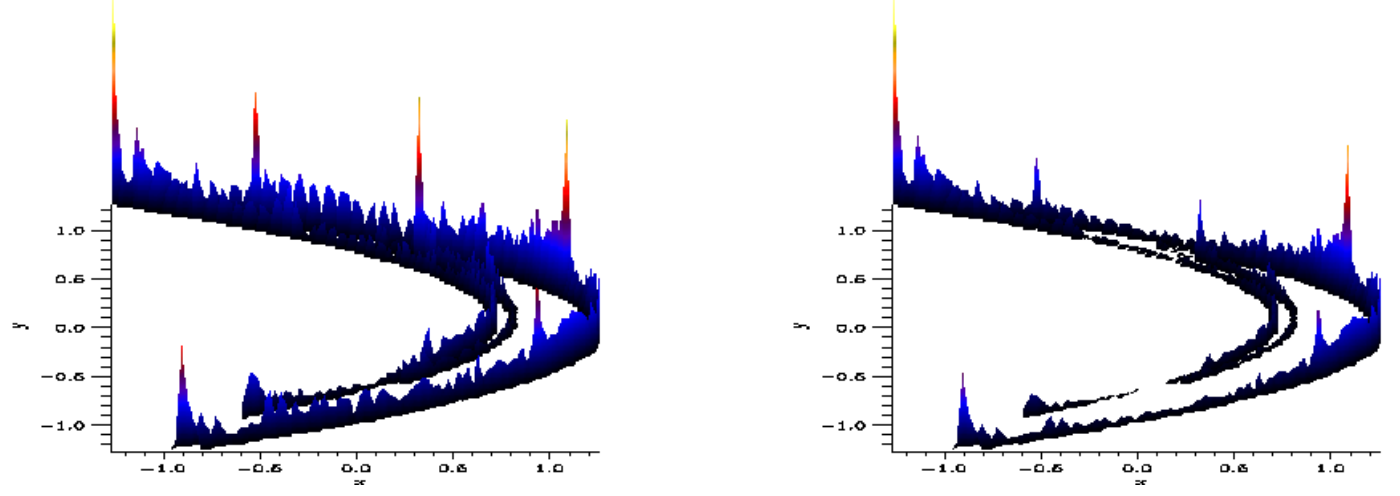

Abbildung 3.13.: Links ist das natürliche Maß $\mu$ vom Hénonattraktor $\mathcal{A}$ gezeigt, wobei der Phasenraum in $N=M=100 \times 100$ Boxen der Größe $\frac{3}{100}$ aufgeteilt wurde. Das rechte Bild zeigt das natürliche Maß $\mu_{w^{0}}$ gewichtet mit dem größten Singulärwert $w^{0}$ in den jeweiligen Boxen.
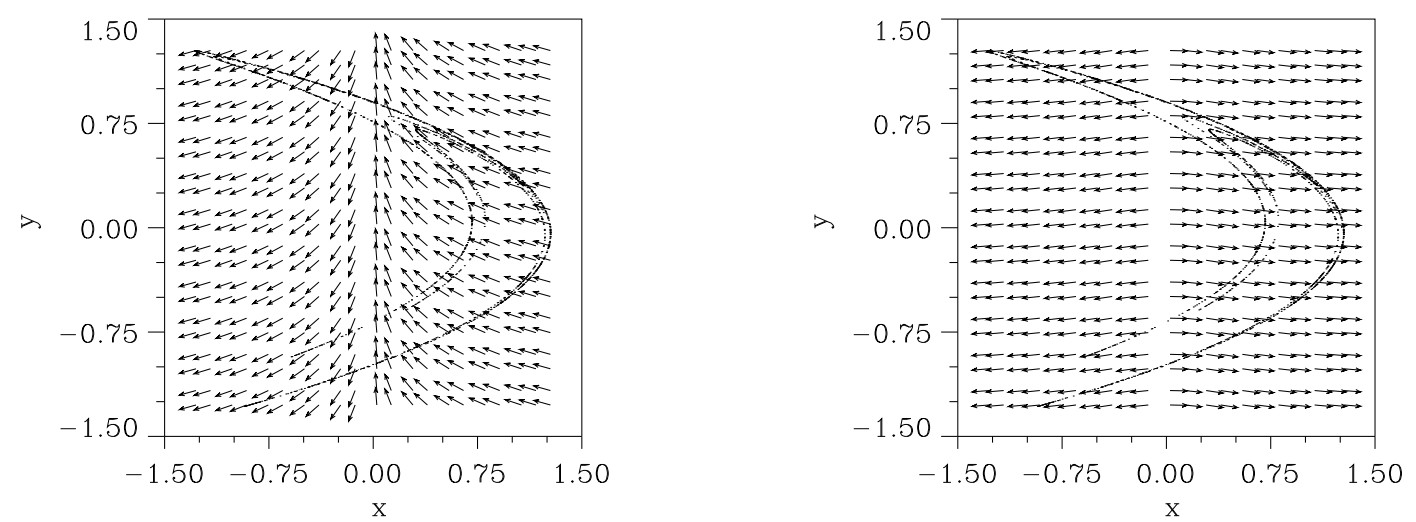

Abbildung 3.14.: Verteilung der singulären Richtungen der SVD korrespondierend zu dem größten Singulärwert in den jeweiligen Partitionen. Links ist $\mathbf{u}$ und rechts $\mathbf{v}$ aufgetragen.

Kopplungszwecke rechtfertigen. Falls man in allen Boxen des Phasenraumes koppelt, kann man die beiden Hénonsysteme noch mit einer $2 \times 2$-Partionierung synchronisieren, obwohl durch die Mittelung von $\mathbf{u}\left(\mathbf{x}^{n}\right)$ und $\mathbf{v}\left(\mathbf{x}^{n}\right)$ über die jeweilige Box, die lokalen Stabilitätseigenschaften nicht mehr gut approximiert werden können. Das dies bis hinunter auf $N=2$ trotzdem funktioniert, liegt daran, daß die singulären Richtungen auf dem Hénonattraktor nur schwach fluktuieren, siehe Abb. 3.14, und ist damit eine spezielle Eigenschaft dieses Systems. Bei komplexeren höherdimensionalen Systemen werden sicherlich etwas feinere Partitionen notwendig sein. 


\section{Die treibende Kraft}

Auch im partitionierten Phasenraum ist es nicht notwendig in allen Boxen $\mathrm{B}_{i j}$ zu kop-
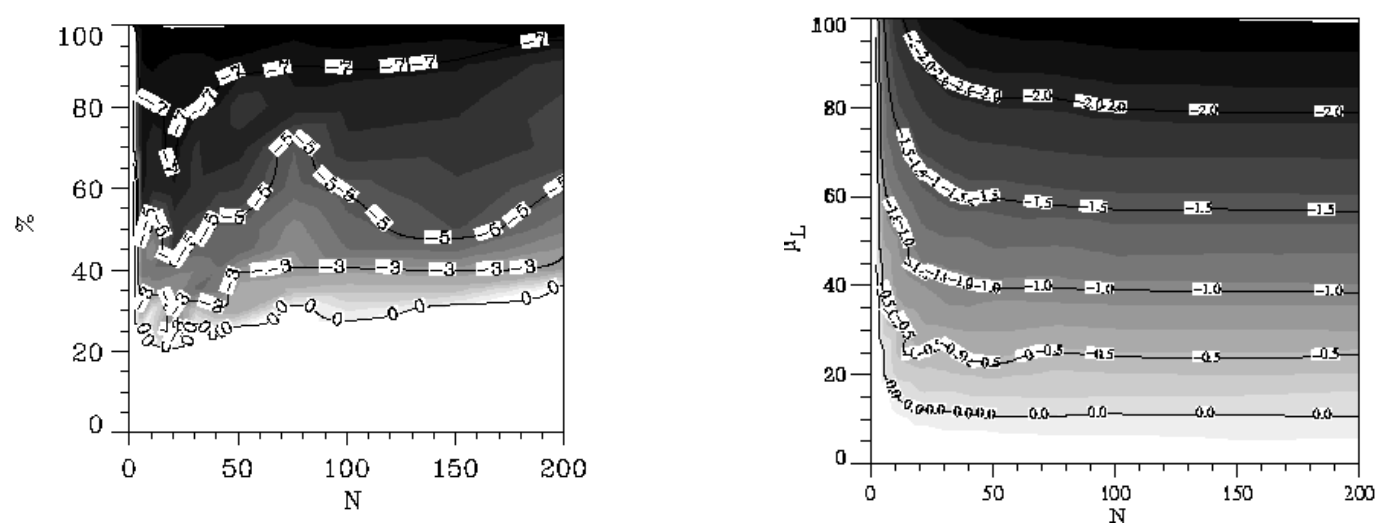

Abbildung 3.15.: Synchronisation mit der SVD-Kopplung im partitionierten Phasenraum, wobei die Kopplungspartitionen nach dem natürlichen Maß bestimmt wurden. Links ist der maximale Synchronisationsfehler $e$ farbkodiert und mit darübergezeichneten Contour-Linien, gegen den Prozentsatz $\mu_{L}$ der gekoppelten Bereiche (bezüglich des natürlichen Maßes auf $\mathcal{A}$ ) und gegen die Anzahl $N=M$ der verwendeten Boxen in jeder Achsenrichtung der Partitionierung geplottet. Im rechten Bild ist der bedingte Lyapunovexponent $\lambda_{C}$ gegen die gleichen Größen aufgetragen.

peln. Zur Auswahl der Partitionen in welcher die lokale Kopplungsfunktion angewendet wird, kann man das natürliche Maß aus Abb. 3.13a heranziehen. Um die Zeitabstände zwischen zwei Kopplungsereignissen nicht zu groß werden zu lassen, sollte man die Partitionen auswählen, die von einer typischen Trajektorie häufig besucht werden. Solche Partitionen zeichen sich durch große Werte des natürlichen Maßes in dieser Box $\mathrm{B}_{i j}$ aus. Zur Auswahl betrachten wir die Indexmenge $M=\left\{M_{1}, M_{2}, \ldots, M_{N * M}\right\}, \quad M_{k}=$ $(i, j), \quad i \in[1, \ldots, N], j \in[1, \ldots, M]$ welche bezüglich des natürlichen Maßes der Boxen $\mathrm{B}_{M_{k}}$ absteigend sortiert ist, d.h. es gilt $\mu\left(B_{M_{k}}\right) \geq \mu\left(B_{M_{k+1}}\right)$. Zur Kopplung verwenden wir nun die $L$ Boxen, welche von einer Trajektorie am Häufigsten besucht werden, d.h. $\left\{\mathrm{B}_{M_{k}}\right\}, \quad k=1, \ldots, L$. Damit liegt der Anteil $\mu_{L}=\sum_{k=1}^{L} \mu\left(B_{M_{k}}\right)$ einer typischen Trajektorie in Partitionen, wo die dynamische Kopplung wirksam ist, der Kehrwert dieser Größe $\frac{1}{\mu_{L}}$ ergibt den mittleren Abstand $T$ zwischen zwei Kopplungsereignissen. Das linke Bild von Abb. 3.15 zeigt den maximalen Synchronisationsfehler $e=\max \left\|\mathbf{x}^{n}-\tilde{\mathbf{x}}^{n}\right\|$ nach 50000 Iterationen unter Wirkung dieser Kopplungsstrategie in Abhängigkeit von $\mu_{L}$ und der Feinheit $N$ der benutzten Partitionierung. Im rechten Bild von Abb. 3.15 ist der bedingte Lyapunovexponent $\lambda_{C}$ gegen die gleichen Größen aufgetragen. Man muß damit im Mittel mindestens $\mu_{L} \approx 40 \%$ einer Trajektorie koppeln um intermittenzfreie Synchronisation $e<10^{-3}$ zu erhalten. Für diesen Wert werden Trajektorienabschnitte, welche sich lange Zeit außerhalb der gekoppelten Partitionen aufhalten (entsprechend Abb. 3.10), extrem unwahrscheinlich, so daß die gekoppelten Systeme nicht mehr genügend Zeit haben 
sich weit voneinander zu entfernen. Die Güte der Kopplung hängt bei diesem System nur schwach von der Feinheit $N$ der Partionierung ab, welches man aufgrund der recht homogenen Verteilung der singulären Richtungen auf dem Hénonattraktor auch erwartet. Im Bereich $12 \%<\mu_{L}<40 \%$ ist $\lambda_{C}$ zwar kleiner Null, es wird aber Intermittenz beobachtet, wobei die Länge der desynchronisierten Zeiten erwartungsgemäß mit steigenden Prozentsatz des gekoppelten Phasenraums $\mu$ abnehmen. In Abb. 3.16 wurde zur
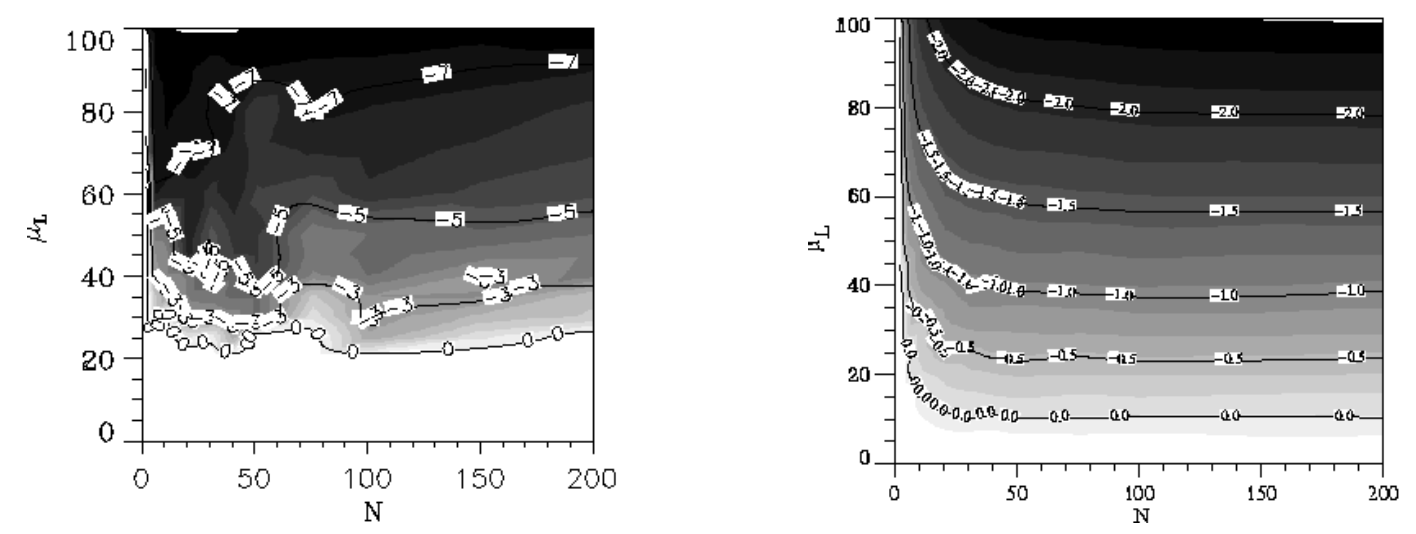

Abbildung 3.16.: Synchronisation mit der SVD-Kopplung im partitionierten Phasenraum, wobei jetzt die Kopplungspartitionen aus dem natürlichen Maß gewichtet mit dem größten Singulärwert bestimmt wurden. Links ist der maximale Synchronisationsfehler $e$ farbkodiert und mit darübergezeichneten Contour-Linien gegen den Anteil $\mu_{L}$ der gekoppelten Bereiche am natülichen Maß und gegen die Anzahl $N$ der Partitionen in jeder Achsenrichtung geplottet. Im rechten Bild ist der bedingte Lyapunovexponent $\lambda_{C}$ gegen die gleichen Größen aufgetragen.

Auswahl der Kopplungspartitionen das natürliche Maß $\mu_{w^{0}}$ gewichtet mit dem größten Singulärwert $w^{0}$ der jeweiligen Box aus Abb. 3.13b verwendet. Diese Kopplungsstrategie berücksichtigt, zusätzlich zur Frequentierung einer Box von der Trajektorie, die Stärke der Instabilität des synchronen Zustandes in dieser Partition. Abbildung 3.13b zeigt deutlich, daß durch diese Gewichtung der schwach instabile Streifen um $x^{n}=0$ zugunsten der stark abstoßenden Randgebiete stark an Gewicht verliert. Durch Berücksichtigung der lokalen Instabilität in der Auswahl der Kopplungspartitionen sollte man eine stabilere Synchronisation, insbesondere bei kleinen Anteilen $\mu_{L}$ der Kopplungsbereiche auf dem natürlichen Maß erhalten. In Abb. 3.16 wurde wieder $e$ und $\lambda_{C}$ gegen den Anteil der gekoppelten Bereiche am natürlichen Maß und gegen die Feinheit der Partitionierung $N$ geplottet. Falls wir wieder $e=10^{-3}$ als Grenze für stabile Synchronisation annehmen, dann bewirkt diese Auswahl der Kopplungspartitionen eine Verschiebung der Synchronisationsgrenze $e$ von $5 \%-10 \%$ nach unten, so daß jetzt weniger Kopplungspartitionen zur Erreichung des synchronen Zustandes notwendig sind. Im Gegensatz zur Kopplung in Abb. 3.15, wo das reine natürliche Maß zur Auswahl der gekoppelten Partitionen ver- 


\section{Die treibende Kraft}

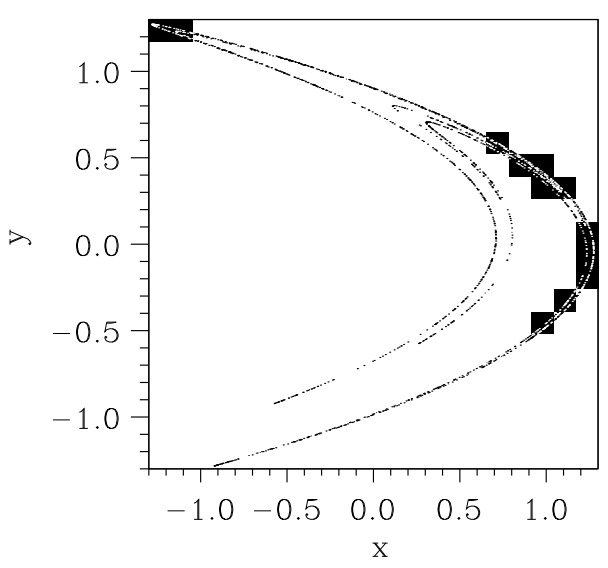

Abbildung 3.17.: Synchronisation mit der SVD-Kopplung im partitionierten Phasenraum bei einer Auflösung von $N=M=20$. Das Bild zeigt die 12 Partitionen welche zur Kopplung verwendet wurden, dies entspricht einen Anteil von $\mu \approx 30 \%$ der Kopplung auf dem natürlichen Maß.

wendet wurde, hat jetzt die Feinheit der Partitionierung $N$ einen etwas größeren Einfluß auf die minimale Anzahl der notwendigen Kopplungspartitionen. Eine grobe Partitionierung von $10<N<70$ Boxen in jeder Achsenrichtung scheint nun vorteilhafter zu sein. Dies liegt wahrscheinlich daran, daß man durch breitere Boxen eine größere Wahrscheinlichkeit hat, auch die benachbarten Trajektorien der Spitzen von Abb.3.13b in die Kopplung eher mit einzuschließen. Diese Trajektorien treten zwar nicht häufig auf, besitzen aber die gleiche transversale Instabilität und können gänzlich in ungekoppelten Bereichen verlaufen, so daß der Abstand zwischen zwei Kopplungsereignissen zu groß werden kann. Eine gröbere Partitionierung glättet das natürliche Maß etwas, welches in diesem Fall offensichtlich von Vorteil ist. Zur genaueren Untersuchung dieses Phänomenes würde man die Lage der instabilen periodischen Orbits auf dem Attraktor und insbesondere in den gekoppelten Partitionen betrachten, welches aber in diesem Kontext zu weit führt und deswegen in dieser Arbeit nicht untersucht wird. Für $N=M=20$ und 12 Kopplungspartitionen zeigt Abb. 3.17 die Lage der gekoppelten Bereiche auf dem Hénonattraktor. Die 12 Partitionen bedecken $\mu_{20} \approx 30 \%$ des natürlichen Maßes und deren Lage entspricht den größten Inhalten des gewichteten Maßes $\mu_{w^{0}}$. Wie erwartet bleibt die Mitte um $x^{n}=0$ ungekoppelt und die Randstreifen werden bevorzugt ausgewählt, wobei auffällig ist, daß nur die linke obere Ecke zur Kopplung verwendet wird. Der Grund dafür ist, daß sich eine Trajektorie, nach Abb. 3.13, für $x^{n}<0$ hauptsächlich dort aufhält und nur wenig Gewicht in den übrigen Partitionen der linken Seite besitzt. Im Abschn. 3.2.3 wurden zwei Hénonsysteme synchronisiert, indem nur die Bereiche auf dem Attraktor gekoppelt wurden, welche die größte lokale Instabilität besitzen. Übersetzt auf einen partitionierten Phasenraum werden jetzt nur die Partitionen zur Kopplung verwendet, deren mittlerer größter Singulärwert $w^{0}$ größer als eine vorgegebene 

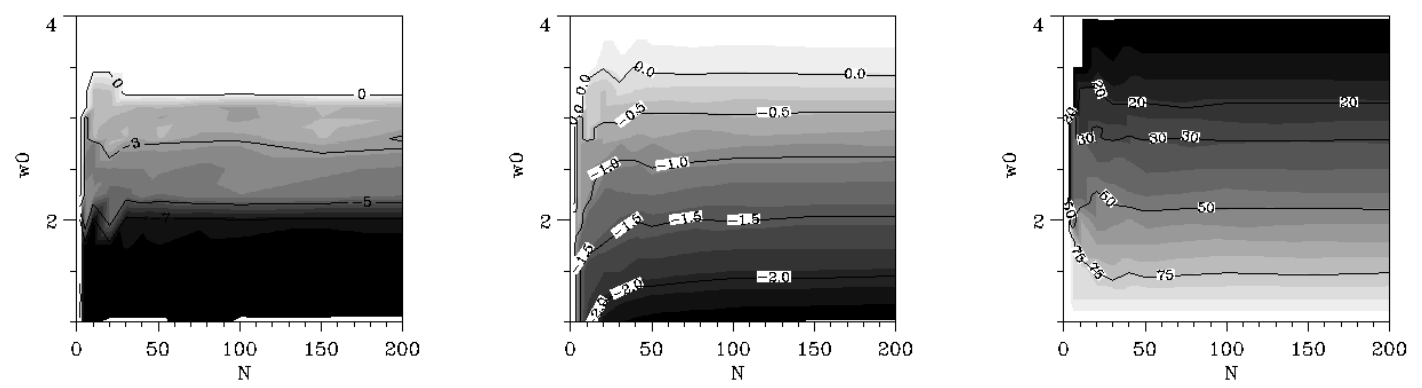

Abbildung 3.18.: Synchronisation mit der SVD-Kopplung im partitionierten Phasenraum wobei nur die Partitionen mit den größten Singulärwerten zur Kopplung herangezogen wurde. Links ist der maximale Synchronisationsfehler $e$ gegen den kleinsten Singulärwert $w_{\text {min }}$, ab dem die Kopplung aktiviert wurde und gegen die Feinheit der Partitionierung, gemessen durch die Anzahl $N$ der Partitionen in jeder Achsenrichtung, geplottet. Im mittleren Bild ist der bedingte Lyapunovexponent $\lambda_{C}$ und im rechten Bild ist der Prozentsatz der gekoppelten Bereiche am natürlichen Maß $\mu_{L}$ gegen die gleichen Größen aufgetragen.

untere Schranke $w_{\min }$ ist. Dies entspricht einer Kopplung ausschließlich in den beiden Randbereichen des Hénonattraktors. Abbildung 3.18 zeigt die Ergebnisse dieser Kopplungsstrategie. Im linken Bild ist der maximale Synchronisationsfehler $e$ gegen die untere Schranke $w_{\min }$ des Singulärwertes und gegen die Feinheit der Partitionierung geplottet. Das mittlere Bild zeigt den bedingten Lyapunovexponenten $\lambda_{C}$ und das rechte Bild den entsprechenden Anteil der gekoppelten Partitionen am natürlichen Maß in Abhängigkeit der gleichen Größen. Synchronisation tritt auf, falls alle Partitionen mit Singulärwert $w^{0}$ größer als $w_{\text {min }}=2.8$ zur Kopplung verwendet werden, welches einem Anteil von $30 \%$ am natürlichem Maß entspricht. Dies ist auch die untere Schranke bei unpartitionierten Phasenraum, so daß die Partitionierung keine Verschlechterung in der Synchronisation bei dieser Kopplungsstrategie bewirkt. Desweiteren ist die Kopplung der Partitionen mit den größten Singulärwerten unabhängig von der Feinheit $N$ der Partitionierung. Dies wird einsichtig, wenn man sich klarmacht, daß bei Variation von $N$ immer die gleichen Bereiche auf dem Hénonattraktor gekoppelt bleiben und sich nur die Boxgröße ändert. Bei den drei in diesem Abschnitt vorgestellten Kopplungsstrategien kann man für kleine Bedeckung $\mu$ des natürlichen Maßes wiederum nicht hundertprozentig garantieren, daß für alle Zeiten keine kurzzeitige intermittente Desynchronisation auftritt. Die Wahrscheinlichkeit, daß sich ein Trajektorienabschnitt lange Zeit in ungekoppelten Bereichen des Phasenraumes aufhält wird oberhalb der Synchronisationschwelle, die je nach Kopplungsstrategie bei $\mu>30 \%-40 \%$ liegt, zwar beliebig klein, aber nicht unbedingt Null. Um Synchronisation zu garantieren, muß man ein Maß basierend auf allen instabilen periodischen Orbits benutzen und zeigen, daß alle Orbits durch die Menge der gewählten 

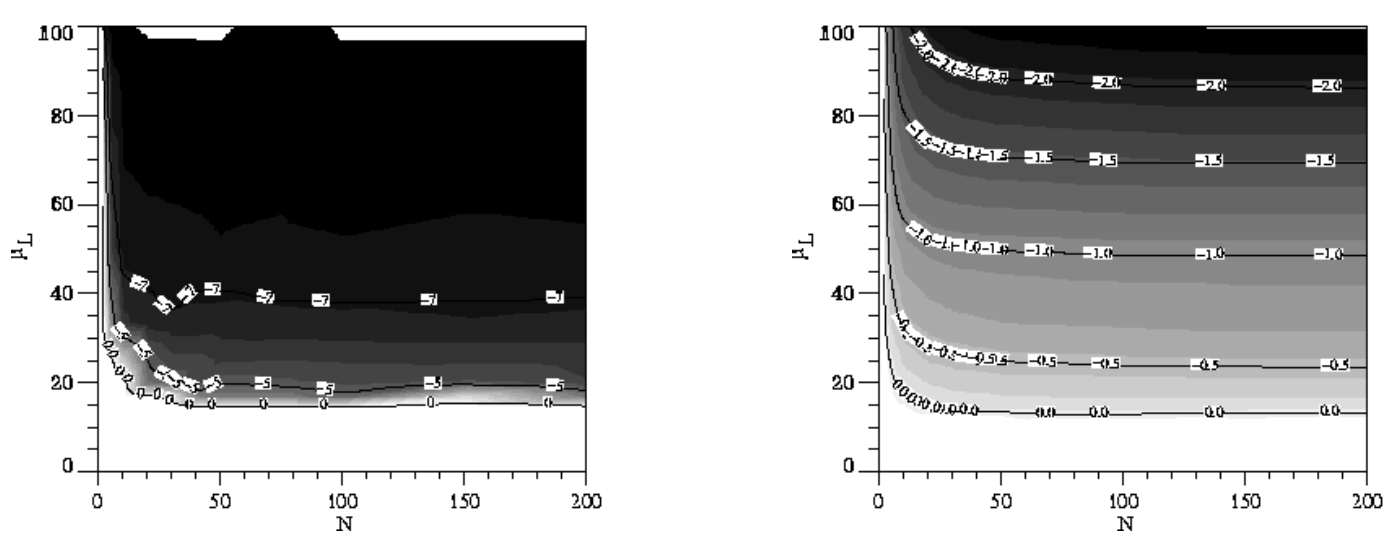

Abbildung 3.19.: Synchronisation mit der SVD-Kopplung im partitionierten Phasenraum wobei nur jedes T'te Signal zur Kopplung verwendet wurde (,sporadic driving"). Links ist der maximale Synchronisationsfehler $e$ farbkodiert und mit darübergezeichneten Contour-Linien gegen den Prozentsatz $\mu_{L}$ der gekoppelten Bereiche am natürlichen Maß und gegen die Feinheit der Partitionierung $N$ geplottet. Im rechten Bild ist der bedingte Lyapunovexponent $\lambda_{C}$ gegen die gleichen Größen aufgetragen.

Kopplungspartitionen miteinander synchronisieren. Dies wird i.A. schwierig zu zeigen sein und würde in diesem Kontext auch zu weit führen. Für die meisten praktischen Belange ist es aber ausreichend zu zeigen, daß der Synchronisationsfehler für sehr große Zeiten (in den Simulationen $N=50000-100000$ Iterationen) nach oben beschränkt bleibt.

Als letzte Kopplungsstrategie im partionierten Phasenraum wollen wir das „sporadic driving" anwenden, d.h. wir koppeln alle T-Iterationen, egal in welcher Partition sich das System gerade befindet, und sonst darf sich das getriebene System frei entwickeln. In Abb. 3.19 ist links der maximale Synchronisationsfehler $e$ und rechts der bedingte Lyapunovexponent $\lambda_{C}$ gegen $\mu_{L}$ und die Feinheit $N$ aufgetragen. Man muß mindestens alle 5-Iterationen $(\mu=20 \%)$ koppeln um intermittenzfreie Synchronisation zu erreichen, was identisch mit den Ergebnissen des unpartitionierten Phasenraumes ist. Da in diesem Fall eine Trajektorie nicht länger als $T$-Iterationen ungekoppelt ist, kann hier Intermittenz ausgeschlossen werden und "high quality synchronization“ kann für $T \leq 5$ garantiert werden. Diese Kopplungsvariante ist damit im Hinblick auf die zu übertragene Datenmenge der Kopplungssignale die Beste der bisher vorgestellten Strategien. Falls aber nicht alle Zustände im Phasenraum einer Messung bzw. Beeinflussung zugänglich sind, dann kann mit den anderen Kopplungsvarianten der gesamte Zustand aus räumlich lokal begrenzter Information rekonstruiert werden. 


\section{Die treibende Kraft}

\subsubsection{Dynamische Kopplung bei ODEs.}

In diesem Abschnitt wird demonstriert wie man das Konzept der dynamischen Kopplung auch auf zeitkontinuierliche Flüsse anwenden kann. Dazu betrachten wir zwei unidirektional gekoppelte ODEs

$$
\begin{aligned}
\dot{\mathbf{x}} & =\mathbf{f}(\mathbf{x}) \\
\dot{\mathbf{y}} & =\mathbf{f}(\mathbf{y})+\varepsilon\left(s_{x}-s_{y}\right) \mathbf{u}_{y}
\end{aligned}
$$

mit glatten Vektorfeld. Weiterhin soll der Fluß (zumindest im Mittel) nur eine instabile Richtung besitzen, so daß maximal ein positiver Lyapunovexponent vorliegt. Um die instabile Richtung $\mathbf{u}$ zu finden, benötigen wir die lokale Flußabbildung $\Phi^{\Delta t}(\mathbf{x})$, welche wir durch Diskretisierung von $\dot{\mathbf{x}}=\frac{\mathbf{x}(t+\Delta t)-\mathbf{x}(t)}{\Delta t}$ und anschließender Linearisierung um $\mathbf{x}(t)$ approximieren können:

$\mathbf{x}(t+\Delta t)=\mathbf{x}(t)+\Delta t \mathbf{f}(\mathbf{x}(t)) \approx \mathbf{x}(t)+\Delta t \underline{\underline{J}} \mathbf{x}(t)=(\underline{\underline{\mathbf{I}}}+\Delta t \underline{\underline{J}}) \mathbf{x}(t), \Delta t \ll 1$, wobei $\underline{\underline{\mathbf{I}}}$ die Einheitsmatrix und $\underline{\underline{J}}$ die Jacobimatrix $\frac{\partial \mathbf{f}}{\partial \mathbf{x}}$ im Punkt $\mathbf{x}(t)$ sind. Damit ist in einer kleinen Umgebung um $\mathbf{x}$ und für kurze Zeitabstände $\Delta t$ die Flußabbildung in guter Näherung durch die lineare Abbildung

$$
\underline{\underline{\mathbf{D} \Phi}}^{\Delta t}: \quad \mathbf{x}(t) \mapsto \mathbf{x}(t+\Delta t)=(\underline{\underline{\mathbf{I}}}+\Delta t \underline{\underline{J}}) \mathbf{x}=\underline{\underline{\mathbf{D} \Phi}}^{\Delta t} \mathbf{x}
$$

gegeben. Durch $\underline{\mathbf{D}}^{\Delta t}$ wird auch in guter Näherung die Zeitentwicklung des Abstandes $\mathbf{x}_{\perp}=\mathbf{x}-\mathbf{y}$ zweier eng benachbarter Trajektorien beschrieben. Die Singulärwertzerlegung von $\underline{\mathbf{D}}^{\Delta t}$ liefert nun analog zum zeitdiskreten Fall die instabile(n) Richtung(en) $\mathbf{u}, \mathbf{v}$, so daß der Zustand $\mathbf{y}$ nach dem Zeitschritt $\Delta t$ mittels

$$
\mathbf{y}(t+\Delta t) \mapsto \mathbf{y}(t+\Delta t)+w^{0}(t)\left\langle\mathbf{v}_{\mathbf{y}(t)}^{0} \mid \mathbf{x}_{\perp}(t)\right\rangle \mathbf{u}_{\mathbf{y}(t)}^{0}
$$

zu korrigieren ist, um die Expansion von $\mathbf{x}_{\perp}(t)$ durch den Fluß auszugleichen. Damit sollten sich nun zwei ODEs durch partielles Ersetzen („sporadic driving“) nach Glg. (3.49) alle $\Delta t$ Zeitschritte identisch synchronisieren lassen.

Einen Kopplungsterm für das Vektorfeld $\mathbf{f}$ von Glg. (3.47) erhält man durch folgenden Überlegung. Innerhalb der Linearisierung entfernen sich benachbarte Zustände exponentiell in der Zeit voneinander. Falls die Trajektorien zur Zeit $t=0$ einen Abstand von $\left\|\mathbf{x}_{\perp}(0)\right\|=1$ hatten, so haben sie nach $\Delta t$ Zeiteinheiten den Abstand $\left\|\mathbf{x}_{\perp}(\Delta t)\right\| \sim e^{\lambda \Delta t}$, wobei $\lambda$ die Divergenz quantifiziert (z.B. der Lyapunovexponent). Nach Glg. (3.49) gilt nach dem Zeitschritt $\Delta t$ für den expandierenden Anteil von $\mathbf{x}_{\perp}$

$$
\begin{aligned}
\left\|\left\langle\mathbf{v}^{0} \mid \mathbf{x}_{\perp}(t)\right\rangle\right\|=\left\|\left\langle\mathbf{v}^{0} \mid \mathbf{x}_{\perp}(t)\right\rangle \mathbf{v}^{0}\right\| & \mapsto\left\|\left\langle\mathbf{v}^{0} \mid \mathbf{x}_{\perp}(t)\right\rangle \underline{\underline{\mathbf{D} \Phi}}^{\Delta t} \mathbf{v}^{0}\right\|=\left\|\left\langle\mathbf{v}^{0} \mid \mathbf{x}_{\perp}(t)\right\rangle w^{0} \mathbf{u}_{\mathbf{y}(t)}^{0}\right\| \\
& =\left\|\left\langle\mathbf{v}^{0} \mid \mathbf{x}_{\perp}(t)\right\rangle\right\| w^{0},
\end{aligned}
$$

mit $\|\mathbf{u}\|=1$. Im zeitkontinuierlichen Fall wird der instabile Anteil von $\mathbf{x}_{\perp}(t)$ in der Zeit $\Delta t$ nach $\left\|\left\langle\mathbf{v}^{0} \mid \mathbf{x}_{\perp}(t)\right\rangle\right\| \mapsto e^{\lambda \Delta t}\left\|\left\langle\mathbf{v}^{0} \mid \mathbf{x}_{\perp}(t)\right\rangle\right\|$ expandiert, so daß durch Vergleich der Singulärwert $w^{0}$ direkt mit $w^{0}=e^{\lambda \Delta t}$ identifiziert werden kann. Um die im System innewohnende Divergenz $\lambda$ benachbarter Trajektorien (linear) zu kompensieren muß daher im Geschwindigkeitsfeld $\mathbf{f}$ eine Strömung der Stärke $\varepsilon=\ln \left(w^{0}\right) / \Delta t$ entgegen der 


\section{Die treibende Kraft}

Richtung der Instabilität $\mathbf{u}_{\mathbf{y}}^{0}$ induziert werden. Damit erhält man für die dynamische Kopplung bei ODEs den Kopplungsterm

$$
\mathbf{C}(\mathbf{x}, \mathbf{y})=\gamma\left(s_{x}-s_{y}\right) \varepsilon \mathbf{u}_{\mathbf{y}}, \text { mit } s_{x}=\left\langle\mathbf{v}_{x} \mid \mathbf{x}\right\rangle, s_{y}=\left\langle\mathbf{v}_{y} \mid \mathbf{y}\right\rangle \text { und } \varepsilon=\frac{\ln \left(w_{y}^{0}\right)}{\Delta t},
$$

wobei die Vektoren $\mathbf{u}, \mathbf{v}$ und der Singulärwert $w^{0}$ nach jedem Zeitschritt $\Delta t$ aktualisiert werden müssen ${ }^{10}$. Mit dem zusätzlichen Kopplungsgewicht $\gamma$ kann der Grad der Dissipation zum synchronen Zustand noch weiter verstärkt werden.

In Anhang A wird der Grenzübergang von $\Delta t \rightarrow 0$ vollzogen, welcher auf die exakten Kopplungsterme in Glg. (3.51) führt. Das Bemerkenswerte ist, daß alle Information aus der Jacobimatrix des Vektorfeldes $\underline{\underline{J}}=\frac{\partial \mathbf{f}}{\partial \mathbf{x}}$ am Punkt $\mathbf{x}$ berechnet werden kann, so daß nun nicht mehr der lokale Fluß $\Phi^{\Delta t}$ approximiert werden braucht! Damit ergibt sich die Kopplungsfunktion

$$
\mathbf{C}(\mathbf{x}, \mathbf{y})=\gamma\left(s_{x}-s_{y}\right) \varepsilon \mathbf{v}_{\mathbf{y}}, \text { mit } s_{x}=\left\langle\mathbf{v}_{x} \mid \mathbf{x}\right\rangle, s_{y}=\left\langle\mathbf{v}_{y} \mid \mathbf{y}\right\rangle \text { und } \varepsilon=\frac{\mu^{0}}{2},
$$

welche die lineare Instabilität im Vektorfeld $\mathbf{f}$ exakt kompensiert, wobei $\mathbf{v}$ der Eigenvektor zum (reellen) größten Eigenwert $\mu^{0}$ von der symmetrischen Matrix $\underline{\underline{\mathbf{B}}}=\underline{\underline{J}}^{t r}+\underline{J}$ ist. Falls der Fluß im Punkt $\mathbf{x}$ lokal stabil ist, d.h. $\mu^{0}<0 \Rightarrow \varepsilon<0$, braucht überhaupt nicht gekoppelt zu werden, so in diesen Punkten $\varepsilon=0$ gesetzt wird. Tatsächlich darf in diesen Fällen der Kopplungsterm aus Glg. (3.51) bzw. Glg. (3.50) nicht angewandt werden, da er immer entgegen der größten expandierenden Richtung wirkt, welche hier aber kontrahierend wirkt, so daß die Kopplung in diesen Bereichen den synchronen Zustand destabilisiert. Ein Ausweg ist $\varepsilon=\left|\mu^{0}\right| / 2$ zu verwenden, so daß die (schwach) kontrahierende Richtung des Flusses weiter lokal stabilisiert wird. Andererseits ist das System in diesen Punkten transversal stabil, so daß für eine minimale Kopplungsstrategie keine Beeinflussung stattfinden sollte. In dieser Arbeit folgen wir der letzteren Philosophie, so daß nur Instabilitäten des Flusses entgegengewirkt wird und stabile Bereiche unbeeinflußt bleiben.

Analog zum zeitdiskreten Fall kann man durch Partitionierung des Phasenraumes und anschließender Mittelung von $\mathbf{v}$ und $\varepsilon$ aus $\underline{\mathbf{B}}=\underline{J}^{t r}+\underline{J}$ in jeder Box, die dynamischen Kopplungsfunktionen fest angeben, womit die Kopplung dann auch in Echtzeit realisierbar sein sollte. Bei bidirektionaler Kopplung kann das Kopplungsgewicht $\varepsilon$ wieder halbiert werden.

Als Beispiel betrachten wir zwei unidirektional gekoppelte Lorenzoszillatoren:

$$
\begin{array}{ll}
\dot{x_{1}}=10\left(x_{2}-x_{1}\right) & \dot{y_{1}}=10\left(y_{2}-y_{1}\right) \\
\dot{x_{2}}=\left(28-x_{3}\right) x_{1}-x_{2} & \dot{y_{2}}=\left(28-y_{3}\right) y_{1}-y_{2} \\
\dot{x_{3}}=x_{1} x_{2}-\frac{8}{3} x_{3} & \dot{y_{3}}=y_{1} y_{2}-\frac{8}{3} y_{3}
\end{array}+\gamma\left(s_{x}-s_{y}\right) \varepsilon\left(\begin{array}{l}
v_{1} \\
v_{2} \\
v_{3}
\end{array}\right)
$$

\footnotetext{
${ }^{10}$ Die singulären Vektoren $\mathbf{u}$ und $\mathbf{v}$ zeigen für kleine $\Delta t \ll 1$ fast in die gleiche Richtung. Der kleine Unterschied rührt von der Richtungsänderung des abstoßenden Teiles des Flusses im Zeitschritt $\Delta t$ her, welcher in $\mathbf{u}$ berücksichtigt ist, während v nur die aktuelle Expansionsrichtung angibt.
} 


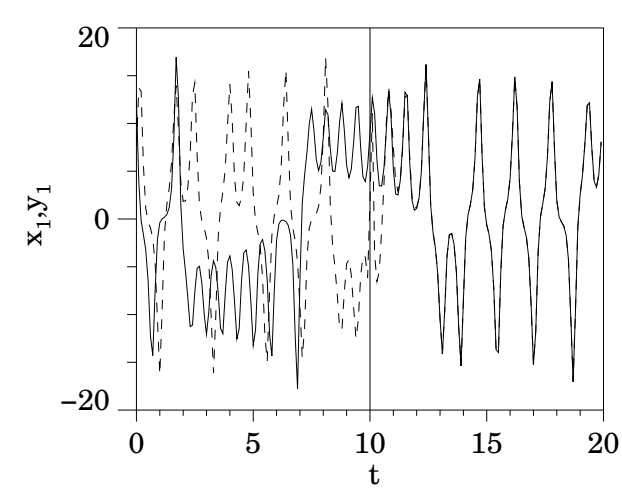

(a) $x_{1}$ und $y_{1}$ (gestrichelt) vs $t$

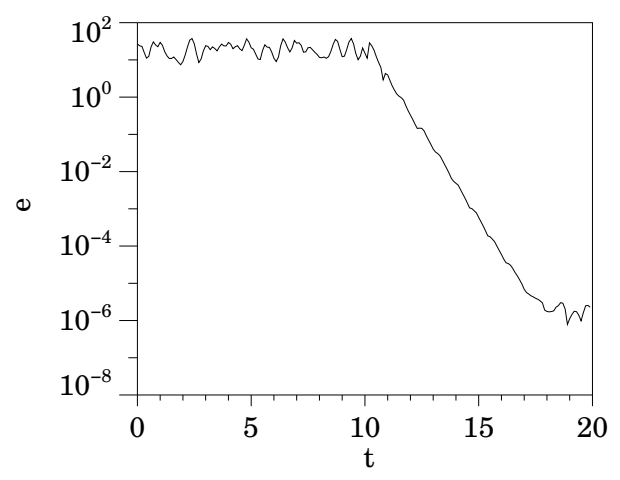

(b) $\|\mathbf{x}-\mathbf{y}\|$ vs t

Abbildung 3.20.: Synchronisation zweier unidirektional gekoppelter Lorenzoszillatoren mittels dynamischer Kopplung, welche ab dem Zeitpunkt $t=10.0$ wirksam ist (mit $\gamma=1.0$ ).

In Abb. 3.20 ist die Synchronisation ohne zusätzliches Kopplungsgewicht $(\gamma=1)$ gezeigt, wobei in Abb. 3.20a die $x_{1}$-Variable (durchgezogene Linie) mit der korrespondierenden Koordinate $y_{1}$ (gestrichelte Linie) vom getriebenen System gegen die Zeit aufgetragen ist. Zum Zeitpunkt $t=10$ wurde die Kopplung aktiviert und die Systeme synchronisieren praktisch sofort. In Abb. 3.20b ist der Synchronisationsfehler $e=\|\mathbf{x}-\mathbf{y}\|$ gegen die Zeit $t$ aufgetragen. Nach dem Einschalten der Kopplung sinkt $e$ monoton und exponentiell schnell bis hinunter auf die Größenordnung des immer hinzuaddierten Rauschens von $\sim 10^{-6}$. Offensichtlich wird die mittlere Expansion des Flusses überkompensiert, so daß die obige Überlegung die minimale Kopplungsstärke für IS überschätzt. Gleichung (3.51) ist damit eine hinreichende aber nicht notwendige Bedingung zum Erreichen von identischer Synchronisation. Falls man aber asymptotische Stabilität in jedem Punkt von $\mathcal{M}_{\text {synch }}$ fordert oder benötigt, so ist Glg. (3.51) notwendig und hinreichend.

Den Grad der transversalen (In)Stabilität kann nun durch lokale und globale Lyapunovexponenten quantifiziert werden. Der transversale Lyapunovexponent wird durch simultanes Lösen des linearisierten Gleichungssystes

$$
\dot{\mathbf{x}}_{\perp}=\underline{\underline{J}} \dot{\mathbf{y}}=\left.\underline{\underline{J}} \mathbf{f}(\mathbf{y})\right|_{\mathbf{y}=\mathbf{x}} \mathbf{x}_{\perp}+\gamma\left\langle\mathbf{v}_{\mathbf{y}} \mid \mathbf{x}_{\perp}\right\rangle \varepsilon \mathbf{v}_{\mathbf{y}}
$$

mit Glg. (3.52) bestimmt. Falls man die Tangentialvektoren $\mathbf{x}_{\perp}$ nach $\Delta t_{r}$ Zeitschritte renormiert, erhält man die lokalen und globalen transversalen Lyapunovexponenten 


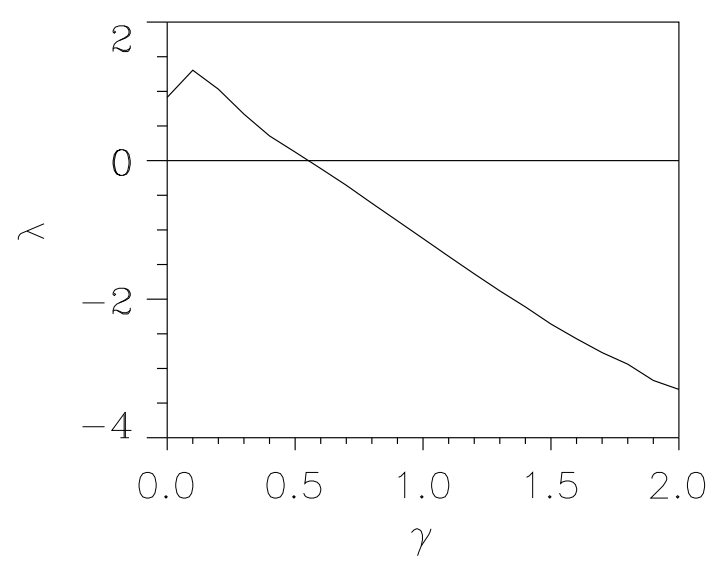

(a) $\lambda_{\perp}$ vs $\gamma$

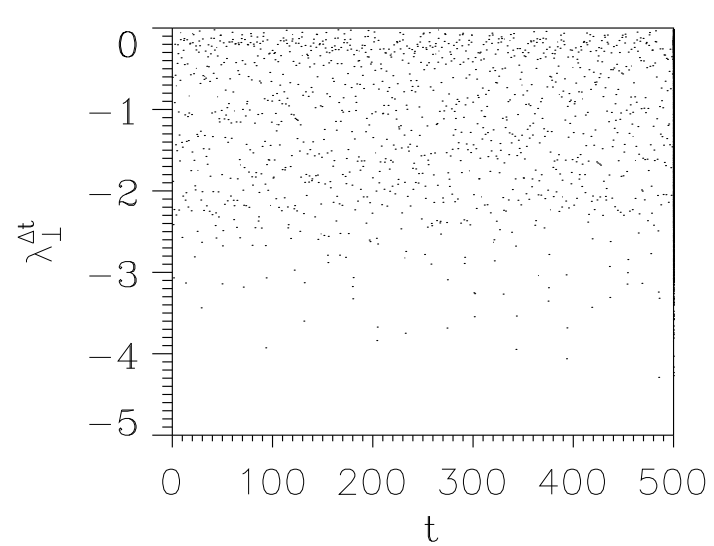

(b) $\lambda_{\perp}^{\Delta t_{r}}(t)$ vs $t$ für $\gamma=1.0$

Abbildung 3.21.: Synchronisation zweier unidirektional gekoppelter Lorenzoszillatoren mittels dynamischer Kopplung. In a) ist der transversale Lyapunovexponent $\lambda_{\perp}$ gegen das Kopplungsgewicht $\gamma$ aufgetragen, während in b) der lokale transversale Lyapunovexponent $\lambda_{\perp}^{\Delta t_{r}}(t)$ gegen die Zeit für $\gamma=1$ gezeigt ist.

durch

$$
\begin{aligned}
\lambda_{\perp}^{\Delta t_{r}}(t) & =\frac{\ln \left(\left\|\mathbf{x}_{\perp}(t)\right\|\right)}{\Delta t_{r}} \\
\lambda_{\perp} & =\lim _{n \rightarrow \infty} \frac{1}{n} \sum_{j=1}^{n} \lambda_{\perp}^{\Delta t_{r}}\left(n \Delta t_{r}\right) .
\end{aligned}
$$

Falls $\lambda_{\perp}<0$ so ist der synchrone Zustand im Mittel transversal stabil und falls $\forall t$ $\lambda_{\perp}^{\Delta t_{r}}(t)<0$ gilt, so ist $\mathcal{M}_{\text {synch }}$ global transversal stabil (innerhalb der Genauigkeit von $\Delta t_{r}$ und bezüglich des natürlichen Maßes). In Abb. 3.21a ist der transversale Lyapunovexponent $\lambda_{\perp}$ gegen das Kopplungsgewicht $\gamma$ aufgetragen. Für $\gamma \gtrsim 0.55$ ist $\lambda_{\perp}$ negativ und es wird auch stabile Synchronisation beobachtet, wobei eine längere Transiente abgewartet werden muß. Der lokale transversale Lyapunovexponent $\lambda_{\perp}^{\Delta t_{r}}(t)$ ist für $\gamma=1.0$ in Abb. 3.21b gegen die Zeit aufgetragen und ist überall kleiner oder gleich Null. $\mathcal{M}_{\text {synch }}$ ist deswegen in jedem Punkt stabil und in fast allen Punkten attraktiv, so daß transversale Instabilitäten ausgeschlossen werden können und „high-quality“-Synchronisation [33] garantiert ist. Für kleinere Kopplungsgewichte $0.55 \gtrsim \gamma<1$ ist $\mathcal{M}_{\text {synch }}$ nur im Mittel stabil, so daß positive lokale Lyapunovexponenten $\lambda_{\perp}^{\widetilde{\Delta} t_{r}}(t)$ existieren, deren Expansion aber im Mittel ausgeglichen werden kann. In Abb. 3.21a wird stabile IS auch in diesem Bereich $0.55 \gtrsim \gamma<1$ beobachtet, obwohl in diesem Fall transversale Instabilitäten (siehe 


\section{Die treibende Kraft}

auch Abschn. 2.1.1) nicht ausgeschlossen werden können. Weiterhin kann die Transientzeit zum synchronen Zustand für $\gamma$ deutlich kleiner als Eins sehr lang werden.

\subsubsection{Dynamische Kopplung bei höherdimensionalen Systemen}

Nachdem die Anwendbarkeit und Eigenschaften der dynamischen Kopplung an einem zeitdiskreten (Hénon) und zeitkontinuierlichen (Lorenz) System ausführlich diskutiert wurde, soll nun das Kopplungsschema (3.24) auf Systeme mit mehreren lokalen instabilen Richtungen verallgemeinert werden. OBdA können wir uns auf den Fall unidirektional gekoppelter Systeme beschränken, welcher für die dynamische Kopplung, wie oben gezeigt, äquivalent zur bidirektionalen Kopplung ist ${ }^{11}$. Angenommen wir haben ein dynamisches System in einem $n$-dimensionalen Phasenraum vorliegen, wobei der lokale Fluß $\Phi(\mathbf{x})$ auf dem Attraktor in maximal $M<n$ Richtungen $\mathbf{v}^{m}, m=0, \ldots, M-1$ expandierend wirkt. Die einfachste Kopplungsstrategie ist nun den Anteil von $\mathbf{x}$ in jeder dieser Richtungen zu bestimmen und als Kopplungssignal zu verwenden. Damit erhält man

$$
\mathbf{s}_{\mathbf{x}}=\left(\left\langle\mathbf{v}_{\mathbf{x}}^{0} \mid \mathbf{x}\right\rangle, \ldots,\left\langle\mathbf{v}_{\mathbf{x}}^{M-1} \mid \mathbf{x}\right\rangle\right)^{t r} \quad \text { und } \quad \mathbf{s}_{\mathbf{y}}=\left(\left\langle\mathbf{v}_{\mathbf{y}}^{0} \mid \mathbf{x}\right\rangle, \ldots,\left\langle\mathbf{v}_{\mathbf{y}}^{M-1} \mid \mathbf{x}\right\rangle\right)^{t r} .
$$

als vektorwertige Kopplungssignale, wobei die $\mathbf{v}^{0}, \ldots, \mathbf{v}^{M-1}$ die $M$ singulären Richtungen der Jacobimatrix von $\mathbf{f}$ mit Singulärwerten $w^{0}, \ldots, w^{M-1}>1$ sind. Eine kompaktere Schreibweise erhält man, wenn man von den Matrizen der Singulärwertzerlegung $\underline{\underline{\mathbf{D} \Phi}} \underline{\underline{\mathbf{V}}}=\underline{\underline{\mathbf{U}}} \underline{\underline{\mathbf{W}}}$ nur die Submatrizen, gebildet aus den ersten $M$-Spalten, verwendet. Mit den $(n \times M)$ Matrizen $\underline{\underline{\mathbf{U}}}^{M} \doteq\left(\mathbf{u}^{0}, \mathbf{u}^{1}, \ldots, \mathbf{u}^{M-1}\right), \quad \underline{\underline{\mathbf{V}}}^{M} \doteq\left(\mathbf{v}^{0}, \mathbf{v}^{1}, \ldots, \mathbf{v}^{M-1}\right)$ und $\operatorname{der}(M \times M)$ Diagonalmatrix $\underline{\underline{\mathbf{W}}}^{M} \doteq \operatorname{diag}\left\{w^{0}, \ldots, w^{M-1}\right\}$ erhält man

$$
\mathbf{s}_{\mathbf{x}}=\underline{\underline{\mathbf{V}_{\mathbf{x}}^{M}}} \mathbf{x} \text { und } \mathbf{s}_{\mathbf{y}}=\underline{\underline{\mathbf{V}_{\mathbf{y}}^{M} \mathbf{y}}}
$$

als Kopplungsvektoren. Die vollständige Kompensation der lokalen Expansion von $\mathbf{x}_{\perp}=$ $\mathbf{x}-\mathbf{y}$ kann nun durch die Kopplungsfunktion

$$
\mathbf{C}^{M}=\underline{\underline{\mathbf{U}}}^{M} \underline{\underline{\mathbf{W}}}^{M}\left(\mathbf{s}_{\mathbf{x}}-\mathbf{s}_{\mathbf{y}}\right)=\sum_{m=0}^{M-1} w^{m}\left(\mathbf{s}_{\mathbf{x}}^{m}-\mathbf{s}_{\mathbf{y}}^{m}\right) \mathbf{u}_{\mathbf{y}}^{m}
$$

erreicht werden und es ergibt sich für iterierte Abbildungen

$$
\begin{aligned}
\mathbf{x}^{n+1} & =\mathbf{f}\left(\mathbf{x}^{n}\right) \\
\mathbf{y}^{n+1} & =\mathbf{f}\left(\mathbf{y}^{n}\right)+\gamma \underline{\underline{\mathbf{U}}}
\end{aligned}
$$

als synchronisierendes Systempaar. Die zusätzliche Kopplungsstärke $\gamma$ ist, analog zum Fall einer expandierenden Richtung, größer oder gleich Eins zu wählen, um identische

\footnotetext{
${ }^{11}$ Falls bidirektionale Kopplung erforderlich sein sollte, wird die Kopplungsfunktion des getriebenen Systems durch Vertauschen von $\mathbf{x}$ und $\mathbf{y}$ in das ursprünglich treibende System eingesetzt. Zusätzlich werden die Kopplungsstärken $\gamma$ beider Systeme halbiert oder, falls ein System dominanter sein soll, entsprechend $\gamma_{\mathbf{x}}=1-\gamma_{\mathbf{y}}$ gesetzt.
} 


\section{Die treibende Kraft}

Synchronisation garantieren zu können.

Bei ODEs ist nach Abschn. 3.2.5 eine Hauptachsentransformation von der Matrix $\underline{\underline{\mathbf{B}}}=(\underline{\underline{J}} \mathbf{f})^{t r}+\underline{\underline{J} \mathbf{f}}=\underline{\mathbf{V}}^{t r} \underline{\underline{\Lambda}} \underline{\mathbf{V}}$ durchzuführen. Die Singulärwerte $w^{m}$ aus der Diagonalmatrix $\underline{\mathbf{W}}^{M}$ in Glg. (3.56) werden dann durch $\mu^{m} / 2$, wobei $\mu^{0}, \ldots, \mu^{M-1}>0$ die Eigenwerte von $\underline{\underline{\underline{\mathbf{B}}}}$ sind, ersetzt. Zusätzlich sind bei ODEs die singulären Richtungen $\underline{\underline{\mathbf{U}}} \equiv \underline{\underline{\mathbf{V}}}$ identisch und gleich der Eigenbasis von $\underline{\underline{\mathbf{B}}}$ (siehe Anhang A). Damit ergibt sich für hyperchaotische ODEs das Kopplungsschema

$$
\begin{aligned}
\dot{\mathbf{x}} & =\mathbf{f}(\mathbf{x}) \\
\dot{\mathbf{y}} & =\mathbf{f}(\mathbf{y})+\frac{\gamma}{2} \underline{\underline{\mathbf{V}}} \underline{\underline{\Lambda_{\mathbf{y}}^{M}}}\left(\mathbf{s}_{\mathbf{x}}-\mathbf{s}_{\mathbf{y}}\right),
\end{aligned}
$$

mit den Kopplungssignalen $\mathbf{s}_{\mathbf{x}}, \mathbf{s}_{\mathbf{y}}$ aus Glg. (3.55).

Da die expandierenden Richtungen entlang des Flusses im Allgemeinen nicht konstant bleiben und sich stetig verändern, kann man berechtigte Hoffnung haben, daß Synchronisation auch mit weniger als $M$ Kopplungssignalen möglich sein wird, welches dann aber nicht mehr garantiert werden kann. Falls man wieder die dynamische Kopplung im partitioniertem Phasenraum anwendet, wird man die Anzahl $M$ der Kopplungssignale an die Eigenschaften des lokalen Flusses innerhalb dieser Partition anpassen, und i.A. nur diejenigen Richtungen $\mathbf{v}^{m}$ zur Kopplung verwenden, welche Singulärwerte deutlich größer als Eins (bzw. bei ODEs Eigenwerten von $\underline{\underline{\mathbf{B}}}$ größer als Null) besitzen.

In der chaotischen Synchronisation möchte man mit möglichst wenigen (am liebsten skalaren) Kopplungssignalen auskommen. Dies sollte prinzipiell erreichbar sein, da sich aus praktisch jeder skalaren Meßgröße der vollständige Zustand nach Takens [128] rekonstruieren läßt, welches in [126] für die chaotische Synchronisation diskutiert wurde. Bei mehreren expandierenden Richtungen liegt das Problem darin, die Richtung im Phasenraum zu finden, welche zumindest teilweise der Expansion in allen instabilen Richtungen $\mathbf{v}^{0}, \ldots, \mathbf{v}^{M-1}$ gleichzeitig am Stärksten entgegenwirkt und dann die Projektion von $\mathbf{x}_{\perp}$ auf diesen Vektor zur Kopplung zu verwenden. Ein guter Ansatz ist, die sich aus dem mit den Singulärwerten gewichteten Mittelwert ergebende Richtung

$$
\overline{\mathbf{v}}=\frac{\sum_{m=0}^{M-1} w^{m} \mathbf{v}^{m}}{\sqrt{\sum_{m=0}^{M-1}\left(w^{m}\right)^{2}}},
$$

zu benutzen, wodurch den am stärksten expandierenden Richtungen des Flusses auch am meisten Rechnung getragen wird, welches in Abb. 3.22 veranschaulicht wird. Betrachtet man nun die Wirkung des linearisierten Flusses $\underline{\underline{J}} \Phi$ auf $\overline{\mathbf{v}}$,

$\underline{\underline{J}} \Phi \overline{\mathbf{v}}=\frac{\sum_{m=0}^{M-1} w^{m} \underline{\underline{\underline{J}}} \Phi \mathbf{v}^{m}}{\sqrt{\sum_{m=0}^{M-1}\left(w^{m}\right)^{2}}}=\frac{\sum_{m=0}^{M-1}\left(w^{m}\right)^{2} \mathbf{u}^{m}}{\sqrt{\sum_{m=0}^{M-1}\left(w^{m}\right)^{2}}}$, so erkennt man, daß $\overline{\mathbf{v}}$ vom lokalen Fluß um den Faktor $\bar{w}=\|\underline{\underline{J}} \Phi \overline{\mathbf{v}}\| /\|\overline{\mathbf{v}}\|=\|\underline{\underline{J}} \Phi \overline{\mathbf{v}}\|=\sqrt{\frac{\sum_{m=0}^{M-1}\left(w^{m}\right)^{4}}{\sum_{m=0}^{M-1}\left(w^{m}\right)^{2}}}$, in Richtung

$$
\overline{\mathbf{u}}=\frac{\sum_{m=0}^{M-1}\left(w^{m}\right)^{2} \mathbf{u}^{m}}{\sqrt{\sum_{m=0}^{M-1}\left(w^{m}\right)^{4}}}
$$




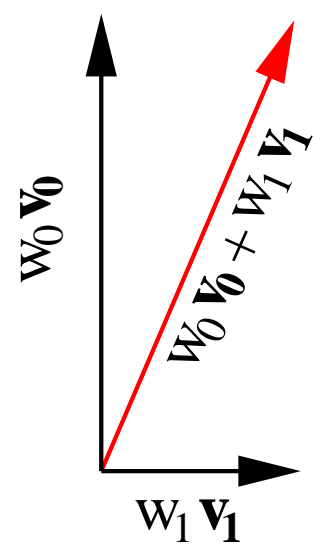

Abbildung 3.22.: Veranschaulichung der Konstruktion der mittleren instabilen Richtung bei mehreren vorhandenen expandierenden Richtungen.

expandiert wird. Mit Hilfe von $\overline{\mathbf{u}}, \overline{\mathbf{v}}$ und $\bar{w}$ erhält man damit

$$
\begin{aligned}
\mathbf{y}^{n+1} & =\mathbf{f}\left(\mathbf{y}^{n}\right)+\gamma \bar{w}\left(s_{\mathbf{x}}-s_{\mathbf{y}}\right) \overline{\mathbf{u}} \text { mit } \\
\mathbf{s}_{\mathbf{x}} & =\left\langle\overline{\mathbf{v}}_{\mathbf{x}} \mid \mathbf{x}^{n}\right\rangle \text { und } \mathbf{s}_{\mathbf{y}}=\left\langle\overline{\mathbf{v}}_{\mathbf{y}} \mid \mathbf{y}^{n}\right\rangle
\end{aligned}
$$

als Kopplungsschema für die getriebene iterierte Abbildung $\mathbf{y}^{n}$, wobei nur ein skalares Kopplungssignal benutzt wurde. Für ODEs erhält man analog

$$
\begin{aligned}
\dot{\mathbf{y}} & =\mathbf{f}(\mathbf{y})+\frac{\gamma}{2} \bar{\mu}\left(s_{\mathbf{x}}-s_{\mathbf{y}}\right) \overline{\mathbf{u}} \text { mit } \\
\mathbf{s}_{\mathbf{x}} & =\left\langle\overline{\mathbf{v}}_{\mathbf{x}} \mid \mathbf{x}\right\rangle \text { und } \mathbf{s}_{\mathbf{y}}=\left\langle\overline{\mathbf{v}}_{\mathbf{y}} \mid \mathbf{y}\right\rangle,
\end{aligned}
$$

mit

$$
\begin{aligned}
\bar{\mu} & =\sqrt{\frac{\sum_{m=0}^{M-1}\left(\mu^{m}\right)^{4}}{\sum_{m=0}^{M-1}\left(\mu^{m}\right)^{2}}} \\
\overline{\mathbf{v}} & =\frac{\sum_{m=0}^{M-1}\left(\mu^{m}\right) \mathbf{v}^{m}}{\sqrt{\sum_{m=0}^{M-1}\left(\mu^{m}\right)^{2}}} \text { und } \quad \overline{\mathbf{u}}=\frac{\sum_{m=0}^{M-1}\left(\mu^{m}\right)^{2} \mathbf{v}^{m}}{\sqrt{\sum_{m=0}^{M-1}\left(\mu^{m}\right)^{4}}}
\end{aligned}
$$

als gemittelte Instabilitätsrichtungen und Dissipationsstärke der Kopplung. Man beachte, daß aufgrund der Verwendung der gemittelten Expansionsrichtung $\overline{\mathbf{v}}$ nun trotz der Eigenwertzerlegung wieder zwei Richtungen benötigt werden.

Bei nur wenigen expandierenden Richtungen sollte man mit diesem Ansatz gute Chancen haben, Synchronisation mit den oft gewünschten skalaren Kopplungssignalen zu erreichen. Man beachte, daß sich auch unter diesen stark restriktiven Nebenbedingungen, durch den im Phasenraum lokalen Kopplungsansatz, eine dem dynamischen System angepaßte Kopplungsfunktion systematisch konstruieren läßt. 


\section{Die treibende Kraft}

\subsubsection{Informationstheoretische Aspekte}

In diesem Abschnitt soll die dymnaische Kopplung mit anderen Kopplungsschemata im Hinblick auf minimale Übertragung von Kopplungsinformationen des Systems x verglichen werden. Für die dynamische Kopplung scheint „sporadic driving“ am wenigsten Information über das getriebene System zu benötigen, um Synchronisation zu erreichen. Dazu muß beim Hénonsystem nur alle $\Delta t=6$-Iterationen gekoppelt werden. Im Folgenden wird mit Hilfe der Informationstheorie eine obere Grenze für den Abstand $\Delta t$ zwischen zwei Kopplungseingriffen abgeleitet. Aufgrund der sensitiven Abhängigkeit von den Anfangsbedingungen, läßt sich aus einem zum Zeitpunkt $t_{0}$ mit Unsicherheit $\varepsilon$ gemessenen Zustand $\mathbf{x}$ die weitere Zeitentwicklung für $t>t_{0}$ nur mit (exponentiell) wachsender Unsicherheit vorhersagen. Aus diesem Grund lassen sich chaotische dynamische Systeme als Informationquellen auffassen, d.h. falls man nicht regelmässig den Zustand durch Messung observiert, kann man nach einiger Zeit keine vernünftigen Vorhersagen über den Ort des aktuellen Zustand mehr machen, obwohl der Zustand zur Zeit $t_{0}$ sehr genau bekannt war. Uns interessiert hier, nach welcher Zeit $\Delta t$ jegliche Information des gemessenen Zustandes aus dem aktuellen verschwunden ist. Bei dieser Abtastrate $\Delta t$ ist jegliche Redundanz aus der Zeitreihe verschwunden, so daß Zustände welche für $t=t_{0}$ in einer Box mit Radius $\varepsilon$ lagen, nach dieser Zeit den ganzen Attraktor $\mathcal{A}$ bedecken. $\Delta t$ ist damit die maximale Zeit in der Vorhersagen über die Zeitentwicklung des Systemes überhaupt nach brauchbare Informationen über zukünftige Zustände liefern können. Im Kontext der Synchronisation bedeutet dies, daß nach dieser Zeit zwei mit Abstand $\varepsilon$ benachbarte Trajektorien vollständig desynchronisiert worden sind, so daß $\Delta t$ auch die obere Grenze für den Abstand zwischen zwei Messungen und Kopplungseingriffe definiert, welche überhaupt zur Synchronisation führen können. Dies würde einer Kopplung entsprechen, welche alle $\Delta t$ Schritte den kompletten Zustand $\mathbf{y}$ wieder in die aktuelle Box des treibenden Systems bringt, so daß das getriebene System danach den Zustand des treibenden vorhersagt. Im weiteren soll nun $\Delta t$ abgeschätzt werden, wozu wir einige Begriffe aus der Informationstheorie dynamischer Systeme benötigen ${ }^{12}$. Im Folgenden gehen wir davon aus, daß ein partitionierter Phasenraum (3.45) mit $M$ Boxen der Größe $\varepsilon$ vorliegt. Dadurch liegt ein diskretes Alphabet von Symbolen (Boxen) vor und eine Messung liefert die Nummer der Box, in welcher sich der aktuelle Zustand gerade befindet. Durch eine solche Messung gewinnen wir im Mittel über das System die Information

$$
\overline{\mathrm{I}}(\varepsilon)=-K \sum_{k=1}^{M(\varepsilon)} p_{k} \operatorname{ld}\left(p_{k}\right),
$$

wobei $p_{k}$ die Aufenthaltswahrscheinlichkeit in der Box $B_{k}$ darstellt und ld der Logarithmus zur Basis 2 ist, so daß die Information in bits gemessen wird. Die freie Konstante $K$ wird im weiteren zu Eins gesetzt. Die Information welche man über den Zustand des Systemes erhält, hängt von der Genauigkeit $\varepsilon$ der Messung (Feinheit der Partitionierung) ab, so daß wie zu erwarten eine bessere Messung auch mehr Information liefert. Betrachtet man eine Reihe von $n$ aufeinanderfolgenden Messungen, so läßt sich der Grad der

\footnotetext{
${ }^{12}$ Für eine detailliertere Diskussion der Informationstheorie dynamischer Systeme sei z.B. auf [5] verwiesen.
} 


\section{Die treibende Kraft}

Unsicherheit zukünftiger Zustände aus den mit der Unsicherheit $\varepsilon$ gemessenen Zustände durch die Kolmogorov-Sinai (KS) Entropie charakterisieren. Die KS-Entropie stellt die obere Grenze der mittleren Informationsproduktion des Systems pro Zeiteinheit dar und wird in $\frac{b i t s}{s e c}$ gemessen. Diese Größe steht im engem Zusammenhang mit den positiven Lyapunovspektrum, welches die sensitive Abhängigkeit von den Anfangsbedingungen quantifiziert und damit letztendlich auch einen Indikator für Informationsproduktion darstellt. Die KS-Entropie kann unter gewissen Vorraussetzungen durch die Summe der positiven Lyapunovexponenten

$$
\mathrm{h}_{\mu}=\sum_{i}^{+} \lambda_{i}
$$

berechnet werden ${ }^{13}$, wobei das Lyapunovspektrum hier mit dem Logarithmus zur Basis 2 berechnet wird. Die maximale Vorhersagezeit $\Delta t$ eines gemessenen Zustandes kann man als den Zeitabstand abschätzen, an dem das System wieder soviel neue Information produziert hat wie man aus der Messung erhalten hat. Die Gleichgewichtsbedingung $\overline{\mathrm{I}}(\varepsilon)-\Delta t \mathrm{~h}_{\mu}=0$ bestimmt den Zeitabstand

$$
\Delta t(\varepsilon)=\frac{\overline{\mathrm{I}}(\varepsilon)}{\mathrm{h}_{\mu}},
$$

nachdem jegliche Information aus einer Messung mit Genauigkeit $\varepsilon$ wieder verloren ist. Dieser Zeitpunkt hängt von der Genauigkeit der Messung $\varepsilon$ ab, welche in diesem Fall der Feinheit der Partitionierung entspricht, so daß eine genauere Messung, wie auch zu erwarten, prinzipiell eine längere Vorhersage ermöglicht. Für die Synchronisation bedeutet dies, daß Observablen, welche mit einer Abtastrate größer $\Delta t$ aufgenommen wurden, prinzipiell nicht zur Synchronisation führen können, da unabhängig von der Kopplung die Systeme nach $\Delta t$ desynchronisiert sind. $\Delta t, \mathrm{~h}_{\mu}, \overline{\mathrm{I}}(\varepsilon)$ sind Größen, welche durch Mittelung über das natürliche Maß entstehen, so daß sie auch nur statistisch das Verhalten des Systems beschreiben. Lokal werden diese Größen auf dem Attraktor stark schwanken und damit manche Trajektorienabschnitte länger vorhersagbar sein als andere. Die Synchronisation soll aber global stabil sein und die gekoppelten Systeme dürfen sich auch nur einen Bruchteil von der Attraktorausdehnung voneinander entfernen, so daß praktisch der maximal erlaubte zeitliche Kopplungsabstand deutlich unter dem idealen Wert aus Glg. (3.66) liegen wird. Für die Hénonabbildung lassen sich die KS-Entropie und Information mit den Partitionierungen aus Abschn. 3.2.4 und dem größten Lyapunovexponenten leicht numerisch abschätzen. Die folgende Tabelle zeigt den mit Glg. (3.66) berechneten und den numerisch beobachteten maximalen Meßabstand, ab der Zustände aus einer Box den ganzen Attraktor bedecken, in Abhängigkeit der Meßgenauigkeit (Feinheit der Partitionierung) $\varepsilon$.

\footnotetext{
${ }^{13}$ Für genauere Informationen zu den Vorraussetzungen für dieses Resultat wird hier auf weiterführende Literatur verwiesen. Im Allgemeinen wird die KS-Entropie durch die Summe $\sum_{i}^{+} \lambda_{i}$ nach oben abgeschätzt.
} 
3. Die treibende Kraft

\begin{tabular}{|c|c|c|c|}
\hline$\varepsilon$ & $\Delta t_{\text {theoretisch }}(\varepsilon)$ & $\Delta t_{\text {numerisch }}(\varepsilon)$ & $\Delta t_{\text {synch }}(\varepsilon)$ \\
0.1 & 10 & 6 & \\
0.01 & 17 & 9 & \\
$10^{-3}$ & 24 & 14 & 1 \\
$10^{-5}$ & 37 & 22 & 9 \\
$10^{-8}$ & 56 & 35 & 22 \\
$10^{-10}$ & 70 & 45 & 33 \\
\hline
\end{tabular}

Unsere simple Abschätzung überschätzt das numerisch beobachtete $\Delta t$ um $\approx 33 \%$ und spiegelt daher die Verhältnisse ganz gut wider. Die rechte Spalte zeigt den numerisch bestimmten maximalen zeitlichen Abstand $\Delta t_{\text {synch }}(\varepsilon)$ unter der Nebenbedingung, daß alle $\Delta t_{\text {synch }}(\varepsilon)$ der Zustand vollständig ersetzt wird und der Synchronisationsfehler $e=\|\mathbf{x}-\mathbf{y}\|<10^{-3}$ bleibt, welches oben als Schwellwert zur stabilen Synchronisation der Hénonsysteme benutzt wurden ist. $\Delta t_{\text {synch }}(\varepsilon)$ beschreibt die Zeit nach der innerhalb der Meßgenauigkeit $\varepsilon$ identisch synchronisierte Systeme den Abstand $e=\|\mathbf{x}-\mathbf{y}\|=10^{-3}$ überschreiten und ist hier die entscheidende Größe. Im Abschnitt über die Synchronisation zweier dynamisch gekoppelter Hénonabbildungen hatten wir durch das additive Rauschen eine Meßgenauigkeit von $10^{-8}$. Mit der besten Kopplungsstrategie, der periodischen Ersetzung des kompletten Zustandes $\mathbf{y}^{n}=\mathbf{x}^{n}+\eta^{n}, \eta^{n} \leq 10^{-8}$, muß alle 22-Iterationen gekoppelt werden, um Synchronisation während der restlichen Zeitentwicklung sicherzustellen. Der Nachteil ist, daß der komplette Zustand übertragen werden muß. Falls man nur die $y_{1}^{n}$-Komponente durch $x_{1}^{n}$ ersetzt, muß man mindestens alle 4Iterationen koppeln, um Synchronisation garantieren zu können. Bei der in der Literatur sehr häufig benutzten Kopplungsstrategie, der Ersetzung von $\left(y_{1}^{n}\right)^{2}$ im getriebenen System durch $s=\left(x_{1}^{n}\right)^{2}$, ist sogar Kopplung in jeder Iteration notwendig. Die dynamische Kopplung muß mindestens alle 6-Iterationen angewandt werden (siehe Abschn. 3.2.3), damit bei $\varepsilon=10^{-8}$ die Systeme sich nicht um mehr als $e=\|\mathbf{x}-\mathbf{y}\|=10^{-3}$ voneinander entfernen. Dies ist zwar nur ca. $30 \%$ des optimalen Wertes von $\Delta t_{\text {synch }}(\varepsilon)=22$, aber man darf nicht vergessen, daß die lokale Kopplung nur ein skalares Kopplungssignal benötigt, weswegen sie dann auch im Hinblick auf minimale Informationsübertragung schon fast $60 \%$ des idealen Wertes erreicht.

\subsubsection{Diskussion und Ausblick}

Durch die dynamische Kopplung kann jedem Punkt auf dem Attraktor eine dynamisch optimale und eindeutige Kopplungsfunktion zugeordnet werden, wobei die Meßfunktion nur die zur Synchronisation essentielle Informationen bereitstellt. Aus dem Blickpunkt der nichtlinearen Kontrolltheorie, siehe Abschn. 3.1.5, entspricht dies einem lokalen Beobachter. Dieser Beobachter hat aber keine feste Blickrichtung auf das System, sondern observiert genau an den Stellen, an denen neue Information produziert wird, welche er zur Rekonstruktion des Gesamtzustandes benötigt. Man könnte deswegen die dynamische Kopplung auch als dynamischen Beobachter bezeichnen, welcher nur dahin schaut wo auch etwas neues passiert. Aus diesem Grund ist die dynamische Kopplung sicher ein interessanter Ansatz im Hinblick auf die systematische Konstruktion von nichtlinearen Beobachtern auch in zeitkontinuierlichen Systemen. 


\section{Die treibende Kraft}

Ein Schwachpunkt der dynamischen Kopplung in der vorgestellten Form ist sicherlich, daß in die Meß- und Kopplungsfunktion der vollständige Zustandsvektor eingeht, und damit auch einer Messung zugänglich bzw. das System in allen Komponenten beeinflußbar sein muß. Diese Forderung wird bei realen Systemen wohl nur selten zu erfüllen sein, ändert aber nichts am Interesse an einer dynamisch motivierten systematischen Konstruktion einer Kopplung für beliebige Systeme. Allerdings kann man durch Betrachtung dieser idealen Situation die Anteile des Zustandes bzw. der Systemgleichungen feststellen, welche für die Beobachtbarkeit und Steuerbarkeit des Systemes wichtig oder eher unwichtig sind. Falls nun nicht der komplette Zustand einer Messung zugänglich ist, kann man durch Betrachtung der Richtung der Kopplungsfunktion $\sim \mathbf{u}^{i}$ entscheiden, ob und wo das System im Phasenraum mit den vorhandenen Größen überhaupt zu synchronisieren ist, bzw. mit welchen Meßgrößen wenig oder kein Einfluß auf die Synchronisation genommen werden kann. Falls eine Meßgröße $s=\mathbf{h}(\mathbf{x})$ einen wesentlichen Anteil an den expandierenden Richtungen in allen Partitionen besitzt, kann man guter Hoffnung sein, daß mittels dieses Signals alleine auch Synchronisation erreichbar ist, so daß sich durch die Analyse der lokalen Stabilitätseigenschaften zumindest eine suboptimale Kopplung innerhalb der gegebenen Möglichkeiten konstruieren lassen sollte. Bei der Hénonabbildung identifiziert man aus Abb. 3.14 sofort die $x_{1}$-Komponente als entscheidende Meßgröße an den Rändern des Hénonattraktors, wo die Instabilität auch nach Abb. 3.4c am stärksten ist, während um $x_{1}=0$ die $x_{2}$-Komponente die meiste Information über die lokale Instabilität liefert. Der linke Teil von Abb. 3.14 zeigt, daß durch Beeinflussung des Systems in der $x_{2}$-Komponente praktisch keine Synchronisation erzielt werden kann.

Die OGY-Methode [80] aus der nichtlinearen Kontrolltheorie geht von praktisch der gleichen Idee, der Ausnutzung der lokalen Stabilitätseigenschaften des Flusses $\Phi$ zur Erreichung der Zieldynamik, aus, weswegen im weiteren die Unterschiede diskutiert werden sollen. Bei der OGY-Kontrollmethode können instabile chaotische Orbits, welche im chaotischen Attraktor eingebettet sind, stabilisiert werden. Dazu wird i.A. aus einer Poincaré-Abbildung die linearisierte Dynamik in der Nähe dieses Orbits durch die Jacobi-Matrix approximiert und der Einfluß der Variation eines zugänglichen Parameters des Systems auf die lokalen Stabilitätseigenschaften benutzt, um das System durch kleine Parameteränderungen auf die stabile Mannigfaltigkeit des Orbits zu befördern. Eine Verbesserung der Stabilisierung liefert die quasikontinuierliche OGY-Steuerung [28, 44, 109], welche mehrere Poincaré-Abbildungen benutzt um öfter steuernd eingreifen zu können, wodurch auch höherperiodische bzw. instabilere Orbits stabilisiert werden können. Der Hauptunterschied zur dynamischen Kopplung liegt darin, daß die OGY-Methode zur Stabilisierung bekannter (periodischer) Lösungen des Systems optimiert ist, während die dynamische Kopplung den aktuellen Zustand eines chaotischen Attraktors liefert, ohne durch Variation eines Parameters in die Dynamik einzugreifen. Die Zielsetzung der Synchronisation ist es, einen unbekannten Zustand zu beobachten und nicht einen bekannten zu kontrollieren. Nur zu diesem Zweck wird ein getriebenes System konstruiert welches mit dem treibenden, vertreten durch das Meßsignal mittels der Kopplungsfunktion synchronisiert. Die OGY-Methode macht auch keine Aussagen welche Parameter zur Steuerung zu verwenden sind bzw. überhaupt geeignet sind, während die dynamische 


\section{Die treibende Kraft}

Kopplung konstruiert wurde,

- um auch eine Meßfunktion zu liefern, welche maximale Information über die lokale Instabilität der Synchronisation enthält und

- eine Kopplungsfunktion zu definieren, welche diese Information optimal ausnutzt.

Beide Methoden haben nur gemein, daß sie die lokale Instabilität kompensieren und die lokale Anziehung zur Erreichung der Zieldynamik ausnutzen, sie verfolgen aber durchaus unterschiedliche Zielsetzungen und können daher nur bedingt direkt miteinander verglichen werden.

Bei zukünftigen Untersuchungen zur dynamischen Kopplung könnte z.B. versucht werden bessere Strategien, als etwa das „sporadic driving“ aus Abschn. 3.2.3, zu finden, um noch weniger Kopplungsinformation zur Synchronisation übertragen zu müssen. Auch das Problem des Auffindens der relevanten Meß- und Steuergrößen aus den lokalen Stabilitätseigenschaften dürfte gerade auch bei technischen Anwendungen einiges Potential für intensivere Untersuchungen besitzen. Im Bereich der sicheren Nachrichtenübertragung mittels Synchronisation besitzt die dynamische Kopplung auch einiges Potential, weil durch sie die Redundanz im Übertragungssignal drastisch vermindert werden kann (siehe Abschn. 3.2.7). Eine typische Attacke auf chaotischer Synchronisation basierende "Kryptosysteme“ ist die Rekonstruktion der Systemdynamik aus den Übertragungssignalen, welches bei verminderter Redundanz des Signales stark erschwert wird. Beim Hénonsystem mußte z.B. nur jede 6'te Iteration gekoppelt werden, so daß eine solche Attacke die Dynamik der 6-fach iterierten Hénonabbildung rekonstruieren muß, welche eine weitaus komplexere Struktur als die einfache besitzt. Weiterhin ist der Zustand $\mathbf{x}$, in welchem i.A. das Informationssignal moduliert wird, in den Überragungssignalen schon durch $s_{x}=\langle\mathbf{v} \mid \mathbf{x}\rangle$ mit den aktuellen expandierenden Richtungen, welche vom Zustand $\mathbf{x}$ abhängen, kodiert, welches den Angriff zusätzlich erschweren sollte. Eine genauere Untersuchung eines auf der dynamischen Kopplung basierenden chaotischen Kryptosystems ist von uns noch nicht durchgeführt wurden. Das Hauptproblem liegt in dem Nachweis der kryptographischen Sicherheit, wo allgemein für auf chaotischer Synchronisation beruhender Systeme noch kaum Ergebnisse existieren. Ohne jegliche bewiesene Aussagen über die Sicherheit eines Kryptosystems, macht es aber i.A. keinen Sinn, ein solches ernsthaft vorzuschlagen, weswegen wir bis jetzt diese Anwendung nicht weiter verfolgt haben. 


\section{Synchronisation und Kontrolle räumlich ausgedehnter Systeme}

Die nichtlineare Dynamik hat in den letzten zwei Dekaden eine Vielzahl von Phänomenen und Eigenschaften dynamischer System entdecken und erklären können, welche in linearen Systemen prinzipiell nicht auftreten. Zum Studium dieser Effekte wurden hauptsächlich niederdimensionale chaotische Systeme, wie iterierte Abbildungen und ODEs herangezogen, welche praktisch alle komplexen Phänomenen zeigen, aber noch von der Struktur und Dynamik einfach genug sind, um sie oft sogar analytisch behandeln zu können. Inzwischen kann die Dynamik von niederdimensionalen chaotischen Systemen als gut verstanden angesehen werden. Ähnliches gilt für die nichtlineare Zeitreihenanalyse, so können Zeitreihen aus niederdimensionalen Systemen gut von stochastischen Signalen unterschieden („surrogate data“), der Attraktor rekonstruiert, das dynamische System lokal und global modelliert und die Zeitentwicklung (für nicht zu lange Zeiten) vorhergesagt werden. Reale dynamische Systeme sind oft aber weitaus komplexer, man denke z.B. an das Gehirn oder das Wetter um ein paar Extreme zu nennen, so daß manchmal der Vorwurf gemacht wird, daß sich die nichtlineare Dynamik hauptsächlich mit Spielzeugsystemen („,toy systems“) beschäftigt und die Methoden bei hochdimensionalen realen Systemen nicht anwendbar sind. Solchen Vorwürfen könnte man entgegnen: Zum jetzigen Zeitpunkt noch nicht, aber in naher Zukunft. In der Tat fokussiert sich das Interesse der aktuellen Forschung (nicht nur in der nichtlinearen Dynamik) immer mehr auf hochdimensionale Systeme. Ob nun grundlegende Prozesse der Strukturbildung oder die Dynamik von vielen interagierenden Systemen untersucht werden, so kann man nun auf ein fundiertes Grundgerüst aus dem niederdimensionalen Chaos zurückgreifen, welches sich für das Verständnis dieser weitaus komplexeren Systeme mit vielen neuartigen Phänomenen als sehr wertvoll herausstellt. In diesem Kapitel werden wir uns nur partiell mit der Eigendynamik räumlich ausgedehnter Systeme beschäftigen, welche zwar für sich genommen schon hochinteressant ist und i.a. noch viele Ansatzpunkte zur Forschung besitzen wird, aber weit über das Thema und Volumen dieser Arbeit hinausgehen würde. Raumzeitliches Chaos begegnet einem auch oft bei wichtigen technischen Anwendungen, z.B. bei Turbulenz [1], Plasmainstabilitäten [101], multimodalen Lasern [138] und Reaktions-Diffusions-Gleichungen [103], wobei dann Bedarf an möglichen Kontrollstrategien besteht. In der Biologie werden häufig gekoppelte niederdimensionale Systeme zur Modellierung von erregbaren Medien, wie z.B. kardiale, neuronale und retinale Gewebeschichten, verwendet [71]. Wir beschränken uns in dieser Arbeit auf die Synchronisation von zwei gekoppelten identischen räumlich ausgedehnten Systemen, 
welche raumzeitliches Chaos zeigen, d.h. die Dynamik ist nicht nur in der Zeit sondern auch entlang der jetzt vorhandenen räumlichen Ausdehnung irregulär. Im Hinblick auf die gerade genannten Anwendungen, scheint die chaotische Synchronisation von solchen Systemen erst einmal nur von akademischen Interesse zu sein, aber die zur Synchronisation verwendeten Kopplungsstrategien lassen sich natürlich auch für Kontrollzwecke verwenden, so daß sich die gewonnenen Erkentnisse auch leicht auf solche Anwendungen übertragen lassen. Andererseits dürfte die Synchronisation raumzeitlicher Systeme zur Zustandsrekonstruktion auch Anwendungen bei raumzeitlichen Datensätzen besitzen. Abschnitt 4.1 diskutiert wie Synchronisation bei Feldern von gekoppelten niederdimensionalen Systemen erreicht werden kann. In Abschn. 4.2 wird eine Kopplungsmethode vorgestellt, welche auch bei Systemen mit kontinuierlichen Raumkoordinaten, beschrieben durch partielle Differentialgleichungen, angewendet und neben der Synchronisation auch zur Unterdrückung bzw. Kontrolle von hochdimensionalem Chaos verwendet werden kann. Das partielle Synchronisation, wie Phasen- und Frequenzsynchronisation, auch bei raumzeitlichen Systemen beobachtet werden kann, wird in Abschn. 4.4 demonstriert.

\subsection{Felder gekoppelter niederdimensionale Systeme}

Das einfachste Modell von raumzeitlichem Chaos besteht darin, viele einzelne selbsterregte niederdimensionale (chaotische) Oszillatoren miteinander interagieren zu lassen, womit sich z.B. die kollektive Dynamik gekoppelter Neuronenverbände modellieren läst. Die Dynamik des Gesamtsystems hängt entscheidend von der Kopplung der Oszillatoren untereinander ab. Falls man alle Elemente global durch ihren momentanen Mittelwert („mean field“) koppelt, erhält man für 1-dimensionale diskrete Oszillatoren

$$
u_{i}^{n+1}=(1-\varepsilon) f\left(u_{i}^{n}\right)+\frac{\varepsilon}{N} \sum_{j=1}^{N} f\left(u_{j}^{n}\right) .
$$

In [121] wurden die Oszillatoren durch die logistische Abbildung modelliert, wobei der Bifurkationsparameter zufällig um den Mittelwert $a$ variert wurde. Für $\varepsilon=0$ liegen $N$ unabhängige chaotische Abbildungen vor, welche makroskopisch wie mikroskopisch hochdimensionales Chaos zeigen. Bei $\varepsilon>0$ wirkt das gemittelte Kopplungsfeld

$$
h=\frac{\varepsilon}{N} \sum_{j=1}^{N} f\left(u_{j}^{n}\right)
$$

regularisierend auf die Dynamik, so daß die makroskopische Dynamik $h$ des Gesamtsystems niedrigdimensional erscheint. Falls die Einzeloszillatoren aber nicht miteinander (verallgemeinert) synchronisiert sind, (typischerweise im Bereich $0<\varepsilon \ll 1$ ), so wird die wahre (mikroskopische) Dynamik von $\mathbf{u}=\left(u_{i}^{1}, \ldots, u_{i}^{N}\right)^{t r}$ weiterhin hochdimensional bleiben, so daß die Komplexität des Systems abhängig von der Beobachtungsskala ist [121].

Ein weitere wichtige Modellklasse sind Felder von diffusiv gekoppelten (chaotischen) 


\section{Synchronisation und Kontrolle räumlich ausgedehnter Systeme}

iterierten Abbildungen

$$
\begin{aligned}
u_{i}^{n+1} & =F\left(u_{i-1}^{n}, u_{i}^{n}, u_{i+1}^{n}\right) \\
& =(1-\delta) f\left(u_{i}^{n}\right)+\frac{\delta}{2}\left[f\left(u_{i-1}^{n}\right)+f\left(u_{i+1}^{n}\right)\right],
\end{aligned}
$$

welche im folgenden als CML ${ }^{1}$ abgekürzt werden. Die Kopplung entspricht dem diskretisierten Laplaceoperator $\Delta$, wobei die nächsten Nachbarelemente mit der Diffusionskonstante $\delta$ gekoppelt sind. Im hier betrachteten 1-dimensionalen Fall erhält man bei festen Randbedingungen $u_{1}^{n}=u_{N}^{n}=$ konst. eine Kette und bei periodischen Randbedingungen $u_{1}^{n}=u_{N}^{n}$ einen Ring von gekoppelten Oszillatoren. Diese Systeme zeigen eine reichhaltige und hochgradig komplexe Dynamik, welche bis heute noch nicht vollständig verstanden und Gegenstand aktueller Forschung ist, siehe z.B. [102] für einen Überblick und weiterführende Referenzen.

Im weiteren beschränken wir uns auf zwei gekoppelte identische 1-dimensionale CMLs der Form (4.2), welche miteinander (identisch) synchronisiert werden sollen. Die am häufigsten benutzte Kopplungstechnik zur Synchronisation und auch zur Kontrolle diffusiv gekoppelten CMLs ist die sogenannte „future“-Kopplung

$$
\begin{aligned}
v_{i}^{n+1} & =F\left(v_{i-1}^{n}, v_{i}^{n}, v_{i+1}^{n}\right)-\varepsilon\left[F\left(v_{i-1}^{n}, v_{i}^{n}, v_{i+1}^{n}\right)-F\left(u_{i-1}^{n}, u_{i}^{n}, u_{i+1}^{n}\right)\right] \\
& =v_{i}^{\prime n+1}-\varepsilon\left(v_{i}^{\prime n+1}-u_{i}^{n+1}\right),
\end{aligned}
$$

wobei bei jedem Einzeloszillator $v_{i}$, der Zustand $u_{i}$ des korrespondierenden Oszillators im treibenden System, diffusiv durch den Term $\varepsilon\left[F\left(v_{i-1}^{n}, v_{i}^{n}, v_{i+1}^{n}\right)-F\left(u_{i-1}^{n}, u_{i}^{n}, u_{i+1}^{n}\right)\right]$, zurückgekoppelt wird. Da $F$ die Dynamik der Einzeloszillatoren beschreibt, werden die zukünftigen Zuständen, welche sich für das ungekoppelte System ergeben würden, diffusiv zurückgekoppelt, was den Namen „future“-Kopplung rechtfertigt. Das Prinzip ist graphisch in Abb. 4.1a illustriert. Ein Grund warum man nicht den aktuellen Zustand $u_{i}^{n}$ zurückkoppelt liegt darin, daß der Attraktor iterierter Abbildungen oft kein globales Bassin besitzt und außerhalb des Bassins die Trajektorien meist divergieren (siehe z.B. das Bassin des Hénon-Attraktors in Abb. 3.2). Weiterhin besteht die Zeitentwicklung von iterierten Abbildungen aus (großen) „Sprüngen“ im Phasenraum, so daß ein Kopplungsterm der Form $\varepsilon\left(v_{i}^{n}-u_{i}^{n}\right)$ eine Korrektur des Zustandes zur Zeit $n$ bewirkt, während sich das System schon durch $v_{i}^{\prime n+1}=F\left(v_{i-1}^{n}, v_{i}^{n}, v_{i+1}^{n}\right)$ in einem möglicherweise weit entfernten Teil des Phasenraumes befindet und die Kopplung dann sogar destabilisierend wirken, oder sogar zum Verlassen das Bassins führen, kann. In CMLs sind benachbarte Oszillatoren durch den Diffusionsterm der Stärke $\delta$ untereinander schon stark gekoppelt, weswegen diese oft auch nicht voneinander unabhängig und damit korreliert sind. Aus diesem Grund ist es wenig überraschend, daß nicht alle Elemente des CMLs paarweise gekoppelt werden müssen und eine periodische Anordnung von Kontrollelementen mit Abstand $d$ oft ausreichend zur Synchronisation bzw. Kontrolle ist. Dies führt auf die „pinning"-Kopplung [42], welche in Abb. 4.1b gezeigt ist. Die zweite Zeile von Glg. (4.3) zeigt, daß die „future“-Kopplung äquivalent zur partiellen Zustandsersetzung (bei diskreter Dynamik) aus Abschnitt 3.1.4 ist. Dabei werden zunächst das treibende und das

\footnotetext{
${ }^{1}$,coupled map lattices“
} 
(ungekoppelte) getriebene System iteriert und danach der Zustand des getriebenen System korrigiert. Es zeigt sich, daß oft auch nicht zu jeder Iteration, sondern nur alle $T$ Iterationen gekoppelt werden muß [62]. Dies führt auf ein raumzeitliches „sporadic

a)

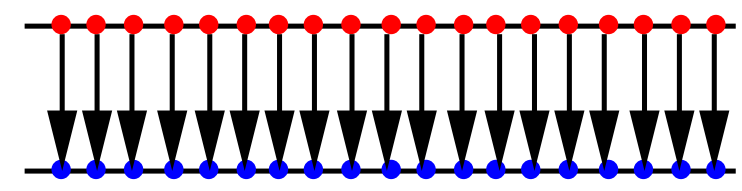

b)

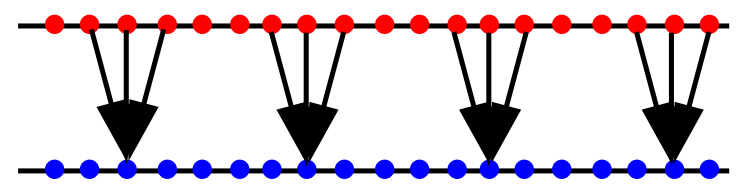

c)

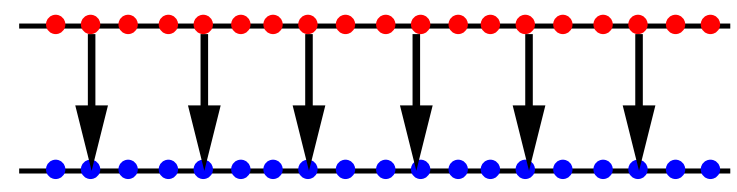

d)

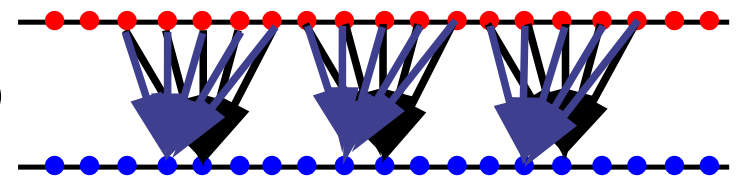

Abbildung 4.1.: Prinzip der wichtigsten Kopplungsvarianten von Feldern gekoppelter iterierter Abbildungen bzw. ODEs. a) global, b) diskret im Raum [42], c) partielles Ersetzen [65] und d) optimerte „pinning“ Kopplung [38]

driving“"

$$
\begin{aligned}
v_{i}^{n} & \rightarrow v_{i}^{n}-\varepsilon^{\prime}\left(v_{i}^{n}-u_{i}^{n}\right) \quad \varepsilon^{\prime}= \begin{cases}\varepsilon & \text { falls } n \bmod T \text { und } i \bmod d \\
0 & \text { sonst }\end{cases} \\
v_{i}^{n+1} & =F\left(v_{i-1}^{n}, v_{i}^{n}, v_{i+1}^{n}\right),
\end{aligned}
$$

welches in Abb. 4.1c veranschaulicht ist. Der Unterschied zur „future“-Kopplung liegt darin, daß nun vor der Iteration die partielle Ersetzung vorgenommen wird, was mathematisch äquivalent ist, aber die zusätzliche Übertragung der nächsten Nachbarn von $u_{i}$ zur Kopplung einspart. Die ungekoppelten Bereiche des getriebenen Systems erhalten jetzt nur noch indirekt Informationen aus dem treibenden System, welche von den „pinning“-Punkten aus, durch den internen Diffusionsmechanismus des CML, transportiert wird. Im Allgemeinen kann deswegen der räumliche Abstand $d$ der Kontrollelemente und 
auch der zeitliche Abstand $T$ zwischen zwei Kopplungsereignissen bei chaotischer Dynamik nicht zu groß gewählt werden und liegt oft bei wenigen Elementen bzw. Iterationen. Abbildung 4.1d zeigt das Prinzip der optimierten „pinning“-Kopplung nach [37, 38]. Es wird eine mit Hilfe der nichtlinearen Kontrolltheorie optimierte Kopplung berechnet, welche dann in zwei „pinning“-Punkten die gemessenen Signale durch Rückkopplung einbringt. Bei diesem Ansatz erhalten Elemente des treibenden CML, welche weit von den Kopplungspunkten entfernt liegen, größeres Gewicht in der Kopplungsfunktion als benachbarte Elemente, so daß die interne Diffusion auch besser Information über weit entfernte Elemente in die ungekoppelten Bereiche transportieren kann. Mit dieser Strategie wird die Anzahl der Kontrollelemente im Vergleich zur „pinning“-Kopplung stark reduziert.

Alle vorgestellten Kopplungstechniken wurden in den letzten Jahren erfolgreich zur Synchronisation und Kontrolle bzw. Unterdrückung von raumzeitlichem Chaos bei CMLs angewendet [37, 42, 50, 65, 67, 85, 139] und können auch für Systeme, deren Einzelelemente zeitlich kontinuierliche Dynamik zeigen, angewendet werden. So wurde die Synchronisation von diffusiv gekoppelten Feldern von gewöhnlichen Differentialgleichungen $\left(\mathrm{CODE}^{2} \mathrm{~s}\right)$ u.a. in $[62,65]$ demonstriert.

\subsection{Partielle Differentialgleichungen}

In diesem Kapitel soll der Hauptaugenmerk auf Systeme mit kontinuierlichen Raumund Zeitvariablen, natürlicherweise beschrieben durch partielle Differentialgleichungen $\left(\mathrm{PDE}^{3} \mathrm{~s}\right)$, gerichtet werden. Wir beschränken uns oBdA auf ein Paar unidirektional gekoppelter identischer 1-dimensionaler partieller Differentialgleichungen der Form

$$
\frac{\partial u}{\partial t}=F\left(u, \frac{\partial u}{\partial x}, \frac{\partial^{2} u}{\partial x^{2}}, \ldots\right), \quad x \in[0, L]
$$

mit räumlicher Ausdehnung L. Man spricht von identischer Synchronisation von zwei gekoppelten dynamischen Systemen falls die Zeitentwicklung der Zustände nach einer gewissen Einschwingzeit übereinstimmt, d.h. $\|u(t)-v(t)\| \rightarrow 0$ für $t \rightarrow \infty$. Für räumlich ausgedehnte Systeme sind nun die Zustände $u(x, t)$ und $v(x, t)$ selbst kontinuierliche Funktionen der Raumkoordinate und der Zustandsraum ist unendlichdimensional. In diesem Fall präzisieren wir die obige Definition zu:

Definition 4.1 (Synchronisation von räumlich ausgedehnten dyn. Systemen) Zwei räumlich ausgedehnte dynamische Systeme heißen identisch synchronisiert, falls der Abstand ihrer Zustände innerhalb der gesamten räumlichen Ausdehnung $0 \leq x \leq L$ gegen Null konvergiert, d.h. für 1-dimensionale PDEs muß gelten

$\forall x \in[0, L]: \lim _{t \rightarrow \infty}\|u(x, t)-v(x, t)\|=0$.

Für $x \in \mathbb{Z}$ und $t \in \mathbb{Z}$ ist das die Definition für identische Synchronisation von CMLs, für $x \in \mathbb{Z}$ und $t \in \mathbb{R}$ für CODEs und für $x, t \in \mathbb{R}$ für PDEs. Analog zum Fall von gekoppelten

\footnotetext{
${ }^{2}$, coupled ordinary differential equation"

3 "partial differential equation"
} 


\section{Synchronisation und Kontrolle räumlich ausgedehnter Systeme}

niedrigdimensionalen Oszillatoren, existiert wieder eine invariante Unterannigfaltigkeit $u(x) \equiv v(x)$, die Synchronisationsmannigfaltigkeit, deren Stabilitätseigenschaften das Auftreten von stabiler „high quality" Synchronisation [33] bestimmen. Die Mannigfaltigkeit ist asymptotisch stabil und („high quality“) Synchronisation tritt auf, falls das transversale System $w_{\perp}(x, t)=\frac{1}{2}[u(x, t)-v(x, t)]$ einen asymptotisch stabilen Fixpunkt im Ursprung besitzt. In der Tat lassen sich alle bekannten Nachweisverfahren von niederdimensionalen Systemen [18, 32, 93, 96, 99] verallgemeinern und auch auf diese Systemklasse anwenden. Dazu zählen notwendige Kriterien, wie bedingte Lyapunovexponenten $\lambda_{C}$, aber auch hinreichende Kriterien, wie die Konstruktion von Lyapunovfunktionen und die Stabilität invarianter Untermengen, wie z.B. aller in den Attraktor eingebetteten UPOs. Aus diesem Grund erwartet man auch ähnliche Schwierigkeiten, wie z.B. das Auftreten von On-Off Intermittenz aus Abschnitt 2.1.1, welche weiter unten auch beobachtet werden. Es stellt sich nun die Frage ob und wie sich die oben vorgestellten Kopplungstechniken bei diskreter Raumausdehnung $u_{i}, i \in \mathbb{Z}$ (CMLs, CODEs) auf Systeme mit kontinuierlichen Raum $u(x), x \in \mathbb{R}$ (PDEs) verallgemeinern lassen. In [127] wurde vorgeschlagen die Kopplung im gesamten Raum wirken zu lassen, so daß man bei der dort verwendeten dissipativen Kopplung

$$
\frac{\partial v}{\partial t}=F\left(v, \frac{\partial v}{\partial x}\right)+\varepsilon(u-v)
$$

als getriebenes System erhält. Bei mehrdimensionaler räumlicher Ausdehnung ist auch eine komplette Ersetzung einer räumlich ausgedehnten Komponente des Zustandsvektors á la Pecora\&Carroll denkbar. Diese Varianten sind z.B. in der Biologie bei Membransystemen oder Reaktions-Diffusionssystemen relevant, wenn räumlich ausgedehnte Konzentrationen der Reaktanden auf mehrere Systeme einwirken können und dadurch eine Kopplung zwischen den Systemen realisiert wird [71]. Für die Kontrolle, Identifikation und Synchronisation räumlich ausgedehnter Systeme möchte man dagegen ein gegebenes System unter Benutzung von möglichst wenig Information und Kontrolleingriffen auf die Zieldynamik führen, wodurch es wichtig wird nicht entlang der gesamten räumlichen Ausdehnung $x$ messen bzw. koppeln zu müssen. In einigen numerischen Arbeiten [11, 64] wurde deswegen die „pinning“-Kopplung auf den sich aus der Diskretisierung der PDE ergebenden CML angewendet, wodurch chaotische Synchronisation zweier unidirektionale gekoppelter PDEs im gesamten Raum erreicht werden konnte. Ein wesentliches Problem bei der Anwendung von diesen auf diskreten Raum optimierten Kopplungstechniken auf PDEs ist, daß die Kopplung und Messung nun in infinitesimal kleinen Punkten erfolgt, welches vom physikalischen und praktischen Standpunkt aus in realen Systemen nicht möglich ist. Die numerische Lösung einer PDEs wird zwar häufig durch raumzeitliche Diskretisierung auf die Iteration eines CML reduziert, welches aber nur eine Approximation darstellt. Weiterhin muß die Diskretisierung viel feiner gewählt werden als alle interessierenden dynamischen Strukturen, so daß numerische Artefakte aufgrund der Diskretisierung in den Ergebnissen ausgeschlossen werden können. Die oben vorgestellten Kopplungstechniken für CMLs greifen aber direkt bei den Einzelabbildungen, also auf der Skala der Diskretisierung, an, so daß sie bei kontinierlichen Raum nicht anwendbar sind. Die Arbeiten [11, 64] sollten deswegen nur als erster Schritt zur 
Synchronisation und Kontrolle von PDEs aufgefasst werden, weil aufgrund der benutzten „pinning“-Kopplung die Ergebnisse von der Art und der Feinheit der benutzten Diskretisierung abhängen werden.

\subsection{Synchronisation mit Sensoren}

Aus diesen Gründen haben wir in [54, 56, 57, 95, 90, 130] die Sensor-Kopplung vorgeschlagen, welche unter Beibehaltung der Effektivität der diskreten Kopplungstechniken auch bei kontinuierlichem Raum numerisch wie experimentell anwendbar ist. Die Idee ist, daß typische experimentelle Meßsensoren und Aktuatoren eine begrenzte Auflösung $l$ besitzen, weswegen sie immer auf einer räumlich begrenzten Region der Ausdehnung $l$ wirksam sind. Eine Meßvorschrift der Form

$$
\bar{u}_{n}(t)=\frac{1}{l} \int_{n d-l / 2}^{n d+l / 2} u(t) d x, \quad n=1, \ldots, N
$$

wird nach [57, 130] im folgenden als Sensor bezeichnet, welcher einen räumlichen Mittelwert der lokalen Dynamik, in einen Intervall der Länge $l$ an der Stelle $x$, von einer räumlich ausgedehnten Observable $u$ liefert. Man kann bei einem Sensor z.B. an das Ausgangssignal einer Photodiode oder Videokamera denken ${ }^{4}$. Das rechte Bild von Abb. 4.2 zeigt eine typische raumzeitliche chaotische Lösung einer PDE (hier Kuramoto-Sivashinski), wobei mittels dreier Sensoren skalare Zeitreihen gemessen wurden, welche zur Visualisierung über die Originaldynamik geplottet wurden. Durch die begrenzte räumliche Auflösung erhält man nun gemittelte Information von lokalen Teilen des Systems und kann nicht mehr alle feinen Details der Systemdynamik auflösen, wodurch auch Information über den exakten Zustand verlorenzugehen scheint. Im folgenden demonstrieren wir aber, daß mittels dieser verwaschenen Sensorzeitreihen der vollständige Systemzustand durch Synchronisation wieder rekonstruiert werden kann und das räumlich ausgedehnte Meß- und Kontrollapparate sogar noch von Vorteil dabei sind. Zur Synchronisation betrachten wir zwei identische PDEs der Form (4.5) mit räumlicher Länge $L$ und plazieren im treibenden System $N$ Sensoren der Ausdehnung $l$ mit konstantem Abstand $d=\frac{L}{N}$, wodurch wir $N$ skalare Zeitreihen $\bar{u}_{1}, \ldots, \bar{u}_{N}$ als Kopplungssignale erhalten. Nun müssen wir noch eine Kopplungstechnik aus Abschn. 3 auswählen, welche räumlich lokal die Kopplungssignale ins getriebene System zurückkoppelt. Wir verwenden im gesamten Kapitel die dissipative Kopplung, wobei die Sensorsignale $\bar{u}_{n}$ aber auch mit anderen Kopplungstechniken verwendet werden können. Zur konkreten Realisierung werden nun im getriebenen System an den gleichen Stellen $N$ Sensorsignale $\bar{v}_{1}, \ldots, \bar{v}_{N}$

\footnotetext{
${ }^{4}$ Reale Meßsensoren werden nicht immer so scharfe Intervallgrenzen besitzen, sondern oft nur einen gewichteten Mittelwert liefern, wobei die Gewichtungsfunktion z.B. durch eine Gaußglocke angenähert werden kann, so daß die Information im Zentrum gegenüber den Randgebieten verstärkt berücksichtigt wird. Die genaue Form der Wichtungsfunktion ist für das folgende Kopplungsschema aber nicht wichtig, der entscheidende Punkt ist die Verwendung von räumlich ausgedehnten Meß- und Kontrollgeräten.
} 

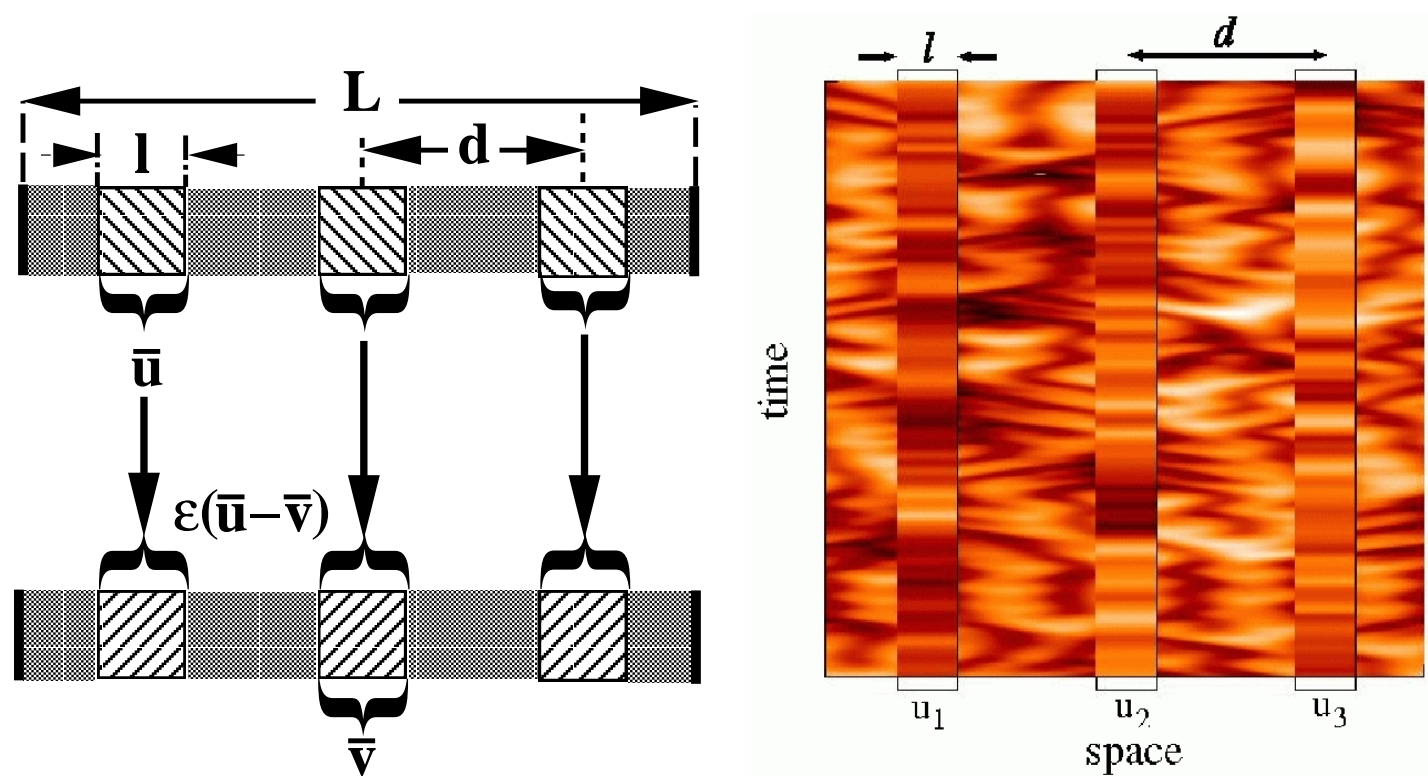

Abbildung 4.2.: Links: Prinzip der Sensorkopplung. Rechts: Visualisierung von drei gemessenen Sensorzeitreihen, welche über die zeitliche Entwicklung einer raumzeitlich chaotischen Lösung der Kuramoto-Sivashinski Gleichung geplottet wurde.

gemessen und der dissipative Kopplungsterm mit Stärke $\varepsilon$ an jeder Position $n=1, \ldots, N$

$$
\frac{\partial v}{\partial t}=F\left(u, \frac{\partial v}{\partial x}, \frac{\partial^{2} v}{\partial x^{2}}, \ldots\right)+f\left(\bar{u}_{n}, \bar{v}_{n}\right), \quad x \in[0, L]
$$

durch räumlich ausgedehnte Aktuatoren

$$
f\left(\bar{u}_{n}, \bar{v}_{n}\right)=\left\{\begin{array}{cll}
\epsilon\left(\overline{u_{n}}-\overline{v_{n}}\right) & : & n d-l / 2 \leq x \leq n d+l / 2 \\
0 & : & \text { sonst }
\end{array}\right.
$$

angewendet. Im linken Teil von Abb. 4.2 ist das Prinzip nochmals mit $N=3$ Sensoren veranschaulicht.

\subsubsection{Kuramoto-Sivashinski Gleichung}

Als erstes numerisches Beispiel betrachten wir die Kuramoto-Sivashinski (KS) Gleichung [24], welche allgemein Systeme naher einer linearen Instabilität beschreibt. Das 1-dimensionale Modell wird zur Beschreibung der diffusen Instabilität von Flammengrenzen, Flüssigkeitsgrenzschichten, die Phasendynamik von Reaktions-Diffusions-Systemen und auch Scherinstabilitäten dünner fliessender Schichten verwendet. Es existieren mehrere Varianten der KS-Gleichung, welche alle aus der 1-dimensionalen Grundgleichung hervorgehen $\frac{\partial \phi}{\partial t}+\frac{\partial^{2} \phi}{\partial x^{2}}+\frac{\partial^{4} \phi}{\partial x^{4}}+\left(\frac{\partial \phi}{\partial x}\right)^{2}=0$ hervorgehen. In der Literatur wird fast ausschließlich die Gleichung für das Geschwindigkeitsfeld $u=\frac{\partial \phi}{x}$ behandelt, welche auf die 
Standardform der KS-Gleichung $\frac{\partial u}{\partial t}+\frac{\partial^{2} u}{\partial x^{2}}+\frac{\partial^{4} u}{\partial x^{4}}+2 u \frac{\partial u}{\partial x}+\eta u=0$ führt, wobei oft ein zur Originalgleichung zusätzlicher Dämpfungsterm $\eta u$ eingeführt wird. Der Dämpfungsterm stabilisiert bei periodischen Randbedingungen die Grundmode, welche für $\eta=0$ instabil ist und zur Divergenz der Lösungen führt. In diesem Kapitel behandeln wir ausschließlich die Variante aus [24, 70]

$$
\frac{\partial u}{\partial t}=-\frac{\partial^{2} u}{\partial x^{2}}-\frac{\partial^{4} u}{\partial x^{4}}-2 u \frac{\partial u}{\partial x}
$$

im Intervall $[0, L]$ mit festen Randbedingungen $u=\frac{\partial u}{\partial x}=0$ für $x=0$ und $x=L$, wodurch auch ohne Dämpfungsterm die Stabilität der Lösungen garantiert ist. Die räumliche Länge $L$ ist in dieser Form der einzige freie Parameter der KS-Gleichung und für $L>L_{0} \approx 20$ tritt raumzeitliches Chaos auf, das in Abb. 4.3 für $L=100$ gezeigt ist. Zur Integration der PDE haben wir für alle Rechnungen ein explizites Euler-Schema zur

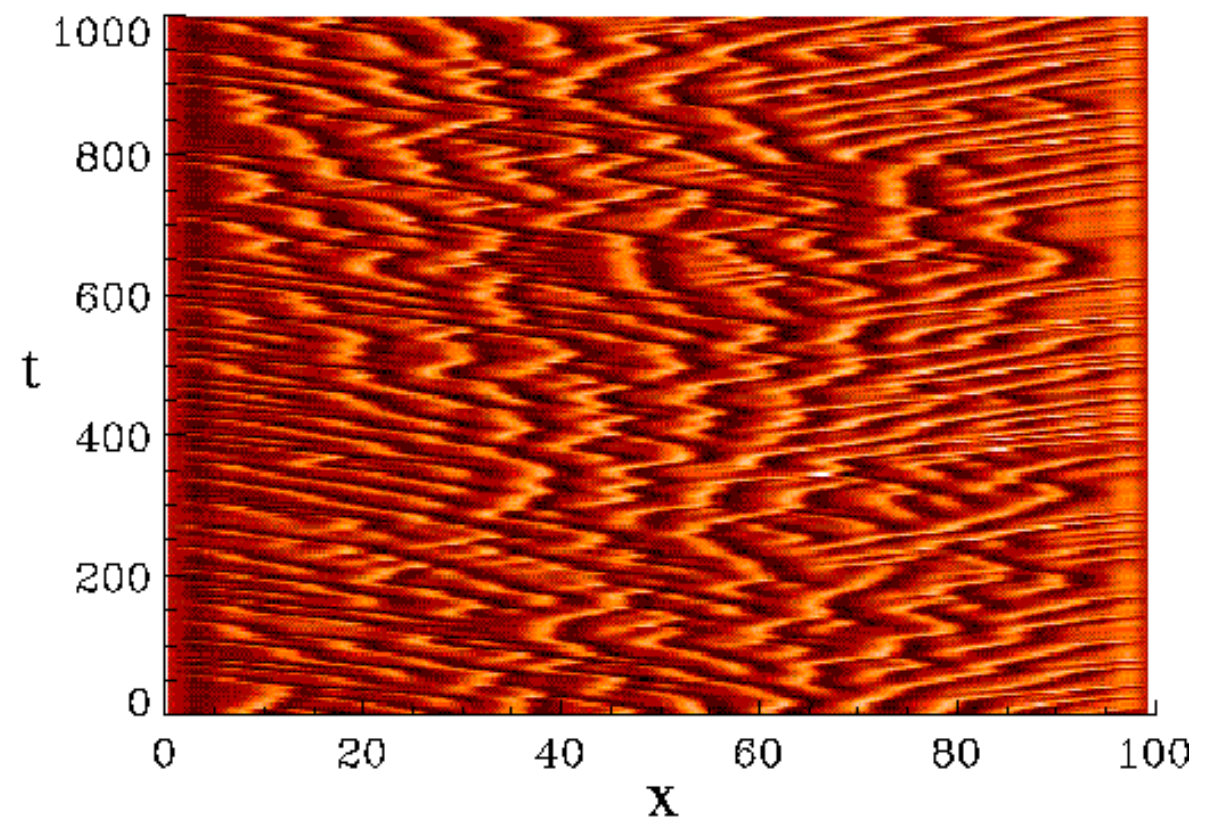

Abbildung 4.3.: Raumzeitlich chaotische Dynamik der Kuramoto-Sivashinski Gleichung bei einer räumlichen Länge von $L=100$.

Diskretisierung, mit $\Delta x=0.25$ Gitterpunkten pro Einheitslänge und einer Schrittweite von $\Delta t=0.001$, benutzt. Weiterhin zeigt die Kuramoto-Sivashinski Gleichung extensives Chaos, das heißt alle extensiven Größen, wie z.B. Entropie, Attraktordimension, etc., skalieren linear mit der Systemgröße $L$ [24]. Zur Überprüfung haben wir die Lyapunovdimension ${ }^{5} D_{L}$ in Abhängigkeit der Systemlänge $L$ numerisch berechnet. Die Ergebnisse

\footnotetext{
${ }^{5}$ Die Lyapunovdimension ist definiert durch $D_{L}=k+\frac{\sum_{i=1}^{k} \lambda_{i}}{\lambda_{k+1}}$, wobei $k$ als die Zahl definiert ist, wo $\sum_{i=1}^{k} \lambda_{i} \geq 0$ aber $\sum_{i=1}^{k+1} \lambda_{i}<0$ ist. Im Computerexperiment, wo das Lyapunovspektrum mehr oder
} 


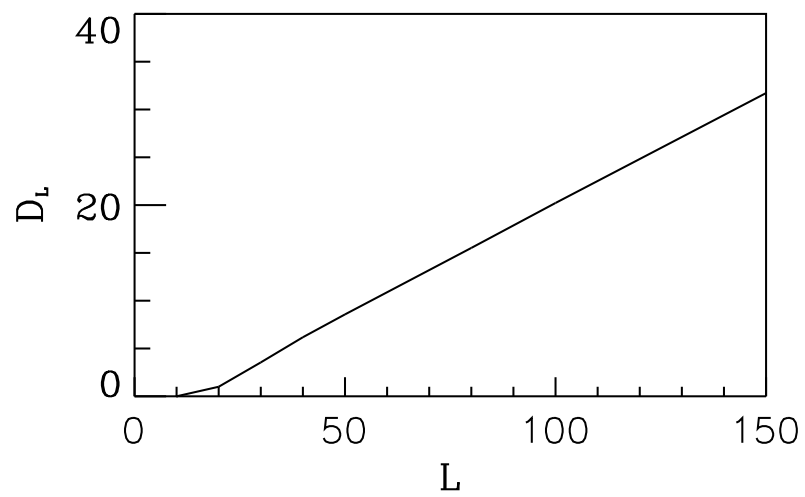

Abbildung 4.4.: Lyapunovdimension $D_{L}$ der treibenden Kuramoto-Sivashinski Gleichung (4.10) gegen die Systemlänge $L$.

sind in Abb. 4.4 gezeigt, wo eine praktisch perfekte Gerade auftritt. Lineare Regression liefert die Beziehung $D_{L}=0.232 L-2.99$ [130], welche in sehr guter Übereinstimmung mit den frühen Ergebnissen von [70] $\left(D_{L}=0.230 L-2.70\right)$ ist $^{6}$. Gleichung (4.10) treibt eine zweite KS-Gleichung

$$
\frac{\partial v}{\partial t}=-\frac{\partial^{2} v}{\partial x^{2}}-\frac{\partial^{4} v}{\partial x^{4}}-2 v \frac{\partial v}{\partial x}+f\left(\bar{u}_{n}, \bar{v}_{n}, x\right), \quad n=1, \ldots, N,
$$

wobei $f\left(\bar{u}_{n}, \bar{v}_{n}, x\right)$ aus Glg. (4.9) die Kopplung mit den $N$ gemessenen Sensorsignalen realisiert. Analog zum Fall der Synchronisation von iterierten Abbildungen mit der „pinning"-Kopplung aus Abschn.4.1, wird bei gegebener Länge $L$ eine minimale Anzahl $N$ von Kopplungssignalen zur Synchronisation von Glg. (4.11) mit Glg. (4.10) benötigt, welche von der Kopplungsstärke $\varepsilon$ und nun aber auch von der Breite $l$ der Sensoren abhängt. Dabei werden die Systeme nach obiger Definition als synchronisiert betrachtet, falls der lokale Synchronisationfehler $e(\mathbf{x}, t)=u(\mathbf{x}, t)-v(\mathbf{x}, t)$ in allen Gitterpunkten gegen Null strebt und nach einer gewissen Transientzeit keine Intermittenz beobachtet wird. Zuerst wollen wir untersuchen wie die minimale Anzahl $N$ von Sensoren, zur Erreichung des synchronen Zustandes, von der räumlichen Ausdehnung des Systems $L$ abhängt. Dazu haben wir bei fester Kopplungsstärke $\varepsilon$ und Breite $l$ der Sensoren, $N$ in Abhängigkeit der Länge $L$ bestimmt. Abbildung 4.5 zeigt, daß $N$ für mehrere Kombinationen von $l$ und $\varepsilon$ linear mit der Systemlänge $L$ skaliert und sich damit wie eine extensive Größe $N \sim L$ verhält. Mittels linearer Regression und unter Benutzung von $D_{L}=0.232 L-2.99$ aus

weniger leicht berechenbar ist, läßt sich durch $D_{L}$ ein Maß für die Attraktordimension auch von hochdimensionalen Systemen, wie bei uns PDEs, bestimmen.

${ }^{6} \mathrm{Zu}$ dieser Zeit (1985) war die Berechnung der Lyapunovdimension $D_{L}$ von PDEs selbst auf den damals größten verfügbaren Supercomputern, eine sehr rechenintensive und sehr sehr zeitintensive Aufgabe, so daß die Leistung von [70] zu dieser Zeit als sehr hoch einzuschätzen ist. 


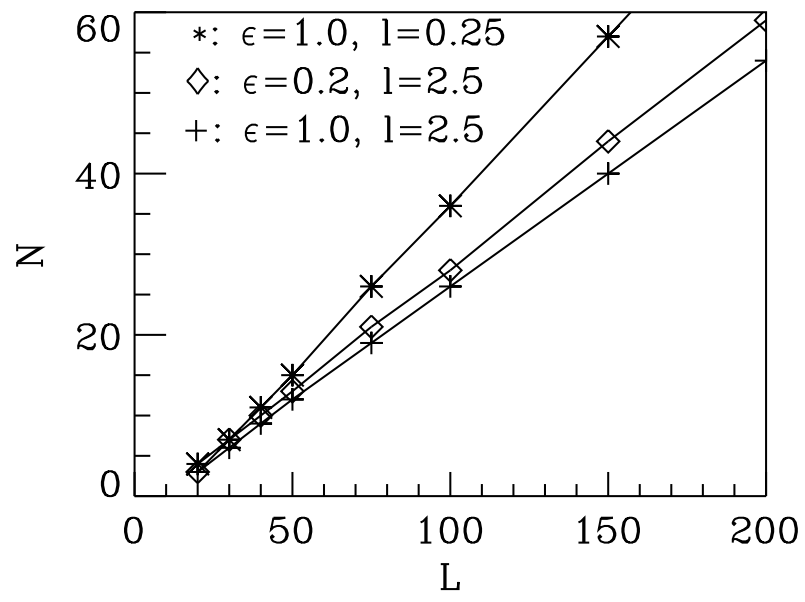

Abbildung 4.5.: Minimale Anzahl $N$ von Kopplungssignalen zur Erreichung von stabiler Synchronisation mittels der Sensorkopplung aus Glg. (4.9) in Abhängigkeit der Systemlänge $L$.

Abb. 4.4 kann man die räumliche Länge $L$ eliminieren und erhält

$$
\begin{aligned}
& N=1.81 D_{L}-0.34 \quad: \quad \varepsilon=1.0, \quad l=0.25 \\
& N=1.32 D_{L}+1.67 \quad: \quad \varepsilon=0.2, \quad l=2.5 \\
& N=1.21 D_{L}+1.61 \quad: \quad \varepsilon=1.0, \quad l=2.5 \text {. }
\end{aligned}
$$

Die minimale Anzahl der Kopplungssignale $N$ hängt damit bei gegebenen Kopplungskonstanten $l$ und $\varepsilon$ nur von der Attraktordimension $D_{L}$ bzw. von der räumlichen Ausdehnung des Systems $L$ ab. Daraus folgt direkt, daß der maximale Abstand $d$ zwischen zwei Sensoren für die KS-Gleichung nicht von der Systemlänge abhängt und ähnlich wie die Dimensionsdichte ( $D_{L}$ pro Längeneinheit) eine Invariante des Systems ist. Aus diesem Grund ist es ausreichend, die gegenseitige Abhängigkeit zwischen den drei Kopplungsparametern $\varepsilon, l$ und $N$ bei zwei festen ansonsten aber beliebigen räumlichen Längen $L$ von Glg. (4.10) und Glg. (4.11) zu untersuchen. Die erhaltenen Ergebnisse können dann aufgrund der linearen Skalierung auf beliebige Längen $L$ extrapoliert werden. Das Ergebnis, daß mehr Kontrollelemente für größere Systeme benötigt werden, ist nicht überraschend, da die ungekoppelten Bereichen zwischen den Sensoren nur indirekt, durch die der PDE eigenen räumlichen Diffusion, mit dem treibenden System gekoppelt sind und das chaotische Verhalten räumliche Korrelationen exponentiell zerfallen läßt. Aus diesem Grund darf der Abstand zwischen zwei Sensoren nicht zu groß gewählt werden, anderenfalls können die ungekoppelten Bereiche im getriebenen System eine vom treibenden unabhängige Eigendynamik entwickeln und es wird dort Desynchronisation auftreten. Die Beziehungen in Glg. (4.12) deuten an, daß die wenigsten Kopplungssignale bei großer Kopplungsstärke $\varepsilon$ und großer Breite $l$ der Sensoren benötigt werden, wobei $N$ empfindlicher von der Breite $l$, als von der Stärke der Kopplung $\varepsilon$, abhängt. Eine genauere Untersuchung ist in Abb. 4.6 dargestellt, wo für eine feste Länge $L=50$, die Beziehung zwischen $N$ und der Breite $l$ für verschiedene Kopplungsstärken $\varepsilon$ aufgetragen sind. Die 


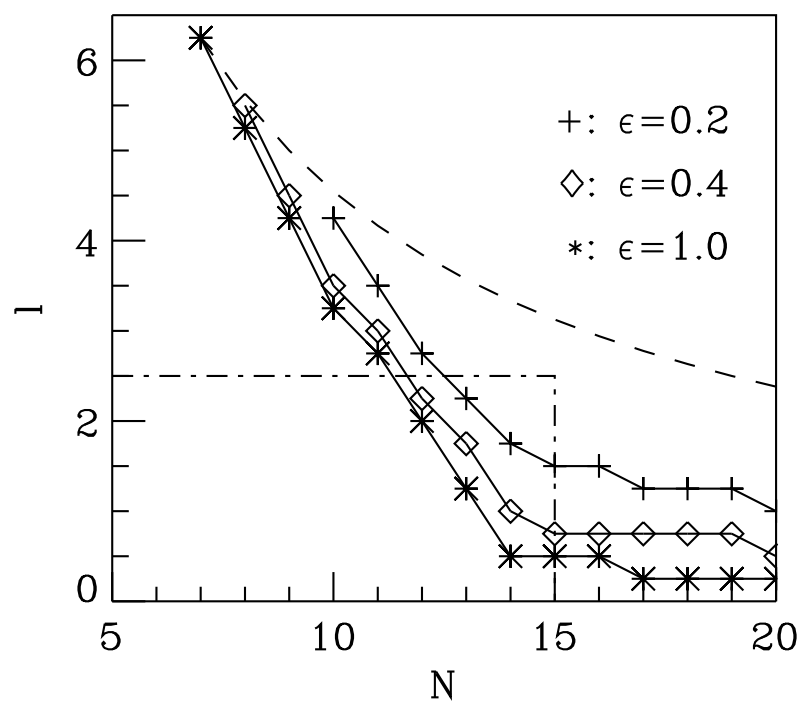

Abbildung 4.6.: Minimale Anzahl $N$ von Kopplungssignalen zur Erreichung von stabiler Synchronisation mittels der Sensorkopplung aus Glg. (4.9) in Abhängigkeit der Breite $l$ der Sensoren, bei konstanter Systemlänge von $L=50$.

gestrichelte Linie deutet die kritische räumliche Breite $l_{\max }$ an, ab der die Sensoren anfangen sich zu überlappen, welches eine obere Grenze der Anzahl der Sensoren bei gegebener Breite $l$ definiert. Man erkennt, daß je breiter man die Sensoren wählt, desto weniger Kopplungssignale $N$ werden zur Synchronisation benötigt. Es existiert aber für eine gegebene Kopplungsstärke $\varepsilon$ und Breite $l$ eine minimale Anzahl von Sensorsignalen $(\approx 6$ in diesem Beispiel). Eine Erhöhung der Kopplungsstärke alleine bewirkt nur eine kleine Verminderung in der Anzahl der benötigten Sensoren. Für $l \rightarrow 0$ erhalten wir den Grenzfall der „pinning“-Kopplung [38], wir benötigen aber jetzt viel mehr Kontrollelemente als im Fall von räumlich ausgedehnten Sensoren/Aktuatoren. Bei der benutzten Gitterauflösung von $\Delta x=0.25$ entspricht $l=0.25$ der „pinning“-Kopplung. Im Hinblick auf die Anzahl $N$ der benötigten Kopplungssignale ist damit die Sensorkopplung der „pinning“-Kopplung deutlich überlegen. In einem gewissen Sinn liegt der Preis für die Reduzierung der übertragenen Information in der nun größeren zu kontrollierenden bzw. gekoppelten Fläche. Ein weiterer Grenzfall entspricht dem Übergang von $l \rightarrow 0$ unter Beachtung von $\varepsilon \propto 1 / l$ (i.e. $\varepsilon \rightarrow \infty$ ), so daß in den Punkten $x=n d$, $n=1,2, \ldots, N$ die Variable $v$ durch $u$ ersetzt wird. Dies entspricht der Synchronisation in „pinning"-Punkten unter Benutzung der vollständiger Zustandsersetzung und wurde z.B. in [64, 65] behandelt. Den maximalen Abstand $d$ zwischen zwei Sensoren kann man auch theoretisch grob abschätzen. Dazu betrachten wir die raumzeitlichen linearen Korrelation der Zustände [24, 29] bei einer typischen Dynamik der freien KS-Gleichung, welche in Abb. 4.7 gezeigt sind. Der Referenzustand, bezüglich dessen die Korrelationen in Raum und Zeit berechnet wurden sind, ist durch die senkrechte schwarze Linie in den Einzelbildern angedeutet. Im linken Bild befinden wir uns exakt in der Mitte des 

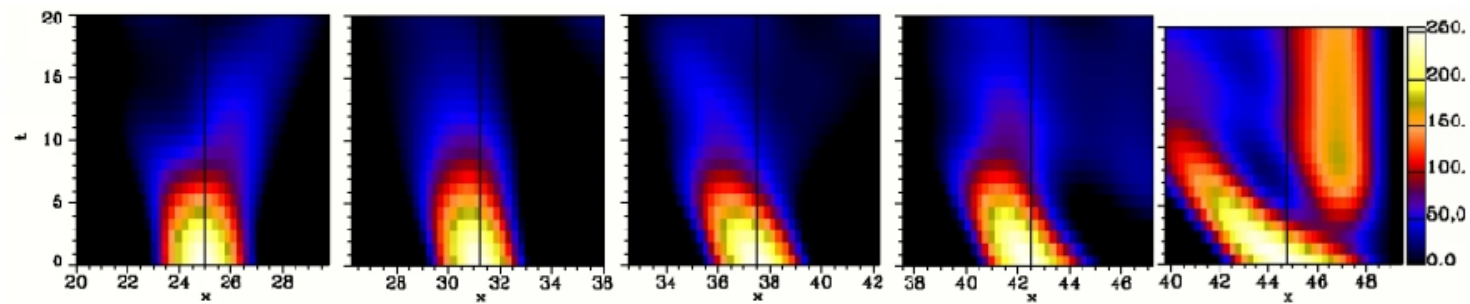

Abbildung 4.7.: Abhängigkeit der räumlichen Korrelationen innerhalb der KS-Gleichung mit $L=50$ (4.10) mit festen Randbedingungen. Der Abstand des Bezugszustandes (senkrechte schwarze Linie) zu den Rändern nimmt von links nach rechts ab. Die Korrelation nimmt von Weiß nach Schwarz ab, wobei beliebige Einheiten verwendet wurden, und ist beim Übergang von Rot nach Blau um ca. das $\frac{1}{e}$-fache abgefallen.

Raumes bei $x=25$ und man erkennt den exponentiellen Zerfall raumzeitlicher Korrelationen, wobei offensichtlich die Korrelation zeitlich langsamer abnimmt als räumlich. Die (räumliche) Korrelationslänge $\xi_{X}$ wird nun als der räumliche Abstand definiert, an dem die Korrelation auf das $\frac{1}{e}$-fache abgefallen ist. Aufgrund der festen Randbedingungen wird das Verhalten zu den Rändern hin regulärer, welches sich in der Zunahme von $\xi_{x} \approx 1.5$ in der Mitte auf $\xi_{x} \approx 2.5$ am Rand ausdrückt ${ }^{7}$, so daß man hoffen kann, an den Rändern weniger Sensoren als in der Mitte zu benötigen, was in Abschn. 4.3.1.1 genauer untersucht wird. Bei Annäherung an den rechten Rand wird die Verteilung der raumzeitlichen Korrelationen nach links verschoben. Dies bedeutet, daß aufgrund der hier verwendeten festen Randbedingungen die Diffusion bevorzugt Information in Richtung der Mitte transportiert. Ein Grund hierfür ist, daß die Dynamik an den Rändern stark in ihrer Freiheit eingeschränkt ist und die wandernden chaotischen Wellen sich nur von den Rändern wegbewegen können. Damit die ungekoppelten Bereiche hinreichend Information aus den gekoppelten Bereichen erhalten können, dürfen zwei Sensoren nicht mehr als $2 * \xi_{x}$ voneinander entfernt sein, welches hier einen maximalen Abstand von $d \approx 3$ entspricht. In Abb. $4.6(L=50)$ entspricht dies ca. $N=17$ Kontrollsignalen, welche auch für die Synchronisation mit der ,pinning“-Kopplung $(l=\Delta x=0.25)$ mindestens benötigt werden. Räumlich ausgedehnte Sensoren benötigen deswegen weniger Kopplungssignale, weil aufgrund ihrer Breite $l$, der Abstand ungekoppelter Bereiche (mit weniger Signalen) innerhalb der räumlichen Korrelationslänge gehalten werden kann.

Um die Stabilität der Synchronisation auch quantifizieren zu können, haben wir die sechs größten transversalen Lyapunovexponenten von Glg. (4.11) für $L=50$ und $\varepsilon=0.4$ berechnet. In Abb. 4.8a wurde die Breite $l$ bei konstanter Anzahl $N=15$ Sensoren variiert, während in Abb. 4.8b die tranversalen Lyapunovexponenten für konstante Breite $l=2.5$ in Abhängigkeit von $N$ gezeigt sind. Die beiden Bilder entsprechen den strichge-

\footnotetext{
${ }^{7}$ Bei raumzeitlich chaotischen Systemen mit periodischen Randbedingungen bleibt die Korrelationslänge $l$ entlang der gesamten räumlichen Ausdehnung konstant.
} 


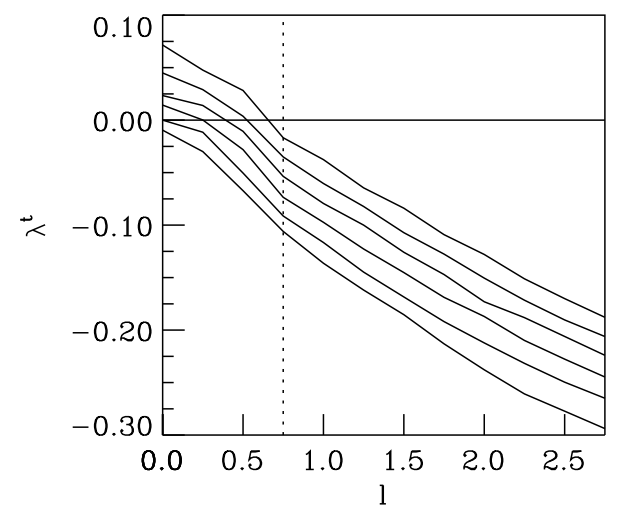

(a) $\lambda_{i}$ vs. $l, N=15$

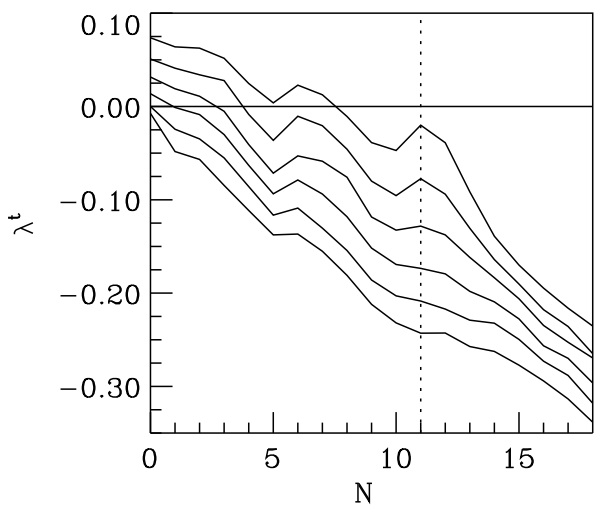

(b) $\lambda_{i}$ vs. $N, l=2.5$

Abbildung 4.8.: Die sechs größten transversalen Lyapunovexponenten von Glg. (4.11) für $L=50$ in Abhängigkeit der a) Breite $l$ und b) Anzahl der Sensoren $N$.
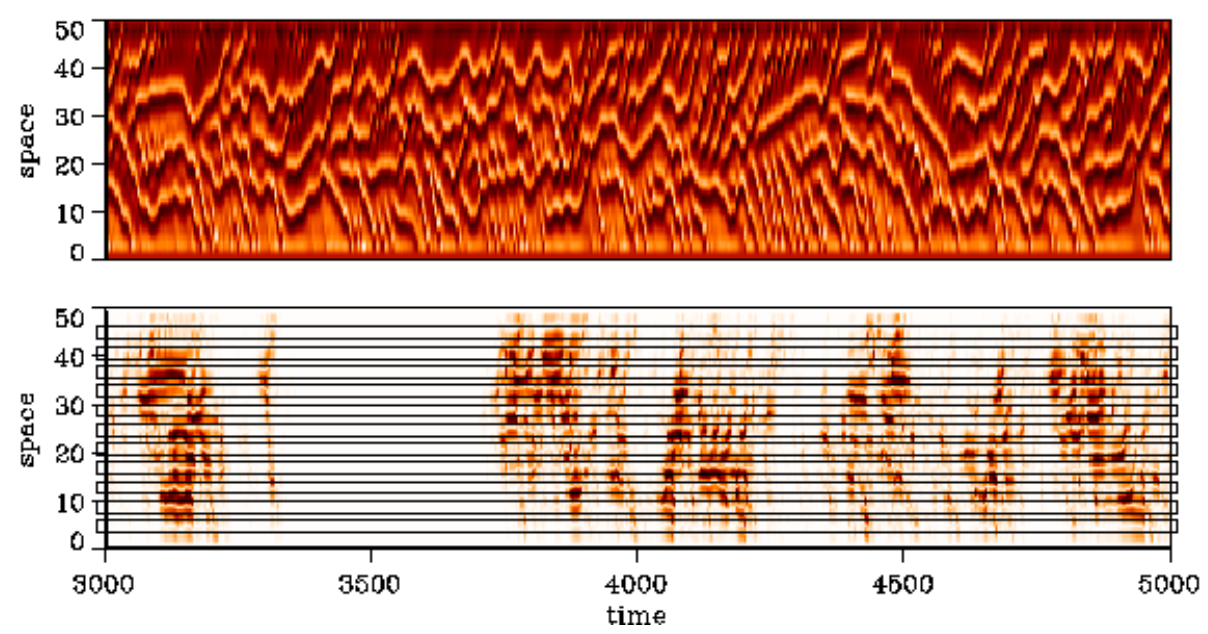

Abbildung 4.9.: On-off Intermittenz des Synchronisationsfehlers für $N=11, \varepsilon=0.4$, $l=2.5$ und $L=50$. (oben) Dynamik des treibenden Systems $u$ aus Glg. (4.10). (unten) Synchronisationsfehler $\|u-v\|$, wobei die System in den dunklen Bereichen desynchronisiert sind und in den weißen lokal Synchronisation vorliegt. Die horizontalen dünnen Rechtecke deuten die Positionen und Breite $l$ der Sensoren an.

punkteten Linien aus Abb. 4.6. Für steigendes $l$ oder $N$ nimmt die Anzahl von positiven Lyapunovexponenten ab und für $N \geq 8$ sind alle negativ. Stabile „high-quality“ Synchronisation [33] tritt jedoch erst für $N \geq 12$ auf, im Zwischenbereich von $8 \leq N<12$ wird raumzeitliche Intermittenz des Synchronisationsfehlers beobachtet [130], wo lange Zeiten von synchronisiertem Verhalten durch Intervalle von desynchronisiertem Verhal- 
ten unterbrochen sind. Abbildung 4.9 zeigt für $N=11$ Sensoren die Zeitentwicklung des treibenden Systems $u$ (oben) und des Synchronisationsfehlers $e=\|u-v\|$.

\subsubsection{Optimale Position der Sensoren}

Im letzten Abschnitt wurde die Sensoren periodisch im gesamten Raum angeordnet. Abbildung 4.7 zeigt, daß die raumzeitlichen Korrelationen zu den Rändern hin zunehmen, so daß dort möglicherweise eine geringere Dichte an Sensoren/Aktuatoren benötigt wird. Weiterhin ist es denkbar, daß durch die periodische Anordnung räumlich periodische instabile Moden nicht stabilisiert werden können, da die Sensoren unter Umständen sich hauptsächlich in der Nähe derer Nullstellen befinden. Aus diesen Gründen haben wir versucht die optimalen Positionen der Sensoren mit Hilfe von genetischen Algorithmen zu bestimmen. Für eine feste vorgegebene Anzahl von $N$ von Sensoren mit Breite $l$, wurde eine Konfiguration mit einer Kostenfunktion bewertet, welche aus dem größten transversalen Lyapunovexponenten, dem maximalen raumzeitlich lokalen Synchronisationsfehler und der Transientzeit zum synchronen Zustand aufgebaut wurde, wobei diese Größen auch in dieser Reihenfolge gewichtet waren. Beim genetischen Algorithmus sind die Rekombinations- und Mutationsregeln an das Problem angepaßt wurden, so daß die Anzahl $N$ der Sensoren konstant bleibt und sich Sensoren nicht überlappen können ${ }^{8}$ In Abb. 4.10 ist die minimale Anzahl an Kopplungssignalen $N$ zur Erreichung des syn-

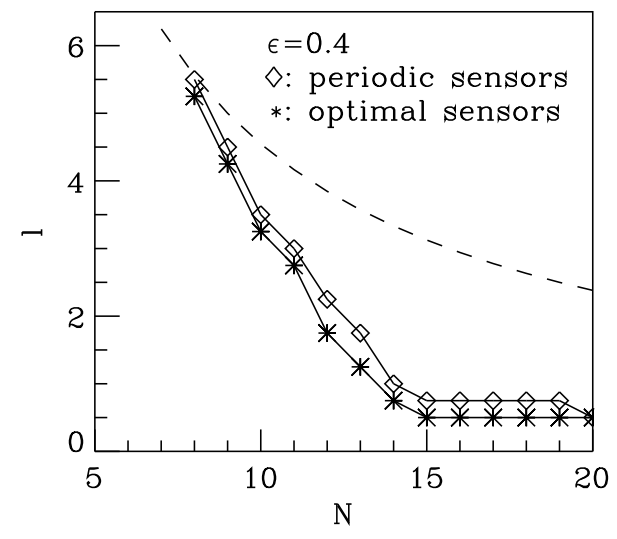

(a) $\varepsilon=0.4$

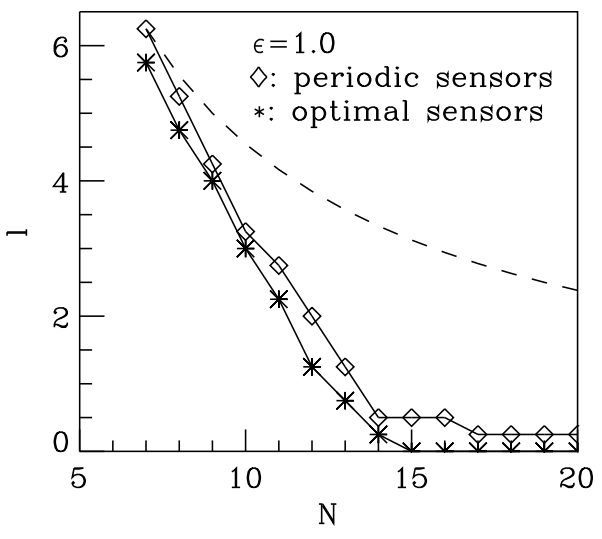

(b) $\varepsilon=1.0$

Abbildung 4.10.: Minimale Anzahl $N$ der Sensorsignale in Abhängigkeit der Breite $l$ für periodisches und optimiertes Kopplungsgitter für die Kopplungsstärke a) $\varepsilon=0.4$ und b) $\varepsilon=1.0$.

chronen Zustandes gegen die Breite $l$ der Sensoren der optimierten Kopplunganordnung

\footnotetext{
${ }^{8}$ Dies wurde notwendig da in den numerischen Standardpaketen solche Regeln leider nicht implementiert sind und der Algorithmus letztendlich dann die Anzahl der Sensoren maximiert, so daß die Sensoren schließlich den ganzen Raum bedecken.
} 
(Rauten) für die Kopplungsstärken a) $\varepsilon=0.4$ und b) $\varepsilon=1.0$ gezeigt. Zum Vergleich, sind die Graphen aus Abb. 4.6 mit periodischem Kopplungsgitter als Kreise gezeichnet. Man erkennt, daß das optimierte Kopplungsgitter nur eine leichte Reduktion der Anzahl der Sensoren gegenüber den periodischen Gitter bewirkt, so daß eine periodische Anordnung auch bei festen Randbedingungen nah am Optimum ist. Abbildung 4.11 zeigt die optimierte Anordnung der Sensoren (blau) aus Abb. 4.10b. Das optimierte Kopplungs-

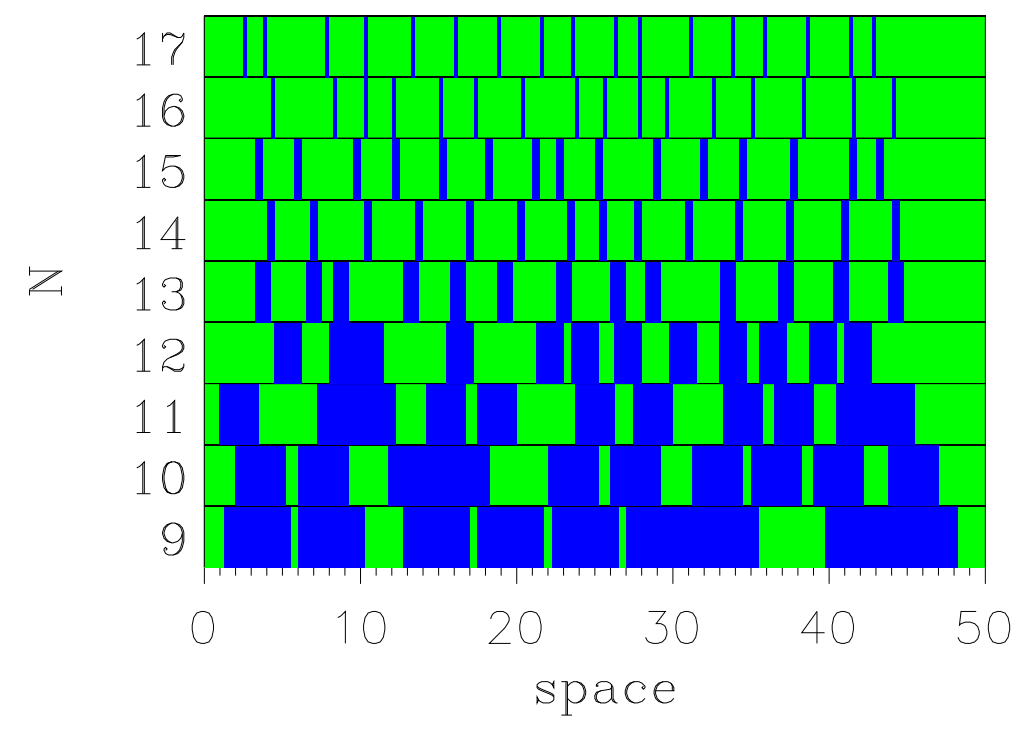

Abbildung 4.11.: Die optimierte Sensoranordnung von Abb.4.10b ist für mehrere Anzahlen $N$ von Sensoren gezeigt. Die Breite $l$ der Sensoren ist maßstabsgetreu wiedergegeben.

gitter nutzt hauptsächlich nur das regulärere Verhalten an Rändern, verursacht durch die festen Randbedingungen, aus. Im Zentrum der räumlichen Ausdehnung der PDE, wird die Anordnung annähernd periodisch, wobei nur leichte Fluktuationen auftreten, welche unter Umständen besser räumlich periodische Fouriermoden (induziert durch die endliche Länge $L$ ) unterdrücken können. Zusammenfassend ist eine streng periodische Anordnung fast optimal und sollte bei steigender räumlicher Ausdehnung der PDE noch besser werden, da dann die Rändern weiter an Einfluß auf die Dynamik verlieren.

\subsubsection{Unterdrückung von raumzeitlichem Chaos mittels Sensorkopplung}

Synchronisation und Kontrolle von Chaos sind verwandte Probleme. Die meisten Kopplungstechniken zur Synchronisation zweier Systeme können auch für Kontrollzwecke verwendet werden. Zur Demonstration benutzen wir die Sensorkopplung um raumzeitliches Chaos in einen periodischen oder stationären Zustand zu zwingen. Zur Realisierung benutzen wir in Glg. (4.9) den Ursprung $u=0$ als treibenden Zustand $\bar{u}_{n} \equiv 0, n=$ $1, \ldots, N$, welches auf den lokalen Kontrollterm $-\varepsilon \bar{v}_{n}$ führt. Der Effekt dieser Kopplung ist, daß wir den Zustand in einigen kleinen lokalen Bereichen zurückkoppeln, wodurch dort zusätzliche Dissipation eingeführt wird. Zur vollständigen Stabilisierung des 
Grundzustandes $u \equiv 0$ werden wir mehr Kontrollelemente als bei der Synchronisation benötigen, weil dieser Zustand transversal weitaus instabiler ist, wodurch der Abstand zwischen den Sensoren verringert werden muß. Dies wird später am Beispiel der Ginzburg-Landau-Gleichung in Abschn. 4.3.2.2 gezeigt werden. Hier betrachten wir eine weitere interessante Frage. Nämlich wie viele Aktuatoren notwendig sind, um raumzeitliches Chaos zu unterdrücken und eine stabile zumindest periodische Lösung zu erhalten. Bei der KS-Gleichung (4.10) mit räumlicher Länge $L=50$ benötigen wir nur ein Sensorkontrollelement der Breite $l=2.5 \mathrm{um}$ das raumzeitliche Chaos zu unterdrücken. Abbildung 4.12 zeigt die Kontrolle bei $L=50$ mit einem und fünf Sensoren. Es mag

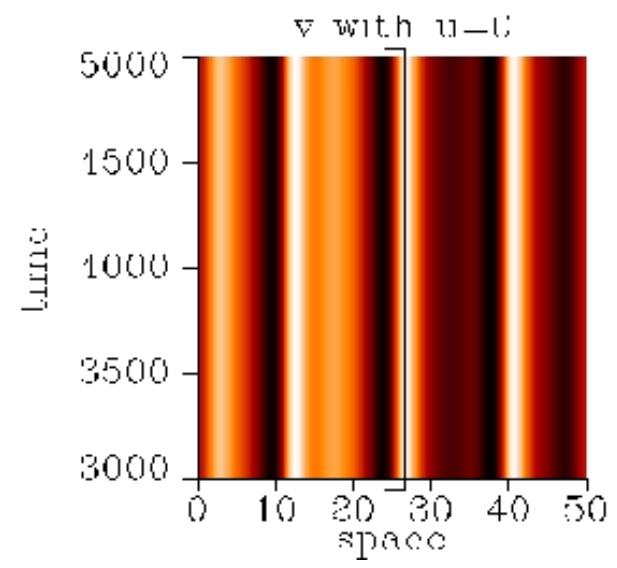

(a) 1 Sensor

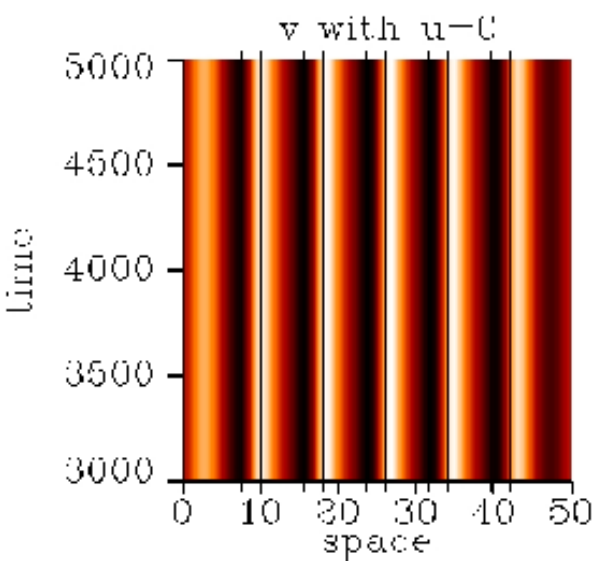

(b) 5 Sensoren

Abbildung 4.12.: Unterdrückung von raumzeitlichem Chaos von Glg. (4.10) mit periodischer Sensoranordnung. Die Parameter sind $L=50, \varepsilon=0.4, l=2.5$. In a) wurde 1 and in b) 5 Sensoren zur Kontrolle benutzt, welche durch die dünnen Rechtecke angedeutet sind.

etwas überraschen, daß man durch Einbringen von etwas Dissipation $(\epsilon=0.4)$, an nur einer lokalen Stelle, eine raumzeitlich chaotische Dynamik mit fünf positiven Lyapunovexponenten und einer Lyapunovdimension von $D_{L} \approx 10$ in ein stabiles periodisches Regime führen kann. Durch Variation der Positionen und Anzahl der Sensoren können eine Vielzahl von räumlich periodischen Mustern stabilisiert werden. Für größere Längen und damit verbundene höherdimensionale Attraktoren, z.B. für $L=200$, wird man zwar mehr als ein lokales Sensorkontrollelement zur Unterdrückung des Chaos benötigen, aber die Sensorkontrollstrategie bleibt weiterhin sehr effektiv.

\subsubsection{Intensive Untersuchung der Eigenschaften der Sensorkopplung am Beispiel der Ginzburg-Landau Gleichung}

Nachdem im letzen Abschnitt die Sensorkopplung am Beispiel der Kuramoto-Sivashinski Gleichung untersucht wurde, soll nun eine PDE mit periodischen Randbedingungen un- 
tersucht werden. Dazu verwenden wir die 1-dimensionale komplexe Ginzburg-Landau (GL) Gleichung [24, 58]

$$
\frac{\partial u}{\partial t}=\mu u+(1+i \alpha) \Delta u-(1+i \beta)|u|^{2} u, \quad u \in \mathbb{C}, \quad x \in[0, L] .
$$

Die GL-Gleichung ist eine Amplitudengleichung des komplexen Feldes $A(x, t) \in \mathbb{C}$ und

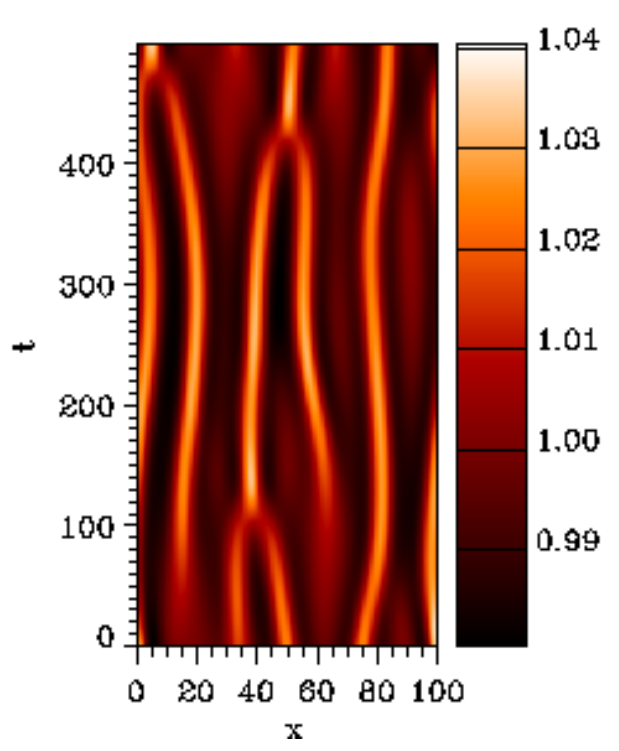

(a) Phasenturbulenz

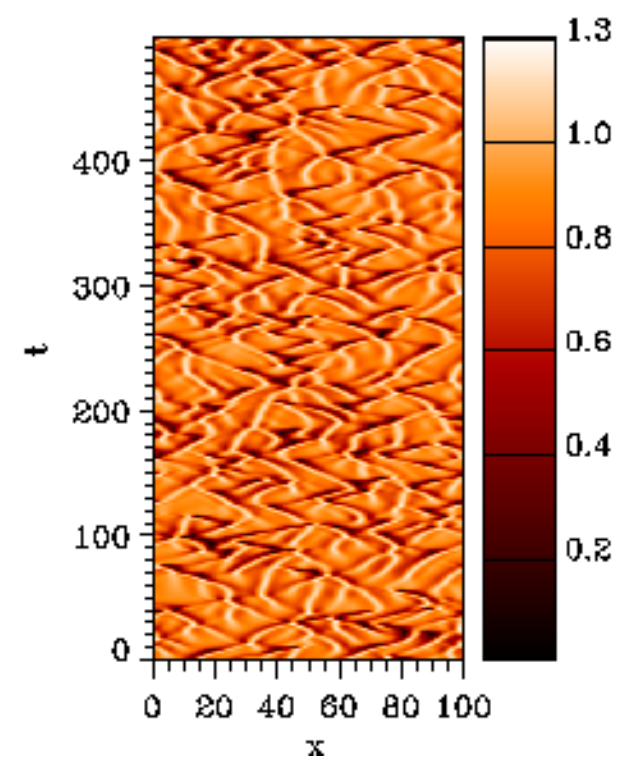

(b) Defektturbulenz

Abbildung 4.13.: Die beiden wichtigsten dynamischen Regime der Ginzburg-Landau Gleichung (4.13), wobei die Amplituden farbkodiert dargestellt sind.

beschreibt universale Strukturbildungsprozesse räumlich ausgedehnter Systeme in der Nähe einer superkritischen Hopfbifurkation, welche in der Laserphysik, Strömungsphysik, bei chemischer Turbulenz, Konvektion von binären Fluiden, etc. (siehe z.B. [75] für Referenzen), zur Beschreibung der Dynamik verwendet wird. Neben diesen Gebieten hat sich das GL-System als eine der Grundgleichungen für raumzeitliches Chaos etabliert und wird oft wegen ihrer reichhaltigen Dynamik intensiv erforscht. Gleichung (4.13) besitzt Lösungen in der Form ebener Wellen $A_{q}=\sqrt{1-q^{2}} \exp ^{i(q x-\omega t)}$ mit $q \in[-1,1]$, wobei $q$ die Wellenzahl und $\omega=-\alpha-(\beta-\alpha) q^{2}$ die Frequenz im Fourierraum beschreibt. Für $\alpha \beta>-1$ existiert ein Bereich von Wellenzahlen $-q_{c} \leq q \leq q_{c}, \quad\left(q_{c}=\sqrt{\frac{1+\alpha \beta}{2\left(1+\alpha^{2}\right)+1+\alpha \beta}}\right)$ wo diese ebenen Wellen linear stabil sind. Außerhalb dieses Bereich sind alle ebenen Wellen instabil (Eckhausinstabilität). Der Bereich der linear stabilen Wellenlösungen $q \in\left[-q_{c}, q_{c}\right]$ strebt gegen Null, wenn das Produkt $\alpha \beta-1$ erreicht, so daß oberhalb der "Benjamin-Feir" oder "Newell" Linie $\alpha \beta=-1$ keine stabilen ebenen Wellen mehr ausbreitungsfähig sind und verschiedene turbulente Zustände auftreten. Die 1-dimensionale 
GL-Gleichung (4.13) zeigt im wesentlichen drei qualitativ verschiedene turbulente dynamische Regime, Phasenturbulenz (PT) (siehe Abb. 4.13a), Defektturbulenz (DT) (siehe Abb. 4.13b) und Bichaos, wo je nach Anfangsbedingung Defekt- oder Phasenturbulenz auftritt. In diesem Abschnitt betrachten wir zwei Parametersätze, wobei $\mu=1.0, \alpha=$ 2.0, $\beta=-1.2$ zur Defektturbulenz und $\mu=1.0, \alpha=2.0, \beta=-0.7$ zur Phasenturbulenz führt (siehe Abb. 4.13). Zur chaotischen Synchronisation wenden wir an $N$ äquidistanten Orten einer identischen Kopie von Glg. (4.13)

$$
\frac{\partial v}{\partial t}=\mu v+(1+i \alpha) \Delta v-(1+i \beta)|v|^{2} v+f\left(\bar{u}_{n}, \bar{v}_{n}\right)
$$

lokale Sensorkopplungsterme $f\left(\bar{u}_{n}, \bar{v}_{n}\right)$ der Breite $l$ nach Glg. (4.9) an. Das getriebene System kann sich zwischen den Sensoren/Aktuatoren wieder frei entwickeln. Bevor wir die Eigenschaften der Sensorkopplung intensiv studieren, wollen wir zuerst ein Beispiel betrachten. In Abb. 4.14 benutzten wir $N=15$ periodisch angeordnete Sensoren der

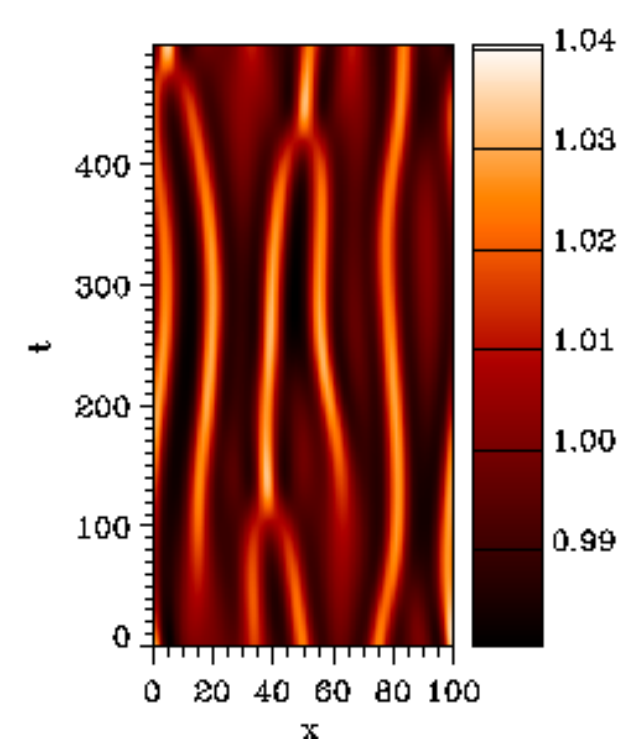

(a) Treibendes System

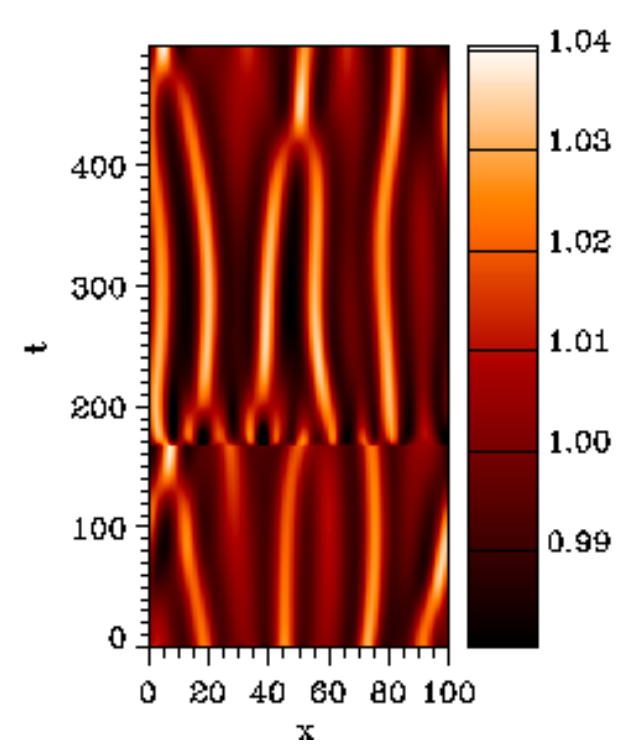

(b) Getriebenes System

Abbildung 4.14.: Synchronisation von zwei gekoppelten identischen GLEs im phasenturbulenten Regime, wozu $N=15$ Sensoren der Breite $l=3$ und Kopplungsstärke $\epsilon=0.2$ verwendet wurden.

Breite $l=3.0$ und einer Kopplungsstärke $\varepsilon=0.2$, um zwei unidirektional gekoppelte GL-Systeme der Länge $L=100$ im phasenturbulenten Regime zu synchronisieren. Die Dynamik des treibenden Systems zeigt Abb. 4.14a und in Abb. 4.14b ist die Zeitentwicklung des getriebenen Systems aufgetragen. Zum Zeitpunkt $t=170$ wurde die Kopplung aktiviert und das getriebene System konvergiert rasch auf den synchronisierten Zustand. Kurz nach der Aktivierung der Kopplung beobachtet man eine periodische Störung der 
Dynamik, welche auf die Sensorpositionen hindeuten. Kurz danach ist das getriebene System auch in den ungekoppelten Bereichen identisch synchronisiert, welche nur indirekt, durch den systemeigenen Diffusionmechanismus, Information vom treibenden System erhalten können. Nach dieser Zeit sind die beiden System ununterscheidbar und stabile Synchronisation tritt auf.

\subsubsection{Gegenseitige Abhängigkeiten der Kopplungsparameter bei der Sensorkopplung}

Im diesen Abschnitt wird systematisch und ausführlich die gegenseitige Abhängigkeit der drei Kopplungsparameter $N, l$ und $\varepsilon$ untereinander und von der Systemlänge $L$ untersucht, wobei nochmals die Ergebnisse von der KS-Gleichung bestätigt und erweitert werden. Für die Integration der PDEs (Glg. (4.13) und Glg. (4.14)) haben wir ein implizites Verfahren nach dem Crank-Nichelson Diskretisierungsschema benutzt [106], dessen Genauigkeit zweiter Ordnung im Raum und erster Ordnung in der Zeit ist. Darüberhinaus zeigt es auch für beliebige Zeitschritte keine numerischen Instabilitäten. Bei allen Integrationen wurde aus Konsistenzgründen ein Zeitschrit von $\Delta t=0.01$ und eine räumliche Gitterauflösung von 2 Gitterpunkten pro Längeneinheit der PDE benutzt. Zur Überprüfung der Lösungen haben wir einige Integrationen mit feinerer räumlicher und zeitlicher Auflösung wiederholt, wobei die Lösungen qualitativ und quantitativ keine wesentlichen Unterschiede gezeigt haben, so daß numerische Artefakte bei den weiteren Untersuchungen ausgeschlossen werden können.

Die GL-Glg. (4.13) zeigt wie die KS-Glg. (4.10) extensives Chaos. Zur Überprüfung haben wir wieder die Lyapunovdimension $D_{L}$ in Abhängigkeit der Systemlänge $L$ numerisch berechnet [73]. Das Ergebnis zeigt Abb. 4.15 für die beiden hier betrachteten Parametersätze (siehe Abb. 4.13). Die Lyapunovdimension $D_{L}$ zeigt eine exzellente lineare

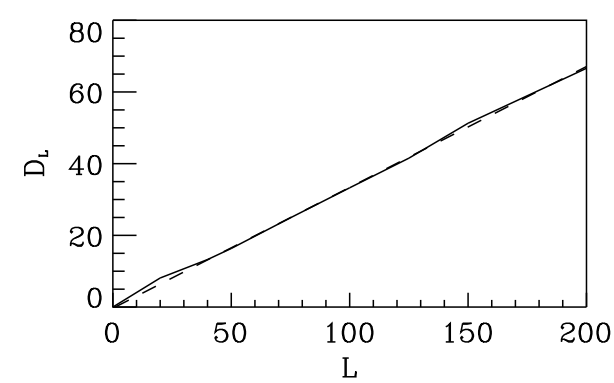

(a) $\mu=1.0, \alpha=2.0, \beta=-1.2$

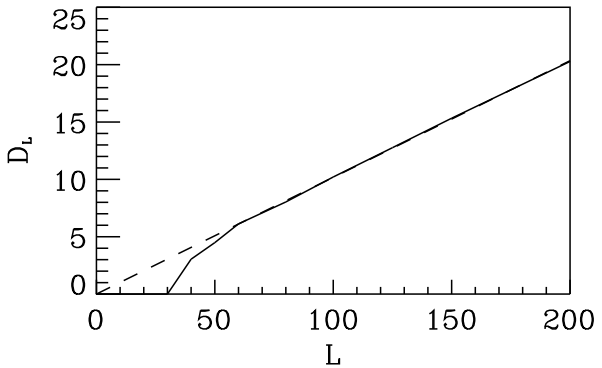

(b) $\mu=1.0, \alpha=2.0, \beta=-0.7$

Abbildung 4.15.: Lyapunovdimension $D_{L}$ gegen die Systemlänge $L$ für a) Defektturbulenz und b) Phasenturbulenz. Die gestrichelten Linien ergeben sich aus der linearen Regression.

Skalierung mit der Länge $L$ für Phasen- und Defektturbulenz, welches die Resultate von 


\section{Synchronisation und Kontrolle räumlich ausgedehnter Systeme}

Keefe [58] bestätigt ${ }^{9}$. Lineare Regression liefert die Steigungen $D_{L} \sim 0.332 L$ für Defektund $D_{L} \sim 0.102 L$ für Phasenturbulenz. Die größere Steigung bei Defektturbulenz spiegelt das weitaus komplexere fast erratische Verhalten in diesem turbulentem Regime wieder. Die folgenden Ergebnisse sind qualitativ identisch zur phasenturbulenten Dynamik, so daß wir uns im weiteren auf die Untersuchung der Sensorkopplung bei der komplexeren defektturbulenten Dynamik beschränken werden.

In Experimenten wird die Güte eines Kontrollsystems oft durch die Anzahl der benötigten Aktuatoren/Sensoren charakterisiert, weswegen wieder die minimale Anzahl $N$ der zur Synchronisation benötigten Sensoren als Indikator für die Performance der verwendeten Kopplungskonfiguration dienen soll. Abbildung 4.16 zeigt, wie die minimale Anzahl

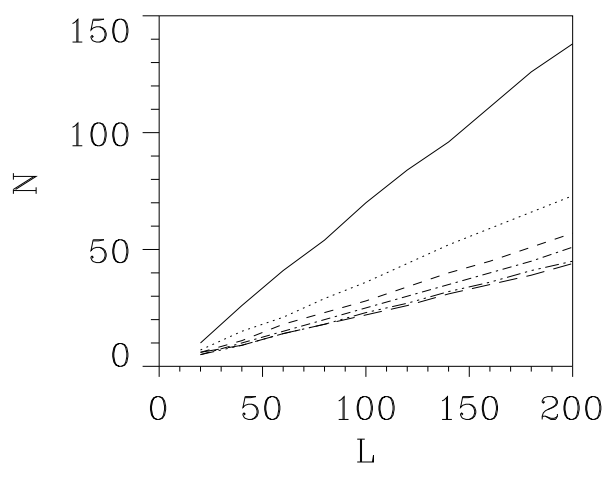

(a) $l=0.5$

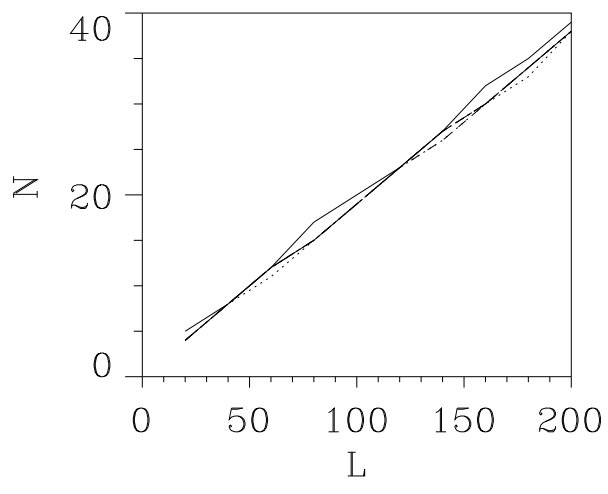

(b) $l=3.0$

Abbildung 4.16.: Minimale Anzahl $N$ von Kopplungssignalen zur Synchronisation gegen die Systemausdehnung $L$ bei jeweils fester Breite $l$ der Sensoren. Bei den einzelnen Linien nimmt die Kopplungsstärke $\varepsilon$ von oben nach unten $\varepsilon=0.5,1.0,1.5,2.0,3.0,4.0 \mathrm{zu}$.

$N$ der benötigten Sensoren mit der räumlichen Länge $L$ der GL-Gleichung, bei fester Breite $l$ und verschiedenen Kopplungsstärken $\varepsilon$, skaliert. Bei den verschiedenen Kurven in Abb. 4.16 nimmt die Kopplungsstärke von $\varepsilon=0.5$ (oben) nach $\varepsilon=4.0$ (unten) zu. Wie bei der KS-Gleichung skaliert $N$ wieder für alle Kombinationen von $l$ und $\varepsilon$ linear mit der Systemlänge $L$ und verhält sich damit wie eine extensive Größe des Systems. Somit ist, in anderen Worten, der maximal erlaubte Abstand $d$ zweier Sensoren unabhängig von $L$ und hängt nur von den weiteren Kopplungsparametern $l$ und $\varepsilon$, sowie natürlich von den speziellen Systemparametern $\mu, \alpha$ und $\beta$, ab. Der maximal erlaubte Abstand zwischen zwei Sensoren (gemessen von der Mitte der Sensoren) wird wieder für die größtmögliche Breite $l$ maximiert und bei $l \ll 1$ stimmt er, wie bei der KS-Gleichung, fast exakt mit der doppelten räumlichen Korrelationslänge $\xi_{x}$ zusammen. Damit liegt

\footnotetext{
${ }^{9}$ Die Abweichungen für kleine Längen rühren von dem Einfluß der Randbedingungen her, so daß extensives Chaos erst für $L \geq 25$ bei Defekt- und für $L \geq 50$ bei Phasenturbulenz auftritt, wo Randeffekte vernachlässigt werden können.
} 


\section{Synchronisation und Kontrolle räumlich ausgedehnter Systeme}

der Grund, warum man nicht im gesamten Raum koppeln muß, wieder in der durch die interne Diffusion verursachten Korrelation der gekoppelten mit den ungekoppelten Bereichen. In Abb. 4.16a hatten die Sensoren eine Breite von $l=0.5$, welches einem Gitterpunkt pro Sensor entspricht und damit, innerhalb der verwendeten numerischen Genauigkeit des Integrators, dem Grenzfall der „pinning“-Kopplung entspricht. Man erkennt die Beziehung, daß je größer man die Kopplungsstärke $\varepsilon$ wählt, desto weniger Kopplungssignale werden benötigt. Dies entspricht auch der Erwartung und den Erfahrungen aus niederdimensionalen Systemen, wo stärkere Kopplung meist auch bessere Performance bewirkt. In Abb. 4.16b, wo echte räumlich ausgedehnte Sensoren der Breite $l$ verwendet wurden, findet man dagegen, daß die minimale Anzahl $N$ der Kopplungssignale praktisch unabhängig von $\varepsilon$ ist und zumindest bei kleinem $\varepsilon$ weitaus weniger Signale zur Synchronisation notwendig sind. Die Erklärung für dieses Phänomen findet man in der räumlichen Ausdehnung $l$ der Sensoren. Im Grenzfall der ,pinning“-Kopplung $(l \ll 1)$ ist der Durchmesser der ungekoppelten Bereiche in der Größenordnung der doppelten räumlichen Korrelationslänge, so daß eine starke Kopplung erforderlich ist, um die Information des treibenden Systems auch in das Zentrum der ungekoppelten Bereiche zu transportieren. Bei Benutzung von räumlich ausgedehnten Sensoren mit $l \gg \Delta x$ dagegen, haben die ungekoppelten Bereiche eine kleinere Ausdehnung, so daß keine starke Kraft zum Informationstransport nötig ist. Darüberhinaus sind zur Synchronisation auch weniger Kopplungssignale notwendig, womit auch weniger Information übertragen werden muß. In Abb. 4.17 wird die Abhängigkeit von $N$ bei Variation von der Breite $l$

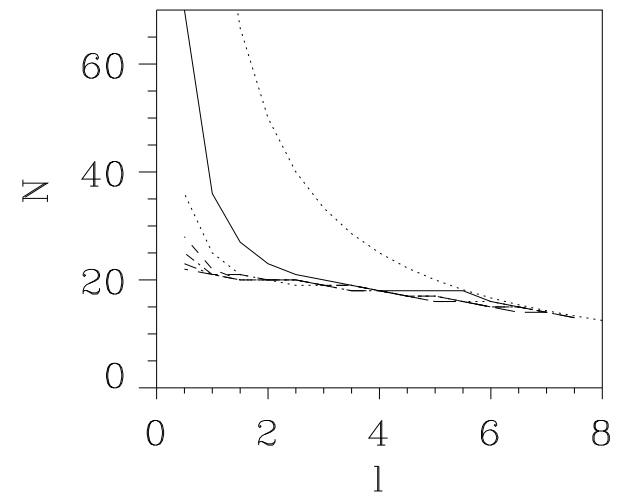

(a) $N$ vs. $l$ für $L=100$

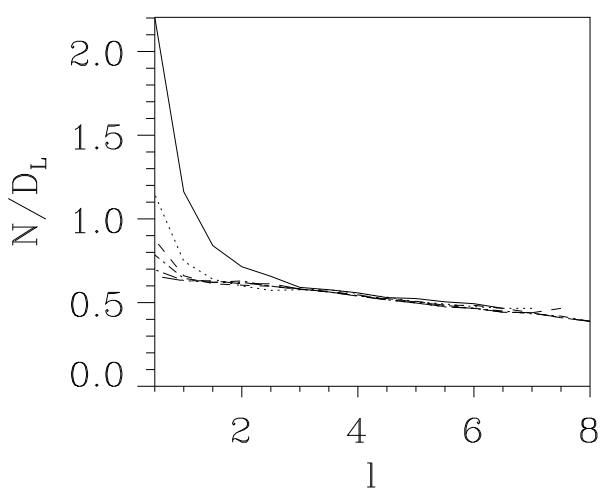

(b) $\frac{N}{D_{L}}$ vs. $l$

Abbildung 4.17.: Im linken Bild ist die minimale Anzahl von Sensoren $N$ zur Synchronisation bei der Länge $L=100$ gegen die Breite $l$ aufgetragen. Rechts ist $N$ normiert mit der Lyapunovdimension $D_{L}$ gegen $l$ gezeigt. Bei den einzelnen Linien nimmt die Kopplungsstärke $\varepsilon$ von oben nach unten $\varepsilon=0.5,1.0,1.5,2.0,3.0,4.0 \mathrm{zu}$. Die gestrichelte Linie zeigt die Grenze ab der die Sensoren sich zu überlappen beginnen.

für eine feste Systemlänge $L=100$ untersucht, wobei die Kopplungsstärke $\varepsilon$ wieder von 


\section{Synchronisation und Kontrolle räumlich ausgedehnter Systeme}

oben nach unten zunimmt. Im Grenzfall der „pinning“-Kopplung $(l=0.5)$ muß das System stark getrieben werden um eine gute Performance zu erhalten, während schon für relativ kleine Breiten $l$ die Güte der Kopplungskonfiguration praktisch unabhängig von $\varepsilon$ wird, womit die Interpration der Ergebnisse von Abb. 4.16 weiter untermauert wird. Die Benutzung von sehr breiten Sensoren bewirkt nur einen leichten Vorteil gegenüber von intermediären Werten von $l$ und wir kommen der gestrichelten Linie immer näher, wo die Sensoren sich zu überlappen beginnen und den ganzen Raum bedecken. Dies wird daran liegen, daß durch den bei der Messung durchgeführten Mittelungsprozeß bei sehr breiten Sensoren immer mehr Information vom lokalen Zustand verlorengeht, welches durch die größere gekoppelte Fläche nicht mehr wettgemacht werden kann. Im Extremfall $l \equiv L$ ist überhaupt keine Information über den lokalen Zustand im Punkt $x$ mehr vorhanden. Um nachzuweisen, das dieses Verhalten allgemein (bei festen Parametern von Glg. (4.13)) gültig ist, betrachten wir in Abb. 4.17b eine Größe welche unabhängig von der Länge $L$ ist. Da sich $N$ wie eine extensive Größe $\sim L$ verhält, können wir durch lineare Regression die Geradengleichungen aus Abb. 4.16 berechnen und erhalten durch Kombination mit der Beziehung der Lyapunovdimension $D_{L} \sim L$, die von der Länge unabhängige Größe $\frac{N}{D_{L}}$. Man kann $\frac{N}{D_{L}}$ als eine Art Dichte der Kontrollelemente ansehen, welche die minimale Anzahl der zur Synchronisation benötigten Sensoren pro Freiheitsgrad der Systemdynamik mißt. Je kleiner $\frac{N}{D_{L}}$, desto weniger Kopplungssignale werden benötigt um den Systemzustand mittels Synchronisation zu rekonstruieren, weswegen die Kopplungsparameter so gewählt werden sollten, daß diese Größe minimiert wird. Um zwei GL-Gleichungen (4.13) und (4.14) zu synchronisieren ist es ausreichend einen halben Sensor pro Attraktordimension benutzen, was die Effektivität des Verfahrens unterstreicht. Unabhängig von der jeweiligen Kopplungsstärke bewirkt die Benutzung von räumlich ausgedehnten Sensoren und Aktuatoren immer eine Reduktion der zu übertragenden Kopplungssignale, wobei bei breiten Sensoren der Einfluß von $\varepsilon$ vernachlässigbar ist, so daß bei PDEs die Sensorkopplung den „pinning"-Kopplungsvarianten, in diesem Sinne, immer überlegen ist. Der Verlauf von Abb. 4.17b ist identisch mit Abb. 4.17a, wodurch wir nachgewiesen haben, daß die Eigenschaften der Sensorkopplung unabhängig von der Systemlänge $L$ sind und nur von den Parametern der PDE abhängen. Zusammen mit den Ergebnissen aus der KS-Gleichung haben wir starke Hinweise, daß die Eigenschaften der Sensorkopplung allgemein für räumlich ausgedehnte Systeme mit extensivem Chaos gültig sind.

Eine weitere Frage ist, wie die Kopplungskonstanten die Größe der gekoppelten Fläche im Vergleich zur Gesamtlänge $L$ der PDE beeinflussen. In Abb. 4.18a ist der Prozentsatz der gekoppelten Fläche an der Systemlänge $\frac{N l}{L}$ bei festem $L=200$ gegen die Breite $l$ der Sensoren aufgetragen. In dieser Auftragung wird deutlich, daß der Preis für die Übertragung von weniger Kopplungssignalen, durch Erhöhung von $l$, in der größeren zu koppelnden Fläche liegt, so daß man in der Praxis wohl oft auf moderate Sensorbreiten $l$ zurückgreifen wird. Im rechten Teil von Abb. 4.18 ist für feste Kopplungsstärke $\varepsilon=2.0 \frac{N l}{L}$ gegen die Systemlänge aufgetragen, wobei die Breite als Parameter von unten $l=0.5$ nach oben hin $(l=8.0)$ zunimmt. Der Prozentsatz der gekoppelten Bereiche an $L$ ist damit für eine feste Kopplungskonfiguration auch unabhängig von der Systemlänge 


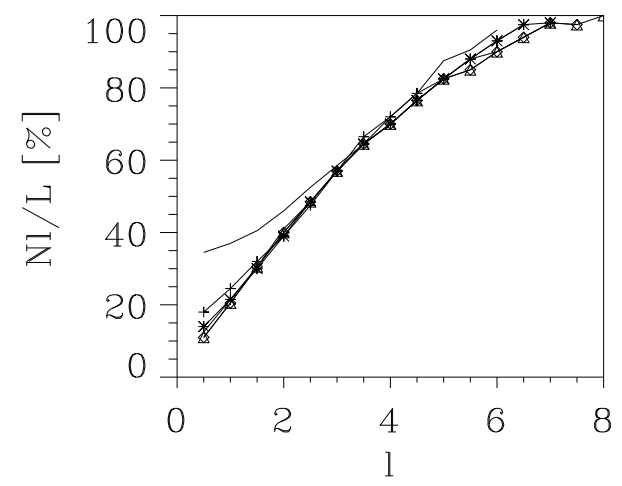

(a) $\frac{N l}{L}$ vs. $l$ für $L=200$

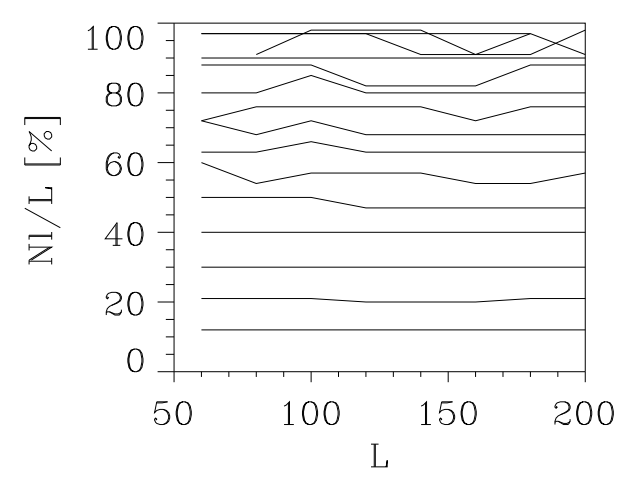

(b) $\frac{N l}{L}$ vs. $L$ für $\varepsilon=2.0$ und $l=$ $0.5, \ldots, 8.0$

Abbildung 4.18.: Anteil der Sensoren an der räumlichen Systemausdehnung $L$ in Prozent in Abhängigkeit von a) der Breite $l$ und der Systemlänge $L$. Bei den einzelnen Kurven nimmt in a) $\varepsilon=0.5,1.0,1.5,2.0,3.0,4.0$ von oben nach unten zu, während in b) $l$ von unten nach oben zunimmt.

$L^{10}$, womit auch nochmals kompakt die Unabhängigkeit des maximalen Abstandes $d$ der Sensoren (für festes $l$ und $\varepsilon$ ) von der Länge $L$ nachgewiesen worden ist.

\subsubsection{Stabilisierung des homogenen Grundzustandes $u \equiv 0$}

Die Sensorkopplungstechnik kann, wie u.A. in Abschn. 4.3.1.2 demonstriert, nicht nur zur Synchronisation, sondern auch zur Kontrolle bzw. Unterdrückung von raumzeitlichem Chaos verwendet werden. Zur Demonstration, wollen wir den Grundzustand $u \equiv 0$ von Glg. (4.13) stabilisieren. Dieser Zustand ist eine gültige Lösung von Glg. (4.13), welche aber im turbulenten Parameterbereich hochgradig instabil ist. Zur Stabilisierung fassen wir das Kontrollproblem, als Synchronisation von Glg. (4.14) mit einem treibenden System $u(x)=0, \forall x \in[0, L]$, auf. Im getriebenen System, welches ungestört Defektturbulenz zeigt, werden nun auf einem periodischen Kopplungsgitter $N$-Sensoren angeordnet, welche formal von $\bar{u}_{n}=0$ getrieben werden. Wie schon in Abschn.4.3.1.2 diskutiert, entspricht dies dem lokalen Einbringen von dissipativen Termen der Form $-\varepsilon \bar{v}_{n}$ in das Vektorfeld von Glg. (4.14). Analog zum Fall der chaotischen Synchronisation haben wir den Parameterraum der Sensorkopplung $(l$ und $\varepsilon)$ systematisch nach der minimalen Anzahl $N$, der dazu notwendigen Sensorkontrollelemente, durchsucht. Die Ergebnisse sind in Abb. 4.19 dargestellt, wobei die Darstellung äquivalent zum Synchronisationsfall in

\footnotetext{
${ }^{10}$ Die Fluktuationen von $\frac{N l}{L}$ für große Breiten $l$ sind Artefakte aus der endlichen räumlichen Diskretisierung und dem plötzlichen Auftauchen eines weiteren Kontrollelementes, während man die Länge $L$ hochfährt. Der Effekt verschwindet für sehr große Länge $L$, wo $l \ll L$ gilt, welches für kleine $l$ in Abb. 4.18b auch deutlich wird. Zum Beispiel, ist der Sprung in $\frac{N l}{L}$ durch hinzunehmen eines Sensors und $l=8$ immer noch $4 \%$, bei einer räumlichen Länge von immerhin $L=200$.
} 


\section{Synchronisation und Kontrolle räumlich ausgedehnter Systeme}

Abb. 4.17 ist. Im linken Teil von Abb. 4.19 ist die minimale Anzahl $N$ der Sensoren zur Stabilisierung von $u \equiv 0$ in Abhängigkeit von $l$ für eine feste Systemlänge von $L=100$ gezeigt, während im rechten Teil die von der Systemlänge $L$ unabhängige Größe $N / D_{L}$ in Abhängigkeit von $l$ dargestellt ist. Qualitativ gelten die gleichen Beziehungen zwischen den Kopplungsparameter wie im Synchronisationsfall in Abb. 4.17, aber nun sind deutlich mehr Kontrollellemente zum Erreichen der Zieldynamik notwendig. Der Quotient $N / D_{L}$ ist auch unabhängig von der Systemlänge, woraus ein von $L$ unabhängiger Maximalwert des Abstandes $d$ zwischen zwei Sensorkontrollelementen folgt. Der Hauptunterschied besteht darin, daß nun für jeden Freiheitsgrad des Systems (4.13) ein Kontrollelement notwendig ist, welches ungefähr dem Doppelten des Synchronisationsfalles entspricht. Dies wird einsichtig, wenn man sich klarmacht, daß der Zustand $u \equiv 0$ bei

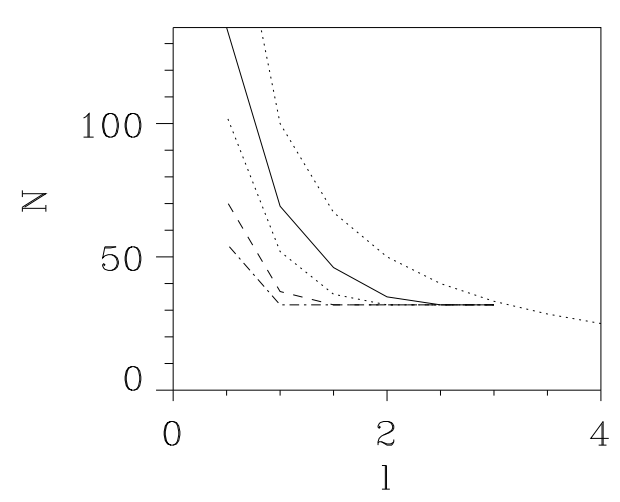

(a) $N$ vs. $l$ für $L=100$

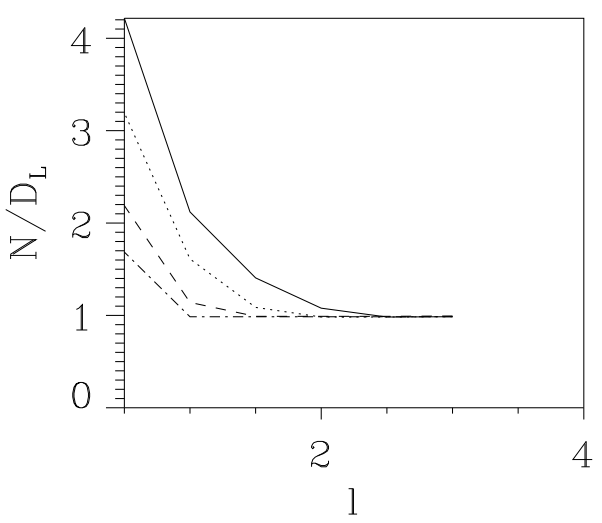

(b) $\frac{N}{D_{L}}$ vs. $l$ für $L=100$

Abbildung 4.19.: a: Minimale Anzahl $N$ der Sensoren zur Stabilisierung des Grundzustandes $u \equiv 0$ in Abhängigkeit der Breite $l$ bei festem $L=100$, wobei in b) $N$ mit der Lyapunovdimension $D_{L}$ normiert wurde. Die Kopplungsstärke $\varepsilon$ nimmt bei den Kurven von oben nach unten $(\varepsilon=$ $1.5,2.0,3.0,4.0) \mathrm{zu}$.

diesen Parametern kein Attraktor im Phasenraum ist, während der Originalattraktor eine stabile Lösung von Glg. (4.13) darstellt. Anders ausgedrückt, im getriebenen System ist $u \equiv 0$ nicht nur transversal, sondern auch tangential instabil, so daß es nicht überrascht, daß mehr Kontrollelemente zur Stabilisierung von (bzw. in dem hier gewählten Bild "Synchronisation mit") $u \equiv 0$ notwendig sind. Für Sensoren mit Ausdehnungen $l>3$ und auch für Abstände der Sensoren von $d>3$, waren wir für kein $N$ in der Lage den Grundzustand zu stabilisieren, stattdessen sind, abhängig von $\varepsilon, l$ und $N$, entweder räumlich periodische Zustände stabilisiert wurden oder lokal turbulentes Verhalten ist wieder aufgetreten. 


\subsubsection{Synchronisation und Kontrolle von raumzeitlichem Chaos in lokalen Regionen}

Nachdem wir oben Synchronisation oder Kontrolle entlang der gesamten räumlichen Ausdehnung $L$ von Glg. (4.14) erreichen wollten, wird nun demonstriert, daß das System auch nur lokal stabilisiert bzw. die gekoppelten Systeme lokal synchronisiert werden können. Da die Sensorkopplung eine räumlich lokale Kontrolltechnik ist und die Performance im wesentlichen nur vom gegenseitigen Abstand benachbarter Sensoren abhängt, ist es nicht überraschend, daß die gewünschte Zieldynamik auch nur in Teilbereichen der Systeme erreichbar ist. In Abb. 4.20 unterdrücken wir erfolgreich raumzeitliches Chaos

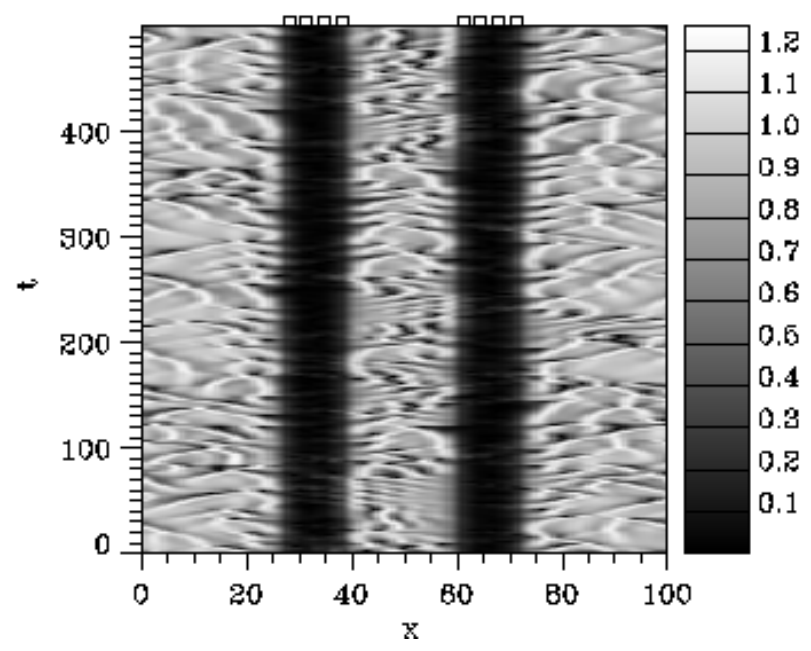

Abbildung 4.20.: Lokale Unterdrückung des raumzeitlichem Chaos in zwei Regionen, wobei jeweils 4 Sensoren der Breite $l=2.0$ mit der Kopplungsstärke $\varepsilon=2.0$ benutzt wurden. Die kleinen Rechtecke deuten wieder die Position und Breite des Sensoren an. Das Bild zeigt die farbkodierte Amplitudendynamik des kontrollierten Systems $v$ der Länge $L=100$, welches sich im defektturbulenten Regime befindet $(\mu=1.0, \alpha=2.0, \beta=-1.2)$.

in zwei Regionen, wozu wir in beiden Bereichen je 4 Sensoren mit Breite $l=2.0$ und Kopplungsstärke $\varepsilon=2.0$ eingesetzt haben. Die kleinen Rechtecke deuten wieder die Position und Breite der Sensoren an. Das Chaos konnte um das Zentrum der kontrollierten Bereiche herum vollständig unterdrückt werden. Der Rest des Systems zeigt weiterhin defektturbulente Dynamik, welche leichte Störungen an den Rändern der gekoppelten Bereiche verursacht. In Abb. 4.21 versuchen wir das getriebene System in zwei Regionen, unter Benutzung von je 6 Sensoren der Breite $l=2.0$ und Kopplungsstärke $\varepsilon=2.0$, lokal mit dem Treiber zu synchronisieren. Das obere Bild von Abb. 4.21 zeigt die farbkodierte Amplitudendynamik des getriebenen Systems $v$, im unteren Teil ist der lokale Synchronisationsfehler $e(x, t)=\|u-v\|$ farbkodiert gezeigt. In den gekoppelten Bereichen wird stabile Synchronisation beobachtet, wobei kleine Fluktuationen um Null an den Rändern auftreten, welche durch die halbseitige (interne) diffusive Kopplung mit 


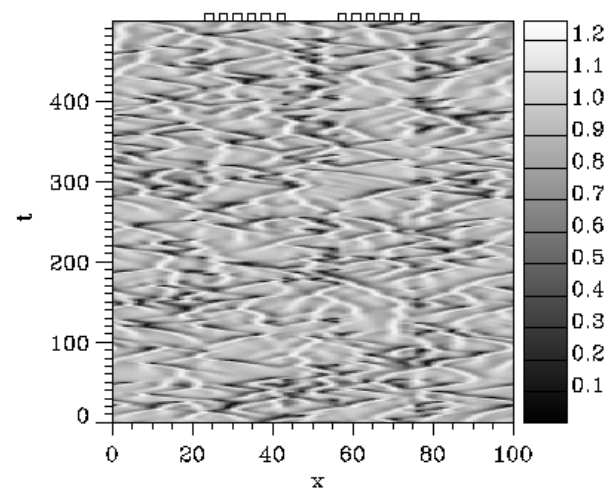

(a) $v$

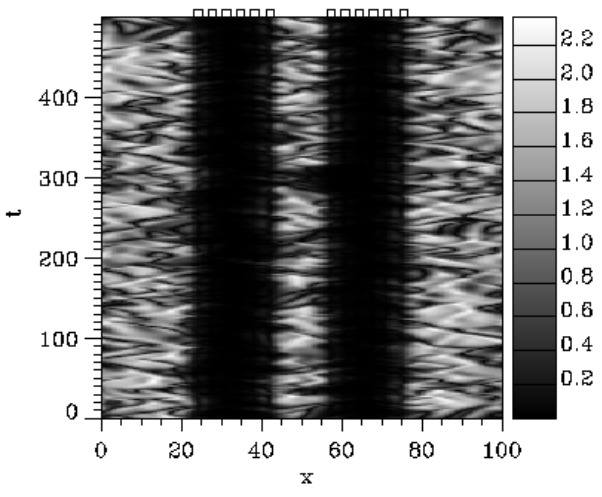

(b) $e=\|u-v\|$

Abbildung 4.21.: Lokale Synchronisation von zwei Bereichen innerhalb des raumzeitlichem Chaos mit jeweils 6 Sensoren der Breite $l=2.0$ und Kopplungsstärke $\varepsilon=2.0$. Das ungestörte System $(L=100)$ zeigt Defektturbulenz $(\mu=1.0, \alpha=2.0, \beta=-1.2)$. Links ist die farbkodierte Amplitudendynamik des getriebenen Systems und rechts der Synchronisationsfehler $e=\|u-v\|$ gezeigt.

der unsynchronisierten Eigendynamik verursacht wird.

\subsubsection{Ein paar abschließende Worte zur Sensorkopplung}

In diesem Abschnitt wurde gezeigt, wie räumlich ausgedehnte Systeme beschrieben durch partielle Differentialgleichungen miteinander synchronisiert bzw. das chaotische Verhalten unterdrückt werden kann. Wir haben ein Kopplungsschema vorgestellt, welches physikalisch sinnvoll ist und auch in realen Situationen angewendet werden kann. Die Hauptidee besteht in der bewußten Benutzung von räumlich ausgedehnten Meß- und Kontrollelementen, welche in der Wirkungsweise typischen Sensoren und Aktuatoren entsprechen. Anhand von zwei Systemen wurde gezeigt, daß der für die Synchronisation notwendige minimale Abstand der Kontrollelemente in der Größenordnung der räumlichen Korrelationslänge liegt und unabhängig von der Systemausdehnung $L$ ist. Gegenüber vorherigen Kopplungsstrategien, e.g. ,pinning“-Kopplung [37], konnte eine deutliche Reduktion der zu übertragenden Kopplungssignale erreicht werden. Die Sensorkopplung wurde erfolgreich zur globalen und lokalen Synchronisation und Unterdrückung von raumzeitlichem Chaos angewandt. Eine periodische Anordnung der Kontrollelemente wurde als praktisch optimal identifiziert. Die Kopplung sollte auch bei CMLs und PDEs mit mehrdimensionaler räumlicher Ausdehnung anwendbar sein, welches aber bis jetzt noch nicht näher untersucht wurde. Weiterhin ist auch das Konzept der zeitdiskreten Kopplung aus Abschn. 3.1.4 auf diese Kopplungstechnik übertragbar, welches nochmals eine drastische Reduktion der zu übertragenden Menge an Kopplungsinformation bewirken sollte. 


\subsection{Frequenz- und Phasensynchronisation von räumlich ausgedehnter Systeme}

Phasensynchronisation (PS) von chaotischen Systemen bei harmonischem oder chaotischem treibenden Signal ist in niederdimensionalen Systemen ein inzwischen gut verstandenes Phänomen, welches numerisch, in Experimenten und auch in der Natur inzwischen nachgewiesen werden konnte (siehe Abschn. 2.3). Aufgrund der Robustheit von PS gegenüber Störungen, wie z.B. große Parameterunterschiede, sowie additives und dynamisches Rauschen, ist zu erwarten, daß PS auch in räumlich ausgedehnten Systemen beobachtet werden kann. Kürzlich konnte PS von raumzeitlichen Chaos bei räumlich konstanten und zeitlich harmonischen treibenden Signal numerisch nachgewiesen werden [22]. In diesem Abschnitt wird untersucht, ob PS auch bei raumzeitlich chaotischen Kopplungssignalen auftreten kann.

\subsubsection{Einseitig gekoppelte Ginzburg-Landau Gleichungen}

Als Beispiel verwenden wir zwei unidirektional gekoppelte nichtidentische GinzburgLandau Gleichungen

$$
\begin{aligned}
& \frac{\partial u}{\partial t}=u+(1+i \alpha) \frac{\partial^{2} u}{\partial x^{2}}-\left(1+i \beta_{1}\right)|u|^{2} u ; \quad x \in[0, L] \\
& \frac{\partial v}{\partial t}=v+(1+i \alpha) \frac{\partial^{2} v}{\partial x^{2}}-\left(1+i \beta_{2}\right)|v|^{2} v+\varepsilon(u-v)
\end{aligned}
$$

mit periodischen Randbedingungen. Im Gegensatz zum letzten Abschnitt wird das getriebene System $v$ nun entlang der gesamten räumlichen Ausdehnung $L$ gekoppelt. Als Parameter des treibenden Systems $u$ haben wir $\alpha=2.0$ und $\beta_{1}=-0.7$ benutzt, welches zu phasenturbulenter (PT) Dynamik führt. Bei identischen Systeme tritt PS spätestens ab der Schwelle zur identischen Synchronisation auf, wo wegen $u(x, t)=v(x, t)$ die System trivialerweise auch phasensynchronisiert sind. Der interessantere Fall ist die Betrachtung von nichtidentischen Systemen, wozu wir den Parameter $\beta$ variiert und $\alpha=2.0$ vom treibenden System übernommen haben. Wir verwenden für das getriebene System zwei Parametersätze, wobei $\beta_{2}=-0.9$ zu einer im Vergleich zum treibenden System mehr turbulenten Phasendynamik und $\beta_{2}=-1.05$ auf Defektturbulenz (DT) führt. Alle Rechnungen wurden bei einer festen Systemlänge von $L=100$ durchgeführt, wo (siehe Abschn. 4.3.2) Randeffekte keinen Einfluß auf die Dynamik mehr haben. Die gekoppelten PDEs (4.15) wurden wieder mit dem impliziten Crank-Nichelson Integrationsverfahren gelöst, wobei die gleichen raum- und zeitlichen Aufösungen wie oben benutzt wurden sind.

Um zwei Systeme auf PS untersuchen zu können, wird eine Systemgröße benötigt, welche eine Phasenvariable darstellt und normalerweise mit dem Null-Lyapunovexponenten in Zusammenhang gebracht werden kann. Die Diskussion in Abschn. 2.3 hat gezeigt, daß dies selbst in niederdimensionalen Systemen ein nichtriviales Problem darstellen kann. Der Vorteil bei der GL-Gleichung ist, daß sie die Entwicklung eines komplexen Feldes beschreibt, welches formal eine eindeutige Phasendefinition $\phi(x, t)$ durch Übergang zu 
a)

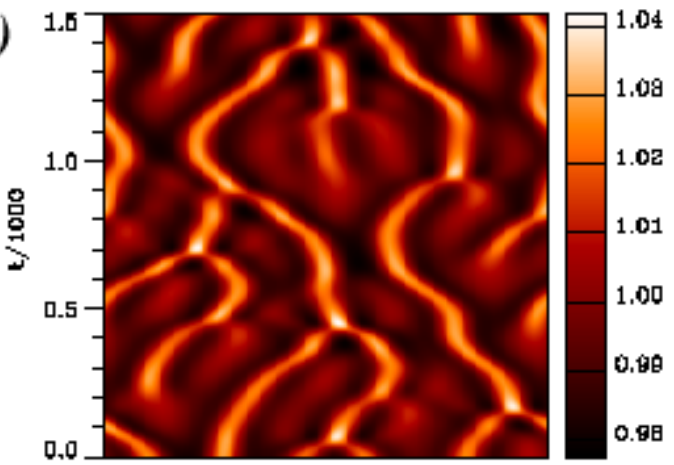

b)

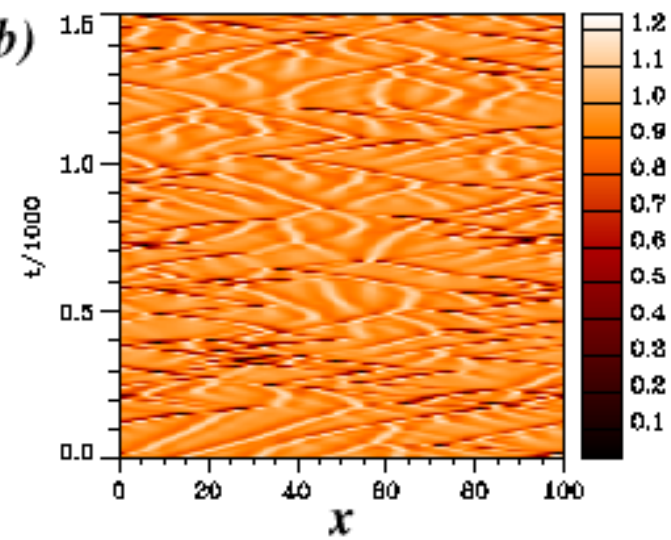

c)

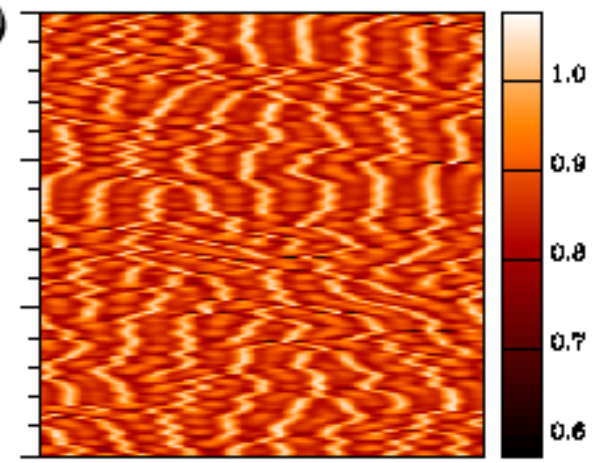

d)

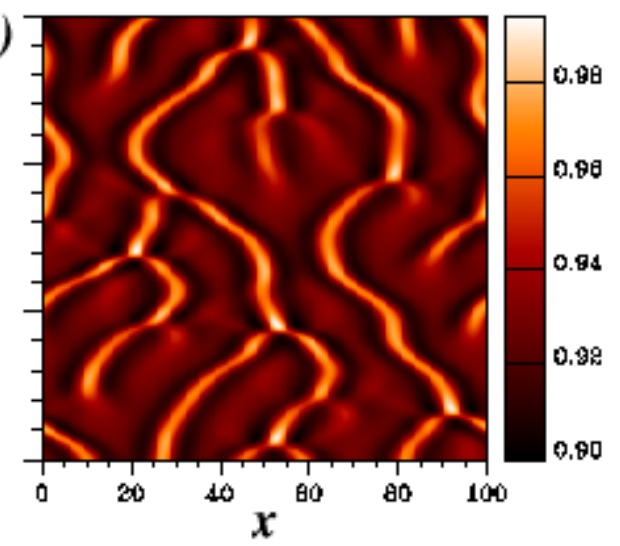

Abbildung 4.22.: Amplitudendynamik der unidirektional gekoppelten GL-Gleichungen: a) Treiber $\beta_{1}=-0.7$, b) getriebenes System $\beta_{2}=-1.05$ ohne Kopplung, c) schwache Kopplung $c=0.11$, Beginn von Phasensynchronisation, d) starke Kopplung mit $c=0.2$, starke Korrelationen der Amplitudenmuster (vergleiche (a)).

Polarkoordinaten definiert ${ }^{11}$. Die gekoppelten Systeme (4.15) betrachten wir als phasensynchronisiert [110], falls ihre Phasendifferenz im gesamten Raum nach oben beschränkt ist

$$
\left|\phi_{u}(x, t)-\phi_{v}(x, t)\right|<\text { konst.; } \quad \forall x \in[0, L], \forall t>T,
$$

wobei $T$ die Transiente bezeichnet. Die mittlere Frequenz des Systems kann mit $\phi(x, t)$ als

$$
\Omega=\lim _{t \rightarrow \infty} \frac{<\phi(x, t)>_{x}}{t},
$$

\footnotetext{
${ }^{11} \mathrm{Ob}$ in Polarkoordinaten $\phi(x, t)$ eine physikalisch sinnvolle Phase beschreibt, kann auch hier nicht immer garantiert werden. Innerhalb von Defekten $\|u(x, t)\|=0$ macht der Begriff einer Phase physikalisch keinen Sinn mehr. Dies ist ein Grund, weswegen wir das treibende System im phasenturbulenten Regime gewählt haben, wo Defekte ausgeschlossen werden können und die Phase global wohldefiniert berechnet werden kann.
} 


\section{Synchronisation und Kontrolle räumlich ausgedehnter Systeme}

definiert werden, wobei $\left\langle>_{x}\right.$ für die Mittelung über den Raum steht. Phasensynchronisation impliziert das Verschwinden der Differenz

$$
\Delta \Omega=\Omega_{u}-\Omega_{v}=0
$$

der mittleren Frequenzen $\Omega_{u}$ und $\Omega_{v}$, so daß auch Frequenzsynchronisation (FS) auftreten muß. Das Gegenteil ist, wegen möglicher seltener Phasensprünge, i.A. nicht richtig, so daß beim Hochfahren der Kopplung typischerweise das Szenario, wo zuerst die mittleren Frequenzen und danach die Phasen synchronisieren, beobachtet wird. Exakte Phasensynchronisation verbietet das Auftreten von Phasensprüngen gänzlich, welche aufgrund des Mittlerungsprozesses und bei seltenen Auftreten bei Frequenzsynchronisation nicht ins Gewicht fallen müssen. Zum Nachweis von PS mit Glg. (4.16) müssen die auf der

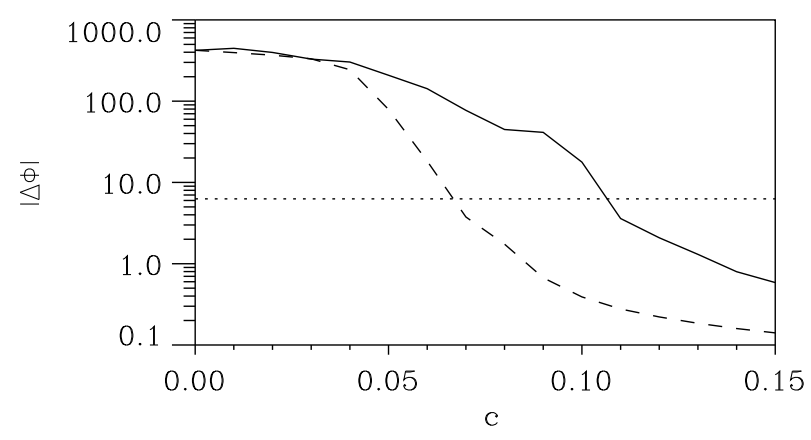

Abbildung 4.23.: Relative Phasendifferenz (4.19) vs. Kopplungsstärke $c$. Die durchgezogene Linie entspricht $\beta_{2}=-1.05$ (DT) und die gestrichelte $\beta_{2}=-0.9$ (PT). Unterhalb der gepunkteten Linie ist die Phasendifferenz kleiner als $2 \pi$, so daß Phasensynchronisation auftritt.

ganzen reellen Achse $\mathbb{R}$ fortgesetzten Phasen $\phi(x, t) \in \mathbb{R}$ verwendet werden, weil das Langzeitverhalten der Phasendifferenz entscheidend ist. Bei zyklischen Phasen $\tilde{\phi}$ dagegen können keine Phasensprünge, z.B. von $2 \pi$, welche in Defekten auftreten können und die Beschränktheit in Glg. (4.16) zerstören, nachgewiesen werden. Um eine quantitatives Maß für PS bei räumlich ausgedehnten Systemen zu besitzen, berechnen wir die maximale relative Phasendifferenz der fortgesetzten Phasen $\phi_{u}$ und $\phi_{v}$

$$
\Delta \phi=\max _{x \in L, T \leq t \in \mathbb{R}}\left|\phi_{u}(x, t)-\phi_{v}(x, t)\right| .
$$

Nach einer Transiente $T$, setzen wir die Anfangsphasendifferenz zu $\left|\phi_{u}(x, 0)-\phi_{v}(x, 0)\right| \leq$ $\pi^{12}$ und für perfekte PS kann die Phasendifferenz $\Delta \phi$ diese Grenze niemals mehr überschreiten. Die maximale relative Phasendifferenz $\Delta \phi$ nach 2500 Zeiteinheiten ist in Abb. 4.23 gegen die Kopplungsstärke $c$ aufgetragen, wobei die durchgezogene Linie das defektturbulente $\left(\beta_{2}=-1.05\right)$ und die gestrichelte Line das phasenturbulente $\left(\beta_{2}=\right.$ $-0.9)$ getriebene System beschreibt. Die gepunktete Linie zeigt die Grenze von $2 \pi$ für PS.

\footnotetext{
${ }^{12}$ Dies ist erlaubt, weil auch bei den fortgesetzten Phasen $\phi_{u, v}$ die Anfangsphase $\phi(x, 0)$ nur bis auf Vielfache von $2 \pi$ bestimmt ist.
} 


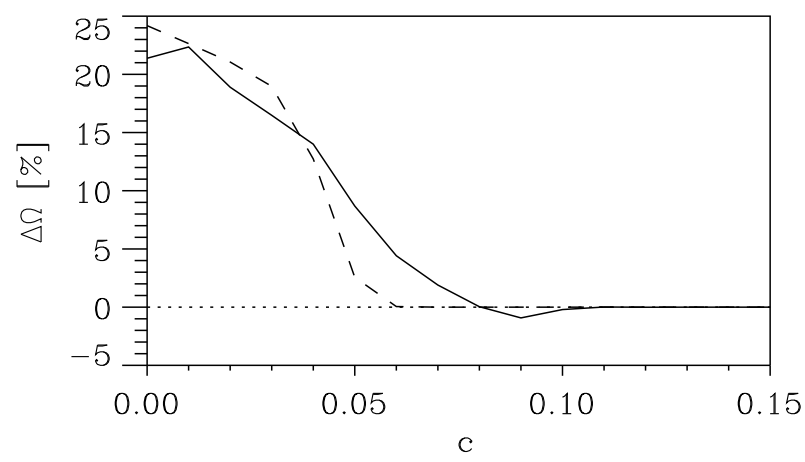

Abbildung 4.24.: Relativer Frequenzunterschied $\Delta \Omega$ gegen die Kopplungsstärke $c$. Die durchgezogene Linie entspricht $\beta_{2}=-1.05$ (DT) und die gestrichelte $\beta_{2}=-0.9(\mathrm{PT})$.

Die Phasendifferenz nimmt mit steigender Kopplungsstärke monoton ab. Für den Fall $\beta_{2}=-0.9$ (Phasenturbulenz) tritt der Übergang zu PS bei $c=0.07$ und bei $\beta_{2}=-1.05$ (Defektturbulenz) bei $c=0.11$ auf. Zur Visualisierung ist in Abb. 4.22 bei ausgewählten Kopplungsstärken $c$ die Amplitudendynamik $\|v\|$ des getriebenen Systems gezeigt. Abbildung 4.22a zeigt die raumzeitliche Dynamik des treibenden Systems $\left(\beta_{1}=-0.7\right)$, in Abb. 4.22b ist das getriebene System $\left(\beta_{2}=-1.05\right)$ ohne Kopplung $c=0$, in Abb. 4.22c kurz nach dem Übergang zur PS $c=0.11$ und in Abb. 4.22d bei starker Kopplung $c=0.20$ aufgetragen. Analog zur PS bei niederdimensionalen Systemen, impliziert PS keine Abhängigkeiten der Amplituden der gekoppelten Systeme, welches Abb. 4.22c deutlich zeigt. Erst für starke Kopplung werden auch die Amplitudenmuster stark korreliert und $\mathrm{ab} c=0.2$ (Abb. 4.22d) sogar praktisch identisch, welches ein Indiz für eine stärkere Form von chaotischer Synchronisation darstellt. Abbildung 4.24 zeigt den mittleren Frequenzunterschied (Glg. (4.17)) in Abhängigkeit von der Kopplungsstärke $c$. Wie erwartet, tritt im phasensynchronisierten Bereich auch Frequenzsynchronisation auf und die mittleren Frequenzen synchronisieren kurz bevor den Phasen, so daß folgendes Szenario beobachtet wird

$$
\begin{aligned}
& \beta_{2}=-0.90 \quad: \quad c \geq 0.06 \Rightarrow F S, \quad c \geq 0.07 \Rightarrow P S \\
& \beta_{2}=-1.05 \quad: \quad c \geq 0.10 \Rightarrow F S, \quad c \geq 0.11 \Rightarrow P S .
\end{aligned}
$$

In dem intermediären Regime treten Phasensprünge auf, welche fast vollständig auf Defekte zurückzuführen sind. Sie erfolgen aber so selten, daß sie nur einen verschwindenden Einfluß auf die mittlere Frequenz $\Omega_{2}$ ausüben. Das Auftreten von PS wurde in Abb. 4.23 direkt über die Definition Glg. (4.16) exakt nachgewiesen. Eine alternativer Weg wurde in $[83,118,131]$ vorgeschlagen und besteht in der Untersuchung der Statistik der zyklischen Phasendifferenz

$$
\widetilde{\Delta \phi}=\widetilde{\phi_{u}}(x, t)-\widetilde{\phi_{v}}(x, t) \in[-\pi, \pi]
$$

mit $\widetilde{\phi}_{i}=\phi_{i} \bmod 2 \pi \in S^{1}$ (Einheitskreis). Zum Nachweis von FS ist es hinreichend eine ausgeprägte Spitze in der Verteilung der zyklischen Phasen $\widetilde{\Delta \phi}$ nachzuweisen, welches 

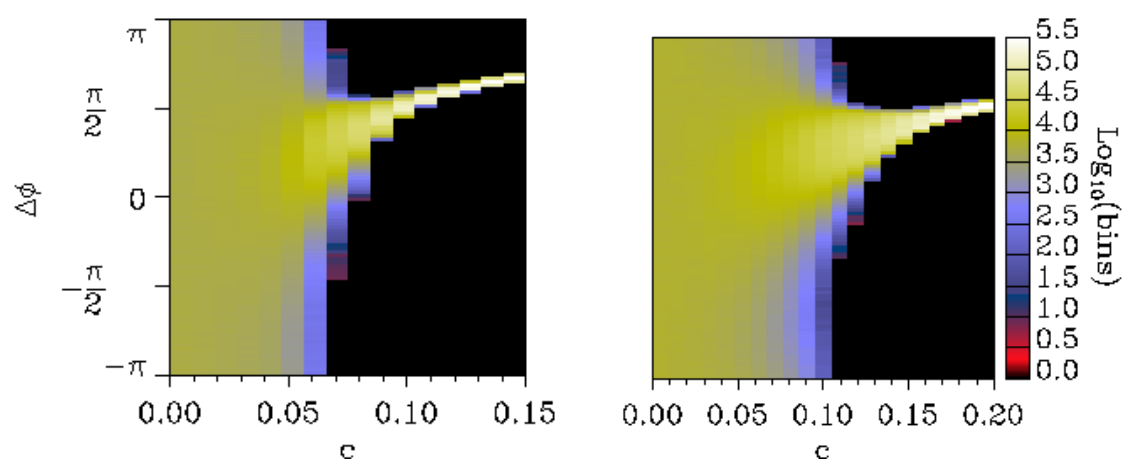

Abbildung 4.25.: Histogramm der relative zyklischen Phasendifferenz (4.21) gegen die Kopplungsstärke $c$. Die durchgezogene Linie entspricht $\beta_{2}=-1.05$ (DT) und die gestrichelte $\beta_{2}=-0.9$ (PT). Der Logarithmus des Boxinhaltes ist farbkodiert gezeigt.

gleichbedeutend ist, daß die Systeme die meiste Zeit eine feste Phasendifferenz aufweisen, woraus gleiche mittlere Umlauffrequenzen folgen. Um zusätzlich mit dieser Methode auch perfekte PS (ohne Phasensprünge) nachweisen zu können, muß das Histogramm der zyklischen Phasendifferenz auf eine Untermenge des Einheitskreises mit Durchmesser $d(\{\widetilde{\Delta \phi}\})<2 \pi$ beschränkt bleiben, d.h. es erscheint eine Lücke im Histogramm. Dieses Argument bleibt nur im rauschfreien Fall gültig, weil typischerweise, das in Experimenten unvermeidbare, (Gauß'sche) Rauschen Phasensprünge verursacht, wodurch die Lücke im Histogram geschlossen wird, so daß rauschinduzierte Phasensprünge nicht von echten unterschieden werden können. Das Verfahren ist damit zum Nachweis von FS uneingeschränkt anwendbar, kann aber streng genommen nur bei praktisch unverrauschten Zeitreihen PS exakt detektieren. Bei (realen) verrauschten Signalen können nur statistische Aussagen über PS gemacht werden, wodurch immer eine gewisse Unsicherheit übrigbleibt. In unserem betrachteten (idealisierten) rauschfreien Fall kann aber die Methoden zum sicheren Nachweis von FS und PS verwendet werden. Abbildung 4.25 zeigt die Histogramme von $\widetilde{\Delta \phi}$ in den beiden untersuchten Regimen in Abhängigkeit von $c$. Wir haben den Logarithmus des Boxinhaltes aufgetragen, um deutlich das erste Auftreten der Lücke nachweisen zu können. Im ungekoppelten Fall ist $\widetilde{\Delta \phi}$ im Einheitskreis $[-\pi, \pi]$ gleichverteilt. Bei steigender Kopplung $c$ taucht ein am Anfang noch wenig ausgeprägtes Maximum auf, welches die Tendenz der Systeme zur Synchronisation ihrer Phasen/Umlauffrequenzen darstellt (das Maximum ist für kleine $c$ in der logarithmischen Skalierung in Abb. 4.25 nicht deutlich erkennbar). An der Schwelle zur FS wird die Verteilung deutlich schmaler, bedeckt aber noch den vollen Einheitskreis $[-\pi, \pi]$, welches das Auftreten von (seltenen) Phasensprüngen nahelegt. Eine Lücke taucht im linken Bild $\left(\beta_{2}=-0.9\right)$ bei $c=0.07$ und im rechten $\left(\beta_{2}=-1.05\right)$ bei $c=0.11$ auf, welches exakt mit dem in Abb. 4.23 und Glg. (4.20) beobachteten Übergang zu PS übereinstimmt. Weitere Erhöhung der Kopplungsstärke $c$ führt zu einer deltapulsförmigen Verteilung um eine feste gegenseitige relative Phasendifferenz.

Für starke Kopplung $c$ werden in Abb. 4.22d die Amplitudenmuster des getriebenen Sy- 


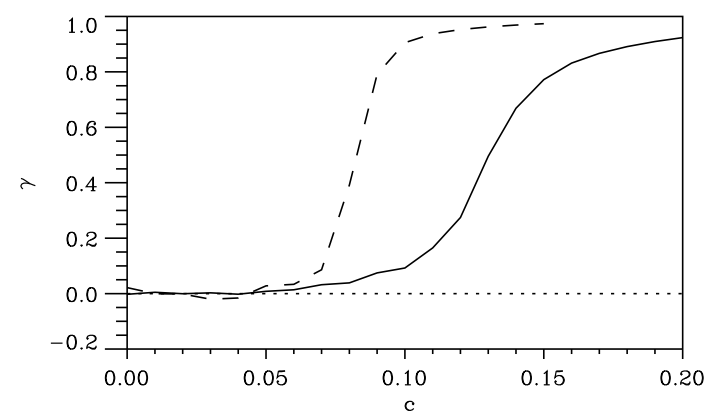

Abbildung 4.26.: Linearer Korrelationskoeffizient gegen die Kopplungsstärke $c$. Die durchgezogene Linie entspricht $\beta_{2}=-1.05$ (DT) und die gestrichelte $\beta_{2}=-0.9(\mathrm{PT})$.

stems, denen des treibenden in Abb. 4.22a, sehr ähnlich, während für intermediäre Werte trotz PS keine Ähnlichkeiten zwischen $\|u\|$ und $\|v\|$ erkennbar sind. Um dies zu quantifizieren haben wir den linearen Produkt-Moment Korrelationskoeffizienten $\gamma$ (auch als Pearson's $r$ bekannt) zwischen den raumzeitlichen Amplitudenmustern $\|u\|$ und $\|v\|$ in Abhängigkeit von $c$ berechnet, welcher in Abb. 4.26 dargestellt ist.

$$
\gamma=\frac{\sum_{i}\left(\left|u_{i}\right|-\bar{u}\right)\left(\left|v_{i}\right|-\bar{v}\right)}{\sqrt{\sum_{i}\left(\left|u_{i}\right|-\bar{u}\right)^{2}} \sqrt{\sum_{i}\left(\left|v_{i}\right|-\bar{v}\right)^{2}}}
$$

wobei $\bar{u}, \bar{v}$ die Mittelwerte von $|u|,|v|$ sind und $-1 \leq \gamma \leq 1$ gilt. Für einen Wert von $\gamma$
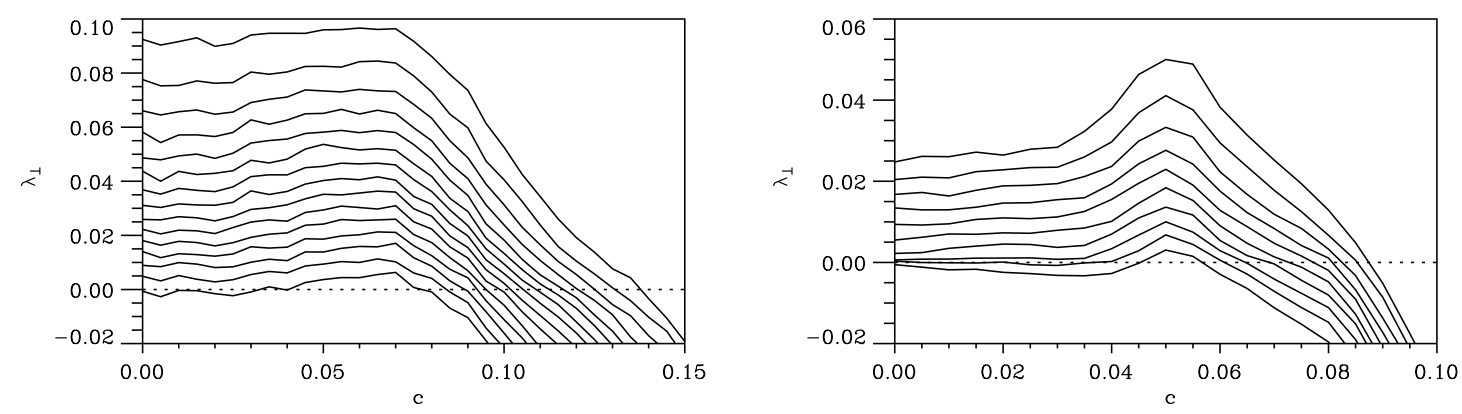

Abbildung 4.27.: Größter transversaler Lyapunovexponent $\lambda_{\perp}$ gegen die Kopplungsstärke $c$. a) $\beta_{2}=-1.05$ (DT), b) $\beta_{2}=-0.9$ (PT).

um Null sind die Muster linear unkorreliert, für $\gamma=1$ vollständig positiv korreliert und für $\gamma=-1$ antikorreliert. Für beide betrachteten Regime $\beta_{2}=-1.05$ und $\beta_{2}=-0.9$ sind die Amplitudenmuster bei Einsetzen von PS $c=0.11$ (0.07) vollständig unkorreliert, siehe auch Abb. 4.22c für $\beta_{2}=-1.05$. Weitere Erhöhung der Kopplungsstärke induziert stärkere Korrelationen, welche bei starker Kopplung auf praktisch identische Dynamik hindeuten. Man beachte, daß die Systeme keine identische Synchronisation 
zeigen können, da sie nichtidentisch $\beta_{1} \neq \beta_{2}$ sind, so daß eine invariante Synchronisationsmannigfaltigkeit der Form $u \equiv v$ nicht existiert. In Abschn. 2.2 wurde diskutiert, daß stattdessen bei nichtidentischen Systemen, z.B. bei Parameterunterschieden, verallgemeinerte Synchronisation (GS) [61, 114] auftreten kann, so daß ein funktionaler Zusammenhang zwischen $u$ und $v$ angenommen wird. Eine notwendige Bedingung für GS ist, daß alle transversalen Lyapunovexponenten negativ sind $\lambda_{\perp}^{\max }<0$. Zur Überprüfung sind in Abb. 4.27a (b) die 15 (9) größten transversalen Lyapunovexponenten des getriebenen Systems für $\beta_{2}=-1.05\left(\beta_{2}=-0.9\right)$ in Abhängigkeit der Kopplungsstärke $c$ bei beiden Parametersätzen aufgetragen. Beim Einsetzen von PS bei $c=0.11(c=0.07)$ sind weiterhin 6(6) positive Lyapunovexponenten vorhanden, so daß kein GS auftreten kann. Das getriebene System besitzt damit noch 6 eigene angeregte Freiheitsgrade, weswegen die Amplitudenmuster auch keine starken linearen Korrelationen aufweisen können, siehe Abb. 4.26. Dagegen sind für $c=0.15(c=0.09)$ alle transversalen Lyapunovexponenten negativ, so daß das getriebene System nun „versklavt" ist und (zumindest im Mittel) dem Treiber folgen muß. Dieser Übergang ist mit einem starken Sprung des linearen Korrelationskoeffizienten $\gamma$ auf Werte nahe Eins verbunden, welches ein weiteres Indiz für das Auftreten einer festen funktionalen Beziehung zwischen den Flüssen der gekoppelten Systeme liefert. Somit beobachten wir im wesentlichen das gleiche Szenario, wie bei niederdimensionalen Systemen, wo bei steigender Kopplung der Übergang von unsynchronisiertem Verhalten, über Frequenzsynchronisation, Phasensynchronisation schließlich bei stark gekoppelten Systemen auf verallgemeinerte Synchronisation führt.

\subsubsection{Beidseitig gekoppelte Ginzburg-Landau Gleichungen}

Während im letzten Abschnitt die Phasen/Frequenzsynchronisation räumlich ausgedehnter unidirektional gekoppelter Systeme untersucht wird, soll im folgenden die gleiche Analyse bei bidirektional gekoppelten Systemen wiederholt werden. Der Übergang von uni- zu bidirektionaler Kopplung wird an der reinen Existenz eines phasensynchronisierten Zustandes qualitativ nichts ändern, siehe auch Abschn. 3, wo die lokale Äquivalenz von uni- und bidirektionaler Kopplung [52] diskutiert wurde. Bei der kritischen Kopplungsstärke, ab der FS/PS auftritt, können aber durchaus weitgehende Unterschiede bestehen. Kürzlich wurde von Bocaletti et.al. [12] zwei bidirektional gekoppelte nichtidentische GL-Gleichungen

$$
\begin{aligned}
& \frac{\partial u}{\partial t}=u+\left(1+i \alpha_{1}\right) \frac{\partial^{2} u}{\partial x^{2}}-\left(1+i \beta_{1}\right)|u|^{2} u+c(v-u) ; \quad x \in[0, L] \\
& \frac{\partial v}{\partial t}=v+\left(1+i \alpha_{2}\right) \frac{\partial^{2} v}{\partial x^{2}}-\left(1+i \beta_{2}\right)|v|^{2} v+c(u-v)
\end{aligned}
$$

mit periodischen Randbedingungen untersucht. Die Parameterwerte der ungekoppelten Systeme $c=0$ führen auf Defektturbulenz $\alpha_{1}=2.1$ und $\beta_{1}=-1.2$ und Phasenturbulenz $\alpha_{1}=2.1$ und $\beta_{1}=-0.83$. Die Autoren berichten von einem Übergang von unabhängiger Dynamik über PS $(0.1 \leq c \leq 0.16)$ zu identischer Synchronisation. Wie im letzten Abschnitt diskutiert, macht es bei nichtidentischen Systemen keinen Sinn von 


\section{Synchronisation und Kontrolle räumlich ausgedehnter Systeme}

identischer Synchronisation zu sprechen. Es kann maximal eine sehr ähnliche Dynamik bei sehr starker Kopplung erzwungen werden, welche aber nur für $c \rightarrow \infty$ zur Existenz und dem Auftreten von identischer Synchronisation führen kann. Weit schwerer wiegen die Ausführungen über PS. Zum Nachweis werden die über Raum und Zeit gemittelte Amplituden- $\Delta \rho=\langle|u-v|\rangle_{x, t}$ und zyklische Phasendifferenz $\widetilde{\Delta \psi}=\left\langle\left|\widetilde{\phi_{u}}-\widetilde{\phi_{v}}\right|\right\rangle_{x, t}$ verwendet. Als ein Kriterium für PS wird das Auftreten eines Plateaus von $\widetilde{\Delta \psi}$ bei Variation der Kopplungsstärke $c$ vorgeschlagen. Als zweites Kriterium wird die gleiche Anzahl von Defekten $N_{d}$ in beiden Systemen (4.23) (welche in einer hinreichend großen Zeitspanne gemessen wurden) als Indikator für PS benutzt. Beide Kriterien sind nicht zum Nachweis von PS geeignet. Dies kann unten, durch Anwendung der von uns benutzten Nachweismethoden bei Wiederholung der Simulationen von [12], auch gezeigt werden. Zuerst wollen wir die beiden Kriterien aus [12] näher betrachten. Bei der Berechnung von $\widetilde{\Delta \psi}=\left\langle\left|\widetilde{\phi_{u}}-\widetilde{\phi_{v}}\right|\right\rangle_{x, t}$ werden nicht die für PS Glg. (4.19) entscheidenden fortgesetzten Phasen $\phi$, sondern die zyklischen Phasen $\widetilde{\phi}$ verwendet, welche z.B. insensitiv gegenüber Phasensprüngen von $2 \pi$ sind, die in Defekten $u=0$ auftreten können. Weiterhin macht man sich leicht klar, daß im ungekoppelten Fall $c=0$ die mittlere Phasendifferenz für beliebige Systeme und Parameter immer verschwinden $\widetilde{\Delta \psi}=0$ muß, da auch die mittleren Phasen der Einzelsysteme verschwinden. Phasensynchronisation ist eine intrinsische
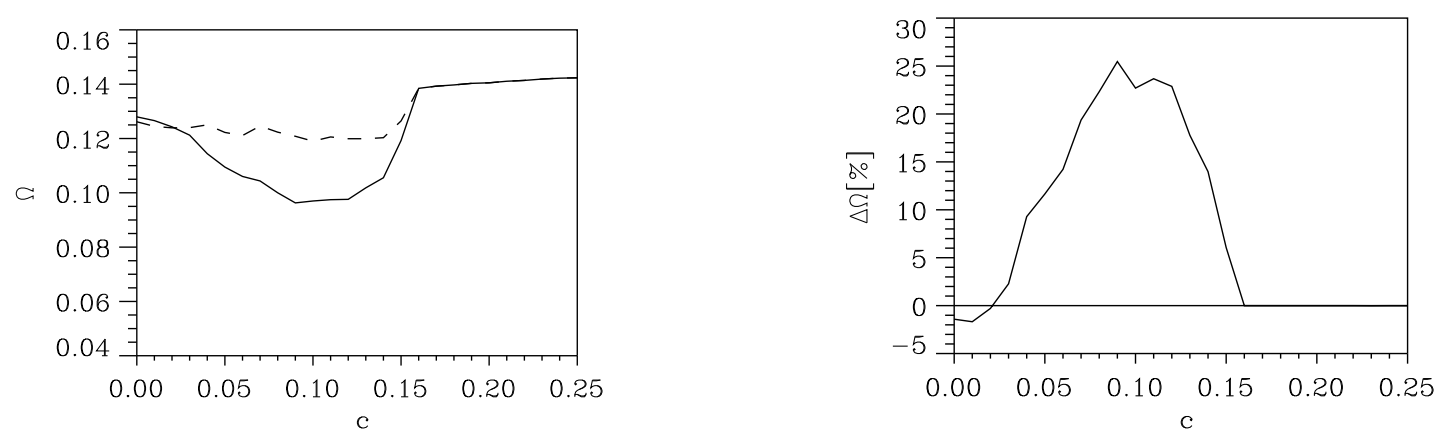

Abbildung 4.28.: Frequenzunterschied $\Delta \Omega$ gegen die Kopplungsstärke $c$ bei bidirektional gekoppelten GL-Gleichungen mit $\beta_{1}=-1.2$ und $\beta_{2}=-0.83$. a) mittlere Frequenz von $u$ (durchgezogene Linie und $v$ (gestrichelte Linie), b) relative Frequenzdifferenz.

Eigenschaft des gekoppelten Gesamtsystems, so daß ein Plateau der Phasendifferenz bei Variation der Kopplungsstärke, welches die Dynamik auch qualitativ verändern kann, als Kriterium für PS prinzipiell ungeeignet ist. Darüberhinaus muß $\widetilde{\Delta \psi}$ innerhalb des phasensynchronisierten Bereiches nicht konstant bleiben, welches die Histogramme in Abb. 4.25 bei einseitiger Kopplung deutlich zeigen. Der Schwerpunkt, welcher $\widehat{\Delta \psi}$ darstellt, verschiebt sich bei steigender Kopplungsstärke von 0 nach $\frac{\pi}{2}$ und ist damit trotz PS weit von einem Plateau entfernt. Das zweite Kriterium für PS, die gleiche Anzahl von Defekten beider Systeme, kann man immerhin als notwendig für PS betrachten. Zum Nachweis von PS müssen die Defekte aber darüberhinaus auch räumlich und zeitlich miteinander synchronisiert sein, so daß mögliche Phasensprünge in den Defekten auch 


\section{Synchronisation und Kontrolle räumlich ausgedehnter Systeme}

zu identischen Sprüngen in den Einzelphasen $\widetilde{\phi}$ führen, welche die Differenz unbeeinflußt lassen. Die Synchronisation von nur der Anzahl der Defekte $N_{d}$ ist damit eher ein schwaches Kriterium, welches die Schwelle zu PS unter Umständen weit unterschätzen kann. Zur Untermauerung dieser Argumente haben wir die Simulationen in [12] wiederholt und die im letzten Abschnitt eingeführten schärferen Kriterien angewandt, welche direkt an die Definition von PS (Glg. (4.16)) bzw. FS (Glg. (4.18)) angelehnt sind. Abbildung 4.28a zeigt die Entwicklung der mittleren Umlauffrequenzen $\Omega$ von $u$ und $v$ in Abhängigkeit von der Kopplungsstärke $c$ und Abb. 4.28b den relativen Frequenzunterschied $\Delta \Omega$ (Glg. (4.17)) beider Systeme in Prozent. Im Bereich $c<0.15$ haben die Systeme teilweise stark verschiedene mittlere Frequenzen, so daß trotz bidirektionaler Kopplung die Dynamik beider Systeme sich auf unterschiedlichen Zeitskalen entwickelt. Für $c \geq 0.16$ verschwindet die Frequenzdifferenz und Frequenzsynchronisation tritt auf. Der relative Phasenunterschied (bei fortgesetzen Phasen $\phi \in \mathbb{R}$ ) beider Systeme nach
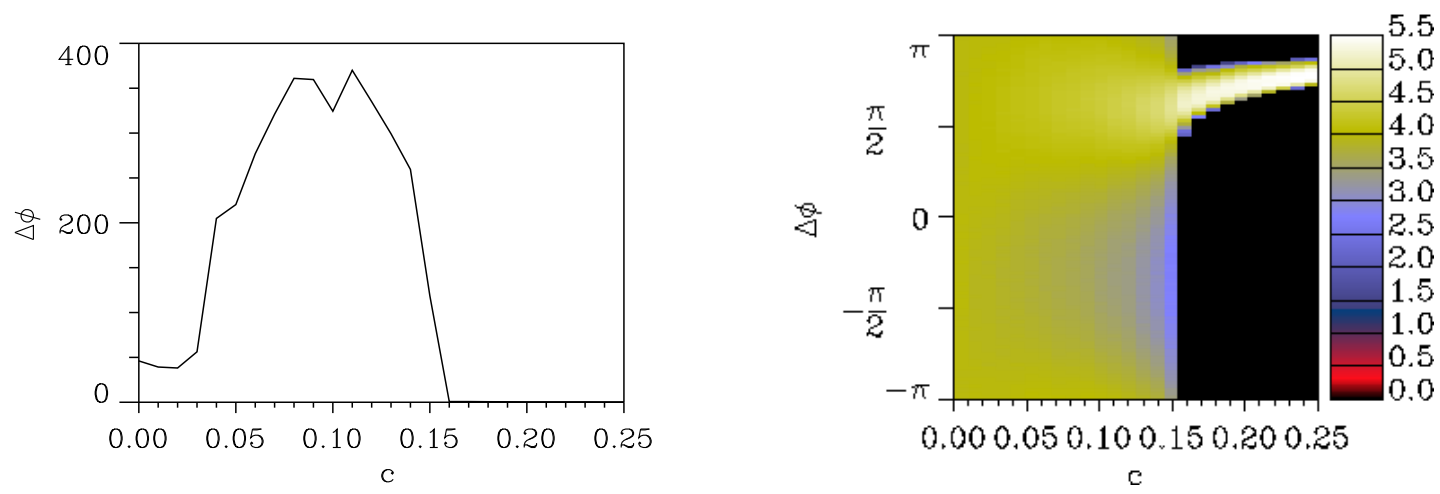

Abbildung 4.29.: a) Relative maximale Phasendifferenz (logarithmische Skala) b) Histogramm der relativen zyklischen Phasendifferenz gegen die Kopplungsstärke $c$ für $\beta_{1}=-1.2$ und $\beta_{2}=-0.83$.

2500 Zeiteinheiten, bei Variation der Kopplung, zeigt Abb. 4.29a. Phasensynchronisation tritt zeitgleich mit FS für $c \geq 0.16$ auf, welches auch durch das Histogramm der relativen (zyklischen) Phasendifferenz $\widetilde{\Delta \phi}$ in Abb. 4.29b bestätigt wird. Damit tritt erst direkt hinter dem in [12] angegebenen Intervall $(0.1 \leq c \leq 0.16)$ Phasensynchronisation auf, welches die dort benutzten Kriterien zum Nachweis von PS disqualifiziert. Der Übergang bei $c \geq 0.16$ zu fast identischer Dynamik kann aber, gemessen durch den raumzeitlichen linearen Korrelationskoeffizienten $\gamma$ in Abb. 4.30, bestätigt werden. Die hohen linearen Korrelationen der raumzeitlichen Muster für $c \geq 0.16$ deuten wieder auf GS $\operatorname{hin}^{13}$. Der größte transversale Lyapunovexponente wird auch für $c \geq 0.16$ negativ (hier nicht gezeigt), so daß vieles für das Auftreten von GS spricht. Frequenzsynchronisation, PS und GS treten somit ab der gleichen Schwelle auf, und da ein funktionaler Zusam-

\footnotetext{
13 Die raumzeitlichen Felder sind sehr ähnlich $u \approx v$, weswegen man diesen Zustand vielleicht als approximative identische Synchronisation bezeichnen könnte. Man darf aber nicht vergessen, daß eine Synchronisationsmannigfaltigkeit $u \equiv v$ nicht existiert, so daß identische Synchronisation streng mathematisch nicht auftreten kann.
} 
4. Synchronisation und Kontrolle räumlich ausgedehnter Systeme

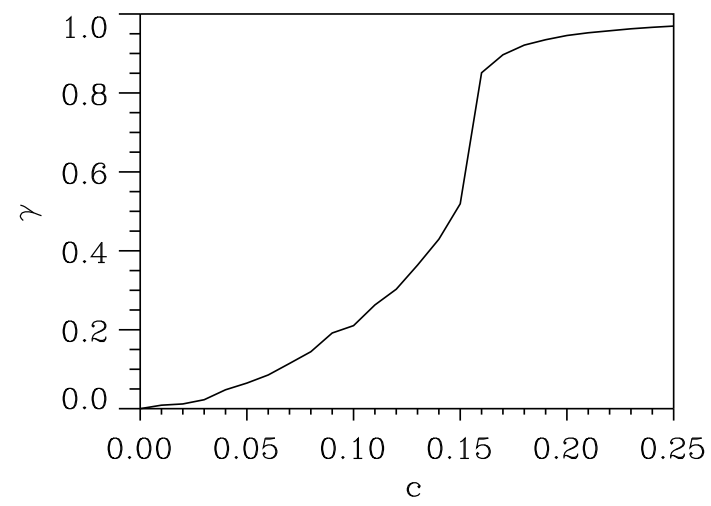

Abbildung 4.30.: Raumzeitlicher linearer Korrelationskoeffizient $\gamma$ gegen die Kopplungsstärke $c$ für $\beta_{1}=-1.2$ und $\beta_{2}=-0.83$ aus Glg. (4.23).

menhang der Flüsse PS und FS impliziert, existiert bei (symmetrischer) bidirektionaler Kopplung und diesen Parameterwerten für dieses System kein eigenständiger phasensynchronisierter Zustand, welcher bei unidirektionaler gekoppelten Systemen über ein weites Kopplungsintervall beobachtet wird. Dies verdeutlicht nochmals, daß uni- und bidirektionale Kopplung nur lokal äquivalent sind [52], während bei globaler fester Kopplungsfunktion sich auch qualitative Unterschiede, sowohl in der Dynamik als auch in den beobachteten (Synchronisations)Phänomenen ergeben können. 


\section{Anwendung der chaotischen Synchronisation in der Systemidentifikation}

Die Synchronisation (chaotischer) Systeme wird, neben dem prinzipiellen wissenschaftlichen Interesse, auch intensiv wegen potentieller Anwendungen erforscht. Die am meisten und vielleicht auch am kontroversesten diskutierte Anwendung liegt in der sicheren Nachrichtenübertragung (siehe z.B. [97, 99]), wo eine Nachricht im breitbandigen chaotischen Trägersignal kodiert wird. Mittels chaotischer Synchronisation zwischen Sender und Empfänger ist es möglich die versteckte Nachricht aus dem Übertragungssignal wieder zu entschlüsseln. Die Sicherheit liegt nun darin begründet, daß ein Angreifer, um die Nachricht zu entschlüsseln, die (vollständige) Systemdynamik, d.h. Zustand, Modell und Parameter, kennen muß, er aber nur das (skalare) Übertragungssignal als Information besitzt. Zumindest für Systeme mit niederdimensionalen Chaos, kann aber die Dynamik gut modelliert werden, so daß die Nachricht oft recht leicht entschlüsselt werden kann [122]. Zur Zeit hofft man, daß hochdimensionale chaotische System die nötige Sicherheit liefern, wobei aber (leider) noch keine Ansätze zu Beweisen von kryptographischer Sicherheit existieren, noch der Rechenaufwand zum Brechen des "Codes“ abgeschätzt werden kann. Aus diesen Gründen ist es zur Zeit noch unklar, ob sichere Nachrichtenübertragung mit chaotischer Synchronisation realisierbar ist. Andererseits kann man chaotische Trägersignale auch zur (unverschlüsselten) Nachrichtenübertragung verwenden [13, 26, 39, 87]. Die Verwendung von chaotischen breitbandigen Trägersignalen kann unter gewissen Bedingungen gegenüber harmonischen Signalträgern durchaus Vorteile bringen, z.B. falls starke Interferenzen auf der Übertragungsstrecke anwesend sind, so daß harmonische Trägersignale stark gestört werden. Auch in digitalen Kommunikationssystemen wird die Anwendung von chaotischen Signalen diskutiert [59, 87], wobei momentan der Trend eher zu nicht synchronisationsbasierenden Systemen hingeht.

In diesem Kapitel, soll auf die Anwendung von Synchronisation in der Systemidentifikation und Parameterbestimmung von dynamischen Systemen näher eingegangen werden. In den Natur- und Ingenieurwissenschaften wird man oft mit dem Problem der Parameterbestimmung von physikalischen Systemen konfrontiert, wobei nur Informationen vom System in Form (skalarer) Zeitreihen vorliegt. Oft sind die Systeme nicht stationär und die Systemparameter ändern sich im Laufe der Zeit, welches z.B. von normalen Verschleißerscheinungen herrühren kann. In technischen Geräten, Kraftwerken, etc. kann dies zum plötzlichen Systemversagen führen, mit möglicherweise katastrophalen Folgen. Durch Parameterbestimmung kann dies oft verhindert werden, indem man zuerst die 


\section{Anwendung der chaotischen Synchronisation in der Systemidentifikation}

kritischen Bereiche des Parameterraumes bestimmt und dann durch regelmässige Bestimmung der Systemparameter frühzeitig ein Versagen erkennt bzw. das System wieder in einen stabilen Bereich führt. Bei linearen Systemen können kleine Parameteränderungen durch Verschiebungen der Spitzen im Fourierspektrum entdeckt und bestimmt werden. Zur Zeit werden aber auch Geräte diskutiert, deren Arbeitsbereich aufgrund hoher Nichtlinearitäten auch komplizierte und/oder chaotische Dynamik zeigen. Beispiele sind stark mischende und elektrochemische Reaktionen, mechanische Lagerschwingungen, etc., wo komplexe Dynamik unvermeidbar oder sogar erwünscht ist und lineare Analysemethoden oft keine zufriedenstellenden Aussagen mehr liefern können.

Bei der vorzustellenden Methode, wird ein gegebenes mathematisches Modell des interessierenden Systems vorrausgesetzt. Falls kein mathematisches Modell des Systems vorliegt, kann mit Methoden aus der nichtlinearen Zeitreihenanalyse [21] ein approximatives Modell der Dynamik bestimmt werden. Dabei wird der vollständige Systemzustand durch Einbettung rekonstruiert, und dann der Fluß im Rekonstruktionsraum durch Polynome, radiale Basisfunktionen, neuronale Netze, etc. als Ganzes approximiert. Damit erhält man dann unabhängig von der Systemstruktur eine gute Approximation der Dynamik im Rekonstruktionsraum, die durch eine diffeomorphe Transformation mit der im Originalzustandsraum verknüpft ist.

Bei vielen realen physikalischen Systemen ist der zugrundeliegende Prozeß aber wohlbekannt und kann in der Form von gewöhnlichen oder partiellen Differentialgleichungen bzw. iterierten Abbildungen modelliert werden. Wenn man nun die Modellgleichungendes Systems kennt, beispielsweise bei elektronischen Schaltkreisen, liegt das Problem darin, die unbekannten Systemparameter für eine vorgegebene Zeitreihe, die meist skalar und diskret abgetastet vorliegt, zu bestimmen. Die Parameterbestimmung kann man als die Lösung eines Minimierungs- oder Fixpunktproblems auffassen, wobei neben den unbekannten Parametervektor noch die nicht gemessen Variablen und die Trajektoriensegmente zwischen den Abtastzeiten als unbekannte Größen auftauchen. Dieser Ansatz wurde bei ODEs mit multiple shooting-Methoden [8] und verwandten Verfahren [15] durchgeführt. Bei räumlich ausgedehnten Systemen wird das Problem aufgrund der hohen Dimension und kontinuierlichen Variablen in Raum und Zeit noch um einiges schwieriger. Die einzige uns bekannte Arbeit zur Systemidentifikation von PDEs ist [136]. Dort wird für das Vektorfeld eine Funktionenklasse angesetzt, deren Parameter durch eine allgemeine Minimierungsprozedur bestimmt werden. Diese Methode ist anwendbar, wenn durch die benutzte Funktionenklasse das wahre Vektorfeld hinreichend gut approximiert werden kann und die gesamte raumzeitliche Systemdynamik mit hinreichend großer Genauigkeit und Auflösung meßbar ist, d.h. etwa in der Größenordnung in der man das System für numerische Integrationen auch diskretisieren würde.

Die Synchronisation von dynamischen (chaotischen) Systemen kann bei diesen Ansätzen zu erheblichen Vereinfachungen in der Komplexität des Problems führen, da hierdurch der vollständige Zustand im Originalzustandsraum aus wenigen Observablen, im Idealfall nur einer einzigen, rekonstruiert werden kannn. Dadurch reduziert sich die Anzahl der zu bestimmenden Größen auf den unbekannten Parametervektor, welches sich bei hyperchaotischen und vor allem räumlich ausgedehnten System als wesentlicher Vorteil herausstellt. Die Anwendbarkeit dieser Methode wurde in [20, 27, 88] anhand iterier- 
ter Abbildungen und niedermensionalen chaotischen kontinuierlichen Systemen demonstriert.

Ein weiterer auf Synchronisation beruhender Ansatz zur Parameterbestimmung ist die Autosynchronisation [86].

Wir wollen hier die Methode aus [88] mit einer verwandten aber schnelleren Variante vergleichen. Neben der Geschwindigkeit untersuchen wir auch die Empfindlichkeit auf additives Rauschen in den Meßsignalen. Bei der Originalmethode [88] wird versucht die gemessene Zeitreihe mit dem Modellsystem zu synchronisieren, wobei nach einer Transientphase der mittlere Synchronisationsfehler der Zeitreihe mit dem Modell bestimmt wird. Dieser Fehler wird dann durch Variation der Systemparameter minimiert. Falls der richtige Parametervektor gefunden ist, tritt IS auf, was gleichbedeutend mit dem Verschwinden des Synchronisationsfehlers ist.

Die Alternative ist statt des gemittelten Synchronisationsfehler, den Fehler zu einem festen Zeitpunkt zu betrachten und für diesen durch Variation der Parameter eine Nullstelle zu finden.

Die Anwendbarkeit der ersten Methode wurde von uns anhand iterierter Abbildungen und ODEs in [88] demonstriert. Beide Strategien werden im folgenden anhand hyperchaotischer Systeme miteinander verglichen. Danach werden noch alle Systemparameter (einschließlich der räumlichen Länge $L$ ) von räumlich ausgedehnten Systemen bestimmt.

\subsection{Synchronisationsbasierende Bestimmung von Systemparametern}

Es wird angestrebt mit einem möglichst allgemeinen Ansatz die Modellparameter von dynamischen Systemen, deren globale Modellierung durch iterierte Abbildungen, gewöhnliche Differentialgleichungen und partielle Differentialgleichugnen vorliegt, zu bestimmen, wobei keinerlei spezielle Eigenschaften der betrachteten Systeme benutzt werden. Z.B. sind die ODEs des Chua-Oszillators in der Lur'e Form, wodurch ein spezieller angepaßter Algorithmus verwendet werden kann [27], womit man sich aber auf eine spezielle Systemklassen einschränkt. Von uns werden keine Voraussetzungen an das spezielle System gestellt. Von realen Systemen gemessene Zeitreihen liegen praktisch immer in abgetasteter Form vor, und dies ist daher bei einem praktisch anwendbaren Algorithmus zu berücksichtigen. Aus diesem Grund wird bei zeitkontinuierlichen Systemen die Methode der zeitdiskreten Kopplung aus Abschn. 3.1.4 zur Synchronisation [96] verwendet, welche trotz zeitdiskreter Daten keinen internen Fehler verursacht.

Damit müssen folgende Voraussetzungen erfüllt werden:

- Das Modell des zu untersuchenden Systems liegt in Form von gewöhnlichen, partiellen Differentialgleichungen bzw. iterierten Abbildungen

$$
\begin{aligned}
\frac{d}{d t} \mathbf{x} & =\mathbf{F}(\mathbf{x} ; \mathbf{p}) \\
\mathbf{x}^{n+1} & =\mathbf{F}\left(\mathbf{x}^{n} ; \mathbf{p}\right),
\end{aligned}
$$

mit $\mathbf{p}$ als unbekanntem Parametervektor des Originalsystems vor. 


\section{Anwendung der chaotischen Synchronisation in der Systemidentifikation}

- Die gemessene Observable des Systems

$$
\mathbf{s}=\mathbf{h}(\mathbf{x})
$$

ist mit einer genügend hohen Abtastrate $T$ abgetastet worden.

- Das dynamische System Glg. (5.1) bzw. Glg. (5.2) läßt sich mit Glg. (5.3) als antreibendem Signal und bekanntem richtigen Parametervektor $\mathbf{p}$ synchronisieren. Dies ist aufgrund der Einbettungstheoreme prinzipiell für skalare Signale immer möglich [126] und zumindest im Computer, aufgrund der großen Anzahl von Kopplungsschemata, fast immer auch realisierbar.

Bis jetzt wurde der Fall der sog. identischen Synchronisation (IS), d.h. p ist bekannt, betrachtet, aber der Parametervektor $\mathbf{p}$ ist ja die zu bestimmende Größe. In diesem Fall, wenn die Parameter des treibenden Systems nicht exakt die gleichen wie die des getriebenen Systems sind, ist IS nicht mehr möglich und der Synchronisationsfehler, der minimiert werden soll, kann eine im Prinzip beliebig komplizierte Funktion der Parameter sein. Der Ausweg ist die verallgemeinerte Synchronisation (GS) aus Abschn. 2.2, die bei entsprechender Kopplung gegen Parameterunterschiede im getriebenen System sehr robust ist.

Der Algorithmus stellt sich nun folgendermaßen dar:

1. Wähle Anfangswerte für den unbekannten Parametervektor $p$

2. Versuche die gemessene Zeitreihe mit dem Computermodell mit Parametervektor $p$ im verallgemeinerten Sinne zu synchronisieren (zum Nachweis von GS siehe Abschn. 2.2.1).

3. Werte eine Kostenfunktion $E(\mathbf{p})$ aus. (Wobei vor der Auswertung eine gewisse Transientphase zum Erreichen von GS abgewartet werden sollte.)

4. Erfüllt die Kostenfunktion eine Abbruchbedingung, dann sind wir fertig, ansonsten

5. variiere den Parametervektor und gehe zu (3).

Damit muß noch eine Kostenfunktion $E(\mathbf{p})$ und eine Strategie zur geschickten Variation der Parameter aufgestellt werden. Als Kostenfunktion bietet sich der Synchronisationsfehler $\mathbf{s}-\mathbf{h}(\mathbf{x})$ an. Dieser hängt implizit vom Parametervektor $\mathbf{p}$ ab und verschwindet, falls die richtigen Parameter von dem der Zeitreihe zugrundeliegendem System vorliegen. Damit stellt diese Größe ein direktes Maß für die Güte des verwendeten Parametersatzes p des Computermodells dar. Wir benutzen in dieser Arbeit folgende Kostenfunktionen:

- Der über ein Zeitintervall gemittelte Synchronisationsfehler

$$
E(\mathbf{p})=\sqrt{\frac{1}{N} \sum_{n=1}^{N}\left[\mathbf{s}^{n}-\mathbf{h}\left(\mathbf{y}^{n}\right)\right]^{2}} .
$$




\section{Anwendung der chaotischen Synchronisation in der Systemidentifikation}

Es gilt $E(\mathbf{p}) \geq 0$, so daß die Suchstrategie Minimierung von $E$ ist. Die Passivität des getriebenen Systems, welche GS sichert, wird während der Parametervariationen laufend überprüft. Falls diese verloren geht, kann man zusätzlich noch den bedingten Lypunovexponenten $\lambda_{\perp}$ in diese integrieren.

Das Auftreten von verallgemeinerter Synchronisation führt oft zu einer glatten Abhängigkeit des Synchronisationsfehlers von den Parametern [88], wodurch man hoffen kann, daß die Kostenfunktion bei den wahren Parameterwerten ein tiefes Minimum besitzt, welches vielleicht sogar in einem weiten Bereich eindeutig ist. Damit muß der Algorithmus nur nach einem lokalen eindeutigen Minimum suchen und man kann deswegen auf einen relativ einfachen Minimierungsalgorithmus zurückgreifen, welcher im Vergleich zu globalen Suchverfahren, wie z.B. simulated annealing, im Hinblick auf die Rechenzeit sicherlich einige Vorteile bringen wird. Als Minimierungsroutine wurde ein Standardverfahren aus [106] verwendet, welches auf dem Powellminimierungsalgorithmus in mehreren Dimensionen beruht.

- Der lokale Synchronisationsfehler

$$
e(\mathbf{p})=s-h(\mathbf{y}) \text {. }
$$

$e$ kann größer und kleiner Null werden und sollte bei den richtigen Parametervektor $p$ identisch Null sein, so daß hier die Suchstrategie im finden der Nullstelle von $e(\mathbf{p})$ besteht. Hierzu wurde ein global konvergentes gedämpftes Newtonverfahren in mehreren Dimensionen aus [106] verwendet, welches die Nullstellen einer $n_{p}=\operatorname{dim}\{\mathbf{p}\}$ dimensionalen Funktion sucht, so daß wir einen $n_{p}$ dimensionalen Fehlervektor zu bilden haben. Für das Nullstellenproblem wird ein Delayvektor von lokalen Synchronisationsfehlern benutzt, der nach Abwarten einer Transientphase von $n_{T}$ Samples gebildet wird. Das heißt, daß bei einer Zeitreihe der Länge $N$ und einem Delay von $d=1$, der $n_{p}$-dimensionale Fehlervektor

$$
e_{i}(\mathbf{p})=s^{n_{T}+i d}-h^{n_{T}+i d}(\mathbf{y}), i=0, \ldots, n_{p}-1
$$

gebildet wird.

Die beiden Verfahren sind in gewisser Weise äquivalent, da im Allgemeinen, jedes Nullstellenproblem auch als Minimierungsproblem umgeschrieben werden kann. Der Vorteil liegt in den schelleren Konvergenzeigenschaften des Newtonverfahrens, wobei sich das Minimierungsverfahren als etwas robuster gegen Modellungenauigkeiten und Rauscheinflüsse herausstellen wird. Die Parametersuche von niederdimensionalen chaotischen Systeme durch Minimierung von Glg. (5.4) wurde von uns numerisch und experimentell in [88] anhand des Chua-Oszillators ausführlich untersucht, weswegen wir uns in dieser Arbeit gleich höherdimensionalen Systemen zuwenden wollen.

\subsection{Hyperchaotische verrauschte Systeme}

Die Parameterbestimmung durch Nullstellenbestimmung von Glg. (5.5) soll am hyperchaotischen verallgemeinerten Rösslersystem demonstriert werden. Das wohlbekannte 

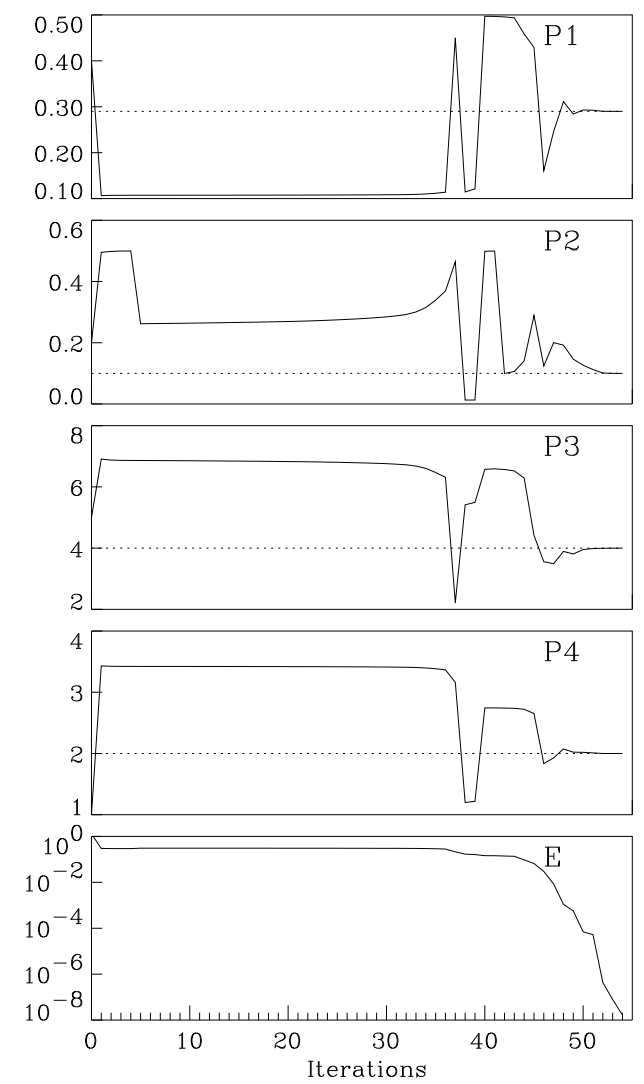

(a) 5-Dim

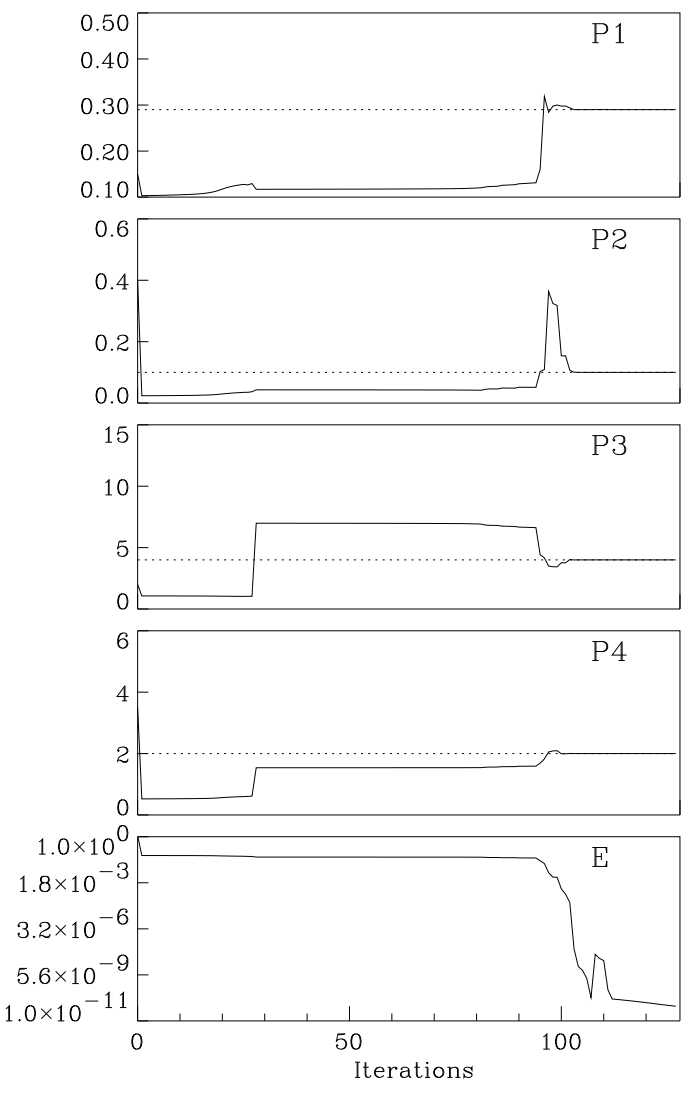

(b) 11-Dim

Abbildung 5.1.: Konvergenz der Parameter beim 5- und 11-dimensionalen hyperchaotischen Rösslersystem. Die Parameter wurden durch Bestimmung der Nullstellen von Glg. (5.5) durchgeführt, wobei auf der Abszisse die Iterationen des Newtonverfahrens aufgetragen sind. Die Zeitreihe wurde mit den Parametern $p_{1}=0.29, p_{2}=0.1, p_{3}=4.0, p_{4}=2.0$ erzeugt, die im Bild gestrichelt eingezeichnet sind.

Rösslersystem kann durch lineare Kopplung mit $M-3$ zusätzlichen Freiheitsgraden zu einem hyperchaotischen System M'ter Ordnung mit hochdimensionaler Dynamik verallgemeinert werden [9, 72]. In [9] wurde gezeigt, daß Glg. (5.7) hyperchaotische Dynamik, mit steigender Anzahl von positiven Lyapunovexponenten bei zunehmenden $M$, zeigt.

$$
\begin{aligned}
\frac{d x_{1}}{d t} & =-x_{2}+p_{1} x_{1} \\
\frac{d x_{m}}{d t} & =x_{m-1}-x_{m+1} \quad(m=2, \ldots, M-1) \\
\frac{d x_{M}}{d t} & =p_{2}+p_{3} x_{M}\left(x_{M-1}-p_{4}\right)
\end{aligned}
$$




\section{Anwendung der chaotischen Synchronisation in der Systemidentifikation}

Es wurden alle 4 Parameter von Glg. (5.7) für den Fall $M=5$ und $M=11$ mit dem Nullstellenverfahren bestimmt. Dazu wurde einer Zeitreihe der $x_{1}$-Komponente der Länge 500, mit einer Abtastrate von 1 für das 5- und 11-dimensionale System mit den Parametern $p_{1}=0.29, p_{2}=0.1, p_{3}=4.0, p_{4}=2.0$, numerisch erzeugt. Bestimmung der Lyapunovdimension ergibt für das 5-dimensionale System einen Wert von $D_{L} \approx 4.029$ und für $M=11$ von $D_{L} \approx 10.029$. Die Synchronisation wurde durch periodische Ersetzung („sporadic driving“) der $x_{1}$-Komponente in den Modellgleichungen erreicht. Für den Fehlervektor im Nullstellenverfahren wurde ein Delay von 1 verwendet. Die Ergebnisse eines typischen Suchlaufes des Verfahrens sind in Abb. 5.1 aufgetragen. Aufgrund der schlechten Schätzung der Parameterstartwerte $\mathbf{p}_{0}$ konvergiert das Verfahren am Anfang sehr langsam, aber in der Nähe der richtigen Werte adaptiert es sehr schnell und der Synchronisationsfehler fällt auf die verwendete Rechengenauigkeit ab. Die Anzahl der benötigten Iterationen hängt stark von den Parameterstartwerten ab. Verschiedene Läufe haben gezeigt, daß typischerweise 50 - 150 Iterationen nötig sind, wobei beim höherdimensionalen System im Mittel etwa die doppelte Anzahl benötigt wurde. Die richtigen Parameter werden für die meisten Parameterstartwerte gefunden, so daß auch hier die Fehlerlandschaft relativ gutmütig und glatt sein wird.

In Abb. 5.2 ist der Einfluß der zur Verfügung stehenden Länge der Zeitreihe auf die Parameterbestimmung untersucht. Es wurde auch hier ein Delay von 1 für den Fehlervektor verwendet und Parameteranfangsbedingungen, die ähnlich wie in Abb. 5.1, weit entfernt von den richtigen liegen, verwendet. Gezeigt sind die gefundenen Parameterwerte einzelner Suchläufe, wobei die Abweichungen von den echten Werte repräsentativ für alle Läufe bei dieser Länge sind. Beim 5-dimensionalen System muß die Zeitreihe eine minimale Länge von $\approx 40^{1}$ besitzen, damit mit hinreichender Genauigkeit die Parameter bestimmt werden können, während für das 11-dimensionale System schon eine Länge von $\approx 180$ benötigt wird. Es fällt auf, daß einige Parameter empfindlicher auf kurze Längen reagieren als andere, so daß der Synchronisationsfehler nicht mehr auf Rechengenauigkeit abfällt. Dies deutet darauf hin, daß die Parameterbestimmung noch in der Transientphase stattgefunden hat, so daß die Systeme bei Berechnung der Fehlerfunktion noch nicht vollständig synchronisiert waren. Daß für das höherdimensionale System längere Zeitreihen erforderlich sind, ist nicht verwunderlich, da die Kopplung in der $x_{1}$-Komponente endliche Zeit braucht um die $x_{M}$-Koponente beeinflussen zu können und dadurch die Zeit zum synchronisieren sicherlich ansteigen wird.

Nachdem die Anwendbarkeit der Nullstellenstrategie zur Parameterbestimmung gezeigt wurde, soll diese nun mit dem Minimierungsverfahren verglichen werden, wobei insbesondere auf die Empfindlichkeit gegenüber additivem Rauschen geachtet werden soll. Dazu wurde wieder das 5-dimensionale verallgemeinerte Rösslersystem mit den gleichen Parametern verwendet und auf die "gemessene“ Zeitreihe weißes Rauschen mit vorgegebenem Signalrauschverhältnis (SNR) addiert, so daß nun ein verrauschtes treibendes Signal der Form $s=\hat{x}_{1}+\eta$ vorliegt. Für das Minimierungsverfahren wurde nach einer Einschwinglänge von 500 Samples der Fehler über eine Zeitspanne von 300 Samples

${ }^{1}$ Die Anzahl der Samples entspricht aufgrund der Abtastrate von 1 auch gleichzeitig den Zeiteinheiten des Systems. 
5. Anwendung der chaotischen Synchronisation in der Systemidentifikation
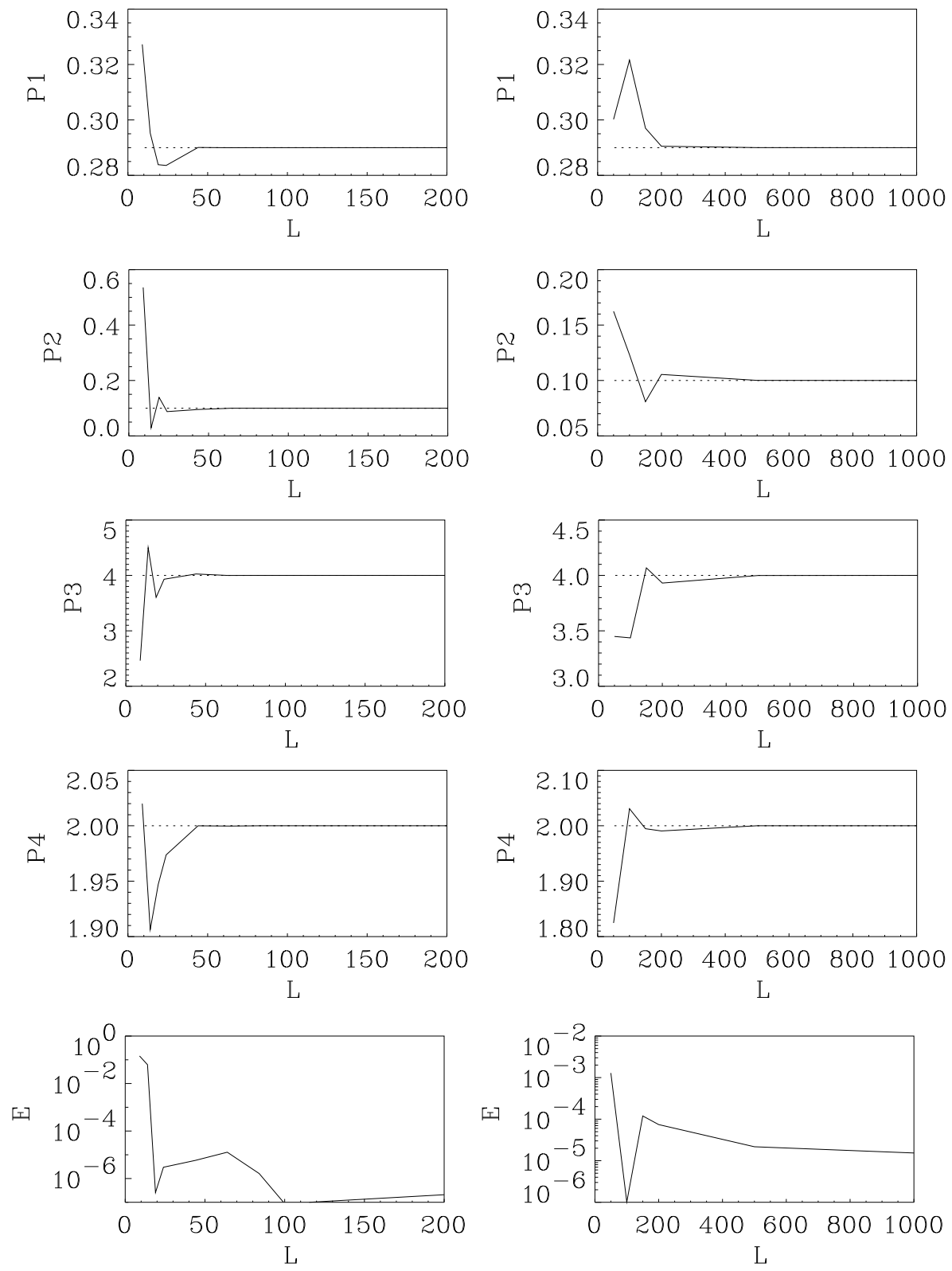

a) 5-dim

b) 11-dim

Abbildung 5.2.: Konvergenz der Parameter und des Fehlers gegen die Länge der Zeitreihe beim a) 5-dimensionalen und b) 11-dimensionalen Rösslersystem. Die Zeitreihe wurde mit den Parametern $p_{1}=0.29, p_{2}=0.1, p_{3}=4.0, p_{4}=$ 2.0 erzeugt, welche im Bild durch die gestrichelten Linien angedeutet sind. 
5. Anwendung der chaotischen Synchronisation in der Systemidentifikation
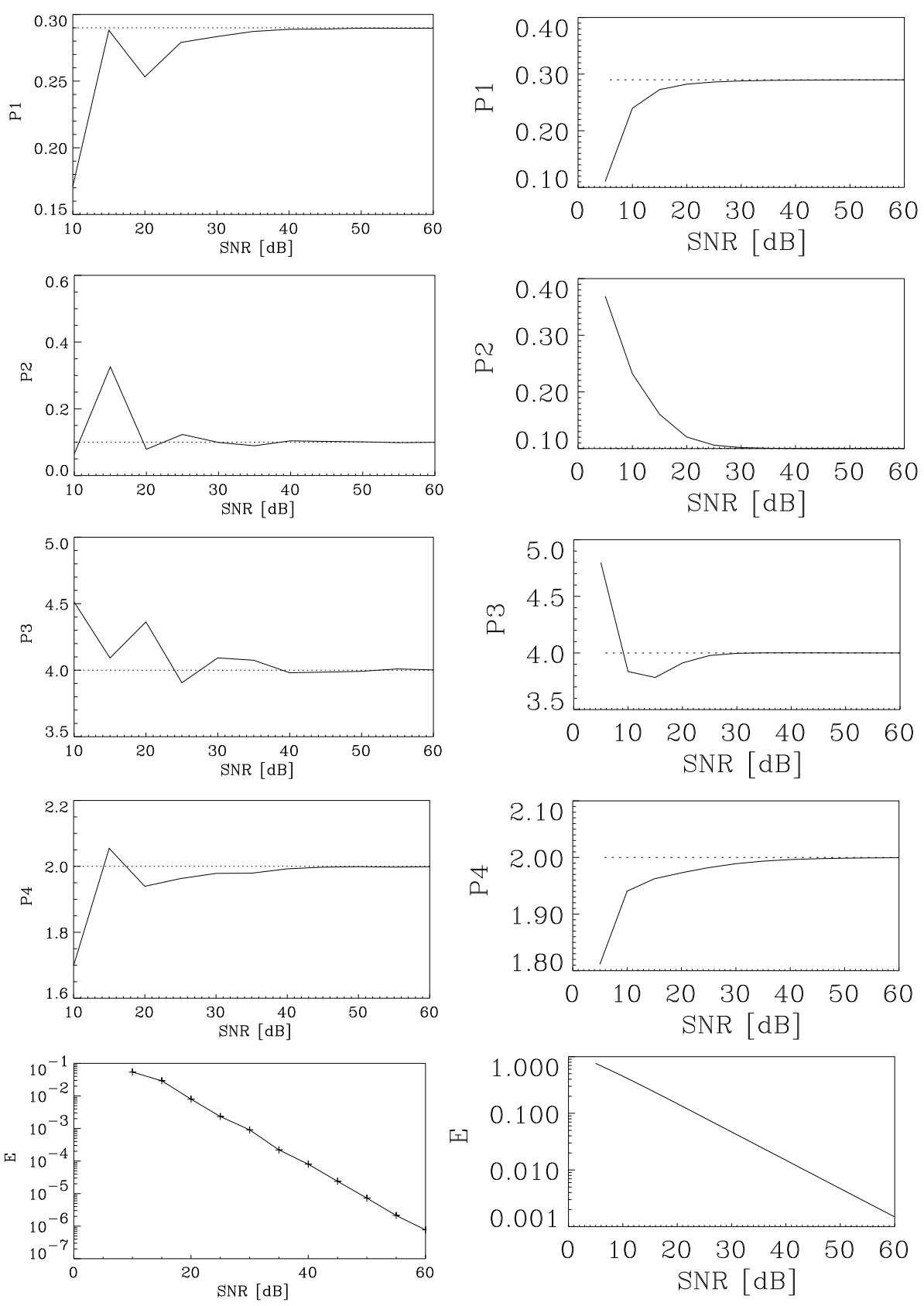

a) Nullstellenverfahren

b) Minimierungsverfahren

Abbildung 5.3.: Konvergenz der Parameter und des Fehlers gegen SNR beim a) Nullstellenverfahren und b) Minimierungsverfahren, mit den Parametern $p_{1}=0.29, p_{2}=0.1, p_{3}=4.0, p_{4}=2.0$. 


\section{Anwendung der chaotischen Synchronisation in der Systemidentifikation}

gemittelt. Beim Nullstellenverfahren wurde eine Zeitreihe ungefähr gleicher Länge verwendet, wobei die ersten 700 Samples für die Transiente verwendet wurden und der Fehlervektor für die 4 Parameter einen Delay von 20 Samples hatte. Die Kostenfunktion für das Nullstellenverfahren wurde aber, aufgrund des nun vorhandenen Rauschens, leicht modifiziert. Es wurde, ähnlich wie beim Minimierungsverfahren, eine Zeitmittelung über das Delayintervall eingeführt, welche die Kostenfunktion etwas unempfindlicher gegenüber Rauschen machen soll, ohne die guten Konvergenzeigenschaften zu vermindern

$$
e=\max \left(\begin{array}{l}
\sum_{i=1}^{n_{\text {delay }}}\left(s^{i}-x_{1}^{i}\right)>0 \\
\sum_{i=1}^{n_{\text {delay }}}\left(s^{i}-x_{1}^{i}\right)<0
\end{array}\right)
$$

Dabei heißt max, daß die betragsmäßig größte Summe als Fehler genommen wird. In Abb. 5.3 sind die beiden Verfahren bei verschiedenen SNRs gegenübergestellt. Beide Methoden finden die richtigen Parameterwerte bis hinunter zu Signalrauschverhältnissen von $\approx 15$, was je nach Anwendung gut oder schlecht sein kann. Man darf aber nicht vergessen, daß nur ein Signal gemessen wurde und die Zeitreihe für globale Modellierungsanwendungen sehr kurz ist. Hat man längere Zeitreihen zur Verfügung, kann man versuchen durch nichtlineare Rauschunterdrückung das Signal zu verbessern, oder man benutzt längere Mittelungsintervalle bzw. Delayzeiten zur Berechnung der Kostenfunktion, welche einiges von den Rauscheinflüssen herausmitteln und dadurch die Methoden gegenüber Rauschen robuster machten. Den Einfluß des Rauschens auf die Qualität der Synchronisation sieht man am deutlichsten im Plot des gemittelten Synchronisationsfehlers (Kostenfunktion) gegen das SNR, wo der Synchronisationsfehler offensichtlich nur von der Stärke des Rauschens bestimmt wird. In [35] haben wir den Einfluß von Rauschen auf die Parameterbestimmung neben dem hyperchaotischen Rösslersystem noch bei mehreren niederdimensionalen Systemen getestet, wo im wesentlichen das gleiche Verhalten beobachtet werden konnte. Eine Verbesserungsmöglichkeit besteht möglicherweise noch in der Verwendung anderer Kopplungsvarianten, bei denen das System weniger stark auf Rauschen reagiert, wodurch die Robustheit des Verfahrens noch verbessert werden könnte.

\subsection{Räumlich ausgedehnte Systeme}

Als zweite Systemklasse für hochdimensionales Chaos sollen die Systemparameter von PDEs bestimmt werden. Zur Synchronisation wird die Sensorkopplung aus Abschn. 4.3 verwendet, so daß wir nun $N$ skalare Zeitreihen vorliegen haben. Zusätzlich gehen wir davon aus, daß die gemessenen Zeitreihen nicht nur räumlich, sondern auch zeitlich diskret abgetastet vorliegen, so daß die zeitkontinuierliche Sensorkopplung in Glg. (4.9) noch auf diskrete Zeitreihen verallgemeinert werden muß. Dazu verwenden wir wieder das Konzept des „sporadic driving“, diesmal aber in Zeit und Raum. Wir haben $N$ Zeitreihen, die in Zeitabständen $t_{s}$ abgetastet wurde. Die Kopplung besteht nun darin, daß zu den Abtastzeiten $n t_{s}$ die entsprechende Variable des Computermodells in Richtung des synchronisierten Zustandes an den Kopplungsintervallen durch

$$
v_{k}^{t}=v_{k}^{t}+\epsilon\left(\bar{u}_{k}^{t}-\bar{v}_{k}^{t}\right), \quad t=n t_{s}
$$




\section{Anwendung der chaotischen Synchronisation in der Systemidentifikation}

geführt wird. Zwischen den Kopplungsintervallen entwickelt sich das System frei in Raum und Zeit nach Glg. (4.5). Aus experimenteller Sicht stellt dies vielleicht eine etwas unrealistische Kopplungsart dar, aber da wir mit einem Computermodell synchronisieren, haben wir alle Freiheiten. Die Synchronisation ist damit auch mit nur einer einzigen skalaren Zeitreihe möglich, wenn man die $N$ Sensoren zeitlich nacheinander abtastet, wobei die Abtastzeit für jeden einzelnen Sensor konstant bleibt, aber die Gesamtabtastrate sich um den Faktor $N$ erhöht. Man hat dann eine Zeitreihe, wo jede $\frac{t_{s}}{N}$ Sek. ein Sample von dem nächsten Sensor gemessen wird, so daß man alle $t_{s}$ Sek. ein Sample von dem gleichen Raumbereich erhält. Diese Variante wird der Einfachheit halber hier nicht betrachtet.

\subsubsection{Kuramoto-Sivashinski Gleichung}

Mit der Kopplung aus Glg. (5.9) sollen nun die Systemparameter einer Kuramoto-Sivashinski Gleichung [24, 49] der Form

$$
\begin{aligned}
& \frac{\partial u}{\partial t}+\frac{\partial^{4} u}{\partial x^{4}}+p_{1}\left(\Delta u+\frac{1}{2}\left(\frac{\partial u}{\partial x}\right)^{2}\right]+p_{2} \frac{d w}{d t}=0 \\
& \frac{d w}{d t}=-\frac{p_{2}}{4 \pi} \int_{0}^{2 \pi}\left(\frac{\partial u}{\partial x}\right)^{2} d x, \quad x \in\left[0, p_{3}=L\right]
\end{aligned}
$$

mit periodischen Randbedingungen bestimmt werden ${ }^{2}$. Der Term in Glg. (5.11) dient zur Entfernung der Mittelwertdynamik und geht aus einer zeitabhängigen Galileitransformation hervor. Zur Parameterbestimmung wird das Nullstellenverfahren benutzt, wobei jetzt als Kostenfunktion

$$
e=\max \left(\begin{array}{c}
\sum_{k=1}^{N}\left(\bar{s}_{k}-\bar{v}_{k}\right)>0 \\
\left|\sum_{k=1}^{N}\left(\hat{s}_{k}-\hat{v}_{k}\right)<0\right|
\end{array}\right)
$$

angesetzt wird, die zu den Abtastzeiten $n t_{s}$ ausgewertet wird. Der obere Ausdruck ist als Summation über die Sensoren, deren lokaler Synchronisationsfehler $\left(\bar{s}_{k}-\bar{v}_{k}\right)$ größer bzw. kleiner als Null ist, zu verstehen, wobei für $e$ das Maximum der Summen verwendet wird. Die Kostenfunktion erscheint sehr kompliziert, hat aber den Vorteil, daß alle Sensoren im gesamten Raum in einer skalaren Fehlerfunktion zusammengefaßt werden können und sich die einzelnen lokalen Synchronisationsfehler nicht wegmitteln können, welches Vorteile in der Konditionierung des Problems mit sich bringt. Gleichung 5.10 zeigt für $p_{1}=200, p_{2}=1.0, p_{3}=L=2 \pi$ raumzeitliches Chaos. Zur Parametersuche wurde Glg. (5.10) durch Diskretisierung der partiellen Ableitungen im Raum, in ein System von 100 gekoppelten gewöhnlichen Differentialgleichungen umgewandelt, welches dann durch einen Standard ODE-Löser mit adaptiver Schrittweitensteuerung integriert wurde.

\footnotetext{
${ }^{2}$ Die Kuramoto-Sivashinski Gleichung (5.10) zeigt qualitativ ähnliche Dynamik, wie die im Abschnitt über die Sensorkopplung. Wir verwenden hier diese Variante, weil hier neben der Länge noch zwei weitere freie Parameter auftauchen.
} 
5. Anwendung der chaotischen Synchronisation in der Systemidentifikation

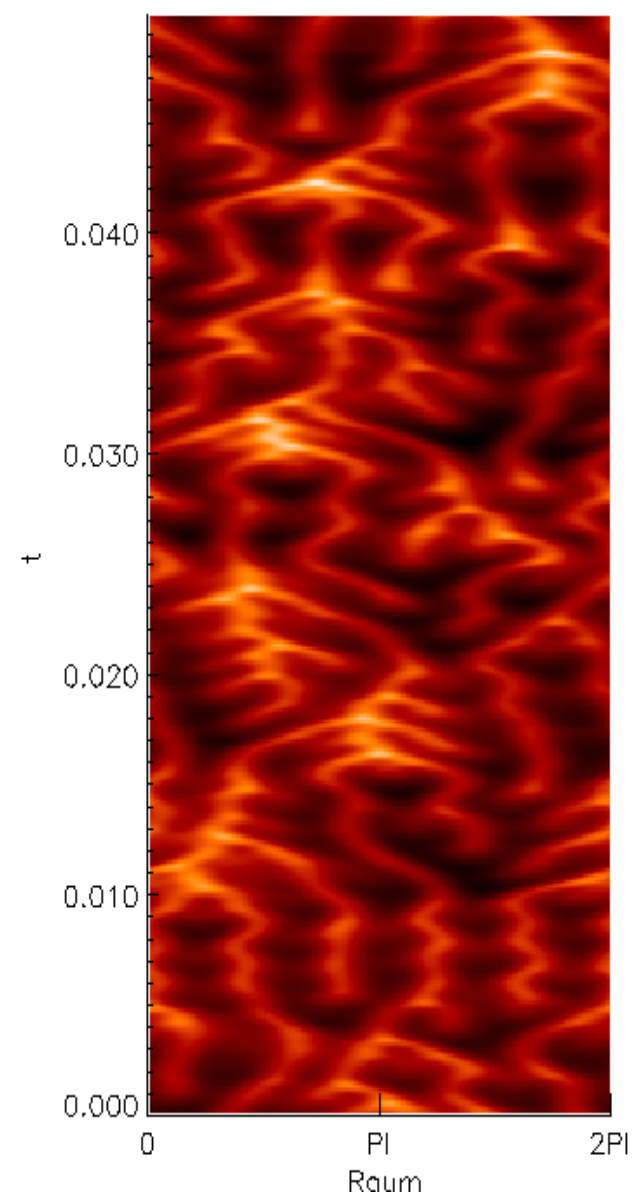

(a) Dynamik
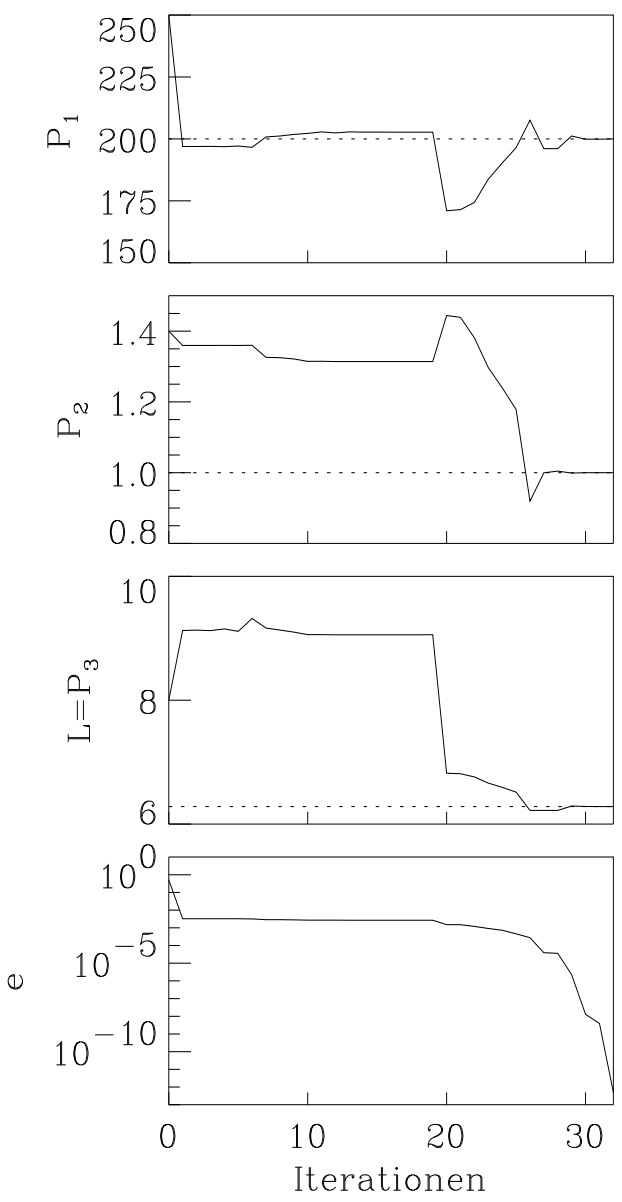

(b) Konvergenz der Parameter

Abbildung 5.4.: Dynamik und Konvergenz der Parameter bei der Kuramoto-Sivashinki Gleichung. $p_{1}=200, p_{2}=1.0, p_{3}=L=2 \pi$

Es wurden dann, mit den Parametern $p_{1}=200, p_{2}=1.0, p_{3}=L=2 \pi$ an $N=9$ äquidistanten Sensoren mit einer Breite von $L_{I}=\frac{2 \pi}{13}$, die Zeitreihen mit einer Abtastzeit von $t_{s}=10^{-4}$ generiert. Dies entspricht einen räumlichen Abstand der Sensoren von $d_{\text {kopp }}=\frac{2 \pi}{10}$, wobei die kleine Abtastzeit $t_{s}$ aus der schnellen Dynamik von Glg. (5.10) bei diesen Parameterwerten resultiert, aber keineswegs als quasikontinierlich angesehen werden kann. Abbildung 5.4a zeigt die chaotische Dynamik für diese Parameterwerte. Für die Parametersuche wurden $N=9$ Zeitreihen der Länge 310 Samples benutzt, wobei der Fehlervektor mit einem Delay von 1 erzeugt wird. Die Systeme wurden dann mit der Kopplung aus Glg. (5.9) und $\epsilon=1.0$ synchronisiert. Die Konvergenz der Parameter zeigt Abb. 5.4b. Die Systemparameter, wo die räumliche Ausdehnung $p_{3}=L$ mit enthalten ist, konvergieren sehr schnell und zielstrebig auf die exakten Werte. 


\subsubsection{Ginzburg-Landau Gleichung}

Als weiteres Beispiel soll die 1-dimensionale komplexe Ginzburg-Landau Gleichung aus Absch. 4.3.2

$$
\frac{\partial u}{\partial t}=p_{1} u-\left(1-i p_{2}\right)|u|^{2} u+\left(1+i p_{3}\right) \Delta u, \quad x \in\left[0, p_{4}=L\right],
$$

mit periodischen Randbedingungen dienen. Die Gleichung wurde, wie in Abschn. 4.3.2.1, mit dem Crank-Nichelson-Schema gelöst. Zur Synchronisation wurde, wie bei der Kuramoto-Sivashinski Gleichung, raumzeitliches „sporadic-driving“ (Glg. (5.9)) benutzt. Als Kostenfunktion für das Nullstellenverfahren wurde Glg. (5.12) verwendet, wobei aufgrund des komplexen Feldes $\|\bar{u}\|$ verwendet wurde.

Als erstes wurde die Ginzburg-Landau Gleichung im phasenturbulenten Regime, für die Parameter $p_{1}=1.0, p_{2}=2.0, p_{3}=0.7, p_{4}=L=40 \pi$, mit einem räumlichen Gitter von 1000 Gitterpunkten gelöst, wobei an $N=99$ äquidistanten Punkten Sensorzeitreihen mit eine Abtastzeit von $t_{s}=0.4$ abgenommen wurden. Die Sensoren hatten einen Abstand von $\frac{p_{4}}{100}$ und eine Breite von $l=0.5$. In Abb. 5.5a sieht man die Konvergenz der 4 Parameter für ein Delay des Fehlervektors von 1 und eine Zeitreihelänge von 500 Samples. Für die defektturbulente Dynamik des zweiten Parametersatzes $p_{1}=1.0, p_{2}=2.0, p_{3}=1.2, p_{4}=L=40 \pi$ wurde eine Zeitreihe der Länge 300 Samples, bestehend aus $N=33\left(d=\frac{p_{4}}{100}\right)$ äquidistanten Sensoren der Breite $l=3.5$ mit einer Abtastzeit von $t_{s}=0.4$, erzeugt. Die Konvergenz der Parameter zeigt Abb. 5.5b. Alle Systemparameter inklusive der räumlichen Ausdehnung $p_{4}=L$ konnten damit in beiden dynamischen Regimen $\left(D_{L} \approx 13\right.$ für Phasenturbulenz und $D_{L} \approx 40$ für Defektturbulenz) korrekt bestimmt werden. Dies zeigt, daß auf Synchronisation beruhende Parametersuchstrategien auch bei räumlich ausgedehnten Systemen erfolgreich angewendet werden können, wobei der Rechenaufwand (ca. eine Stunde auf einer Standardworkstation) vertretbar ist. Man beachte, daß im phasenturbulenten Regime sehr schmale Sensoren verwendet wurden, wodurch weitaus mehr Kopplungssignale als bei Defektturbulenz notwendig waren, wo relativ breite Sensoren verwendet wurden. Dies zeigt einmal mehr den Vorteil der Verwendung von räumlich ausgedehnten Sensoren/Aktuatoren zur Kopplung. Die Ergebnisse bleiben stabil bei kleinen additiven Rauschen auf die Sen-

sorsignale. Es ist anzunehmen, daß die Parameterbestimmung ähnlich robust gegenüber Rauschen ist, wie das hyperchaotische Rösslersystem, was aber nicht systematisch untersucht wurde. 
5. Anwendung der chaotischen Synchronisation in der Systemidentifikation
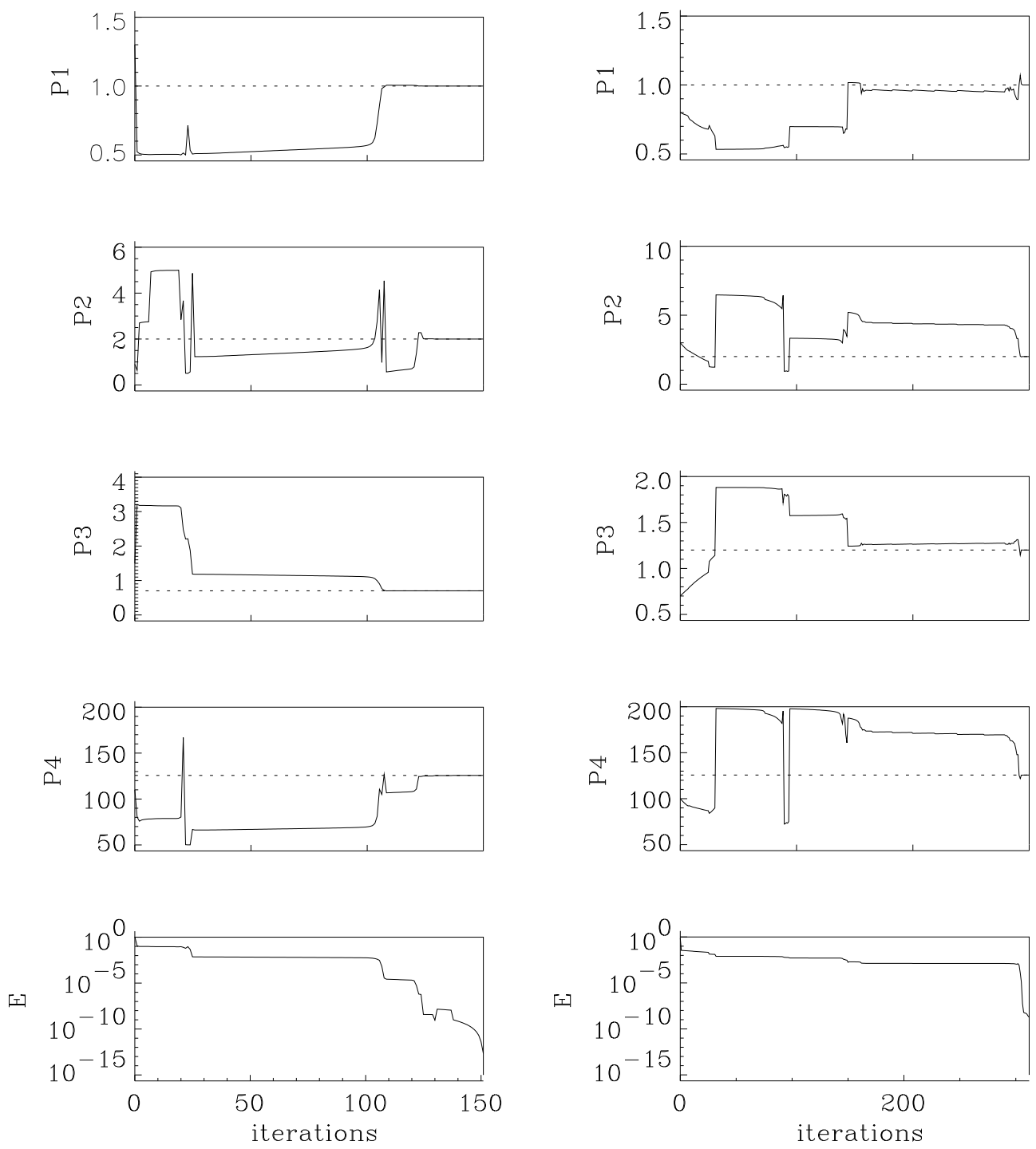

(a) Phasenturbulenz

(b) Defektturbulenz

Abbildung 5.5.: Parameterbestimmung der Ginzburg-Landau Gleichung. Konvergenz der Parameterschätzwerte während der Nullstellenbestimmung, die gestrichelte Linie zeigt die exakten Werte. Die jeweiligen Kopplungsparameter sind im Text erklärt. 


\section{Zusammenfassung und Ausblick}

Die Synchronisation chaotischer Systeme hat sich im letzten Jahrzehnt zu einem breitgefächerten und vielstudierten Gebiet innerhalb der nichtlinearen Dynamik entwickelt. Dabei haben sich im wesentlichen drei qualitativ unterschiedliche Synchronisationsformen, identische, verallgemeinerte und Phasensynchronisation, manifestiert, die alle auch experimentell beobachtet werden. Die beiden ersten Varianten, welche eine funktionale Beziehung zwischen den Flüssen fordern, können innerhalb einer gemeinsamen Theorie behandelt werden, wobei die Phasensynchronisation, die nur partielle synchrone Eigenschaften der gekoppelten Systeme fordert, noch nicht in eine universelle Beschreibung integriert werden konnte. Es sind aber bei weitem noch nicht alle Fragestellungen geklärt, so haben z.B. bei der verallgemeinerten Synchronisation Phänomene, wie subharmonische Einrastung (siehe Abschn. 2.2.2), letztlich zu einer erweiterten Definition für verallgemeinerte Synchronisation geführt. Die Existenz von fraktalen Abbildungen zwischen den Flüssen hat eine tiefergehende Untersuchung von verallgemeinerter Synchronisation im Rahmen der Theorie invarianter Mannigfaltigkeit angestoßen (siehe Abschn. 2.2.3) und die Notwendigkeit von mathematisch exakten Resultaten zum Nachweis und zu den Eigenschaften von verallgemeinerter Synchronisation unterstrichen. In Abschn. 3.1.5 wurde die Verwandschaft der (chaotischen) Synchronisation zur Konstruktion eines nichtlinearen Beobachters aus der Kontrolltheorie diskutiert. Inzwischen besteht ein reger Kontakt zwischen Wissenschaftlern aus beiden Gebieten, der sich mit Sicherheit in Zukunft als gewinnbringend für beide Seiten erweisen wird.

Bei der identischen Synchronisation besteht weiterhin Bedarf an Kopplungsstrategien, die den Informationsfluß zwischen den gekoppelten Systemen minimieren. Ein Ansatz ist die zeitdiskrete Kopplung von kontinuierlichen Systemen („sporadic driving“) in Abschn. 3.1.4). Weiterhin hat bisher kein Kopplungsschema direkt die Eigendynamik des getriebenen Systems ausgenutzt. Aus diesem Grund haben wir in Abschn. 3.2 die dynamische Kopplung eingeführt, wo eine, durch das dynamische System definierte, lokale Kopplung abgeleitet wurde, welche asymptotische Stabilität in jedem Punkt des synchronen Zustandes garantiert.

Neben den niederdimensionalen Systemen kann Synchronisation chaotischer Systeme auch in hochdimensionalen und räumlich ausgedehnten Systemen beobachtet werden. Zur Synchronisation von Systemen, beschrieben durch partielle Differentialgleichungen, haben wir in Abschn 4.3 die Sensorkopplung eingeführt, die auf räumlich ausgedehnten Meßapparaten bzw. Aktuatoren beruht und deswegen auch im Experiment direkt anwendbar sein sollte. Die Sensorkopplung stellt sich gegenüber anderen existierenden Ansätzen zur Kopplung ausgedehnter Systeme in vielen Punkten als überlegen heraus, 


\section{Zusammenfassung und Ausblick}

was z.B. am minimal benötigten Informationsfluß (gemessen an den Kopplungssignalen) deutlich wird. Die Effektivität der Sensorkopplung wurde in Abschn. 5 anhand der Anwendung in der Parameter- und Zustandsrekonstruktion von partiellen Differentialgleichungen demonstriert. Die Parameterbestimmung von Modellgleichungen mittels Synchronisation stellt sich auch als sehr robust gegenüber additivem Rauschen heraus, was anhand einer hyperchaotischen Differentialgleichung untersucht wurde.

Die Synchronisation niederdimensionaler Systeme ist inzwischen recht gut verstanden, was mit ein Grund für das stark gestiegene Interesse an den Synchronisationseigenschaften von hochdimensionalen und räumlich ausgedehnten Systemen darstellt. In Abschn. 4.4 konnten wir (unseres Wissens) als Erste Phasen- und Frequenzsynchronisation zwischen Systemen, beschrieben durch partielle Differentialgleichungen, direkt nachweisen. Weiterhin haben wir starke Indizien dafür, daß bei starker Kopplung verallgemeinerte Synchronisation auftritt.

Die (chaotische) Synchronisation hat in den letzten Jahrzehnten einen regelrechten Boom erfahren, was in Abb. 1.1 anhand der jährlichen Anzahl der referierten Veröffentlichungen eindrucksvoll bestätigt wird. Die Kurve in Abb. 1.1 scheint aber langsam in die Sättigung zu geraten, so daß man sich fragt, ob nach dem Boom nun ein starker Einbruch erfolgen wird. Meiner Einschätzung nach wird dies nicht passieren, da die Synchronisation chaotischer Systeme inzwischen auf breiter Ebene in den Naturwissenschaften und auch der Technik Anwendungen gefunden hat und sich dadurch auch langfristig etablieren kann. Die Zeit der „Goldgräberstimmung“, wo aufgrund der „relativ simplen“ niederdimensionalen Systeme, grundlegende Zusammenhänge, Phänomene, etc. einfacher entdeckt, studiert und erklärt werden konnten, ist wohl vorbei. Der Hauptteil der „übriggebliebenden" offenen Probleme liegt bei äußerst komplexen hochdimensionalen Systemen, wie Felder gekoppelter Oszillatoren zur Modellierung von Neuronenverbänden, Ökosystemen, um nur einige zu nennen. Die Herausforderung liegt heute in dem Transfer des inzwischen fundierten Wissens über die Eigenschaften einfacher gekoppelter Systeme auf solch riesige Komplexe, die sicherlich nicht als Ganzes zu verstehen sein werden. Man wird deswegen eher untersuchen, welche Aufgaben ein Subkomplex erfüllt und wie er mit anderen zusammenarbeitet und kommuniziert. Neben den potentiellen technischen Anwendungen wird meiner Meinung nach die Zukunft der (chaotischen) Synchronisation in allen ihren Formen ihr Hauptpotential in dem Verständnis von solchen Komplexen entwickeln und dort auch einen festen Platz einnehmen. 


\section{A. Ableitung der lokal dynamischen Kopplung bei ODEs}

Das Ziel dieses Abschnittes ist die instabilen Richtungen und Kontraktionsraten aus der linearisierten Flußabbildung

$$
\Phi^{\Delta t}: \quad \mathbf{x}(t) \mapsto \mathbf{x}(t+\Delta t)=(\underline{\underline{\mathbf{I}}}+\Delta t \underline{\underline{J}}) \mathbf{x}=\underline{\underline{\mathbf{D} \Phi}}^{\Delta t} \mathbf{x} ; \quad \underline{\underline{J}}=\left.\frac{\partial \mathbf{f}(\mathbf{x})}{\partial \mathbf{x}}\right|_{\mathbf{x}=\mathbf{x}(t)}
$$

unabhängig von $\Delta t$ zu bestimmen, so daß die dynamische Kopplungsfunktion bei ODEs aus Glg. (3.50) exakt die lineare Instabilität im Geschwindigkeitsfeld $\dot{\mathbf{y}}=\mathbf{f}(\mathbf{x})$ unterdrücken kann. Weiterhin wird gezeigt, daß die expandierenden Richtungen mit Expansionsfaktor direkt aus der Jacobimatrix des Vektorfeldes $\underline{\underline{J}}=\frac{\partial \mathbf{f}(\mathbf{x})}{\partial \mathbf{x}} \mid \mathbf{x}=\mathbf{x}(t)$ bestimmt werden können, so daß der Fluß nicht mehr durch $\underline{\mathbf{D} \Phi}^{\Delta t}$ approximiert werden muß ${ }^{1}$.

Die Singulärwertzerlegung $\underline{\underline{\mathbf{D}} \Phi^{\Delta t}}=\underline{\mathbf{U W V}^{t r}}$, welche wir zur Bestimmung der expandierenden Richtungen der dynamischen Kopplung verwenden, ist eng verwandt mit der Polarzerlegung

$$
\underline{\underline{\mathbf{D}}}^{\Delta t}=\underline{\underline{\mathbf{Q P}}} \text { mit } \underline{\underline{\mathbf{P}}}=\left(\left(\underline{\underline{\mathbf{D}}}^{\Delta t}\right)^{t r} \underline{\underline{\mathbf{D}}}^{\Delta t}\right)^{\frac{1}{2}}
$$

wobei $\underline{\mathbf{Q}}$ eine orthogonale Matrix ist. In der Tat geht mit $\underline{\mathbf{Q}}=\underline{\mathbf{U V}}^{t r}$ und $\underline{\underline{\mathbf{P}}}=\underline{\underline{\mathbf{V W V}}}$ die Singluärwertzerlegung von $\underline{\underline{\mathbf{D}}}^{\Delta t}$ in die Polarzerlegung über. Damit besitzt die symmetrische positiv definite Matrix $\underline{\underline{\mathbf{P}}}$ als Eigenwerte die Singulärwerte $w^{i}$ und als Eigenvektoren die singulären Richtungen $\mathbf{v}^{i}$. Die Wirkung von $\underline{\mathbf{D} \Phi^{\Delta t}}$ kann als Streckung

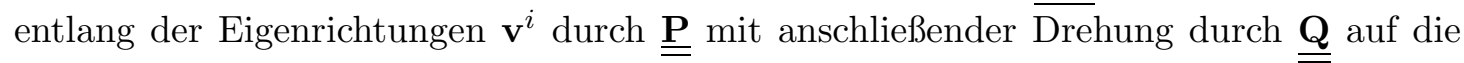
Richtungen $\mathbf{u}^{i}$ interpretiert werden. Wir wollen nun die Matrix $\underline{\underline{\mathbf{P}}}^{2}$ betrachten, welche die gleichen Eigenvektoren $\mathbf{v}^{i}$ wie $\underline{\underline{\mathbf{P}}}$ besitzt, aber die Eigenwerte $\left(w^{i}\right)^{2}$. Dazu multiplizieren wir Glg. (A.1) von rechts mit $\left(\underline{\underline{\mathbf{D}}}^{\Delta t}\right)^{t r}$ und vernachlässigen Terme der Ordnung $(\Delta t)^{2}$ :

$$
\begin{aligned}
\left(\underline{\underline{\mathbf{D} \Phi}}^{\Delta t}\right)^{t r} \underline{\underline{\mathbf{D} \Phi}}^{\Delta t} & =(\underline{\underline{\mathbf{I}}}+\Delta t \underline{\underline{J}})^{t r}(\underline{\underline{\mathbf{I}}}+\Delta t \underline{\underline{J}}) \\
& =\underline{\underline{\mathbf{I}}}+\Delta t\left(\underline{\underline{J}}^{t r}+\underline{\underline{J}}\right)+\mathcal{O}\left((\Delta t)^{2}\right) .
\end{aligned}
$$

${ }^{1}$ Die Idee zu den folgenden Ableitungen stammt von Dr. U. Dreßler, welche uns freundlicherweise einen bisher unveröffentlichtes Artikel zur Berechnung von lokalen Stabilitätsmultiplikatoren in zeitkontinuierlichen Systemen zur Verfügung gestellt hat. 


\section{A. Ableitung der lokal dynamischen Kopplung bei ODEs}

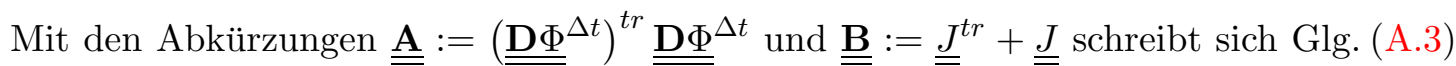
nun

$$
\underline{\underline{\mathbf{A}}}=\underline{\underline{\mathbf{I}}}+\Delta t \underline{\underline{\mathbf{B}}}
$$

wobei $\underline{\underline{\mathbf{A}}}=\underline{\underline{\mathbf{A}}}^{t r}$ und $\underline{\underline{\mathbf{B}}}=\underline{\underline{\mathbf{B}}}^{t r}$ symmetrische Matrizen sind. Aufgrund Glg. (A.4) besitzen $\underline{\underline{\mathbf{A}}}$ und $\underline{\mathbf{B}}$ die gleichen Eigenvektoren $\mathbf{v}^{i}=\mathbf{v}_{A}^{i}=\mathbf{v}_{B}^{i}$ und die reellen Eigenwerte sind durch

$$
\mu_{A}^{i}=1+\Delta t \mu_{B}^{i}
$$

miteinander verknüpft. Die Singulärwerte von $\underline{\mathbf{D} \Phi}^{\Delta t}$ lassen sich deswegen aus den Eigenwerten von $\underline{\underline{\mathbf{A}}}$ bzw. $\underline{\mathbf{B}}$

$$
w^{i}(\mathbf{x}, \Delta t)=\sqrt{\mu_{A}^{i}}=\sqrt{1+\Delta t \mu_{B}^{i}}
$$

bestimmen. Wie in Abschn. 3.2.5 diskutiert, stehen die Singluärwerte $w^{i}$ von $\mathbf{D} \Phi^{\Delta t}$ mit den Expansionsraten im Vektorfeld durch $\lambda_{\Delta t}^{i}=\ln \left(\left\|\underline{\underline{\mathbf{D}}}^{\Delta t} \mathbf{v}^{i}\right\|\right)=\ln \left(w^{i}\right) / \Delta t$ in Beziehung, wobei der Grenzwert von $\Delta t \rightarrow 0$ die lokalen Dissipationsstärken $\varepsilon^{i}$ der dynamischen Kopplung entgegen der Expansionsrichtung $\mathbf{v}^{i}$ im Geschwindigkeitsfeld $\mathbf{f}$ bestimmt.

$$
\begin{aligned}
\varepsilon^{i} & =\lim _{\Delta t \rightarrow 0} \lambda_{\Delta t}^{i}=\lim _{\Delta t \rightarrow 0} \frac{\ln \left(w^{i}(\mathbf{x}, \Delta t)\right)}{\Delta t} \\
& =\lim _{\Delta t \rightarrow 0} \frac{\sqrt{\mu_{A}^{i}}}{\Delta t}=\lim _{\Delta t \rightarrow 0} \frac{1+\Delta t \mu_{B}^{i}}{2 \Delta t} \\
& =\frac{\mu_{B}}{2}
\end{aligned}
$$

In der letzten Zeile wurde $\ln (1+x) \approx x$ für $|x| \ll 1$ benutzt, welches identisch für $\Delta t \rightarrow 0$ erfüllt ist. Die singulären Vektoren werden für $\Delta t \rightarrow 0$ identisch $\mathbf{u}=\mathbf{v}$, weil mangels Zeitentwicklung $(\Delta t=0)$ die Instabilität zwar weiter vorhanden ist, aber nicht mehr gedreht werden muß. Die dynamische Kopplung für ODEs ist dadurch ohne Berechnung bzw. Approximation des Flußes durch die Jacobimatrix des Geschwindigkeitsfeldes $\mathbf{f}$ bestimmt. Die expandierenden Richtungen $\mathbf{v}^{i}$ mit zugehörigen Dissipationsstärken $\varepsilon^{i}$ sind am Ort $\mathbf{x}$ eindeutig durch die Hauptachsentransformation von $\underline{\underline{\mathbf{B}}}=\underline{\underline{J}}^{\operatorname{tr}}(\mathbf{x})+\underline{\underline{J}}(\mathbf{x})$; bestimmt. 


\section{Literaturverzeichnis}

[1] H.D.I. Abarbanel, R.A. Katz, T. Galib, J. Cembrola, and T.W. Frison. Nonlinear analysis of high-reynolds-number flows over a buoyant axisymmetric body. Phys. Rev. E, 49:4003-4018, 1994. 75

[2] H.D.I. Abarbanel, N. Rulkov, and M. Sushchik. Generalized synchronization of chaos: The auxiliary system approach. Phys. Rev. E, 53(5):4528-4535, 1996. 16, 17,22

[3] V.S. Afraimovich, N.N. Verichev, and M.I. Rabinovich. Stochastic synchronization of oscillation in dissipative systems. Radiofizika, 29(9):1050-1060, 1986. 8, 16

[4] J.C. Alexander, J.C. Alexander, J.A. Yorke, and Z. You. Riddled basins. Intern. J. Bifurcation and Chaos, 2(4):795-813, 1992. 14

[5] J. Argyris, G. Faust, and M. Haase. Die Erforschung des Chaos. Vieweg, Braunschweig; Wiesbaden, 1994. 11, 70

[6] J. Arnhold, P. Grassberger, K. Lehnertz, and C.E. Elger. A robust method for detecting interdependences: application to intracranially recorded EEG. Physica D, 134:419-430, 1999. 18, 30

[7] P. Ashwin, J. Buescu, and I. Stewart. Bubbling of attractors and synchronization of chaotic oscillators. Phys. Lett. A, 193:127-139, 1994. 14

[8] E. Baake, M. Baake, H.G. Bock, and K.M. Briggs. Fitting ordinary differential equations to chaotic data. Phys. Rev. A, 45(8):5524 - 5529, 1992. 113

[9] G. Baier and S. Sahle. Design of hyperchaotic flows. Phys. Rev. E, 51(4):R2712R2714, 1995. 117, 117

[10] B. Blasius, A. Huppert, and L. Stone. Complex dynamics and phase synchronization in spatially extended ecological systems. Nature, 399:354-359, 1999. 1

[11] S. Boccaletti, J. Bragard, and F.T. Arecchi. Controlling and synchronizing space time chaos. Phys. Rev. E, 59(6):6574-6578, 1999. 30, 80, 80

[12] S. Boccaletti, J. Bragard, F.T. Arecchi, and H. Mancini. Synchronization in nonidentical extended systems. Phys. Rev. Lett., 83(3):536-539, 1999. 108, 109, 109, 110,110 


\section{Literaturverzeichnis}

[13] E. Bollt, Y. Lai, and C. Grebogi. Coding, channel capacity, and noise resistance in communicationg with chaos. Phys. Rev. Lett., 79(19):3787-3790, 1997. 112

[14] S. Bottani. Synchronization of integrate and fire oscillators with global coupling. Phys. Rev. E, 54(3):2334-2350, 1996. 2

[15] J.L. Breeden and A. Huebler. Reconstructing equations of motion from experimental data with unobserved variables. Phys. Rev. A, 42(10):5817-5826, 1990. 113

[16] R. Brown. Approximating the mapping between systems exhibiting generalized synchronization. Phys. Rev. Lett., 81(22):4835-4838, 1998. 16, 26

[17] R. Brown and L. Kocarev. A unifying definition of synchronization for dynamical systems, 2000. 2, 2

[18] R. Brown and N. Rulkov. Synchronization of chaotic systems: Transverse stability of trajectories in invariant manifolds. Chaos, 7(3):395-413, 1997. 11, 30, 80

[19] R. Brown, N. Rulkov, and N.B. Tufillaro. Synchronization of chaotic systems: The effects of additive noise and drift in the dynamics of the driving. Phys. Rev. E, 50(6):4488-4508, 1994. 15, 15

[20] R. Caponetto, L. Fortuna, G. Manganaro, and M. Xibilia. Synchronization-based nonlinear chaotic circuit identification. In SPIE, volume 2612, pages 48 - 56, 1995. 113

[21] M. Casdagli. Nonlinear prediction of chaotic time series. Physica D, 35:335 - 356, 1989. 113

[22] H. Chaté, A. Pikovsky, and O. Rudzick. Forcing oscillatory media: phase kinks vs. synchronization. Physica D, 131:17-30, 1999. 102

[23] A. Chernikov and G. Schmidt. Conditions for synchronization in Josephsonjunction arrays. Phys. Rev. E, 52(4):3415-3419, 1995. 3

[24] M.C. Cross and P.C. Hohenberg. Pattern formation outside of equilibrium. Rev. Mod. Phys., 65:851-1112, 1993. 82, 83, 83, 86, 92, 122

[25] M.E. Davies and K.M. Campbell. Linear recursive filters and nonlinear dynamics. Nonlinearity, 9:487-499, 1996. 16

[26] H. Dedieu, M.P. Kennedy, and M. Hasler. Chaos shift keying: Modulation and demodulation of a chaotic carrier using self-synchronizing chua's circuits. IEEE Trans. Circ. and Systems - II, 40(10):634-642, 1993. 112

[27] H. Dedieu and M. Ogorzalek. Identification and control of a particular class of chaotic systems. In SPIE, volume 2612, pages $148-156,1995$. 113, 114 


\section{Literaturverzeichnis}

[28] U. Dressler and G. Nitsche. Controlling chaos using time delay coordinates. Phys. Rev. Lett., 68(1):1-4, 1992. 73

[29] D.A. Egolf and H.S Greenside. Relation between fractal dimension and spatial correlation length for extensive chaos. Nature, 65(8):129, 1994. 86

[30] R.C. Elson, A. Selverston, R. Huerta, N. Rulkov, M. Rabinovich, and H. Abarbanel. Synchronous behaviour of two coupled biological neurons. Phys. Rev. Lett., 81(25):5692-5695, 1998. 3

[31] U. Ernst, K. Pawelzik, and T. Geisel. Synchronization induced by temporal delays in pulse-coupled oscillators. Phys. Rev. Lett., 74(9):1570-1573, 1995. 3, 3, 29

[32] H. Fujisaka and T. Yamada. Stability theory of synchronized motion in coupledoscillator systems. Prog. Theor. Phys., 69(1):32-47, 1983. 3, 3, 8, 13, 31, 80

[33] D. Gauthier and J. Bienfang. Intermittent loss of synchronization in coupled chaotic oscillators: Toward a new criterion for high-quality synchronization. Phys. Rev. Lett., 77(9):1751-1754, 1996. 11, 66, 80, 88

[34] L. Glass and M.C. Mackey. From Clocks to Chaos. Univ. Press, Princeton, USA, 1988. 2

[35] J. Goodwin, R. Brown, and L. Junge. The effect of noise on parameter estimation in systems with complicated dynamics. xxx.lanl.gov/abs/chao-dyn/9811002, 1999. 121

[36] C.M. Gray, P. König, A.K. Engel, and W. Singer. Oscillatory responses in cat visual cortex exhibit inter-columnar synchronization which reflects global stimulus properties. Ann. Rev. Physiol., 55:349-374, 1993. 3, 3

[37] R.O. Grigoriev. Control of spatially extended chaotic systems. In H.-G. Schuster, editor, Handbook of Chaos Control. Wiley-VCH, Weinheim,Germany, 1998. 79, 79, 101

[38] R.O. Grigoriev, M.C. Cross, and H.G. Schuster. Pinning control of spatiotemporal chaos. Phys. Rev. Lett., 79(5):2795-2798, 1997. 78, 79, 86

[39] K.S. Halle, C.W. Wu, M. Itoh, and L.O. Chua. Spread spectrum communication through modulation of chaos. Int. J. Bif. and Chaos, 3(2):469-477, 1993. 35, 112

[40] M. Hasler, Y. Maistrenko, and O. Popovych. Simple example of partial synchronization of chaotic systems. Phys. Rev. E, 58(5):6843-6846, 1998. 10

[41] G Hu, J. Xiao, L.O. Chua, and L. Pivka. Controlling spiral waves in a model of two-dimensional arrays of chua's circuits. Phys. Rev. Lett., 80(9):1884-1887, 1998. 30 


\section{Literaturverzeichnis}

[42] Gang Hu and Zhilin Qu. Controlling spatiotemporal chaos in coupled map lattice systems. Phys. Rev. Lett., 72(1):68-71, 1994. 30, 77, 78, 79

[43] N.E. Huang, Z. Shen, S.R. Long, M.L. Wu, H.H. Shih, Q. Zheng, N.C. Yen, C.C. Tung, and H.H. Liu. The empirical mode decomposition and the Hilbert spectrum for nonlinear and nonstationary time series analysis. Proc. R. Soc. London, A(454):903-995, 1998. 27

[44] B. Hübinger, R. Doerner, W. Martienssen, M. Herdering, R. Pitka, and U. Dressler. Controlling chaos experimentally in systems exhibiting large effective Lyapunov exponents. Phys. Rev. E, 50(2):932-948, 1994. 73

[45] H. Huijberts, T. Lilge, and H. Nijmeijer. Control perspective on synchronization and the Takens-Aeyels-Sauer reconstruction theorem. Phys. Rev. E, 59(4):46914694, 1999. 37, 37

[46] B. Hunt and E. Ott. Optimal periodic orbits of chaotic systems. Phys. Rev. Lett., 76(13):2254-2257, 1996. 10, 11, 11, 38

[47] B. Hunt, E. Ott, and J. Yorke. Differentiable generalized synchronization of chaos. Phys. Rev. E, 55(4):4029-4034, 1997. 24, 25

[48] Chriistiaan Huygens. Die Pendeluhr: Horologium oscillatorium (1763), volume 192 of Ostwald's Klassiker der exakten Wissenschaften. A. Heckscher und A. von Öttingen, Leipzig, 1913. 1

[49] J.M. Hyman and B. Nicolaenko. The Kuramoto-Sivashinski equation: a bridge between PDE's and dynamical systems. Physica D, 18:113-126, 1986. 122

[50] Y. Jiang and P. Parmananda. Synchronization of spatiotemporal chaos in asymmetrically coupled map lattices. Phys. Rev. E, 57(4):4135-4139, 1998. 30, 79

[51] G. Johnson, D. Mar, T. Carroll, and L. Pecora. Synchronization and imposed bifurcations in the presence of large parameter mismatch. Phys. Rev. Lett., 80(18):39563959, 1998. 37

[52] K. Josic. Invariant manifolds and synchronization of coupled dynamical systems. Phys. Rev. Lett., 80(14):3053-3056, 1998. 44, 108, 111

[53] L. Junge. Synchronisation nichtlinearer dynamischer Systeme. Diplomarbeit, Universität Göttingen, Februar 1997. 32

[54] L. Junge and U. Parlitz. Synchronization and control of spatially extended systems. In Proc. Int. Symposium on Nonlinear Theory and its Applications, NOLTA 98, Crans Montana, volume 1, pages 303-306, 1998. 81

[55] L. Junge and U. Parlitz. Phase synchronization of coupled Ginzburg-Landau equations. Phys. Rev. E, 62(1):438-441, 2000. 29 


\section{Literaturverzeichnis}

[56] L. Junge and U. Parlitz. Synchronization and control of coupled Ginzburg-Landau equations using local coupling. Phys. Rev. E, 61(4):3736-3742, 2000. 81

[57] L. Junge, U. Parlitz, Z. Tasev, and L. Kocarev. Synchronization and control of spatially extended systems using sensor coupling. Int. J. Bif. Chaos, 9(12):22652270, 1999. $30,81,81$

[58] L. Keefe. Properties of Ginzburg-Landau attractors associated with their Lyapunov spectra. Phys. Lett. A, 140(6'):317-322, 1989. 92, 95

[59] M.P. Kennedy and G. Kolumban. Digital communications using chaos. In G. Chen, editor, Controlling Chaos and Bifurcations in Engineering Systems, pages 477-500. CRC Press Inc., Boca Raton FL, 2000. 112

[60] L. Kocarev, P. Parlitz, and R. Brown. Robust synchronization of chaotic systems. Phys. Rev. E, 61(4):3716-3720, 2000. 24, 25, 25

[61] L. Kocarev and U. Parlitz. Generalized synchronization, predictability, and equivalence of unidirectionelly coupled dynamical systems. Phys. Rev. Lett., 76(11):18161819, 1996. 16, 17, 108

[62] L. Kocarev and U. Parlitz. Synchronizing spatiotemporal chaos in coupled nonlinear oscillators. Phys. Rev. Lett., 77(11):2206-2209, 1996. 30, 78, 79

[63] L. Kocarev, U. Parlitz, and B. Hu. Lie derivatives and dynamical systems. Chaos, Solitons \& Fractals, 9(8):1359-1366, 1998. 37

[64] L. Kocarev, Z. Tasev, and U. Parlitz. Synchronizing spatiotemporal chaos of partial differential equations. Phys. Rev. Lett., 79(1):51-54, 1997. 30, 80, 80, 86

[65] L. Kocarev, Z. Tasev, T. Stojanovski, and U. Parlitz. Synchronizing spatiotemporal chaos. Chaos, 7(4):635-643, 1997. 78, 79, 79, 86

[66] M. Koecher. Lineare Algebra und analytische Geometrie. Grundwissen Mathematik 2. Springer-Verlag, Berlin, 1989. 43

[67] Y. Lai and C. Grebogi. Synchronization of spatiotemporal chaotic systems by feedback control. Phys. Rev. E, 50(3):1894-1899, 1994. 79

[68] P.S. Landa. Nonlinear Oscillations and Waves in Dynamical Systems. Kluwer Academic Publishers, Dordrecht-Boston-London, 1996. 29

[69] D.G. Luenberger, editor. Introduction to dynamic systems, theory, models and applications. Wiley-VCH, New York, 1979. 36, 36

[70] P. Manneville. Liapunov exponents for the Kuramoto-Sivashinski model. In Lecture Notes in Physics, volume 230, pages 319-326. Springer Verlag,New York, 1985. 83, 84,84 


\section{Literaturverzeichnis}

[71] M. Markus, S.C. Müller, and G. Nicolis, editors. From Chemical to Biological Organization, volume 39 of Springer Series in Synergetics. Springer-Verlag, Berlin / Heidelberg / New York / London / Paris / Tokyo, 1988. 75, 80

[72] Th. Meyer, M.J. Bünner, A. Kittel, and J. Parisi. Hyperchaos in the generalized Rössler system. Phys. Rev. E, 56(5):5069-5082, 1997. 117

[73] A. Mielke. The complex Ginzburg Landau equation on large and unbounded domains: sharper bounds and attractors. Nonlinearity, 10(1):199-222, 1997. 94

[74] J. Milnor. On the concept of an attractor. Comm. Math. Phys., 99:177-195, 1985. 13

[75] R. Montagne, E. Hernandez-Garcia, and M. San Miguel. Winding number instability in the phase-turbulence regime of the complex Ginzburg-Landau equation. Phys. Rev. Lett., 77(2):267-270, 1996. 92

[76] Ö. Morgül and E. Solak. Observer based synchronization of chaotic systems. Phys. Rev. E, 54(5):4803-4811, 1996. 36

[77] Z. Néda, E. Ravasz, Y. Brechet, T. Vicsek, and A.-L. Barabási. The sound of many hands clapping. Nature, 304:849p, 2000. 2

[78] H. Nijmeijer and I. Mareels. An observer looks at synchronization. Transactions on Circuits and Systems, 44(10):882-890, 1997. 36, 36

[79] G. Osipov, A. Pikovsky, M.l Rosenblum, and J. Kurths. Phase synchronization effects in a lattice of nonidentical Rössler oscillators. Phys. Rev. E, 55(3):23532361, 1997. 28, 29

[80] E. Ott, C. Grebogi, and J.A. Yorke. Controlling chaos. Phys. Rev. Lett., 64(11):1196-1199, 1990. 73

[81] E. Ott and J.C. Sommerer. Blowout bifurcations: the occurrence of riddled basins and on-off intermitency. Phys. Lett. A, 188:39-47, 1994. 14, 14

[82] E. Otts. Chaos in Dynamical Systems. Cambridge Univ. Press, Cambridge, USA, 1993. 21,21

[83] M. Palusz, J. Kurths, U. Schwarz, D. Novotna, and I. Charvatova. Phase synchronization of sunspot numbers with the solar inertial motion. accepted for publication in Int. J. Bif. Chaos, 2000. 29, 105

[84] P. Panter. Modulation, noise and spectral analysis. Mcgraw-Hill, New York, 1965. 27

[85] N. Parekh, S. Parthasarathy, and S. Sinha. Global and local control of spatiotemporal chaos in coupled map lattices. Phys. Rev. Lett., 81(7):pp.1401-1404, 1998. 79 


\section{Literaturverzeichnis}

[86] U. Parlitz. Estimating model parameters from time series by autosynchronization. Phys. Rev. Lett., 76(8):1232-1235, 1996. 114

[87] U. Parlitz, L.O. Chua, L. Kocarev, K.S. Halle, and A. Shang. Transmission of digital signals by chaotic synchronization. Int. J. Bifurcation and Chaos, 2(4):973 - 977, 1992. 35, 112, 112

[88] U. Parlitz, L. Junge, and L Kocarev. Synchronization-based parameter estimation from time series. Phys. Rev. E, 54(6):6253-6260, 1996. 113, 114, 114, 114, 116, 116

[89] U. Parlitz, L. Junge, and L. Kocarev. Subharmonic entrainment of unstable period orbits and generalized synchronization. Phys. Rev. Lett., 79(17):3158-3161, 1997. 19,20

[90] U. Parlitz, L. Junge, and L. Kocarev. Chaos synchronization. In H. Nijmeijer and T.I. Fossen, editor, New Directions in Nonlinear Observer Design, Lecture Notes in Control and Information Sciences, pages 511-525. Springer-Verlag, Berlin / Heidelberg / New York / London / Paris / Tokyo, 244 edition, 1999. 25, 81

[91] U. Parlitz, L. Junge, and L. Kocarev. Nonidentical synchronization of identical systems. Int. J. Bif. Chaos, 9(12):2305-2309, 1999. 16

[92] U. Parlitz, L. Junge, W. Lauterborn, and L. Kocarev. Experimental observation of phase synchronization. Phys. Rev. E, 54(2):2115-2117, 1996. 28, 29

[93] U. Parlitz and L. Kocarev. General approach for chaotic synchronization with applications to communication. Phys. Rev. Lett., 74(25):5028-5031, 1995. 8, 30, $32,34,34,80$

[94] U. Parlitz and L. Kocarev. Multichannel communication using autosynchronization. Int. J. Bif. Chaos, 6(3):581-588, 1996. 35

[95] U. Parlitz and L. Kocarev. Synchronization of chaotic systems. In H.-G. Schuster, editor, Handbook of Chaos Control. Wiley-VCH, Weinheim,Germany, 1998. 81

[96] U. Parlitz, L. Kocarev, T. Stojanovski, and L. Junge. Chaos synchronization using sporadic driving. Physica D, 109:139-152, 1997. 8, 35, 35, 80, 114

[97] U. Parlitz, L. Kocarev, T. Stojanovski, and H. Preckel. Encoding messages using chaotic synchronization. Phys. Rev. E, 53(5):4351-4361, 1996. 8, 32, 35, 112

[98] U. Parlitz and I. Wedekind. Phase synchronization with binary coupling. accepted for publication in Int. J. Bif. Chaos, 2000. 29

[99] L. Pecora and T. Carroll. Synchronization in chaotic systems. Phys. Rev. Lett., 64(8):821 - 824, 1990. 3, 8, 30, 31, 80, 112 


\section{Literaturverzeichnis}

[100] L. Pecora, T. Carroll, and J. Heagy. Riddled bassins and other practical problems in coupled synchronized circuits. In SPIE, volume 2612, pages 25-36, 1995. 14

[101] A. Pentek, J.B. Kadtke, and Z. Toroczkai. Stabilizing chaotic vortex trajectories: an example of high-dimensional control. Phys. Lett. A, 224(1-2):85-92, 1996. 75

[102] K. Peters. Untersuchungen zur Dynamik gekoppelter chaotischer Systeme. Diplomarbeit, Universität Göttingen, Mai 2000. 77

[103] V. Petrov, E. Mihaliuk, S.K. Scott, and K. Showalter. Stabilizing and characterizing unstable states in high-dimensional systems from time series. Int. J. Bif. Chaos, 51(5):3988-3996, 1995. 75

[104] A. Pikovski, M. Zaks, M. Rosenblum, G. Osipov, and J. Kurths. Phase synchronization of chaotic oscillations in terms of periodic orbits. Chaos, 7(4):680-687, 1997. 29

[105] A. Pogromsky and H. Nijmeijer. Observer based robust synchronization of dynamical systems. Int. J. Bif. Chaos, 8(11):2243-2254, 1998. 36, 36

[106] W. Press, S. Teukolsky, W. Vetterling, and B. Flannery. Numerical Recipes in C. Cambridge University Press, 1994. 94, 116, 116

[107] K. Pyragas. Continuous control of chaos by self-controlling feedback. Phys. Lett. A, 170(6):421-428, 1992. 31, 32

[108] K. Pyragas. Weak and strong synchronization of chaos. Phys. Rev. E, 54(5):45084511, 1996. 24, 25

[109] T. Ritz, A. Schenck zu Schweinsberg, U. Dressler, R. Doerner, B. Hübinger, and W. Martienssen. Chaos control with adjustable control times. Chaos, Solitons 8 Fractals, 8(9):1559-1576, 1997. 73

[110] M. Rosenblum, A. Pikovsky, and J. Kurths. Phase synchronization of chaotic oscillators. Phys. Rev. Lett., 76(11):1804-1807, 1996. 28, 29, 103

[111] M. Rosenblum, A. Pikovsky, and J. Kurths. From phase to lag synchronization in coupled chaotic oscillators. Phys. Rev. Lett., 78(22):4193-4196, 1997. 28

[112] O.E. Rössler. An equation for continuous chaos. Phys. Lett. A, 57:397-398, 1976. 11

[113] R. Roy and K.S. Thornburg. Experimental synchronization of chaotic lasers. Phys. Rev. Lett., 72(13):2009-2012, 1994. 3

[114] N. Rulkov, M. Sushchik, L. Tsimring, and H. Abarbanel. Generalized synchronization of chaos in directionally coupled chaotic systems. Phys. Rev. E, 51(2):980-994, 1995. 16, 18, 26, 108 


\section{Literaturverzeichnis}

[115] Nikolai F. Rulkov. Images of synchronized chaos: Experiments with circuits. Chaos, 6(3):262-279, 1996 . 8

[116] N.V. Rulkov, A.R. Volkovskii, A. Rodriguez-Lozano, E. Del Rio, and M.G. Velgarde. Mutual synchronization of chaotic self-oscillators with dissipative coupling. Int. J. Bif. Chaos, 2(3):669-676, 1992. 30

[117] T. Sauer, J.A. Yorke, and M. Casdagli. Embedology. J. Stat. Phys., 65(3,4):579 616, 1991. 36

[118] C. Schäfer, M. Rosenblum, J. Kurths, and H.-H. Abel. Heartbeat synchronized with ventilation. Nature, 392(6673):239-240, 1998. 3, 29, 105

[119] B. Schechter. How the brain gets rhythm. Science, 274(5286):339, 1996. 3

[120] S.J. Schiff, P. So, T. Chang, R.E. Burke, and T. Sauer. Detecting dynamical interdependence and generalized synchrony through mutual prediction in a neural ensemble. Phys. Rev. E, 54(6):6708-6724, 1996. 18

[121] T. Shibata and K. Kaneko. Collective chaos. Phys. Rev. Lett., 81(19):4116-4119, 1998. 76,76

[122] K.M. Short. Steps toward unmasking secure communications. Int. J. Bif. Chaos, 4(4):959-977, 1994. 112

[123] W. Singer. Synchronization of cortical activity and its putative role in information processing and learning. Nature, 338:334, 1989. 3, 3, 30

[124] T. Stojanovski, L. Kocarev, and U. Parlitz. Driving and synchronizing by chaotic impulses. Phys. Rev. E, 54(2):2129-2131, 1996. 35

[125] T. Stojanovski, L. Kocarev, Ulrich Parlitz, and R. Harris. Sporadic driving of dynamical systems. Phys. Rev. E, 55(4):4035-4048, 1997. 35

[126] T. Stojanovski, U. Parlitz, L. Kocarev, and R. Harris. Exploiting delay reconstruction for chaos synchronization. Phys. Lett. A, 233:355-360, 1997. 36, 68, 115

[127] M. Sushchik. Synchronized chaotic oscillations. PhD thesis, University of California, San Diego, 1996. 80

[128] F. Takens. Detecting strange attractors in turbulence. Lecture notes in Mathematics, 898:366-381, 1981. 36, 68

[129] Y. Takiguchi, H. Fujino, and J. Ohtsubo. Experimental synchronization of chaotic oscillations in externally injected semiconductor lasers in a low-frequnecy fluctuation regime. Opt. Lett., 24(22):1570-1572, 1999. 3 


\section{Literaturverzeichnis}

[130] Z. Tasev, L. Junge, L. Kocarev, and U. Parlitz. Synchronization of KuramotoSivashinski equations using spatially local coupling. Int. J. Bif. Chaos, 10(4):869873, 2000. $81,81,84,88$

[131] P. Tass, M. Rosenblum, J. Weule, J. Kurths, A. Pikovsky, J. Volkmann, A. Schnitzler, and H. Freund. Detection of n:m phase locking from noisy data: Application to magnetoencephalography. Phys. Rev. Lett., 81:3291-3294, 1998. 3, 29, 105

[132] F.E. Thau. Observing the state of non-linear dynamic systems. Int. J. Control, $17(3): 471-479,1973$. 36, 36

[133] B. van der Pol. Forced oscillations in a circuit with non-linear resistance. Phil. Mag., 7-3:65 - 80, 1927. 1

[134] B. van der Pol and J. van der Mark. Frequency demultiplication. Nature, 120:363 $-364,1926.27$

[135] S. Venkataramani, B. Hunt, and E. Ott. Bubbling transition. Phys. Rev. E, 54(2):1347-1360, 1996. 14

[136] H. Voss, M. Bünner, and M. Abel. Identification of continuous, spatiotemporal systems. Phys. Rev. E, 57(3):2820-2823, 1998. 113

[137] S. Wiggins. Normally Hyperbolic Invariant Manifolds in Dynamical Systems. Springer Verlag, New York, 1994. 25

[138] H.G. Winful and L. Rahmen. Synchronized chaos and spatiotemporal chaos in arrays of coupled lasers. Phys. Rev. Lett., 65(13):1575, 1990. 3, 75

[139] J.H. Xiao, G. Hu, and Q. Zhilin. Synchronization of spatiotemporal chaos and its application to multichannel spread-spectrum communication. Phys. Rev. E, $77(20): 4162-4165,1996.79$

[140] Y. Zhang, J. Kastrup, R. Klann, and K. Ploog. Synchronization and chaos by resonant tunneling in GaAs/AIAs superlattices. Phys. Rev. Lett., 77(14):30013004, 1996. 3

[141] C. Zhou and C.-H. Lai. Analysis of spurious synchronization with positive conditional Lyapunov exponents in computer simulations. Physica D, 135:1-23, 2000. 47,55

[142] S. Zoldi and H. Greenside. Spatially localized unstable periodic orbits of a highdimensional chaotic system. Phys. Rev. E, 57(3):R2511-R2514, 1998. 11 


\section{Danksagung}

Herzlich bedanken möchte ich mich bei PD Dr. Ulrich Parlitz, der diese Arbeit ermöglicht hat und stets mit Rat und Tat beiseite stand. Die Zusammenarbeit war immer von einer konstruktiven, offenen und besonders kreativen Atmosphäre geprägt, so daß niemals Druck aufkam und wenn, dann stets nur im positiven Sinne.

Für die fruchtbare wissenschaftliche Zusammenarbeit und viele interessante Diskussionen möchte ich mich bei Prof. Dr. Ljupco Kocarev, Dr. Zarko Tasev, Prof. Dr. H.D.I. Abarbanel, Dr. M. Sushchik und Prof. Dr. R. Brown herzlich bedanken.

Ich bedanke mich auch bei Prof. Dr. Werner Lauterborn für die hervorragende Infrastruktur und freundliche und aufgeschlossene Atmospäre in unserem Institut.

Allen Mitgliedern der Arbeitsgruppe Nichtlineare Dynamik bin ich sehr zu Dank verpflichtet für viele anregende und konstruktive Diskussionen, die zur Fertigstellung dieser Arbeit beigetragen haben, insbesondere Dipl. Phys. S. Luther, Dipl. Phys. J. Wichard, Dipl. Phys. C. Merkwirth, Dipl. Phys. M. Wiesenfeldt, Dipl. Phys. J. Bröcker und Dr. C.D. Ohl für wertvolle Hilfen bei Problemen verschiedenster Art.

Auch unserer Fotografin, den Angestellten im Büro, den Werkstätten und unseren Softund Hardwarespezialistenmöchte ich für die freundliche Hilfe bei den vielen kleinen und großen Problemen danken.

Bei allen Institutsangehörigen möchte ich mich für die freundliche und offene Atmosphäre bedanken.

Besonderer Dank gilt auch meinen Eltern Irene und Heinz Junge und meinen Bruder Berndt, für das Zusammenstehen unserer Familie in vielen schwierigen Phasen, und daß man sich auf sie jederzeit und bei allen Problemen hundertprozentig verlassen kann.

Aus den gleichen Gründen danke ich meiner Freundin Andrea und zusätzlich noch für die Rücksichtnahme während der Anfertigung dieser Arbeit. 